\title{
Reduction of Power Supply Induced Jitter with Applications to DDR Controllers
}

\author{
By \\ Xinjie Wang
}

A thesis submitted to the Faculty of Graduate and Postdoctoral Affairs in partial fulfillment of the requirements for the degree of

Doctor of Philosophy

in

Electrical Engineering

Ottawa-Carleton Institute for Electrical and Computer Engineering

Department of Electronics

Carleton University

(C)2016

Xinjie Wang 


\begin{abstract}
It is desirable to improve the tradeoff between the power supply induced jitter (PSIJ) performance and the cost for the clock distribution circuit in a double data rate (DDR) controller. This thesis proposes the following techniques to achieve the goal:

The static phase offset (SPO) is the dominant mechanism causing reference spurs in the spectrum of the multiplying delay-locked loop (MDLL) output. With a high-gain stage inserted between the phase detector/phase frequency detector and the charge pump, the equivalent SPO has been decreased by a factor equal to the gain of the gain stage. The effectiveness of the proposed technique has been verified at both behavioral level with a Simulink model and the transistor level with circuit simulation results.
\end{abstract}

This thesis also presents a very simple and highly accurate expression for the power supply-induced jitter sensitivity transfer function for CMOS buffer chains. The transfer function is mainly a function of the maximum and minimum propagation delay of the buffer chain.

Lastly in this thesis, a concise method is proposed for converting the frequency domain equivalent serial resistance (ESR) and equivalent serial capacitance (ESC) to an approximate broadband equivalent circuit which can be readily used in time domain jitter analysis.

Finally a case study demonstrates that, with the proposed techniques in this dissertation, the MDLL based clock distribution circuit for a DDR controller improves the tradeoff 
between performance and cost compared to the traditional phase locked loop (PLL) based clock distribution circuit. 


\section{Acknowledgement}

I would like to express my deep appreciation and gratitude to my supervisor, Dr. Leonard MacEachern, for his guidance, knowledge and critical suggestions, which are effective and efficient.

I would like to express my deep appreciation and gratitude to my supervisor, Dr. Tad Kwasniewski, for his guidance, knowledge, support, and great contribution.

I also wish to extend my appreciation and gratitude to Dr. Yuming Tao, Dr. Steven McGArry, Dr. Samy Mahmoud, Dr. Voicu Groza, Dr. James Opp and Dr. Maitham Shams for their time and valuable inputs.

Thanks to my wife Lin and my daughter Kate for their patience, love and great sacrifice. Thanks to my parents for their constant support and encouragement. 


\section{Table of Contents}

Abstract

Chapter 1 Introduction .......................................................................................................................... 1

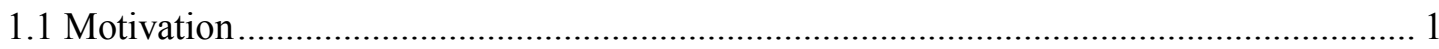

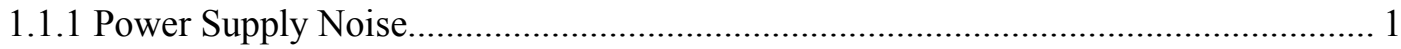

1.1.2 DDR Controller and Design Challenges............................................................. 2

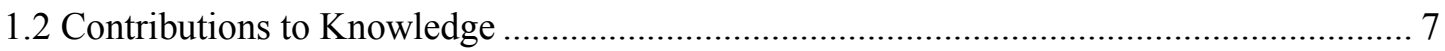

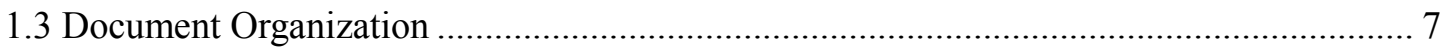

Chapter 2 Overview of DLL-based Frequency Multipliers.....................................................9

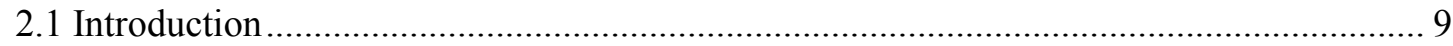

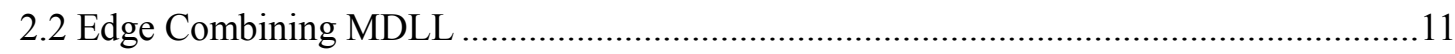

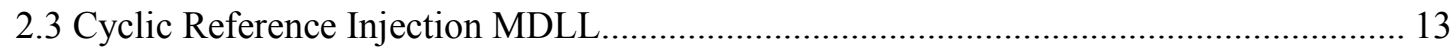

2.4 Overview of Reported Spur Reduction Techniques for Cyclic Reference Injection MDLL

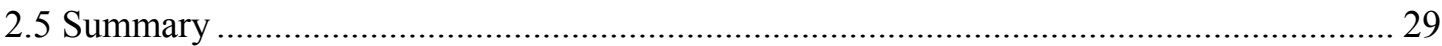

Chapter 3 Proposed Low-spur Reference Injection MDLL ................................................. 32

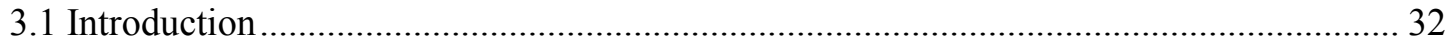

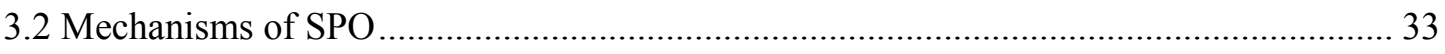

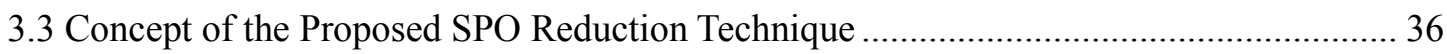

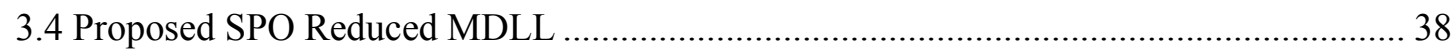

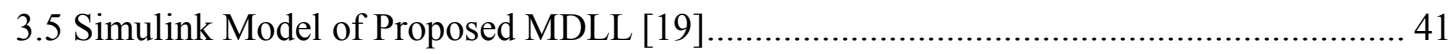

3.6 Simulation of the Proposed MDLL at the Transistor Level ............................................ 46

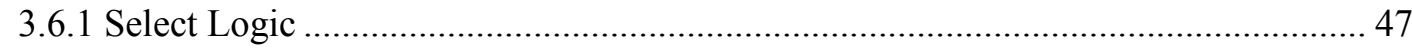

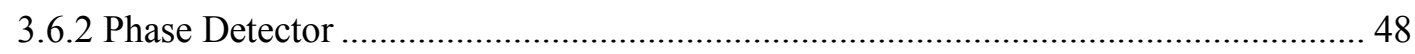

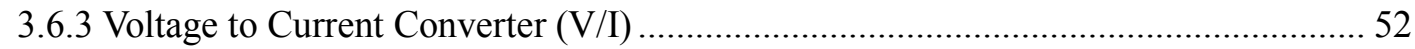

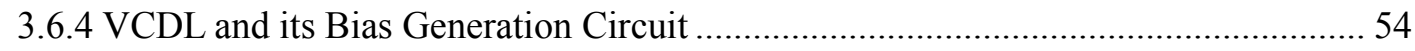

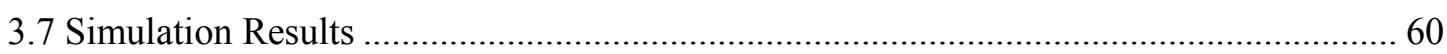

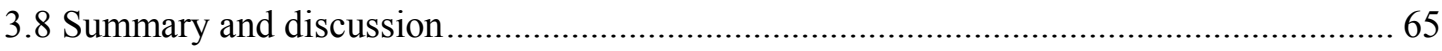

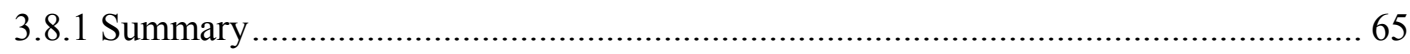

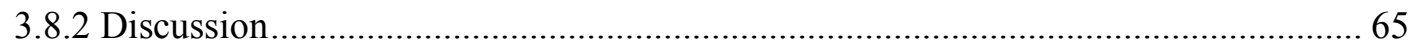

Chapter 4 Power Supply Induced Jitter Sensitivity of a Buffer Chain .................................... 67

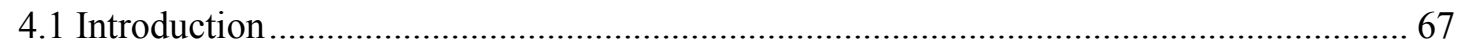

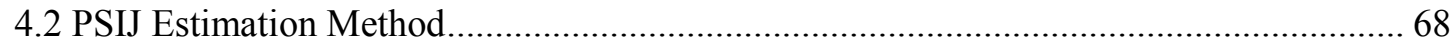

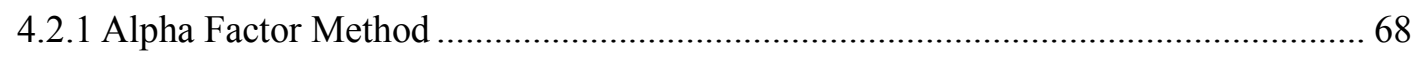

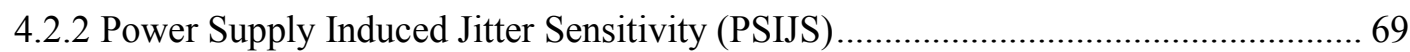




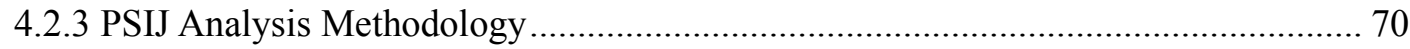

4.3 Overview of Power Supply Induced Jitter Sensitivity of a Buffer Chain ........................ 72

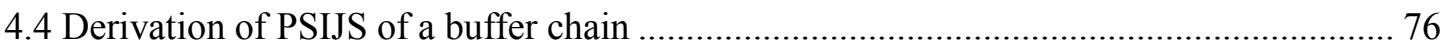

4.4.1 Equivalent RC Model of a Buffer Chain ................................................................. 77

4.4.2 Small Signal Equant Delay Gain Model of a Buffer Chain ..................................... 79

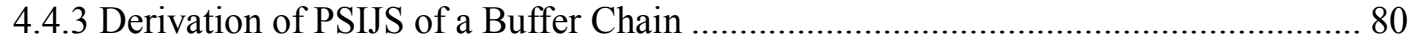

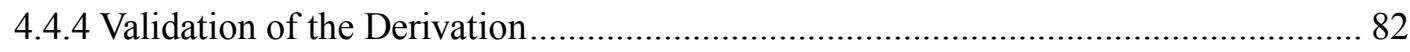

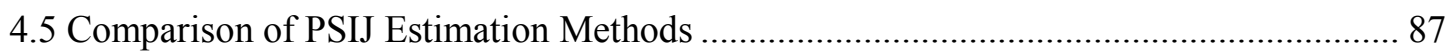

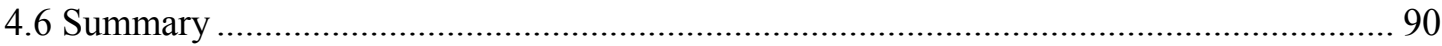

Chapter 5 Method of Extracting Rdie and Cdie...............................................................92

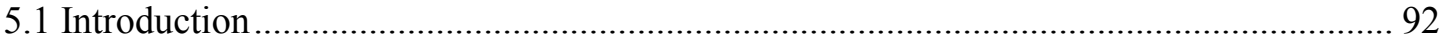

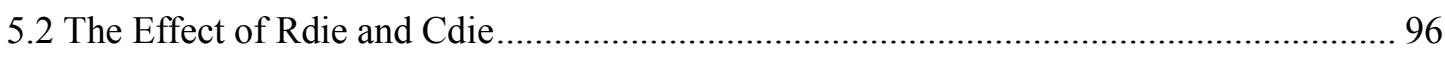

5.3 Parasitic Models for IC (integrated circuit) Layout Extraction....................................... 99

5.3.1 Layout Extraction Evaluation Areas ....................................................................... 99

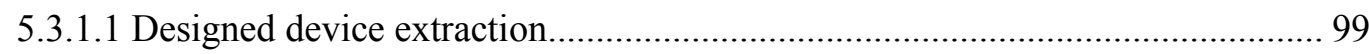

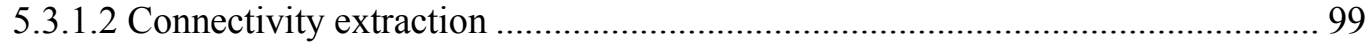

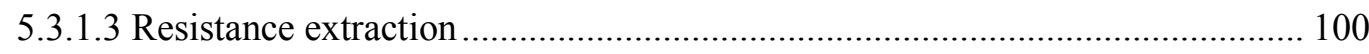

5.3.1.4 Capacitance and inductance estimation..................................................... 100

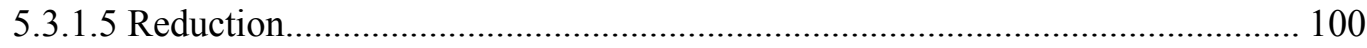

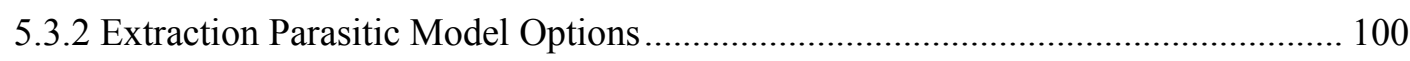

5.3.2.1 Lumped Capacitance (C-only) and lumped capacitance with coupled capacitors

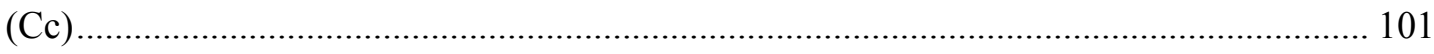

5.3.2.2 Distributed resistance only (R-only)........................................................... 102

5.3.2.3 Distributed resistance and capacitance (RC) and distributed resistance and

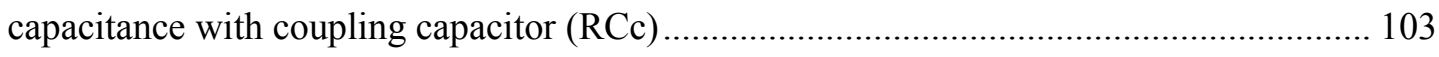

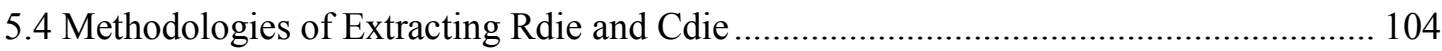

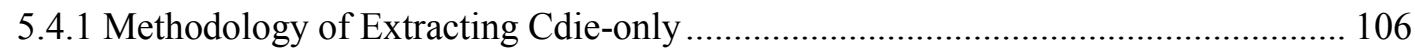

5.4.2 Methodologies of Extracting Both Rdie and Cdie................................................ 107

5.4.2.1 Method A: Unit voltage AC analysis method................................................. 108

5.4.2.2 Method B: Unit current AC analysis method .................................................. 109

5.5 Proposed Broadband Equivalent Circuit of Frequency Domain Rdie and Cdie ...............110

5.6 Validation of the Proposed Broadband Equivalent Circuit ............................................115

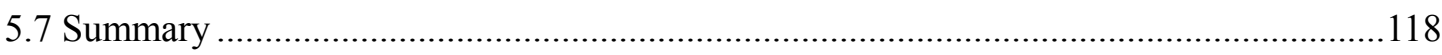

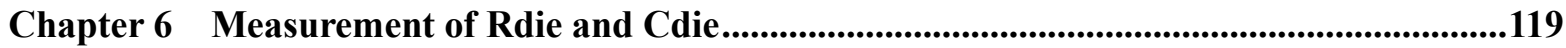

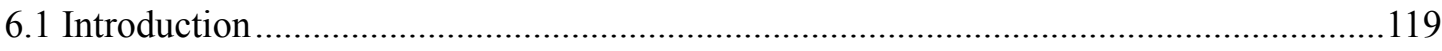

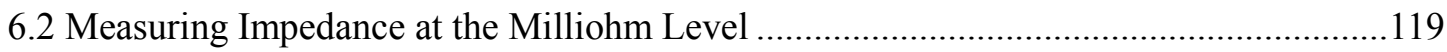

6.3 Rdie/Cdie Measurement of DDR Controller Test Chips............................................... 123

6.3.1 Extracting Decoupling Capacitance Cpkg and Inductance Lpkg on a Bare Package 
Substrate 127

6.3.2 Extracting Cdie and Lpkg on Packaged Devices ................................................. 129

6.3.3 Improved Extracted Series RLC Model vs. Original Measured S21 ...................... 134

6.3.4 Comparison between Simulated Results and Measured Results ............................ 142

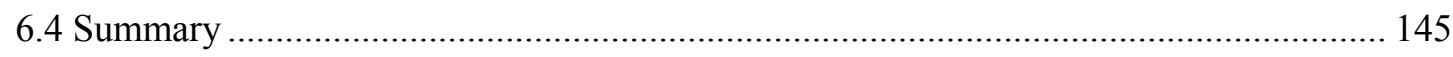

Chapter 7 A Case Study and Conclusion............................................................................................... 146

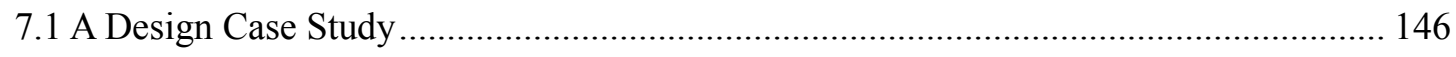

7.1.1 Sizing the DECAP to Satisfy the Supply Noise Specification................................. 148

7.1.1.1 Sizing the DECAP for the PLL based clock distribution circuit ...................... 148

7.1.1.2 Sizing the DECAP for the MDLL based clock distribution circuit .................. 149

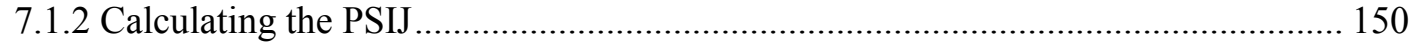

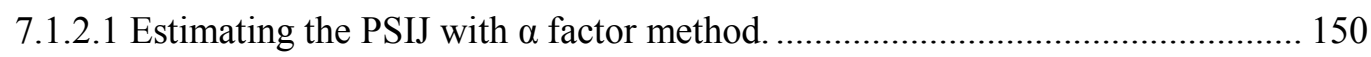

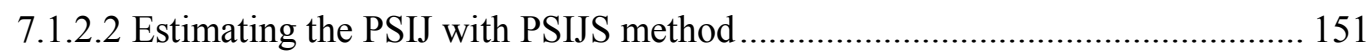

7.1.3 Performance Comparison between the Two Clock Distribution Circuits ................ 152

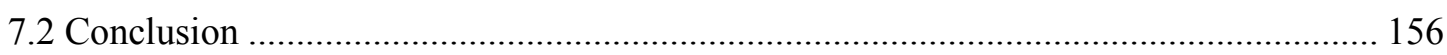

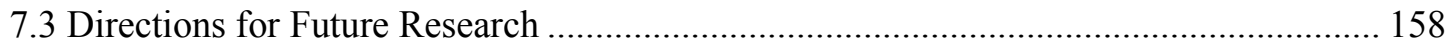

7.4 Publications that Arose from the Research ............................................................ 159 


\section{List of Abbreviations}

ADS

ASIC

BGA

Cdie

$\mathrm{CP}$

DAC

DDR

DLF

DLL

DTC

DUT

DXRO

DZ

ED

ESR

ESC

FFT

FLL

GRO

IFFT

LMS

LPDDR

$\mathrm{MC}$

MDLL

MUX

ODC

PD

PECL

PFD

PG

PLL

PDN

PSIJ

PSIJS

Rdie

SDRAM

SI
Advanced Design System

Application-Specific Integrated Circuit

Ball Grid Array

on-die power grid equivalent capacitance

Charge Pump

Digital to Analog Converter

Double Data Rate

Digital Loop Filter

Delay Locked Loop

Digital to Time Converter

Device Under Test

Digitally-controlled multipleXed Ring Oscillator

Dead Zone

Error Detector

Equivalent Serial Resistance

Equivalent Serial Capacitance

Fast Fourier Transform

Frequency-Locked Loop

Gated Ring Oscillator

Inverse Fast Fourier Transform

Least-Mean Square

low-power DDR

Modulus Control

Multiplying Delay-Locked Loops

Multiplexer

On-die Decoupling Capacitor

Phase Detector

Period Error Compensation Loop

Phase Frequency Detector

Pulse Generator

Phase Locked Loop

Power Distribution Network

Power Supply Induced Jitter

Power Supply Induced Jitter Sensitivity on-die power grid equivalent resistance

Synchronous Dynamic Random Access Memory

Signal Integrity 
SPO

SSN

SSO

TDC

$\mathrm{VCDL}$

$\mathrm{VCO}$

VNA
Static Phase Offset

Simultaneous Switching Noise

Simultaneous Switching Output

Time to Digital Converter

Voltage Controlled Delay Line

Voltage Controlled Oscillator

Vector Network Analyzer 


\section{List of Tables}

Table 2.1 Comparison of PLL and MDLL .............................................................................10

Table 2.2 Classification of DLL based frequency multipliers ................................................10

Table 2.3 Performance comparison of selected MDLLs.............................................................30

Table 3.1 Simulation results of the Simulink model of the MDLL ..............................................44

Table 3.2 Transistors and capacitors used in the circuit of PD...................................................52

Table 3.3 Transistors and capacitors used in the circuit of V/I..................................................54

Table 3.4 Transistors used in the VCDL and the bias generation circuit. ....................................60

Table 3.5 Deterministic jitter comparison with published works.............................................64

Table 4.1 Transistor sizes, supply voltages and input signals summary of all buffer chains............. 84

Table 4.2 Summary of the PSIJ obtained from different methods. ..............................................89

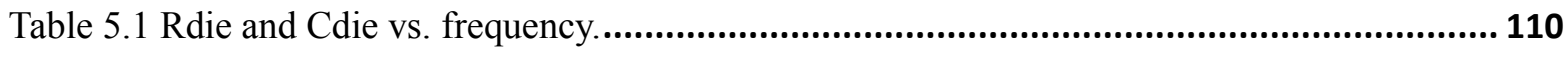

Table 6.1 Test devices for the measurement ........................................................................ 124

Table 6.2 Measured Cpkg and Lpkg of the flip chip substrate and the wire bonding chip substrate 128

Table 6.3 Extracted Cdie and Lpkg of packaged devices.......................................................... 133

Table 7.1 Summary of the simulation results from the clock distribution circuits with PLL/MDLL. 


\section{List of Figures}

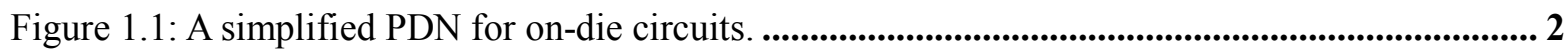

Figure 1.2: DDR interface block diagram. ............................................................................................... 3

Figure 2.1: Block diagram of edge MDLL and its typical waveform [45] (C) 2000 IEEE....................11

Figure 2.2: Block diagram of the MDLL with cyclic reference injection [43] (C) 2002 IEEE............. 15

Figure 2.3: Frequency multiplier timing [43] (C) 2002 IEEE.......................................................... 15

Figure 2.4: Block diagram of cyclic reference injection MDLL with period error compensation loop [13] (C) 2006 IEEE...................................................................................................... 18

Figure 2.5: The N-th fout cycle has a significant different period compared with the rest cycles of fout due to the static phase offset in the PFD. .......................................................................................... 18

Figure 2.6: CMOS implementation of the ED [13] (C) 2006 IEEE. ...................................................... 19

Figure 2.7: Operation of the ED [13] (C) 2006 IEEE.......................................................................................... 20

Figure 2.8: Block diagram of MDLL and tuning method leveraging a TDC and correlated double-sampling [15] (C) 2008 IEEE......................................................................................... 21

Figure 2.9: Gated ring oscillator time-to-digital converter [15] (C) 2008 IEEE. ..................................... 23

Figure 2.10: MDLL with proposed aperture tuning mechanism [14] (C) 2011 IEEE............................. 25

Figure 2.11: A fractional-N MDLL [16] (C) 2014 IEEE.............................................................................. 27

Figure 2.12: A low reference spur digital MDLL [42] (C) 2013 IEEE................................................. 29

Figure 3.1: PLL/MDLL output signal in locked states. (a) PLL/MDLL reference signal; (b) PLL output with positive SPO; (c) PLL output with negative SPO; (d) MDLL output with negative SPO; (e) MDLL output with positive SPO ..................................................... 33

Figure 3.2: The conceptual diagram of the charge pump and charge pump current with non-ideal effects in the locked state. (a) The charge pump diagram; (b) Charge pump output current due to the current leakage; (c) Charge pump output current due to the current mismatch [52].

Figure 3.3: Conceptual diagram of the proposed SPO reduction technique. (a) S-domain model of MDLL; (b) S-domain model of MDLL with SPO $A S P O$; (c) S-domain model of MDLL with SPO $\theta S P O$ and a gain stage insertion; (d) Equivalent SPO becoming $\theta S P O / G$ after a gain stage insertion [19]. ........................................................................................................................ 37

Figure 3.4: The time domain model of MDLL with the proposed SPO reduction technique. ............. 38

Figure 3.5: Block diagrams of (a) the proposed MDLL and (b) the PD........................................... 39 
Figure 3.6: Simplified timing diagram of the PD..................................................................................... 41

Figure 3.7: Simulink model of the proposed MDLL........................................................................................ 43

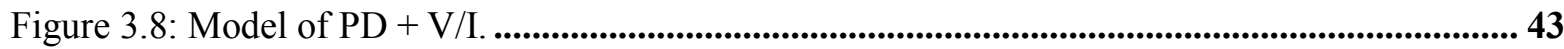

Figure 3.9: Spectral plots of VCDL output under different conditions. (a) SPO=0, Gain1=1; (b) $\mathrm{SPO}=2 \mathrm{e}-11 \mathrm{ps}$, Gain1=1; (c) SPO=2e-11ps, Gain1=10; (d) SPO=2e-11ps, Gain1=50..... 45

Figure 3.10: spur values vs the gain $(G)$ of the insertion gain stage...................................................... 46

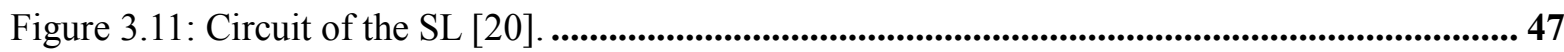

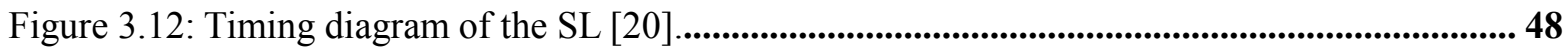

Figure 3.13: Schematic of the PD.............................................................................................................. 48

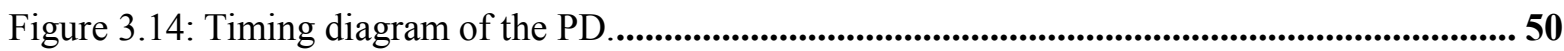

Figure 3.15: The PG circuit. .......................................................................................................................................5 50

Figure 3.16: The circuit of series M5 and CS and their equivalent RC charging circuit..................51

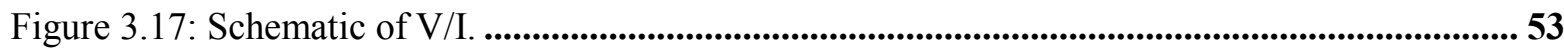

Figure 3.18: The VCDL and its bias generation circuit..........................................................................54

Figure 3.19: The schematic of MUX circuit................................................................................................55

Figure 3.20: Transmission gate. (a) schematic; (b) equivalent small signal RC model. ...................... 56

Figure 3.21: The delay element comprising an inverter and a transmission gate. (a) schematic; (b) equivalent RC model. ..............................................................................................57

Figure 3.22: Single inverter delay element. (a) schematic; (b) equivalent RC model.......................... 57

Figure 3.23: MDLL output $f o b$ and the converted voltage pulse-train signal $V$ int0. (a) In the initial locked state, the 12th cycle has a smaller period than the rest of the cycles; (b) In stable state, the 12 th cycle has a similar period to the rest of the cycles.......................................61

Figure 3.24: Spectrum of the proposed MDLL output...........................................................................63

Figure 3.25: Spectrum of the conventional MDLL output .............................................................6 63

Figure 3.26: Jitter sequence plots for MDLL and PLL with 60mV peak-to-peak sawtooth shaped

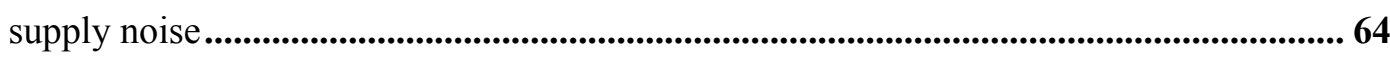

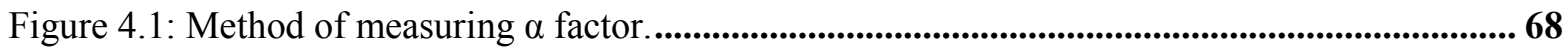

Figure 4.2: Methodology to predict power supply induced jitter. ......................................................... 70

Figure 4.3: Effect of power and ground voltage fluctuations on the propagation delay for the low-to-high output transition and the representation of each voltage waveform [26] (C)

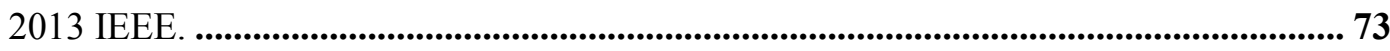


Figure 4.4: Transfer functions of supply voltage fluctuation to jitter obtained from HSPICE simulation and analytical expression [26] (C) 2013 IEEE.

Figure 4.5: Phase variation and jitter in the VCDL depending on the variation of the control voltage. The dashed line implies the jitter with $100 \mathrm{mVp}$-p of the variation of the control voltage. The solid line and the wavy line show the jitter with $75 \mathrm{mVp}$-p and $50 \mathrm{mVp}$-p of the control voltage, respectively [28] (C) 2014 IEEE. ..................................................................... 76

Figure 4.6: A buffer chain with a noisy power supply............................................................................ 77

Figure 4.7: Equivalent RC circuit model. (a) nMOS and pMOS transistor models; (b) An inverter

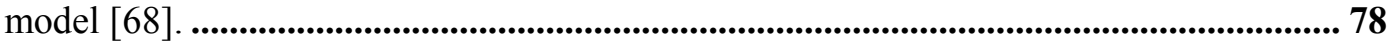

Figure 4.8: Buffer chain equivalent $N$ sections of small delay stages and the $i$-th section of delay stage.

Figure 4.9: Plots of PSIJS from Hspice simulation results and expression (4.18). (a) Buffer chain 1;

(b) Buffer chain 2; (c) Buffer chain 3; (d) Buffer chain 4.................................................... 85

Figure 4.10: Buffer chain power supply sawtooth noise signal........................................................88

Figure 4.11: PSIJ sequences calculated through PSIJS of (a) Hspice result; (b) theoretical result..... 88

Figure 4.12: Hspice simulation results of PSIJ of buffer chain 4............................................................ 90

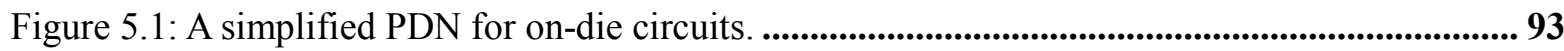

Figure 5.2: Cross section of a CMOS inverter and the physical locations of intrinsic ODC [71].......94

Figure 5.3: An inverter and its equivalent circuits. (a) Inverter; (b) Equivalent circuit of (a) as

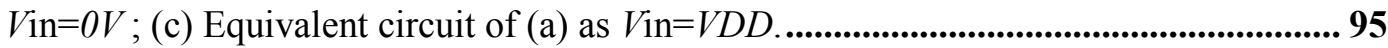

Figure 5.4: The entire extracted on-die power delivery network. ..........................................................96

Figure 5.5: Simplified PDN system with dead short board assumption.............................................97

Figure 5.6: PDN impedance vs package inductance. ................................................................................... 97

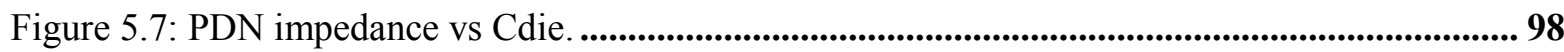

Figure 5.8: PDN impedance vs Rdie. ....................................................................................................... 98

Figure 5.9: A simplified layout of two instances with two nets. ............................................................. 101

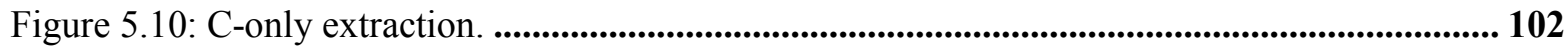

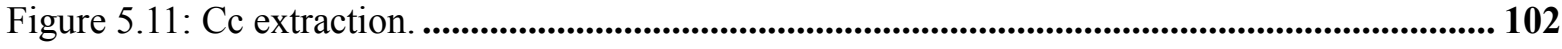

Figure 5.12: Net has been divided into $\mathrm{N}$ segments for parasitic resistance extraction. .................... 103

Figure 5.13: R-only extraction. ..................................................................................................................... 103

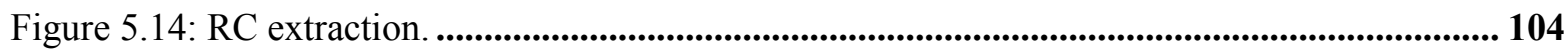


Figure 5.15: RCc extraction.

Figure 5.16: Cdie-only extraction method. (a) Testbench of Cdie-only extraction; (b) Ramp voltage added to power supply; and (c) Current flowing into node VDD due to the added ramp voltage shown in (b)

Figure 5.17: Method A: Unit voltage AC analysis method.

Figure 5.18: Method B: Unit current AC analysis method. 109

Figure 5.19: Parallel N series-RC-pairs circuit 111

Figure 5.20: Parallel N=1 series-RC-pairs circuit. 111

Figure 5.21: Parallel N=2 series-RC-pairs circuit. .112

Figure 5.22: The approximate broadband equivalent circuit of the frequency domain Rdie/Cdie in

Table 5.1

Figure 5.23: Comparison of the simulation results from extracted netlist and the approximate broadband equivalent circuit. (a) Rdie; (b) Cdie; (c) Impedance of series Rdie and Cdie.

Figure 5.24: Rdie, Cdie and total impedance $\mathrm{Z}$ error percentage of the approximate broadband equivalent circuit to the extracted results. (a) Rdie error percentage; (b) Cdie error percentage; (c) Total impedance error percentage.

Figure 6.1: A method of using a VNA 2-port for measurement of low impedance

Figure 6.2: Zconnection1 and Zconnection2 approximately absorbed by ZSRC and ZRCVR respectively

Figure 6.3: A RLC circuit.

Figure 6.4: The imaginary part of the impedance Zdut measured from the circuit shown in Figure 6.3.

Figure 6.5: Equivalent circuit looking into VDD/VSS................................................................................ 124

Figure 6.6: Top level measurement setup........................................................................................................ 125

Figure 6.7: The detailed equipment arrangement for the measurement setup..................................... 125

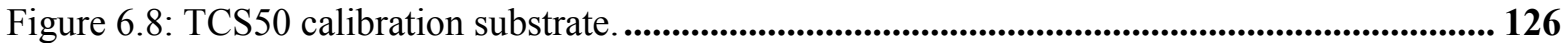

Figure 6.9: Probes on the bare substrate and packaged devices............................................................. 126

Figure 6.10: The imaginary parts of the bear substrates of the flip chip and the wire bond chip...... 128

Figure 6.11: Extracted ESR of substrates..................................................................................................... 129

Figure 6.12: Packaged devices PDN for (a) the wire bond and (b) the flip chip packing.................. 131 
Figure 6.13: The measured imaginary parts of Zdut of devices with the flip chip or the wire bond package........................................................................................................................................... 132

Figure 6.14: Extracted ESR of packaged devices...................................................................... 133

Figure 6.15: Extracted series RLC model of chipA. .................................................................................. 134

Figure 6.16: Original measured S21 of chipA vs. extracted RLC model S21.................................... 135

Figure 6.17: Improved extracted RLC model of chipA............................................................................... 136

Figure 6.18: Original measured S21 of chipA vs improved extracted RLC model S21.................... 137

Figure 6.19: Improved extracted series RLC model of chipB................................................................ 138

Figure 6.20: Original measured S21 of chipB vs. improved extracted RLC model S21.................... 138

Figure 6.21: Improved extracted series RLC model of chipC........................................................... 139

Figure 6.22: Original measured S21 of chipC vs. improved extracted RLC model S21................... 139

Figure 6.23: Error percentage for chipA. ................................................................................................ 140

Figure 6.24: Error percentage for chipB............................................................................................................ 140

Figure 6.25: Error percentage for chipC............................................................................................... 141

Figure 6.26: New extracted RLC model of chipC....................................................................... 143

Figure 6.27: Original measured S21 of chipC vs. new extracted RLC model S21............................ 144

Figure 6.28: Error percentage for chipC............................................................................... 144

Figure 7.1: Simplified clock distribution circuits for a DDR controller based on a PLL or an MDLL.

Figure 7.2: The testbench of sizing DECAP to satisfy the supply noise specification........................ 148

Figure 7.3: Input supply voltage VDDin and on-die supply voltage................................................. 149

Figure 7.4: Input supply voltage VDDin and on-die supply voltage..................................................... 150

Figure 7.5: PSIJ sequences calculated through PSIJS of Buffer chain 4 calculated with equation

Figure 7.6: Jitter sequences plots at the output of Buffer chain and at the output of PLL................ 154

Figure 7.7: Jitter sequences plots at the output of Buffer chain and at the output of MDLL............ 154

Figure 7.8: The on-die supply voltage with the $\mathrm{ESC} / \mathrm{ESR}=690 \mathrm{pF} / 0.52 \Omega$ and with the equivalent broadband equivalent circuit as the Intrinsic ODC model.................................................... 155

Figure 7.9: Buffer chain PSIJ sequence simulated with $\mathrm{ESC} / \mathrm{ESR}=690 \mathrm{pF} / 0.52 \Omega$ and the equivalent broadband equivalent circuit as the Intrinsic ODC model 155 
Figure 7.10: Buffer chain PSIJ sequence simulated with the supply voltage profile VDDdie_mdll as shown in Figure 7.4 ......................................................................................................... 155 


\section{Chapter 1}

\section{Introduction}

\subsection{Motivation}

With technology scaling and increasing data rates, in modern integrated circuits, the average current density and power noise amplitude have increased due to decreasing power supply voltage and increasing switching speed. Reduced supply voltage causes reduced noise margins, large voltage variations and other signal integrity issues which could lead to design failures [1].

Power supply noise is one of the major sources of timing jitter [2]. It directly contributes to the jitter of the internal timing sources of the system, e.g., PLL (phase locked loop), DLL (delay locked loop) and clock distribution buffers [2]. Jitter is a non-linear function of the magnitude and frequency of power supply noise. There is a band of power supply noise frequency that can create the worst type of jitter, the center frequency of which varies with its amplitude [1].

\subsubsection{Power Supply Noise}

A power distribution network (PDN) is the network that connects the power supply to 
the power/ground terminals of the on-die circuits. A simplified PDN is shown in Figure 1.1. Ideally, it provides sufficient voltage and current for the circuits when the transistors switch. However, the inductance and resistance of the PDN prevents supplying an infinitely large amount of current in an infinitesimally small amount of time. Mainly the package inductance induces undesired voltage fluctuation, $\Delta \mathrm{V}$, through the mechanism of $\Delta V=L(d i / d t)$ [3], so that the current transients on the PDN cause power supply noise. To solve this issue, in the past few years many studies on power supply induced jitter (PSIJ) have been published [4-10].

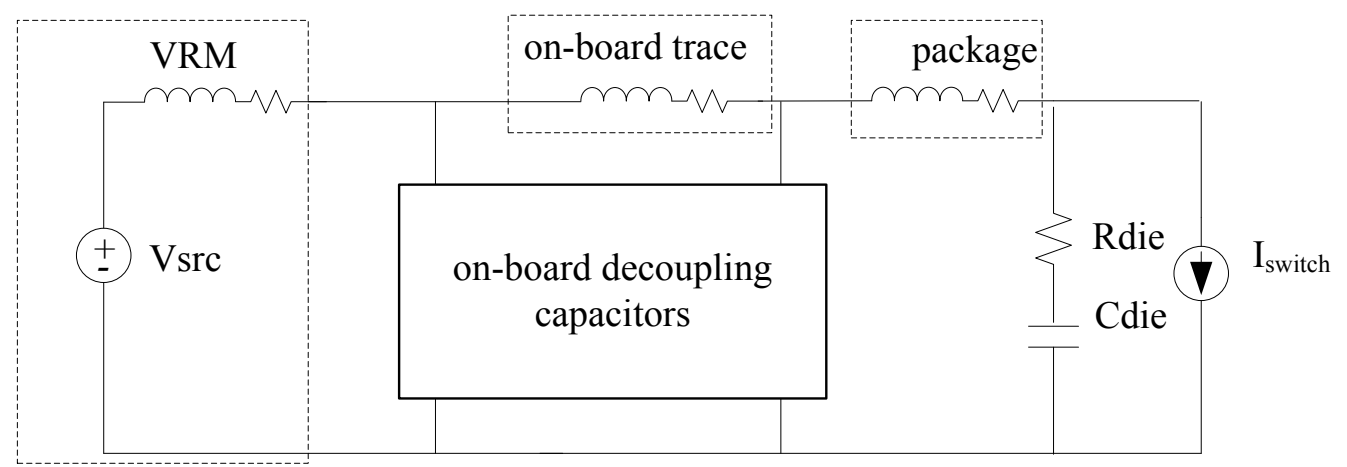

Figure 1.1: A simplified PDN for on-die circuits.

\subsubsection{DDR Controller and Design Challenges}

Synchronous Dynamic Random Access Memory (SDRAM) is popular due to its high performance and density. SDRAM can be divided into two primary categories. One is Double Date Rate (DDR) SDRAM which is widely used on personal computers (PCs) and 
servers. The other one is mobile DDR (mDDR) or low-power DDR (LPDDR), which is widely used on mobile computers such as smart phones, tablets and digital cameras. SDRAM applications use high speed links to connect a processor to the memory. The core of the interface is the DDR controller. A simple DDR interface block diagram is shown in Figure 1.2.

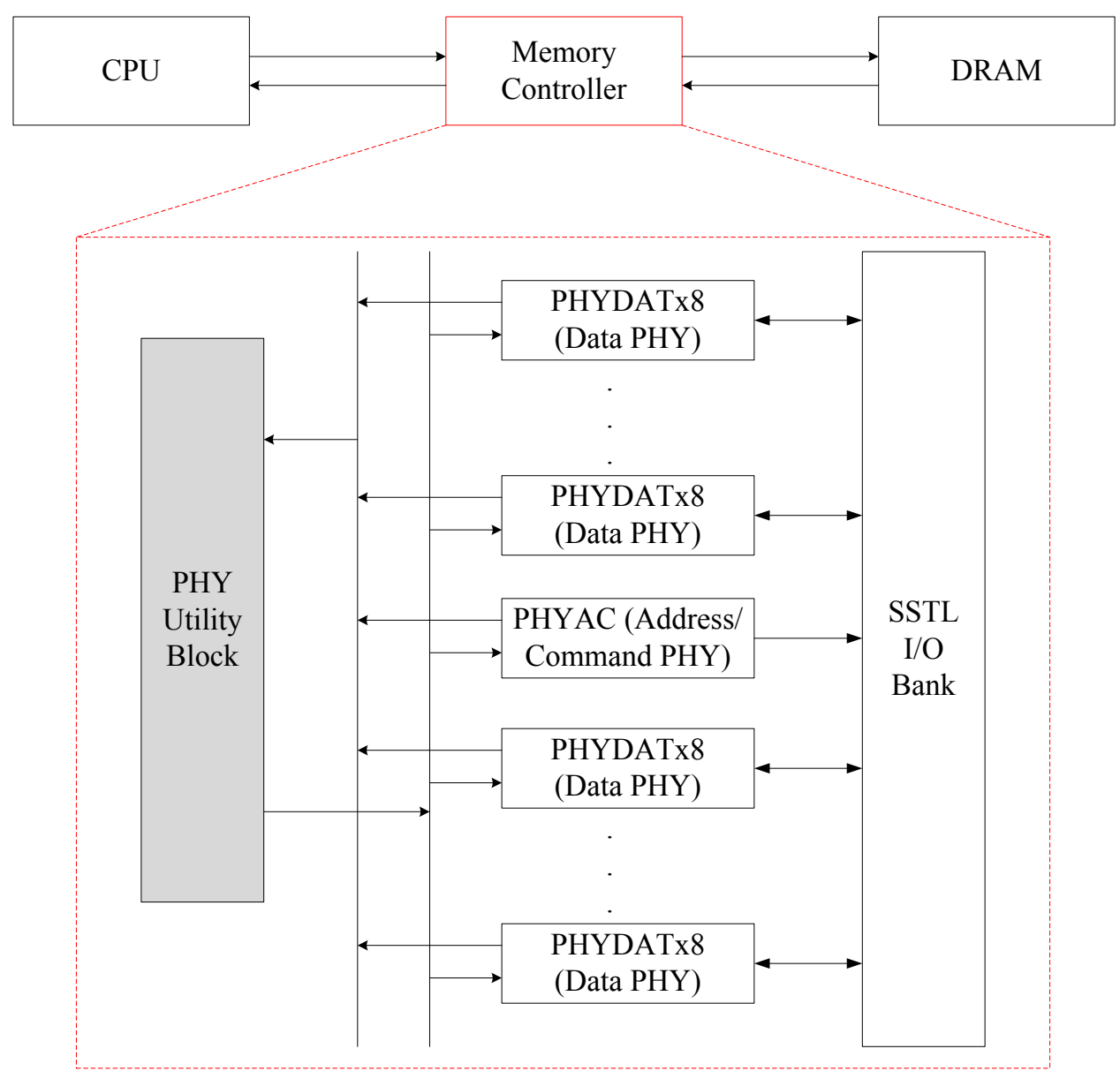

Figure 1.2: DDR interface block diagram.

As data rate increases, designers of memory controllers face many challenges including 
high pin count, tight packaging constraints, and single-ended signaling. Single-ended signaling, one of the most critical challenges, is vulnerable to simultaneous switching noise (SSN). SSN happens when multiple output drivers on a chip switch at the same time and in the same direction. This causes a huge increase in the current on either the power or ground net and induces a voltage drop in the chip/package power distribution, $\Delta \mathrm{V}$, through the same mechanism of $\Delta V=L(d i / d t)$. The resulting value of $\mathrm{di} / \mathrm{dt}$ is cumulative and increases with the number of simultaneous switching outputs (SSOs); the higher di/dt the higher the $\Delta \mathrm{V}$ amplitude [11]. This is a common problem on systems with a wide parallel address or data buses.

The current data rate of DDR3/LPDDR3 ranges from $800 \mathrm{Mbps}$ to $2133 \mathrm{Mbps}$, and the rate of DDR4 ranges from $2133 \mathrm{Mbps}$ to 3200Mbps. LPDDR4 starts from $2133 \mathrm{Mbps}$ and can go up to 4266Mbps. A 64-bit DDR bus may have more than 110 pins. All these constraints in high pin count and high speed limit the SSN performance while the SSN directly impacts the PDN.

This research focuses on the methods for estimating and improving PSIJ performance within the application of a double data rate (DDR) controller. Jitter for the DDR controller is mainly caused by the clock multiplier, the clock tree, and the IO buffer [1]. On-die measurement shows that $33 \%$ of the total jitter is contributed by the clock multiplier and the IO buffer; while $67 \%$ of the total jitter comes from the clock tree [1]. The clock tree jitter is 
largely related to power supply noise.

Methods for improving system jitter performance and estimation have been deeply studied in the past decade. For example, to achieve low jitter clock multiplication, multiplying delay locked loop (MDLL) [12][13][14] have been explored. Compared to a PLL, an MDLL will be a better candidate for DDR controller applications because its superior phase noise outperforms the conventional PLL [15][16][17]. Most importantly, any jitter created by the on-chip noise is completely corrected when a clean reference clock edge arrives at the input of the DLL [18]. An MDLL achieves both low jitter and ease of design, however, its high reference spur limits its application. In this dissertation, an MDLL with static phase offset (SPO) reduction technique is proposed to overcome the limitations [19][20].

Because the physical sizes of block PHYDATx8 and PHYAC shown in Figure 1.2 are large and the capacitive load of the output of clock multipliers is typically large, long clock buffer chains [1] have been used. As a result, the PSIJ of a buffer chain becomes significant. The PSIJ must be well controlled and accurately estimated in jitter budget calculation. Conventionally a metric know as the alpha factor [21] is used to estimate the PSIJ of a buffer chain; however, as data rate approaches multi-gigabits per second, this method becomes too pessimistic.

To properly characterize the PSIJ, PSIJ analysis methodology [22][23][24][25] has been 
proposed and applied to multi-gigabit I/O interface design. The key concept of power supply induced jitter sensitivity (PSIJS) has been proposed and applied to PSIJ analysis. PSIJS of single buffer [26][27] and PSIJ of two- stage [28] and multi-stage [29] buffers have been studied. In this dissertation a simple and concise PSIJS expression of multi-stage buffer chain [30] is derived, which can be used to calculate PSIJ of buffer chains quickly and accurately.

To decrease PSIJ, an on-die decoupling capacitor (ODC) is often the most effective method, but over-design can increase the cost and sometimes make the method unfeasible. An ODC can be modeled as a resistor in series with a capacitor. The series resistor and the series capacitor are respectively called Rdie and Cdie.

From the die perspective, in the frequency range between $50 \mathrm{MHz}$ and $500 \mathrm{MHz}$, the parallel Cdie and the package inductance form a resonant impedance peak on the power distribution network (PDN), which may cause a large IR (where $\mathrm{R}$ is the supply rail resistance and $\mathrm{I}$ is the current flowing through the resistance) drop so that the resonant frequency should be accurately calculated to evaluate the effect of the IR drop. A method of accurately obtaining the frequency domain (narrowband) ODC is reported in [31]. However, the frequency domain ODC cannot be directly used for the time domain transient simulation applications, which include plotting time domain waveforms for jitter estimation, and sizing how much extra on-die decoupling capacitance is needed to satisfy the supply noise design 
specification which typically is less than $\pm 5 \%$ nominal supply voltage. In order to overcome the above difficulties, in this dissertation, an accurate conversion method is proposed for frequency domain narrowband to time domain wideband Rdie/Cdie and the effectiveness is verified with a test chip measurement.

\subsection{Contributions to Knowledge}

The main contributions to knowledge of this dissertation are summarized as follows,

1. An MDLL with a novel SPO reduction technique is proposed and verified at the transistor level. A Simulink model of an equivalent MDLL with the proposed SPO reduction technique is also created to verify the efficiency in reducing reference spurs.

2. Equation (4.22), a very simple and highly accurate expression of PSIJS transfer function for a CMOS buffer chain, is proposed and verified. The transfer function is mainly a function of the maximum and minimum propagation delay of the buffer chain. The function can be easily obtained and used in jitter budget calculation.

3. A concise method is proposed and verified to convert the frequency domain narrowband ODC (including Rdie and Cdie) to an approximate broadband equivalent circuit.

\subsection{Document Organization}

This document is organized as follows. Chapter 2 reviews recently reported MDLLs 
focusing on cyclic reference injection based ones. In Chapter 3, an MDLL with SPO reduction technique is proposed. The efficiency of reference spur reduction is verified at the behavioral level of the Simulink model of the equivalent MDLL and at the transistor level of the proposed MDLL. In chapter 4, the analytical expression of power supply induced jitter sensitivities of a buffer chain is derived, analyzed and compared with simulation results. Chapter 5 presents the proposed method of converting narrowband Rdie and Cdie to an approximate broadband equivalent circuit. In chapter 6 , the measurement method and results of Rdie and Cdie of three test chips are presented. Chapter 7 presents a case when the proposed techniques are applied to a design of the clock distribution circuit for a DDR controller. This chapter also summarizes the previous chapters, specifies the main contributions to knowledge, and identifies directions for future research. 


\section{Chapter 2}

\section{Overview of DLL-based Frequency Multipliers}

\subsection{Introduction}

Higher data rate applications require higher performance clock synthesizers [32]. Delay locked loop (DLL) based frequency multipliers have been widely used in interchip communication interfaces [13][33], clock distribution networks [34] [35] and radio frequency system applications [36][37]. These functions can also be achieved by PLLs, but PLLs have several weaknesses such as the design difficulty and high-cost loop filters. In particular, the jitter accumulation phenomenon makes PLLs more susceptible to power-supply and substrate noise [38], [39]. Compared with PLLs DLLs have the advantages of ease of design, better immunity to on-chip noise, and more stability [34] [40].

Because a DDR memory interface is based on single-ended signaling, it is more susceptible to crosstalk noise and simultaneous switching noise [41]. It also suffers reflection noise due to the impedance mismatch at both driver and receiver sides. In the case of DDR controller application, DLLs are preferred because any jitter created by the on-chip noise is 
completely corrected when a clean reference clock edge arrives at the input of the DLL [18].

However, the DLL based clock multipliers have poor reference spur performance which limits their applications. This limitation has sparkled many research efforts to minimize output spurs in recent years $[13,14,15,16,42]$.

Comparison of PLL and MDLL is summarized in Table 2.1.

Table 2.1 Comparison of PLL and MDLL

\begin{tabular}{l|l}
\hline PLL & MDLL \\
\hline $\begin{array}{l}\text { VCO-- jitter accumulation } \\
\text { high-order system } \\
\text { can be unstable }\end{array}$ & VCDL-- no jitter accumulation [39] \\
first-order system [43] \\
costly to integrate LF & unconditionally stable [13] \\
less reference signal dependent & easy to design [85] \\
low reference spur & reference signal dependent [4] \\
\hline
\end{tabular}

Table 2.2 Classification of DLL based frequency multipliers

\begin{tabular}{|c|c|c|}
\hline \multirow{2}{*}{ Integer-N MDLL } & Edge combiner based & fixed-ratio \\
\cline { 3 - 3 } & $\begin{array}{c}\text { Cyclic reference } \\
\text { injection based }\end{array}$ & Programmable \\
\cline { 2 - 3 } &
\end{tabular}

Generally, reported MDLLs can be divided into two types [44], the edge combiner based MDLL and cyclic reference injection based MDLL, which are compared in Table 2.2. 
The edge combiner based MDLL can be categorized into fixed-ratio integer-N MDLL and programmable integer-N MDLL. The reported cyclic reference injection based MDLLs are all programmable.

\subsection{Edge Combining MDLL}

The basic idea of the edge combining technique is illustrated in Figure 2.1 [45].
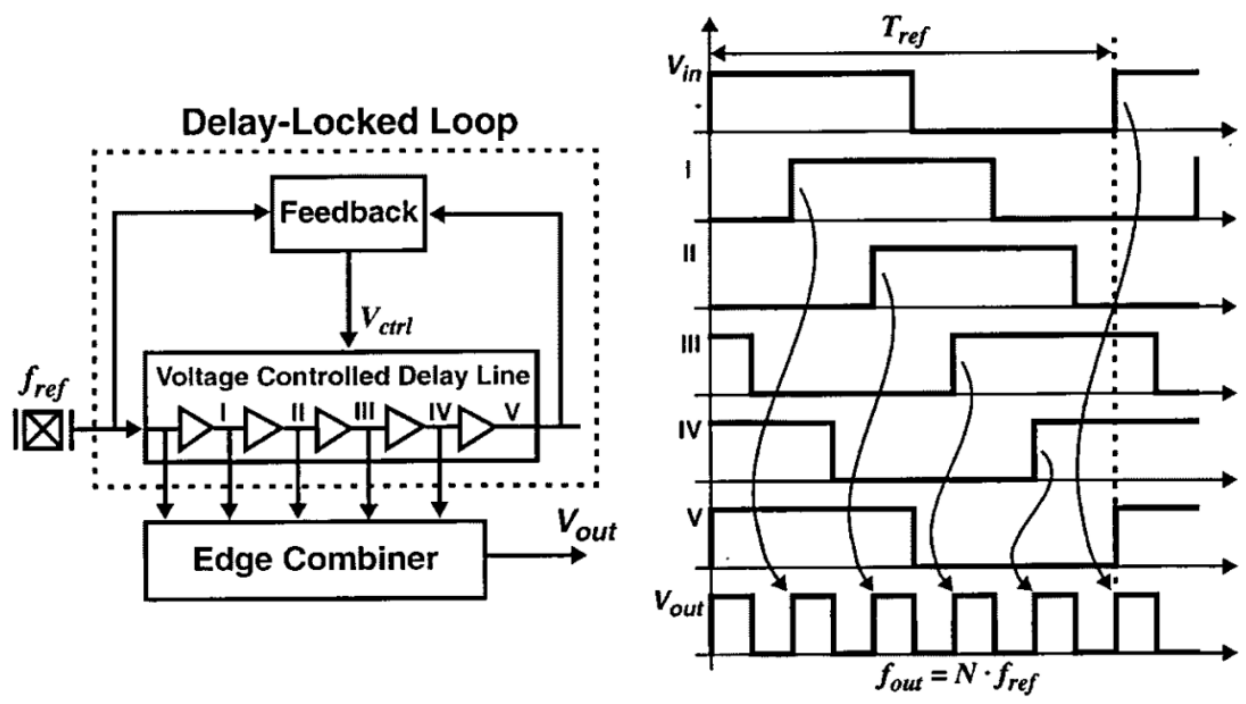

Figure 2.1: Block diagram of edge MDLL and its typical waveform [45] @ 2000 IEEE.

The low phase noise signal $f_{\text {ref }}$ is applied to a voltage controlled delay line (VCDL), and the delayed signals from delay stage I, II... and V are combined by the edge combining circuit. The delayed signal from the last stage is applied to the feedback circuitry where a control signal is generated to tune the VCDL delay value to be exactly equal to one reference period. The output signal, $V_{\text {out }}$, is the combined high-frequency signal. The delayed signals 
are shown in the figure on the right from stage $\mathrm{I}$ to $\mathrm{V}$ as an example. Within one reference period Tref, the reference signal is delayed by $\mathrm{N}$ stages with equal delay values, and each rising edge of the delayed signal is taken as the rising edge of the output to obtain the final output with the frequency $\mathrm{N}$ times the $f_{\text {ref, }}$, where $\mathrm{N}$ is 5 in this example. So more delay stages mean a higher combined frequency. By selecting different delay signals and feeding them to the feedback circuitry, different multiplication ratios can be obtained. For example, the signal III is selected and then applied to the feedback circuitry. When the edges of stage I, II and III are combined, an output frequency 3 times of the reference is generated. Based on whether the multiplication ratio is fixed or programmable, the edge combining frequency multipliers are categorized into fixed ratio category and programmable ratio category.

The edge combining MDLL in Figure 2.1 suffers from two major drawbacks. The first drawback is that any mismatch in the delay stages or the edge combining logic translates directly into duty cycle error and fixed-pattern jitter, which results in spurious tones around the desired output frequency. The mismatch can only be reduced by accurately calibrating the delay of each delay stage, which means a higher complexity of circuit and power consumption. The second drawback is that it is difficult to realize a programmable clock multiplication ratio. This is because that selecting different delay stages needs different multiplexers (MUXes); besides, more output frequencies need more MUXes and more complex control circuitry [46][47]. The mismatch among different signal combining paths 
(i.e., different delay stages and MUXes) results in large spurs at the output (e.g. -20dB at reference frequency with the carrier frequency of $1.2 \mathrm{GHz}[48])$.

\subsection{Cyclic Reference Injection MDLL}

In the previous sections, edge combining MDLL circuits were discussed. One of the drawbacks is that it is difficult to realize a programmable multiplication ratio. Another drawback is that the mismatches in the delay stages and edge-combining logic cause significant deterministic timing jitter at the output. Bearing this in mind, this section reviews recently reported MDLL frequency multipliers with the cyclic reference injection technique. This type of MDLL can easily achieve programmable multiplication and high frequency output. Another significant advantage of the cyclic reference injection over the edge combiner is that the output frequency is less sensitive to the mismatch between the VCDL and the edge combining circuitry [13], which results in large spurious output power. Because each edge of the MDLL output passes through the same delay element, the fixed pattern jitter due to mismatch among delay stages is avoided.

The conventional block diagram of a cyclic injection MDLL is shown in Figure 2.2 [43]. This MDLL consists of a PD, a charge pump, a VCDL, and loop capacitors as a conventional DLL may have. In addition, the MDLL has a MUX, a divider and a select logic to help generate high frequency output with multiple programmable ratios. Similar 
architecture can also be found in [49] [50]. With the MUX, the VCDL output is fed back to its input, and forms a ring oscillator. The high-frequency signal generated by the ring oscillator is divided by the divide-by-M divider which is essentially a counter, and the last signal is generated at every $\mathrm{M}$ cycles of the ring oscillator. The last signal triggers the select logic that switches the MUX input to inject the clean reference signal to the delay line. The ring oscillator then works as a voltage controlled delay line, and the clean reference edge resets the phase noise accumulated in the previous M-1 cycles. The function of periodically reference injection into the ring oscillator removes the accumulated phase noise, and continuous integer $\mathrm{N}$ multiple ratio can be achieved in the conventional counter, whose output frequency equals $\mathrm{N}$ times the reference. 


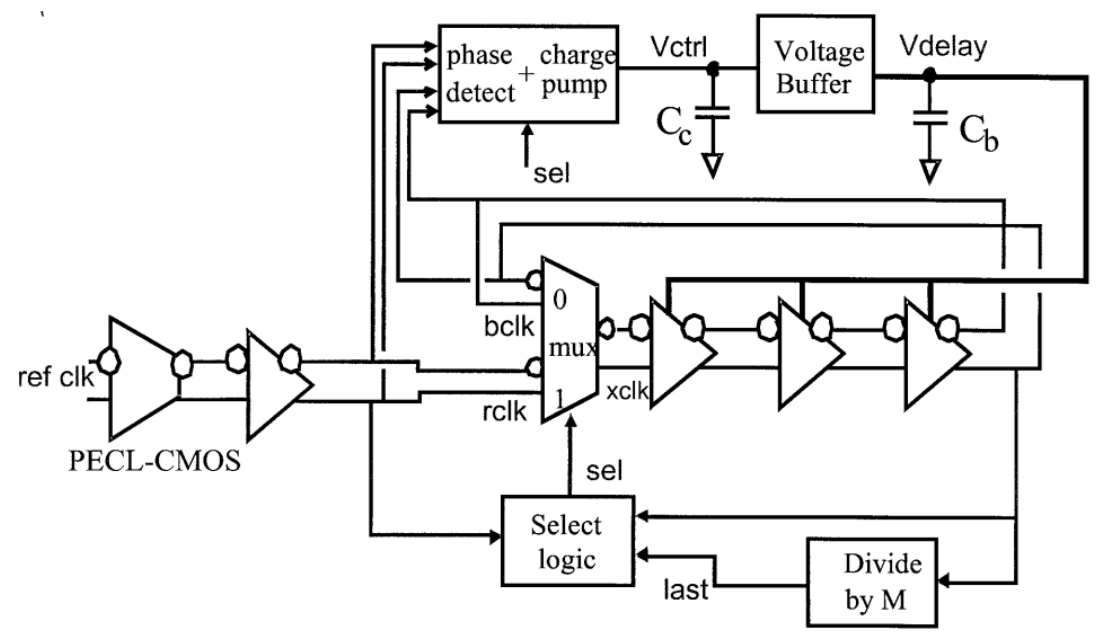

Figure 2.2: Block diagram of the MDLL with cyclic reference injection [43] (C) 2002 IEEE.

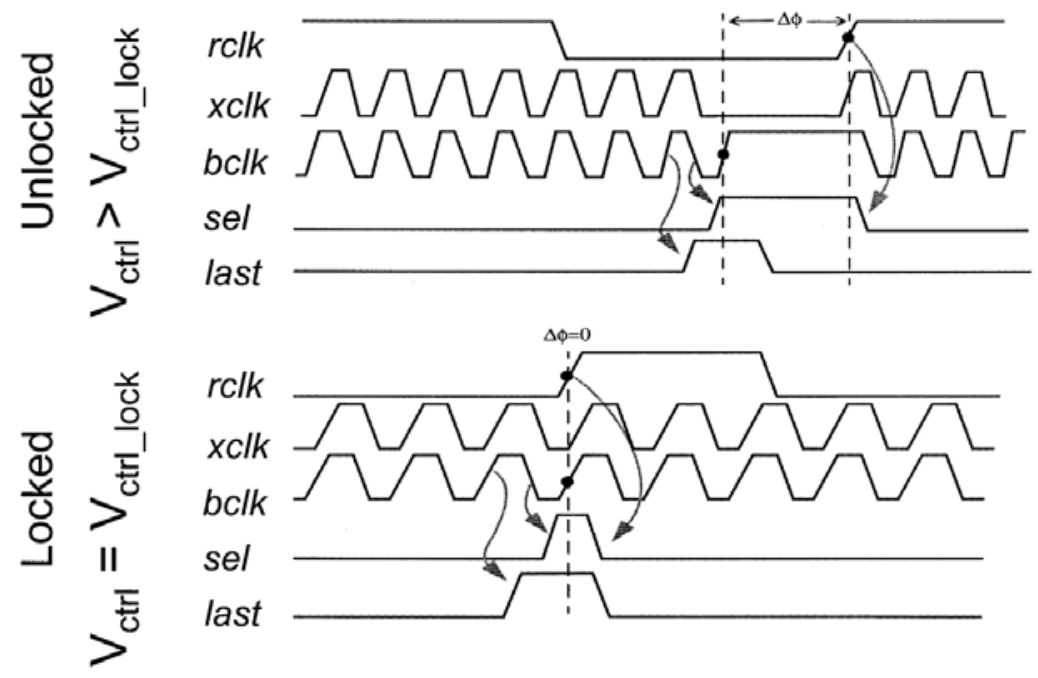

Figure 2.3: Frequency multiplier timing [43] (C) 2002 IEEE.

Figure 2.3 shows the detailed timing diagram of the MDLL. As the circuit powers on, the loop control voltage is pulled high, the delay line is set to its minimum value and the oscillating frequency is set to its maximum frequency. As a result, the $b c l k$ signal completes $\mathrm{M}$ cycles before the following reference rising edge $r c l k$. On the $M$ th rising edge of $b c l k$, the 
divider enables the select logic which inserts the sel signal at the following falling edge of $b c l k$, and switches the multiplexer to $r c l k$, so a clean reference is injected. At this point, the reference signal propagates in the VCDL, and MUX is kept connected to $r c l k$. The phase detector (PD) compares the corresponding rising edge of $b c l k$ with that of the $r c l k$ which is newly injected and a phase error $\Delta \phi$ is generated. After the reference is injected, the select logic disables the last signal, and the MUX is switched to bclk, restarting the oscillator. The phase error drives the charge pump and the loop filter to decrease the control voltage and the oscillating frequency until the loop is in lock as shown in the figure when the $V_{\text {ctrl }}$ equals the $V_{\text {ctrl-lock. }}$

The main advantage of this architecture is that it combines the low phase noise of a DLL with the programmable multiplication ratio of a PLL. The main drawback is that the process of injecting the reference edge introduces additional phase error that can be hardly detected by the conventional phase detector and charge pump, and the phase error causes spurious tones in the output spectrum. For example, in [44], without the spur reduction technique, the MDLL is measured with $-23 \mathrm{dBc}$ at $1.2 \mathrm{GHz}$, and the phase noise is $-110 \mathrm{dBc} / \mathrm{Hz}$ at $100 \mathrm{kHz}$. 


\subsection{Overview of Reported Spur Reduction Techniques for Cyclic Reference Injection MDLL}

The relatively high reference spur power in the spectrum of the output is the main drawback of the cyclic reference injection MDLL, which limits its wide applications. In this section, the techniques for reducing the spur power are overviewed.

In the design of cyclic reference injection MDLL, there are many phase noise and timing jitter sources. The main sources include the static phase offset due to the mismatch in the charging and discharging current sources in the charge pump, leakage current which might be caused by the charge pump itself, by the on-chip varactor, or by any leakage on the control path from the charge pump to VCO, the timing mismatch in the PFD, clock feed-through, charge sharing of $U P$ and $D N$ switches of the charge pump, and the position of the selection window of reference injection edge [14].

In the past decade, many designs reported in literature have employed some techniques to lower the reference spurs in cyclic reference injection MDLL [13,14,15,16,42].

In [13], the static phase offset in the phase detector is compensated. A low-bandwidth secondary loop, called period error compensation loop (PECL), is added to compensate the current of the charge pump. In the PECL, an error detector (ED) has been proposed. The block diagram is shown in Figure 2.4. The main loop is very similar to the one used in Figure 
2.2 in [43].

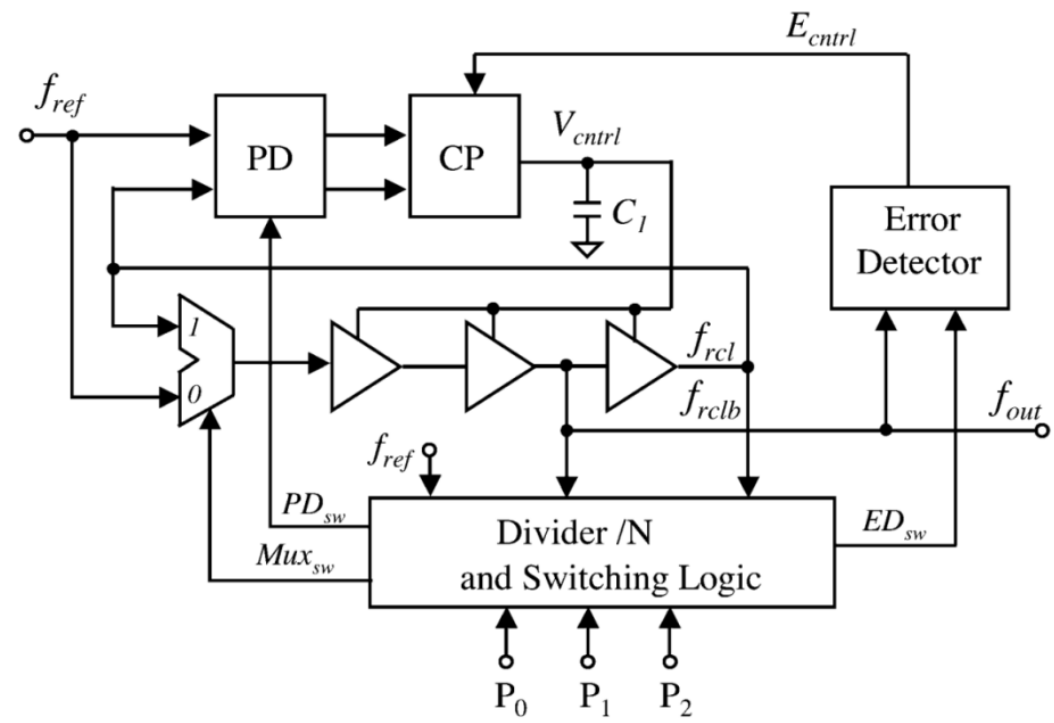

Figure 2.4: Block diagram of cyclic reference injection MDLL with period error compensation loop [13] (C) 2006 IEEE.

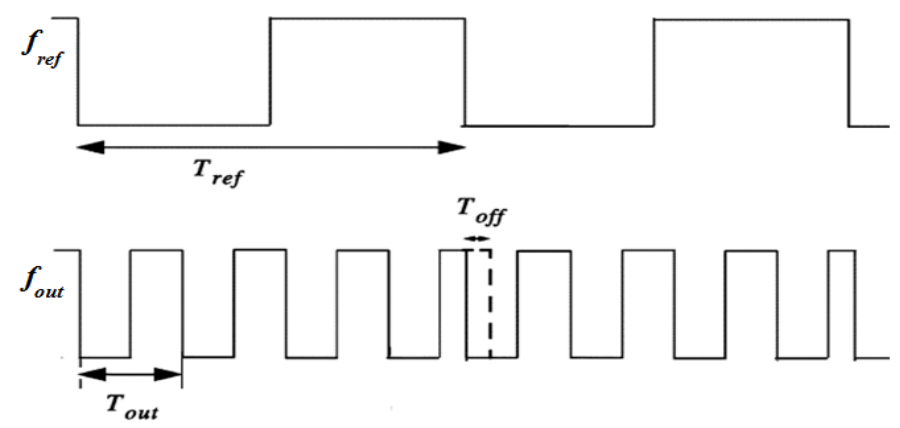

Figure 2.5: The $\mathrm{N}$-th $f_{\text {out }}$ cycle has a significant different period compared with the rest cycles of $f_{\text {out }}$ due to the static phase offset in the PFD.

Due to the static phase offset in the phase frequency detector (PFD), the N-th cycle always has its period different from the periods of the previous $\mathrm{N}-1$ cycles (as shown in Figure 2.5) even when the loop is in lock, this leads to large output spurs. This static phase offset is referred to as the period error in the output of the VCDL. To minimize the error, the 
output $f_{\text {out }}$ is applied to an ED to extract this error and compensate it by tuning the charge pump current. The ED compares the period of the Nth output cycle, $T_{\text {out }}(n)$, with the average period, $T_{\mathrm{av}}$. The difference between $T_{\text {out }}(n)$ and $T_{\mathrm{av}}$ is amplified. In this design, besides the loop governed by the PFD, which dynamically compensates for the period error, a low bandwidth PECL, governed by the ED, is used to compensate for the static period error between $T_{\text {out }}(n)$ and $T_{\text {av }}$.

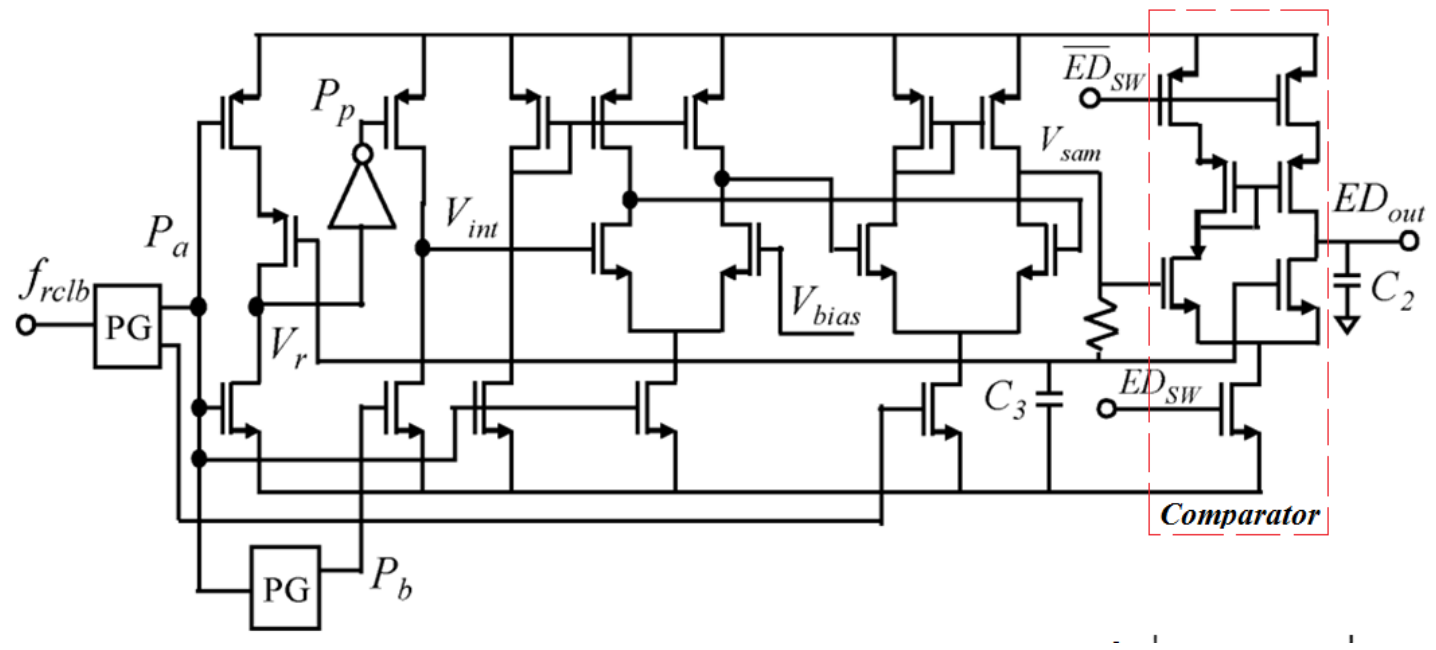

Figure 2.6: CMOS implementation of the ED [13] C 2006 IEEE.

The schematic of the ED is shown in Figure 2.6 and its operation, for the case of positive period error, is illustrated in Figure 2.7. At each falling edge of the input signal $f_{\text {rclb }}$, the pulse generator (PG) generates a narrow pulse, $P_{a}$, the width of which is then increased by an amount corresponding to the average period duration. The resulted pulse train, $P_{p}$, is 
inverted and integrated by a high-gain integrator whose output, $V_{\text {int }}$, is sampled and then reset by $P_{b}$ before the next pulse comes.

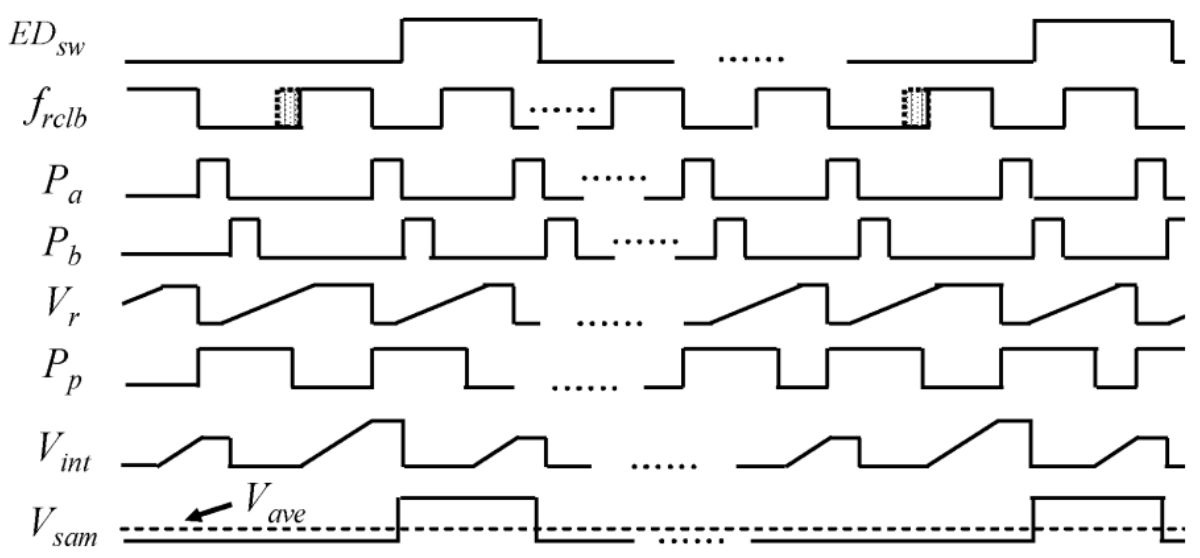

Figure 2.7: Operation of the ED [13] C) 2006 IEEE.

The sampled result, $V_{\text {sam }}$, corresponding to the VCO periods, drives the internal feedback, so that the average value $\left(V_{\text {ave }}\right)$ of the sampled results $V_{\text {sam }}$ can be dynamically maintained by the internal feedback at a level determined by $V_{\text {bias }}$. The internal feedback loop compares $V_{\text {ave }}$ with a pre-defined value ( $\left.V_{\text {bias }}\right)$ and adjusts the width increase amount of signal $P_{a}$ accordingly. With the internal loop, a large integrator gain can be used to achieve high period error resolution. The bias voltage $\left(V_{\text {bias }}\right)$, which determines $V_{\text {ave }}$, is set to be approximately to $V D D / 2$ to ensure that the integrator works in its high-gain region. When signal $E D_{\mathrm{sw}}$ is high, a comparator is switched on to compare the samples $\left(V_{\text {sam }}\right)$ with its average $\left(V_{\text {ave }}\right)$ to generate the error output signal. Because the output of the comparator directly compensates for the static phase offset, any mismatch in the comparator will affect 
the compensation and this is not compensated.

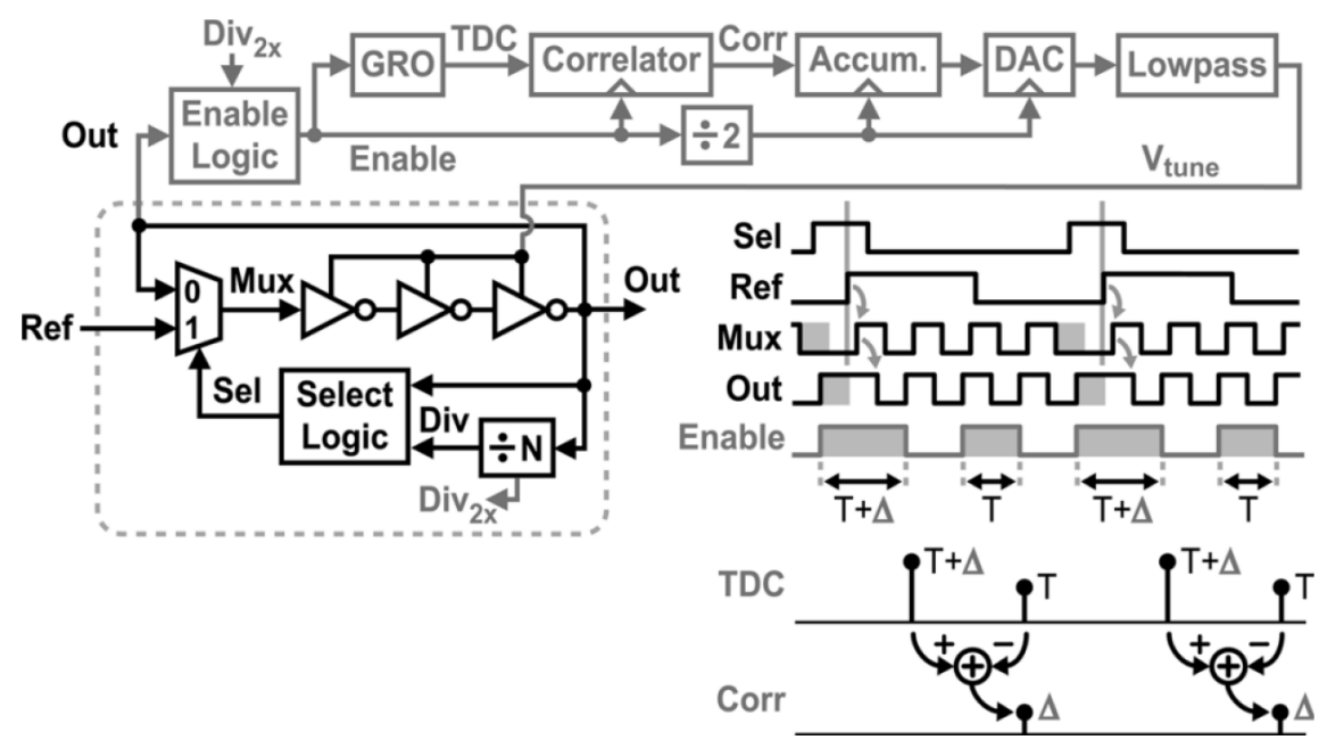

Figure 2.8: Block diagram of MDLL and tuning method leveraging a TDC and correlated double-sampling [15] C 2008 IEEE.

A highly digital MDLL is proposed with a reference spur reduction technique which is referred to as correlated double-sampling [15]. It is also based on the observation that the period of the $N$ th cycle of the output of the VCDL is always different from periods of the previous $N-1$ cycles even when the loop is in lock [13][15]. In Figure 2.8, the output signal of VCDL out has been divided by 2 to generate signal, Enable, whose pulse-width alternates twice every reference period between the instantaneous period of the oscillator, $T$, and the period of the error-affected cycle, $T+\Delta$. By doing a relative comparison of each consecutive pulse period of the Enable signal, the value of $\Delta$ can be obtained. Because only one signal is examined, the issue of a due-path mismatch is greatly alleviated. 
To realize the comparison of $T$, and $T+\Delta$, a gated ring oscillator (GRO) time to digital converter (TDC) has been used. The GRO block outputs a digital signal, TDC, which is updated at the end of each Enable pulse and corresponds to a quantized measurement of the corresponding Enable pulse period (i.e., $T$ or $T+\Delta$ ). A digital correlator block simply subtracts consecutive pairs of the TDC samples to yield a stream of samples, Corr, which correspond to quantized estimates of $\Delta$. By passing these $\Delta$ samples into a digital accumulator, Accum., the quantization error of the $\Delta$ samples is reduced by the averaging effect of the accumulation operation (assuming that the quantization error varies in an appropriately random fashion). The accumulator output adjusts $V_{\text {tune }}$ through a digital to analog converter (DAC) with an accompanying RC low-pass filter until $\Delta$ reaches zero at steady state.

Figure 2.9 displays a conceptual diagram of the GRO TDC used to measure the time duration of the oscillator cycles. The key operating principle is to turn on a ring oscillator during the time measurement period (i.e., assert the Enable signal as shown in the figure) and count how many edges occur among the various oscillator stages. As such, the time resolution of the measurement corresponds to the delay per stage of the oscillator, and quantization error occurs at both the beginning and end of the measurement as indicated in the figure. 

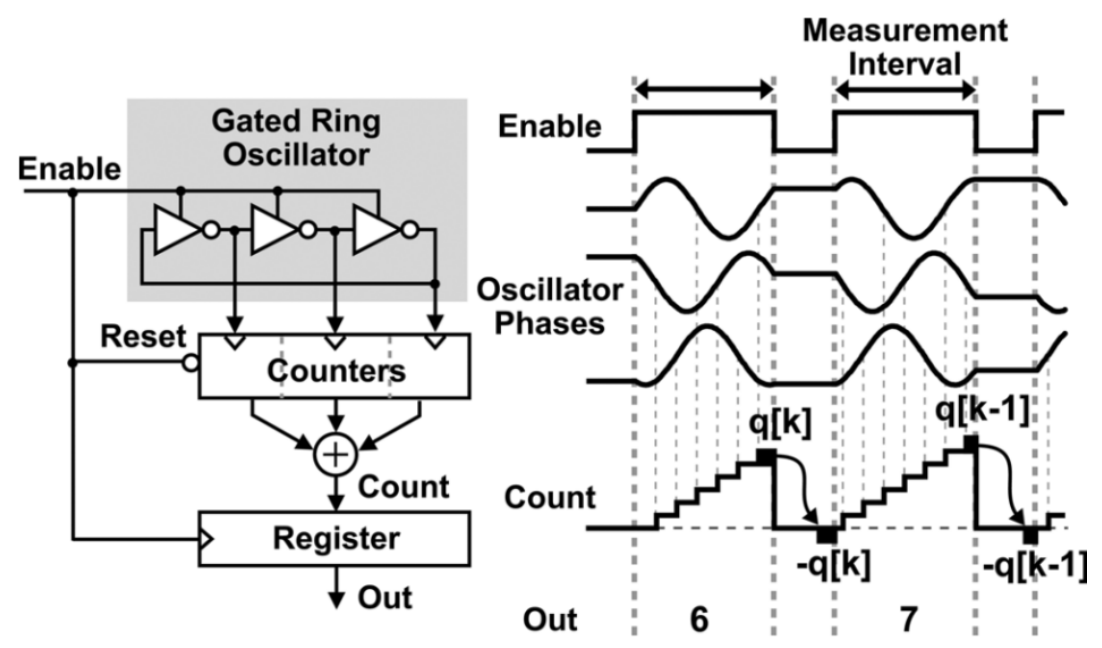

Figure 2.9: Gated ring oscillator time-to-digital converter [15] (C) 2008 IEEE.

In [15], the same assumption as in [13] is adopted: the previous $\mathrm{N}-1$ cycles of the signal $f_{\text {out }}$ have the same period as $T_{\text {out }}$, and the Nth cycle with the injected clean reference edge has a period smaller or larger than $T_{\text {out }}$. Because the architecture employs the double-sampling correlator as the phase detector, except for partial leakage current, the main nonidealities in the control path of a conventional MDLL are avoided. However, because a GRO TDC is used to detect the static phase error the spur performance of the MDLL is completely dependent on the performance of TDC. Although a GRO TDC has a higher resolution compared with other types (e.g., delay stage chain TDC), its inherent dead zone issue has not been solved completely and is still under exploration. The measured results of the MDLL are obtained by manually adjusting the GRO TDC to overcome the dead zone issue. So the proposed technique in [15] still needs further exploration. In addition to this, a 
high-resolution GRO TDC consumes high power.

A reference spur reduction technique called an aperture tuning mechanism is proposed in [14]. Figure 2.10 shows the MDLL architecture. In a conventional MDLL circuit, a control block generates an SEL pulse to briefly open a time window for reference injection. A key characteristic attribute of the SEL pulse is that it is sufficiently sharp and well-positioned to select the next reference edge. However, at high frequencies, the position of the SEL pulse impacts the delay of the MUX and hence introduces pattern jitter and spurs. This design minimizes the spur by introducing a calibrated phase delay to properly position the SEL pulse with respect to the reference edge, and by minimizing the inherent error of the charge pump (CP).

The MDLL shown in Figure 2.10 adds a $360^{\circ}$ phase interpolator to vary the delay of the SEL pulse by up to a VCO's clock cycle. This adjustment range compensates for any latency in the select logic generation and enables precise positioning of the SEL pulse. The VCO comprises four delay stages. A MUX stage configured as a delay cell serves as the VCO's third stage in order to match the first stage, thus the MUX stage maintains a clean quadrature relationship and ensures more linear phase steps of the SEL pulse tuning. A calibration loop uses the duty-cycle error of the VCO output (similar to [15]) to advance the phase interpolator setting toward minimal duty cycle error, which corresponds to the optimal time window position. 


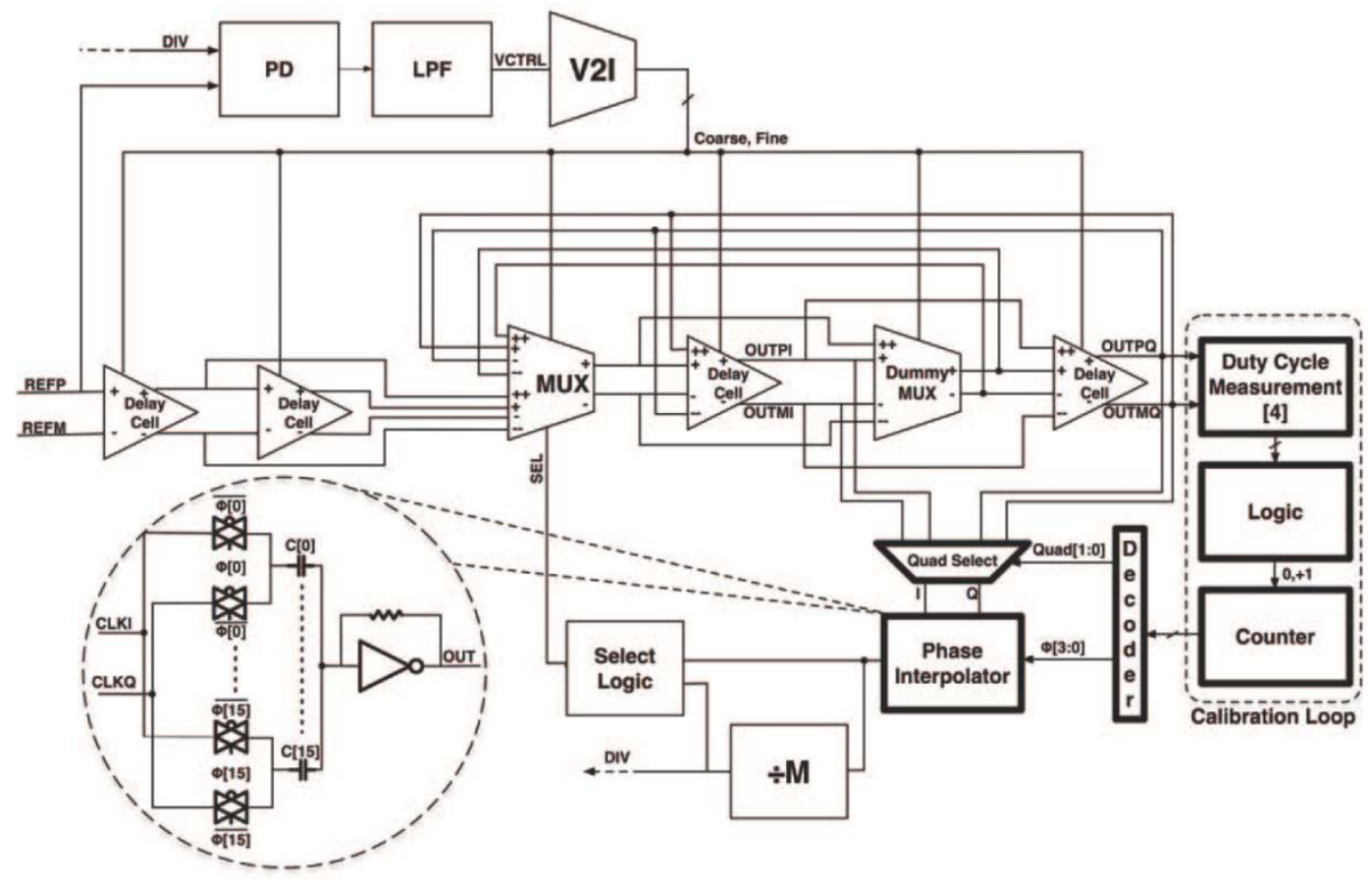

Figure 2.10: MDLL with proposed aperture tuning mechanism [14] (C) 2011 IEEE.

The phase interpolator design is shown in the inset of Figure 2.10. The interpolation takes place at the common terminal of a bank of weighted capacitors. The capacitor weight determines the degree of interpolation. $4 \mathrm{fF}$ unit capacitors are used to achieve 4 bit matching while maintaining low power dissipation.

The main contribution of [14] is that a new mechanism of static phase offset has been found and a compensation method is proposed. A charge pump with static/dynamic offset compensation and leakage compensation circuits is also proposed. However, the proposed circuit is quite complex.

Figure 2.11 shows the block diagram of a fractional-N MDLL [16]. The MDLL is 
realized by a multiplexer driven by a selection logic and five pseudo-differential inverter stages. The topology replaces every $\mathrm{N}$-th edge of the signal traveling through the MDLL with a clean edge of the reference refl, $\mathrm{N}$ being the division factor of the integer divider. The delay of the inverters is finely tuned by a digital phase-locked loop (PLL) based on a $1 \mathrm{~b}$ TDC. To avoid the noise contribution from the divider, the TDC directly compares the phases of the reference signal, ref2, with the output signal (out). Coarse frequency lock is achieved in background by an additional digital loop (not shown in Figure 2.11). Unfortunately, the mismatch between the two reference paths causes a phase offset between refl and ref2, which induces large jitter at the output. To compensate for this offset, two digital-to-time converters (DTCs), implemented as digitally-controlled delay stages, are added to each path and one of them is regulated by the control loop, based on the time error detected by the $1 \mathrm{~b}$ TDC. In contrast to PLLs, fractional-N operation cannot be achieved in MDLLs by simply dithering the modulus control (MC) of the integer-N divider via a $\Delta \Sigma$ modulator and subtracting the quantization noise from the loop. In fact, ref and out would not be aligned and no clean edge would be available to replace the edge of the signal traveling in the MDLL. This issue is solved here by subtracting the time error induced by $\Delta \Sigma$ quantization error from both refl and ref2 by means of the two DTC blocks. The amplitude of the digital input of the DTCs is regulated by a least-mean square (LMS) loop. 


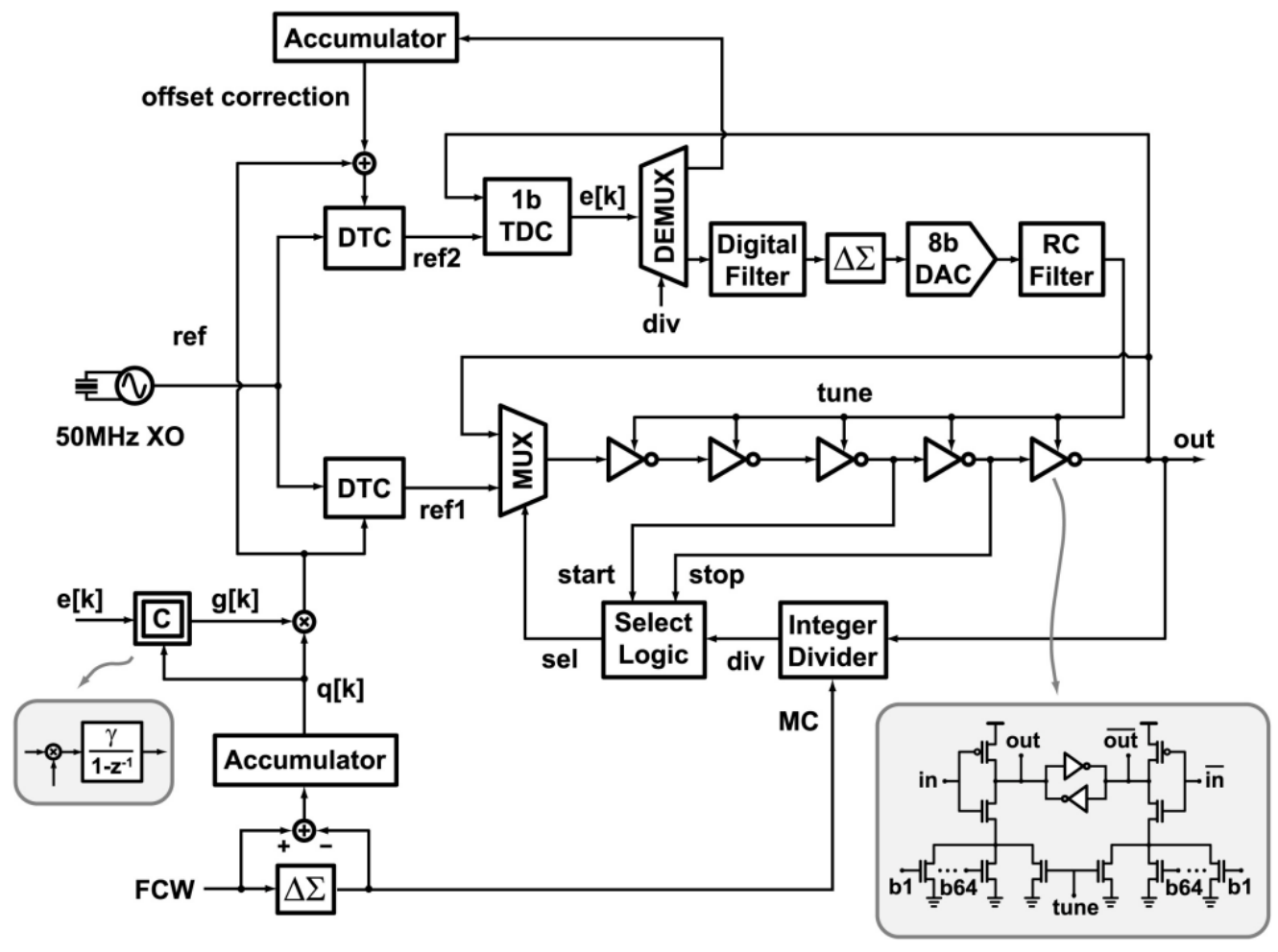

Figure 2.11: A fractional-N MDLL [16] C 2014 IEEE.

The main drawback of this design is that the performance is highly dependent on the high-resolution DTC which also consumes high power.

A low reference spur digital MDLL is reported in [42]. The block diagram of the proposed digital MDLL is shown in Figure 2.12. It consists of a separate frequency-locking loop (FLL) and a type-I multiplying delay-locked loop. The FLL, composed of a frequency detector and accumulator, drives a digitally-controlled multiplexed ring oscillator (DXRO) toward frequency lock. Unlike traditional MDLLs whose performance is limited by mismatch between the phase detector and the charge pump, the proposed digital MDLL 
tuning loop utilizes a 1-bit TDC and a digital loop filter (DLF) to drive the digitally-controlled multiplexed ring oscillator (DXRO) toward the phase lock. The 1-bit TDC, as opposed to the high resolution power hungry TDC, allows significant power reduction. The divider and selection logic blocks are used to reset jitter accumulation in the oscillator by periodically injecting a clean reference edge. To ensure clean reference injection, the multiplexer in the DXRO is carefully designed. A 1-to-4 demultiplexer eases the speed requirements of a fully-synthesized control logic. The effect of loop latency is minimized by feeding only the 14-MSBs of the 18-bit digital accumulator output to the DAC. The DACs are implemented with a cascade of a digital delta-sigma modulator that truncates the accumulator output and a 15-level current-mode DAC. A second order passive low-pass filter suppresses the out-of-band quantization error and guarantees precise setting of the oscillation frequency to ensure proper reference injection. 


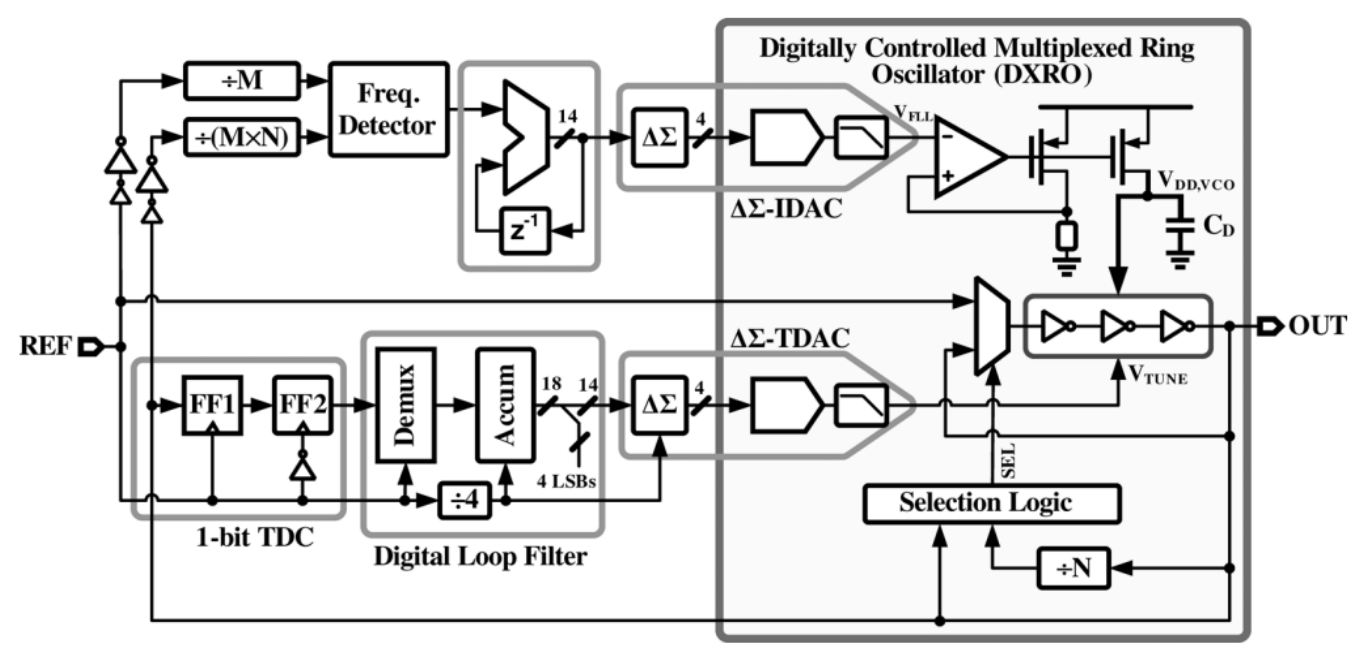

Figure 2.12: A low reference spur digital MDLL [42] C) 2013 IEEE.

\subsection{Summary}

In this chapter, first, the architecture, advantages and disadvantages of two types of MDLLs were briefly discussed. The cyclic reference injection MDLLs were shown to be more attractive than its edge combining counterparts. However, compared with the PLL frequency synthesizer, the relatively high reference spur limits the cyclic reference injection MDLL's application. After a brief discussion, the spur reduction techniques for cyclic reference injection MDLL were reviewed. 
Table 2.3 Performance comparison of selected MDLLs.

\begin{tabular}{|c|c|c|c|c|c|}
\hline & [13] & [14] & {$[15]^{*}$} & [16] & {$[42]$} \\
\hline Technology & $0.18 \mathrm{um}$ & $90 \mathrm{~nm}$ & $0.13 \mathrm{um}$ & $65 \mathrm{~nm}$ & $0.13 \mathrm{um}$ \\
\hline VDD (V) & 1.8 & 1.2 & 1.2 & N/A & 1.1 \\
\hline Output (Hz) & $0.9-2.9 \mathrm{G}$ & $4.56 \mathrm{G}$ & $1.6 \mathrm{G}$ & $1.6 \mathrm{G}-1.9 \mathrm{G}$ & $1.5 \mathrm{G}$ \\
\hline $\begin{array}{c}\text { Multiplication } \\
\text { ratio }\end{array}$ & 19 & 8 & 32 & 32 & 4 \\
\hline $\begin{array}{l}\text { Phase noise/ } \\
\text { jitter(dBc/ps) }\end{array}$ & $\begin{array}{c}\text { @ 2.16G: } \\
\text { 1.61(rms), } \\
16.9(\mathrm{p}-\mathrm{p})\end{array}$ & $\begin{array}{c}\text { @ 4.56G: } \\
\text { 2(rms), } \\
\text { 17.82(p-p) }\end{array}$ & $\begin{array}{c}\text { @ 1.6G: } \\
\text { 0.93(rms), } \\
\text { 11.1(p-p) }\end{array}$ & $1.4(\mathrm{rms})$ & $\begin{array}{l}0.9 \text { (rms) } \\
9.2(\mathrm{p}-\mathrm{p})\end{array}$ \\
\hline Spur & $\begin{array}{c}-46.5 @ \\
1.2 \mathrm{G}\end{array}$ & -46@1.6G & -58.5@1.6G & -47@1.6G & -55.6 \\
\hline Power(mW) & 19.8@2G & 6.8 & N/A & Core (3) & 0.9 \\
\hline Active area & $0.07 \mathrm{~mm}^{2}$ & $0.025 \mathrm{~mm}^{2}$ & N/A & $0.4 \mathrm{~mm}^{2}$ & $0.25 \mathrm{~mm}^{2}$ \\
\hline
\end{tabular}

* The results are measured by manually adjusting supply voltage to avoid the GRO dead zone issue.

Table 2.3 summarizes the performances of the reviewed MDLLs. In [13] a narrow bandwidth auxiliary loop is employed to compensate for the mismatch of charge pump current. The system has a potential stability problem because it has two loops. A regulator is employed in [42] to decouple the tradeoff between TDC resolution and VCO phase noise in a digital PLL design. However, since a regulator lowers the supply voltage, a level-shifter is generally needed. In addition, a regulator needs a relatively large bypass capacitor which increases the cost. In [15] the MDLL output signal first is divided by 2, and then the divided two parts are sampled by a gated ring oscillator high-resolution time to digital converter separately and finally the results are compared to generate phase error control signals. The spur performance of the MDLL is completely dependent on the performance of TDC. 
Although GRO TDC has a higher resolution, compared with other types (e.g., delay stage chain TDC), there exists the issue of the inherent dead zone which still needs to be explored. In [14], the reference spur performance is improved by optimizing the setup time of the control signal of the multiplexer and employing a reduced current mismatch charge pump. The design is too complicated for DDR controller application. A digital to time converter block has been proposed to generate fine-resolution varying reference edge to compensate for static phase offset (SPO) in a fractional-N MDLL [16]. Similar to the design in [15], the reference spur performance is highly dependent on a high resolution digital to time converter. In all digital MDLLs, the set-up time of the D flip-flop used for phase detection creates a static phase-offset between the DCO and the reference phase under the locked condition, which is very difficult to cancel [17]. 


\section{Chapter 3}

\section{Proposed Low-spur Reference Injection MDLL}

\subsection{Introduction}

In the previous chapter, types and architectures of the MDLLs and their advantages and disadvantages have been discussed. Although reported reference spur reduction techniques have improved the reference spur performance of MDLLs, the stability issue in $[13,15]$ and inherent phase offset in digital MDLLs [15,16,42] make them unsuitable for a direct application of DDR controllers.

In this dissertation, a new reference spur reduction technique is proposed, which inserts a gain stage between the PD/PFD and the charge pump to achieve lower equivalent static phase offset (SPO) which directly causes reference spurs. The proposed MDLL is verified at both the behavioral model level built in Simulink and the transistor level built up with the TSMC $0.18 \mu \mathrm{m}$ CMOS process. 


\subsection{Mechanisms of SPO}

In a PLL, SPO does not cause as many reference spurs as it does in an MDLL because in a PLL the reference edge does not replace the counterpart of the VCO output. SPOs in a PLL and an MDLL with a division ratio of $N$ that equals to 4 are shown in Figure 3.1. It can be seen that although the PLL output has SPO as shown in Figure 3.1 (b) and (c), and the period of the $N$ th cycle is almost the same as the average period of other cycles. However, compared with the PLL output, when the MDLL output has SPO as shown in Figure 3.1 (d) and (e), the period of $N$ th cycle is either larger or smaller than the average period of other cycles.

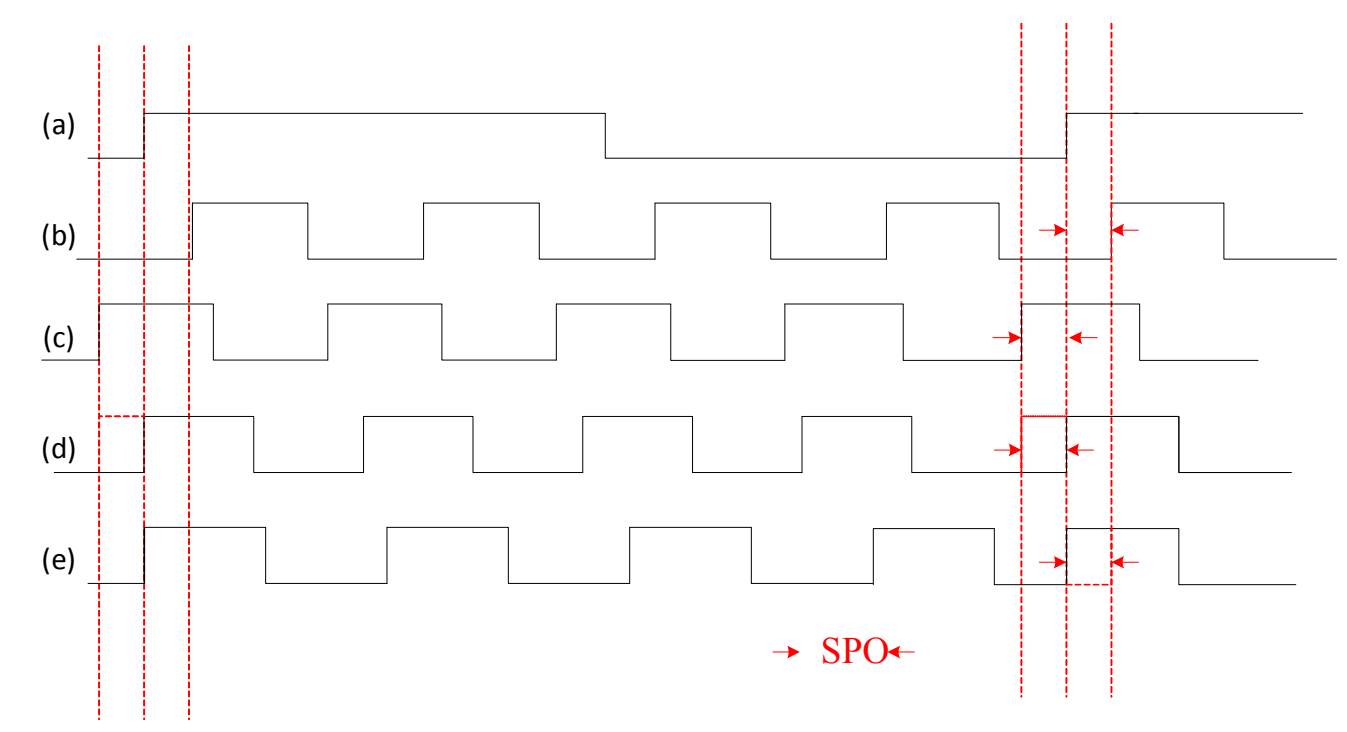

Figure 3.1: PLL/MDLL output signal in locked states. (a) PLL/MDLL reference signal; (b) PLL output with positive SPO; (c) PLL output with negative SPO; (d) MDLL output with negative SPO; (e) MDLL output with positive SPO. 


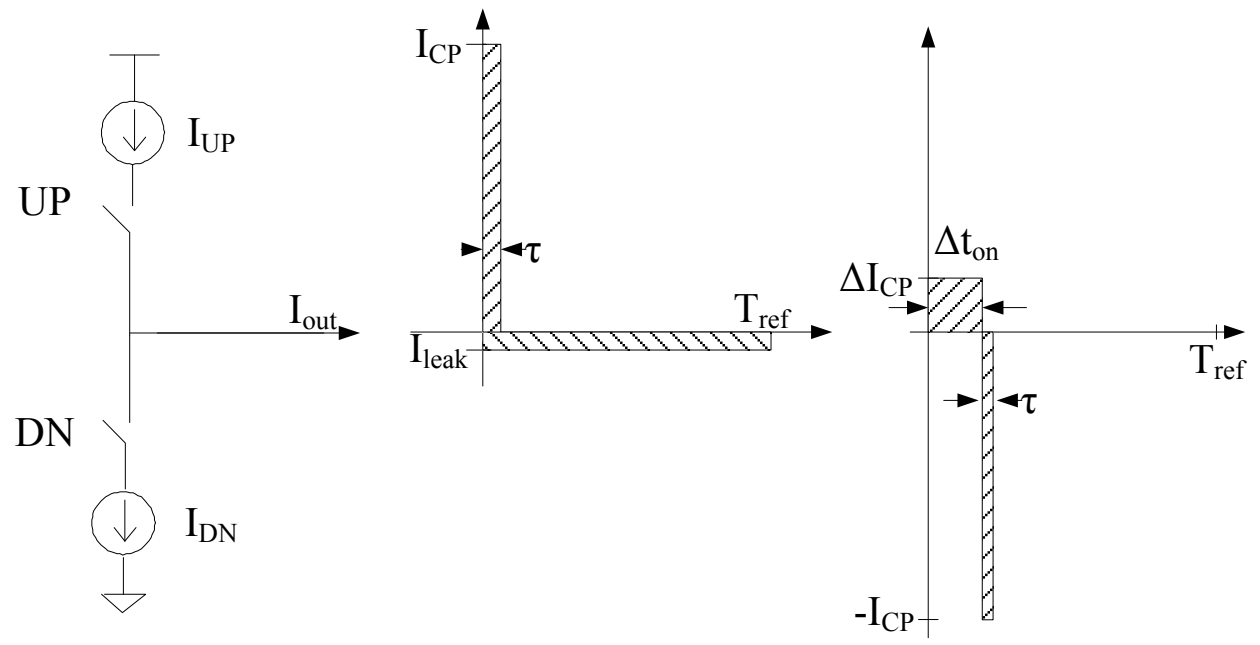

(a)

(b)

(c)

Figure 3.2: The conceptual diagram of the charge pump and charge pump current with non-ideal effects in the locked state. (a) The charge pump diagram; (b) Charge pump output current due to the current leakage; (c) Charge pump output current due to the current mismatch [52].

SPO in an MDLL is mainly caused by the current leakage on the control line and the sink and source current mismatch in a charge pump. The conceptual diagram of the charge pump is shown in Figure 3.2 (a). It consists of two switched current sources driven by a tri-state phase frequency detector (PFD). The width of the output current pulse is proportional to the phase error at the PFD inputs. Thus the phase error is proportionally converted into an amount of charge at the charge pump output.

The non-ideal effects in the charge-pump generate deterministic and periodic ripples of the control voltage [53]. The charge pump output current in the locked state with the issue of current leakage in the control line is shown in Figure 3.2 (b), where $\tau$ is the time domain static phase offset, $T_{\text {ref }}$ is the period of reference signal, $I_{\text {leak }}$ is the leakage current on the 
control line and $I_{\mathrm{CP}}$ is the average charge pump current. The SPO can be calculated in both the phase and the time domain [53][54],

$$
\begin{gathered}
\theta_{S P O}=2 \pi\left(I_{\text {leak }} / I_{C P}\right) \quad \text { or } \\
\tau=T_{\text {ref }}\left(I_{\text {leak }} / I_{C P}\right)
\end{gathered}
$$

where $\theta_{\mathrm{SPO}}$ is the SPO in the phase domain.

Figure 3.2 (c) shows the charge pump output current in the locked state with the issue of the current mismatch of $I_{\mathrm{UP}}$ and $I_{\mathrm{DN}}$, where $\Delta I_{\mathrm{CP}}$ is the current mismatch between $I_{\mathrm{UP}}$ and $I_{\mathrm{DN}}, \Delta I_{\mathrm{CP}}$ $=I_{\mathrm{UP}}-I_{\mathrm{DN}}, \Delta \mathrm{t}_{\mathrm{on}}$ is the turn-on time of the PFD that is equivalent to the minimum pulse width of the PFD output to avoid the dead-zone. In this scenario, the SPO can be calculated in both the phase and the time domain,

$$
\begin{aligned}
& \theta_{S P O}=2 \pi \frac{\Delta t_{o n}}{T_{\text {ref }}} \frac{I_{U P}-I_{D N}}{I_{D N}}=2 \pi \frac{\Delta t_{o n}}{T_{r e f}} \frac{\Delta I_{C P}}{I_{D N}} \quad \text { or } \\
& \tau=\Delta t_{\text {on }}\left(I_{U P}-I_{D N}\right) / I_{D N}=\Delta t_{o n} \Delta I_{C P} / I_{D N}
\end{aligned}
$$

Here $I_{\mathrm{UP}}$ is assumed to be larger than $I_{\mathrm{DN}}$. If $I_{\mathrm{DN}}$ is larger than $I_{\mathrm{UP}}$, Equation (3.2) can be expressed as

$$
\begin{aligned}
& \theta_{S P O}=2 \pi \frac{\Delta t_{o n}}{T_{r e f}} \frac{I_{D N}-I_{U P}}{I_{U P}}=2 \pi \frac{\Delta t_{o n}}{T_{r e f}} \frac{\Delta I_{C P}}{I_{U P}} \quad \text { or } \\
& \tau=\Delta t_{o n}\left(I_{D N}-I_{U P}\right) / I_{U P}=\Delta t_{o n} \Delta I_{C P} / I_{U P}
\end{aligned}
$$




\subsection{Concept of the Proposed SPO Reduction Technique}

The conceptual diagram of the proposed SPO reduction technique is shown in Figure 3.3 [19]. Figure 3.3(a) shows the S-domain model of the cyclic reference injection based MDLL. Ideally, in the lock state, the phase error between $\theta_{R}$ and $\theta_{d i v}$ is zero. In reality, the non-ideal effect of charge up will cause the phase error, which is a constant non-zero value in the lock state, also known as SPO. The SPO is modeled as an additive phase error $\theta_{S P O}$ in Figure 3.3 (b). If a gain stage with the gain of $G(\mathrm{G}>1)$ is inserted between the PD/PFD and the charge pump as shown in Figure 3.3(c), and the charge pump current is decreased by a factor of $G$, then the MDLL loop characteristic will not be changed but the equivalent SPO, in the phase domain, will be decreased to,

$$
\theta_{S P O_{-} e q v}=\theta_{S P O} / G
$$

The complete S-domain model of MDLL with the proposed SPO reduction technique is shown in Figure 3.3(d).

Similar to Figure 3.3 (d), a time domain model of MDLL with the proposed SPO reduction technique is shown in Figure 3.4.Similar to equation (3.4), the time domain equivalent SPO can be expressed as,

$$
\tau_{-e q v}=\tau / G
$$




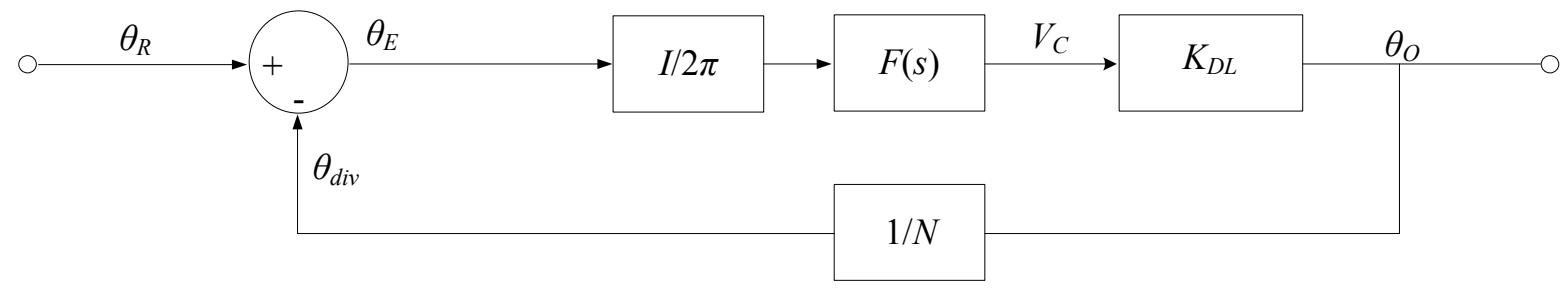

(a)

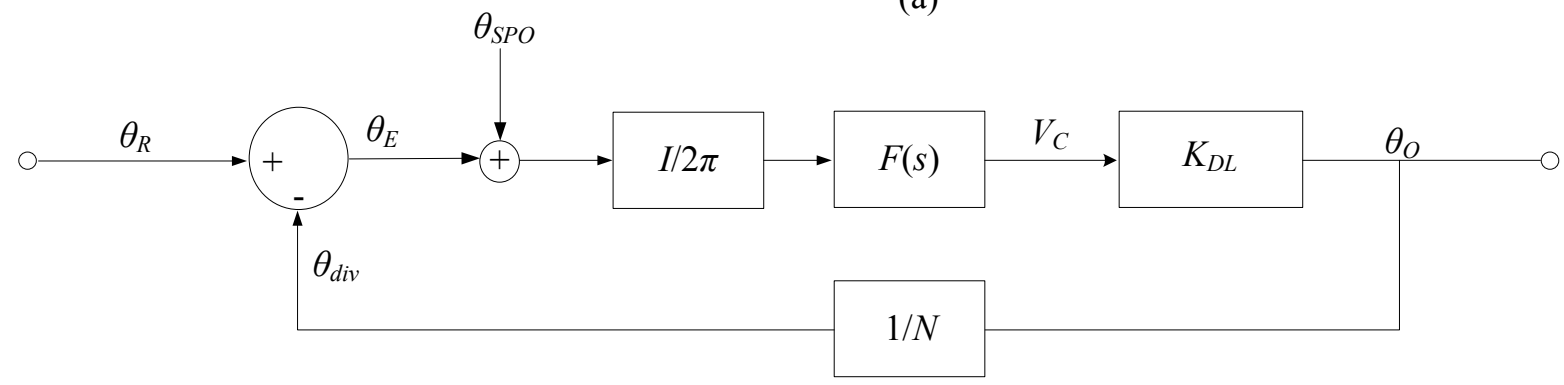

(b)

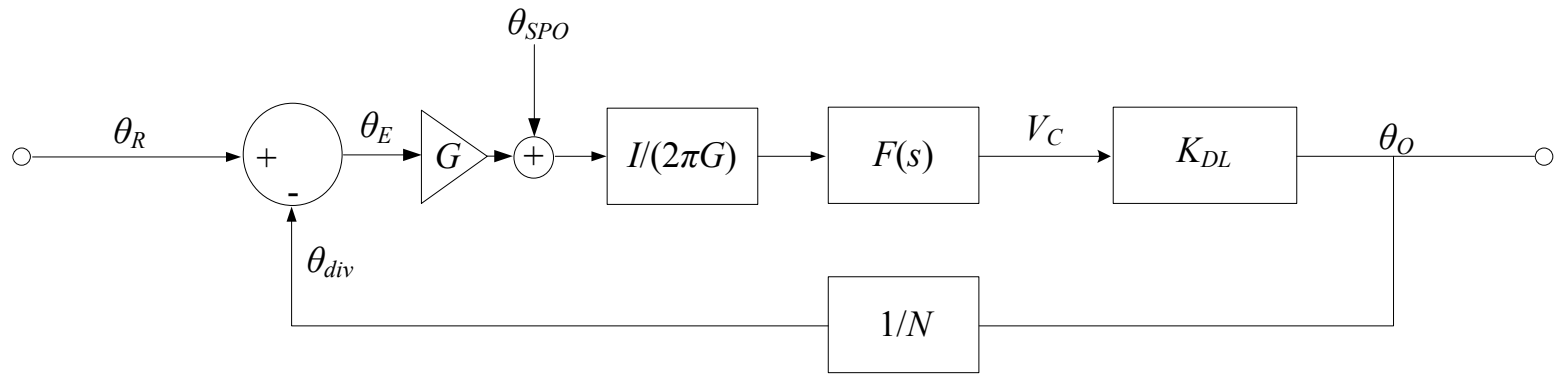

(c)

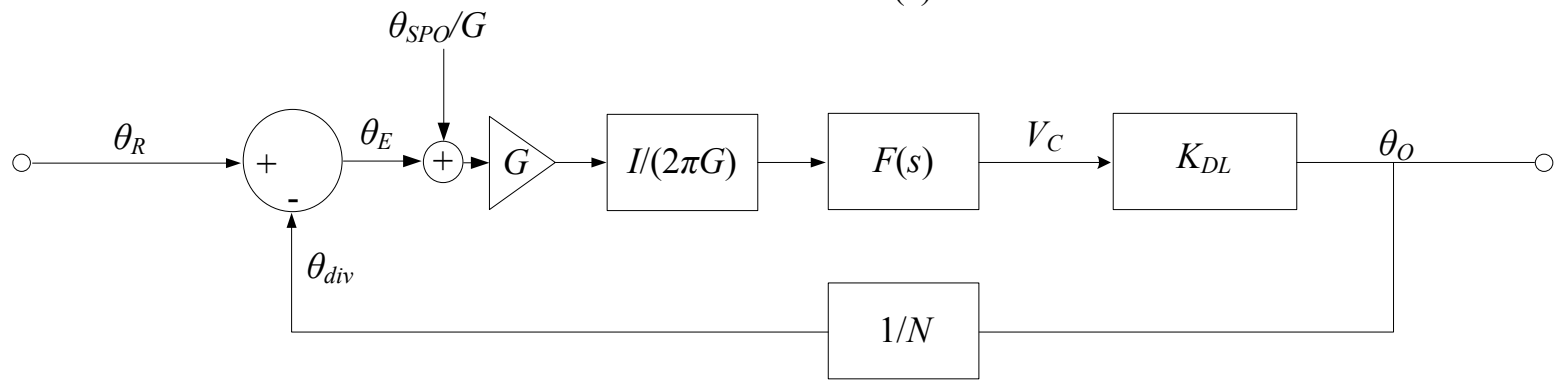

(d)

Figure 3.3: Conceptual diagram of the proposed SPO reduction technique. (a) S-domain model of MDLL; (b) S-domain model of MDLL with SPO $\theta_{S P O}$; (c) S-domain model of MDLL with SPO $\theta_{S P O}$ and a gain stage insertion; (d) Equivalent SPO becoming $\theta_{S P O} / G$ after a gain stage insertion [19]. 


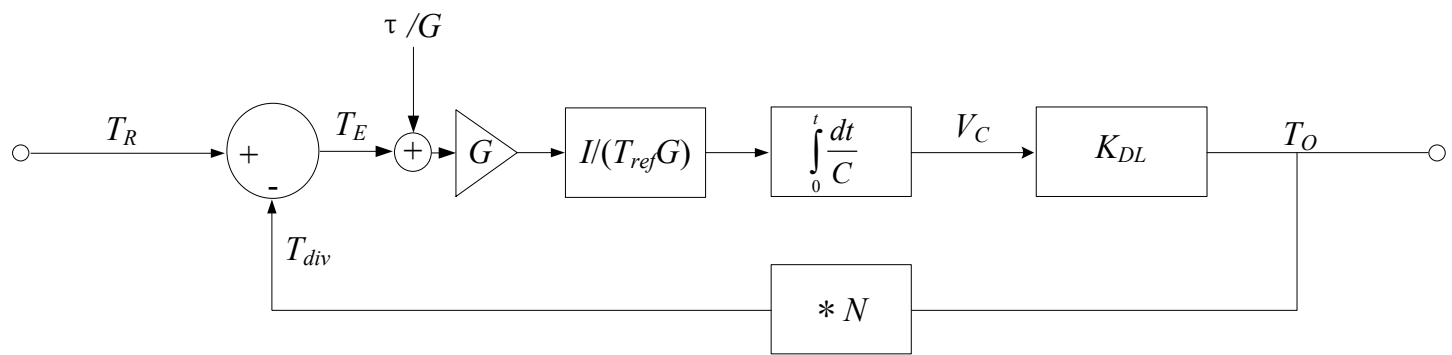

Figure 3.4: The time domain model of MDLL with the proposed SPO reduction technique.

\subsection{Proposed SPO Reduced MDLL}

To achieve a gain stage between the PD/PFD and the charge pump, one possible way is to employ time amplifiers to amplify the up and down signals of the PFD. Another way is to convert the time domain signal, VCDL output phase signal, to a voltage signal because processing a voltage-domain signal is much easier than processing a time-domain one. 


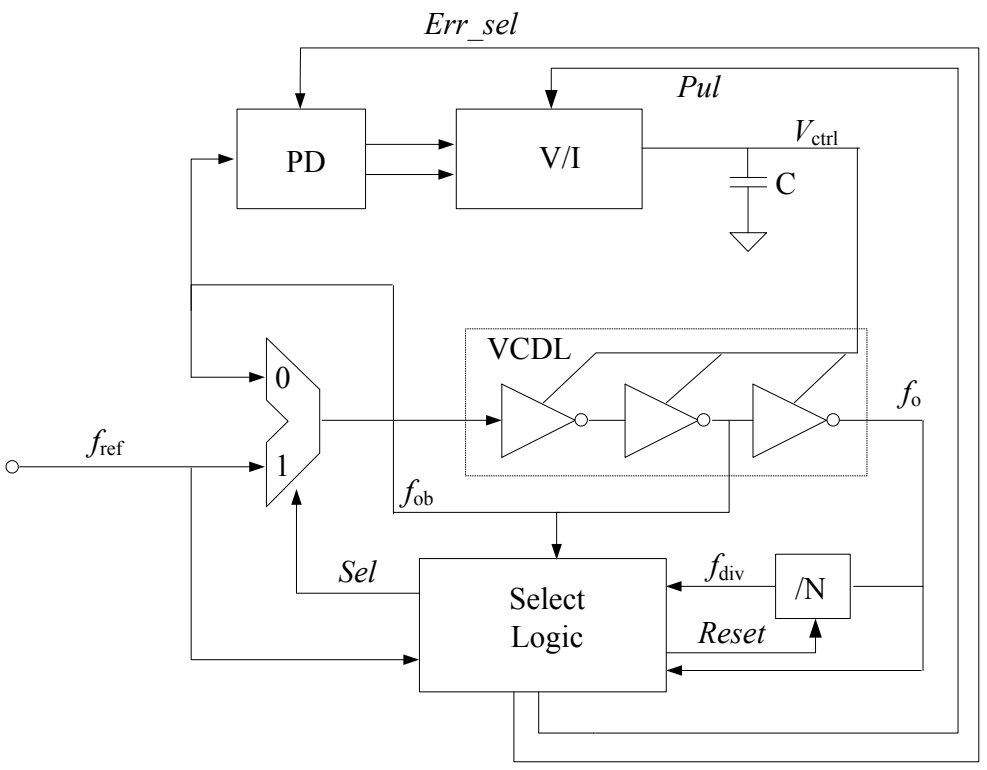

(a)

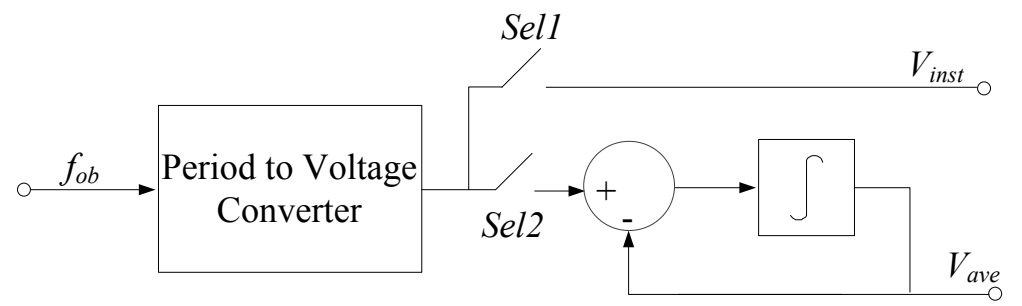

(b)

Figure 3.5: Block diagrams of (a) the proposed MDLL and (b) the PD.

This work adopts the second method. The system level architecture of the proposed MDLL is shown in Figure 3.5(a). It comprises a PD, a voltage to current converter (V/I), a MUX, a divide-by-N divider, a select logic, a VCO-like VCDL and one loop filter (capacitor C). The delay chain is configured as a VCO or a VCDL depending on which setting is chosen in the MUX. That is, when the setting is 1 , the rising edge of $f_{\text {ref }}$ propagates in the delay chain and when the setting is changed to 0 , the rising edge circulates in the delay chain 
which is essentially configured as a VCO. The delay chain output, $f_{\mathrm{o}}$, goes to a divider. After $N$ output cycles, a divided-by-N signal, $f_{\text {div }}$, is generated and goes to the select logic. After four more output cycles, the select logic generates the MUX control signal, sel, and a reset pulse, Reset, which resets the registers in the divider and starts a new divided-by-N cycle. The select logic generates $\mathrm{Pul}$ and Err_sel pulse signals every $N+4$ output cycles to control the PD and V/I blocks.

Compared with a conventional PD, the PD of this MDLL shown in Figure 3.5(b) has only one input which is the output of VCDL. Because this architecture avoids the comparison of two different edge signals, it can significantly reduce the error caused by the path mismatch [15]. The PD consists of a period to voltage converter and a delta modulator. The simplified timing diagram of the PD is shown in Figure 3.6. The first N-1 cycles of the MDLL output waveforms have evenly distributed in-lock error and uniform period but the $N$ th cycle has a either bigger or smaller period due to SPO. The operation principle of the PD is: firstly through a period to voltage converter the $\mathrm{VCDL}$ output $f_{\mathrm{ob}}$ is converted to a voltage pulse-train signal $V_{\text {into }}$, the amplitude of each pulse is proportional to its period, then the converted voltage pulse-train signal of the $N$ th cycle is sampled by Sell to produce $V_{\text {inst. }}$ The converted voltage pulses of the rest N-1 cycles are sampled by Sel2 and go into a delta modulator to generate the period average signal $V_{\text {ave }}$. The delta modulator provides a highly 
accurate average period of the first $\mathrm{N}-1$ cycles of VCDL output. $V_{\text {inst }}$ subtracts $V_{\text {ave }}$ through V/I block to extract SPO information and this information is converted to the VCDL control voltage $V_{\text {ctrl }}$ through the loop filter capacitor $C$. Figure 3.6 shows the case that the $N$ th cycle has a larger period, which means that the amplitude of $V_{\text {inst }}$ is larger than that of $V_{\text {ave }}$. The amplitude of $V_{\text {inst }}$ will be smaller than that of $V_{\text {ave }}$ if the $N$ th cycle has a smaller period.

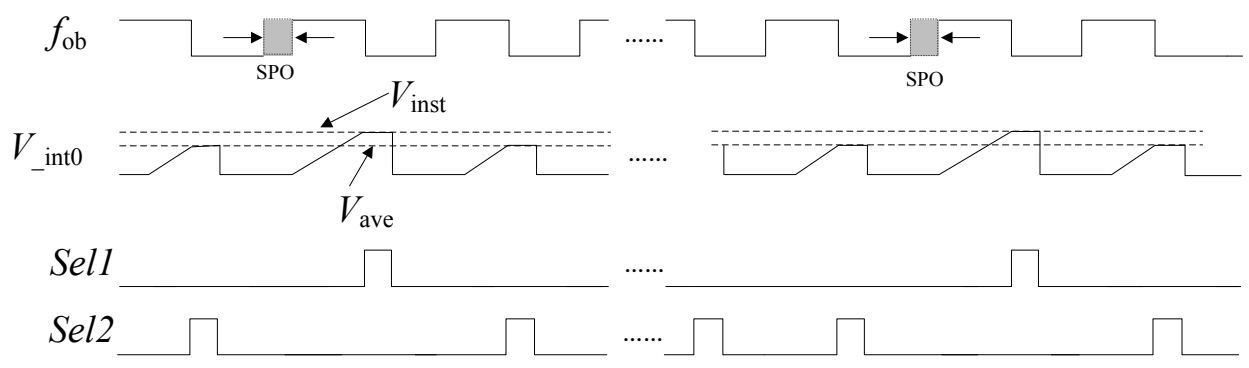

Figure 3.6: Simplified timing diagram of the PD.

\subsection{Simulink Model of Proposed MDLL [19]}

The proposed MDLL described above was verified by a behavioral model built in Simulink as shown in Figure 3.7. The Simulink model is based on the time domain model of the MDLL with the proposed SPO reduction technique as shown in Figure 3.4 and the block diagram of proposed MDLL shown in Figure 3.5(a). It mainly consists of a VCDL modeled with a Variable Delay Line, a divider with division ratio of 10, a block of Extract period value extracting each cycle period value from VCDL output $f_{\text {out }}$ waveform and a block of 
$P D+V / I$ extracting SPO shown in Figure 3.5(a). The loop filter capacitor is modeled with Integrator1. Loop Gain1 is the product of factor of period to voltage conversion, factor of voltage to current conversion, loop filter gain and VCDL gain. $T n$ in Figure 3.8 is the $n$-th output period, Gain1, the gain of period to voltage converter, is equivalent to the gain of the gain stage, $G$, in Figure 3.3. Gain ${ }^{*}$ Gain2 is equivalent to the gain of the integrator in Figure 3.5(b). The $N$ th period is deducted by the integrated value to extract the period error value which is integrated to generate a new average period value. This procedure will be repeated as long as the circuit operates. Since the main in-lock error appears in the last period within one reference cycle, this error detection is controlled by Sell. Then the error value is sent to the loop filter to generate a control voltage to tune the VCDL delay. Non-idealities causing SPO are modeled as an additive constant which is labeled as "SPO" shown in Figure 3.8. This "SPO" has the unit of time. 


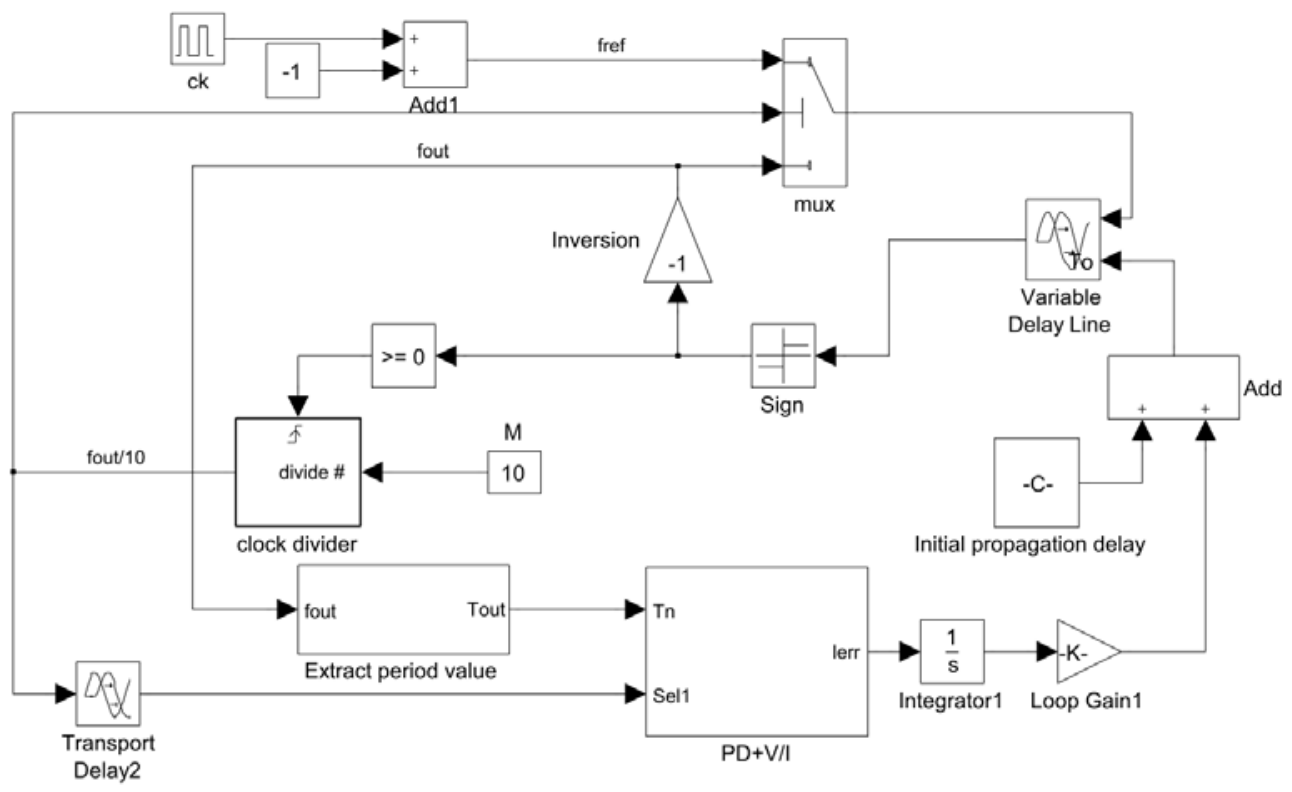

Figure 3.7: Simulink model of the proposed MDLL.

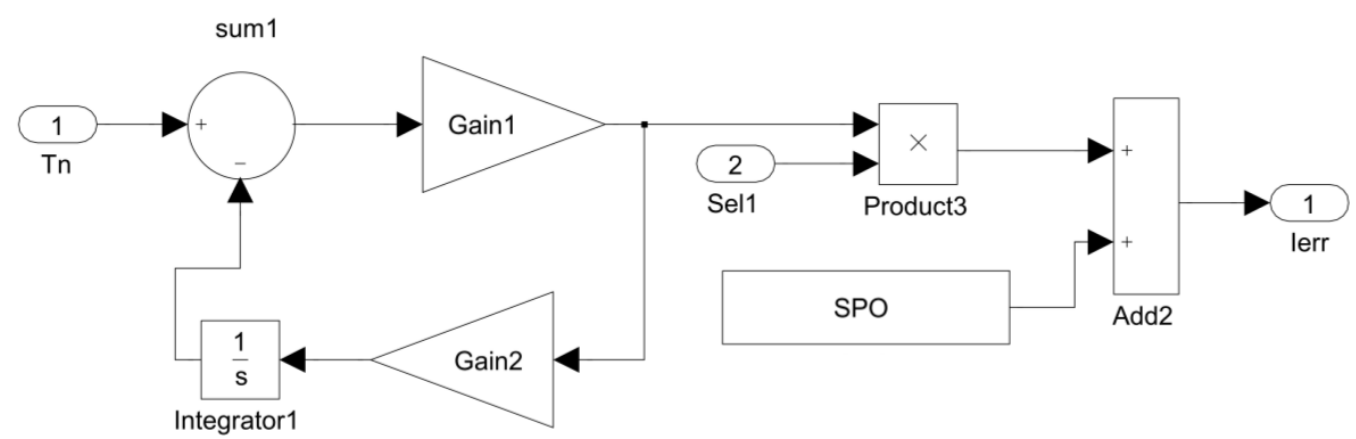

Figure 3.8: Model of PD + V/I.

Because both DDR3 and DDR4 are main stream applications nowadays and both can operate at $800 \mathrm{MHz}$ and higher clock, the operation frequency of the proposed Simulink model of MDLL is set to $1 \mathrm{GHz}$ with a reference signal $f_{\text {ref }}$ of $100 \mathrm{MHz}$ and a division ratio of 
10. The Initial propagation delay in Figure 3.7 is $480 \mathrm{ps}$ which is smaller than 500ps in locked state because usually an MDLL loop has a starter circuit which set the minimum propagation delay at the initial condition; the Loop Gain1 is $1.28 \mathrm{e} 4$ and Transport Delay2 is 2.5ns. Simulation results of the MDLL output signal spectra are shown in Figure 3.9. The center frequencies in all of the spectra plots of Figure 3.9 are $1 \mathrm{GHz}$. In Figure 3.9(a) (to make the plot more readable, the center frequency has been shifted to 0 ), the time domain SPO $\tau$ and Gain1 are set to 0 and 1 respectively, the spectrum plot shows that the reference spur has similar power to the noise floor. In Figure 3.9(b), $\tau=2 \mathrm{e}-11 \mathrm{ps}$ and $\operatorname{Gain} 1=1$, the reference spur $\approx-34 \mathrm{dBc}$. In Figure 3.9(c), $\tau=2 \mathrm{e}-11 \mathrm{ps}$ and Gain $1=10$, the reference spur $\approx$ $-54 \mathrm{dBc}$. In Figure 3.9(d), $\tau=2 \mathrm{e}-11 \mathrm{ps}$ and Gain $1=50$, the reference spur $\approx-68 \mathrm{dBc}$. The results are summarized in Table 3.1.

Table 3.1 Simulation results of the Simulink model of the MDLL

\begin{tabular}{|c|c|c|c|c|}
\hline$\tau$ (time domain SPO) & 0 & $20 \mathrm{ps}$ & 20ps & $20 \mathrm{ps}$ \\
\hline $\begin{array}{l}\text { Gain1 (gain of the } \\
\text { insertion gain stage) }\end{array}$ & 1 & 1 & 10 & 50 \\
\hline Spur (simulated) (dBc) & Close to the noise floor & -34 & -54 & -68 \\
\hline $\begin{array}{l}\text { Spur (calculated from } \\
(3.7))(\mathrm{dBc})\end{array}$ & $-\infty$ & -34 & -54 & -68 \\
\hline $\begin{array}{c}\tau_{-e q v}(\text { Calculated from } \\
(3.5))\end{array}$ & 0 & $20 \mathrm{ps}$ & $2 \mathrm{ps}$ & $0.4 \mathrm{ps}$ \\
\hline$\tau_{-e q v}($ Calculated from & 0 & $20 \mathrm{ps}$ & $2 \mathrm{ps}$ & $0.4 \mathrm{ps}$ \\
\hline
\end{tabular}




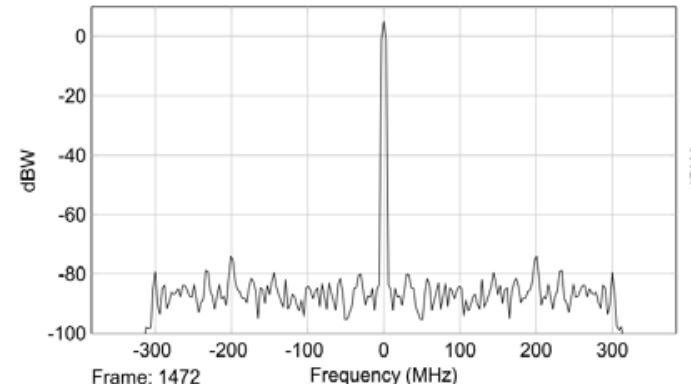

(a)

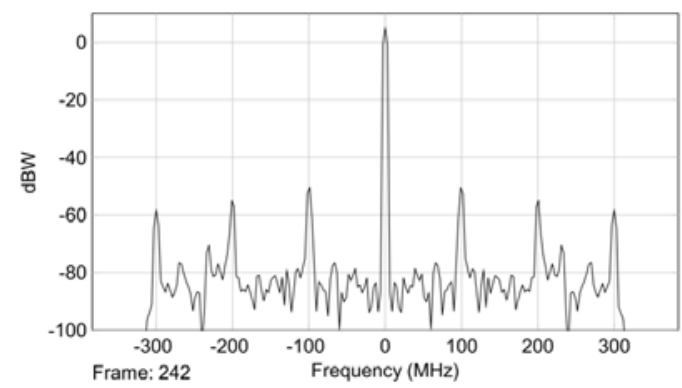

(c)

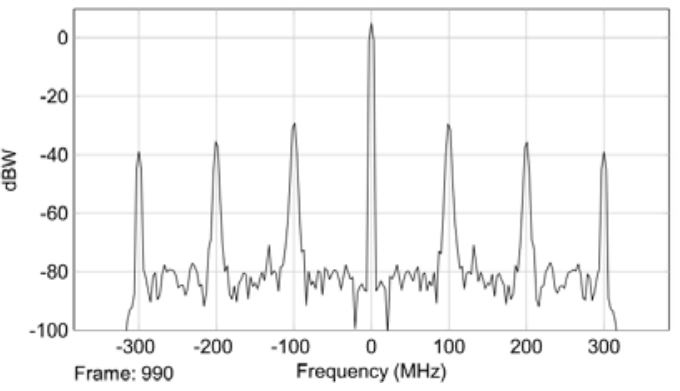

(b)

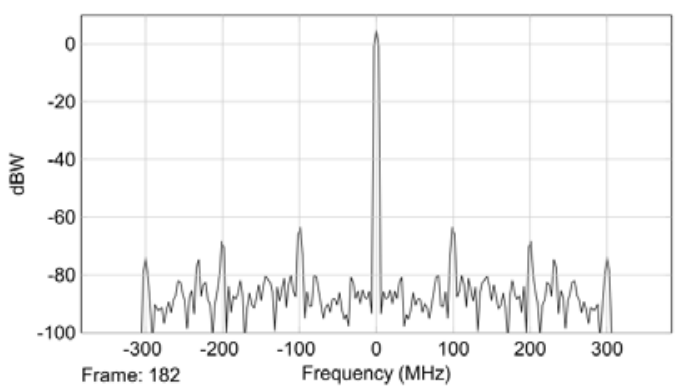

(d)

Figure 3.9: Spectral plots of VCDL output under different conditions. (a) SPO=0, Gain 1=1; (b) $\mathrm{SPO}=2 \mathrm{e}-11 \mathrm{ps}$, Gain1=1; (c) SPO=2e-11ps, Gain1=10; (d) SPO=2e-11ps, Gain1=50.

The time domain SPO and the reference spur value can be expressed by the following equation [15],

$$
\tau_{- \text {eqv }} \approx T_{\text {out }} \times 10^{\operatorname{spur}(d B c) / 20}
$$

where $\tau$ is the time domain SPO, $T_{\text {out }}$ is the MDLL ideal output period and spur is the $\mathrm{dB}$ value of the reference spur.

Plug equation (3.5) into (3.6) and the spur can be expressed as, 


$$
\operatorname{spur}=20 \log \left(\tau_{-e q v} / T_{\text {out }}\right)=20 \log \left((\tau / G) / T_{\text {out }}\right)
$$

The calculated and simulated spur values vs $G$, the gain of the insertion gain stage, are plotted in Figure 3.10. The spur value calculated from equation (3.7) is plotted with a solid line and the simulated spur values as shown in Figure 3.9 with randomly chosen $\tau$, the time domain SPO, and randomly chosen $G$, the gain of the insertion gain stage, are marked with "o".

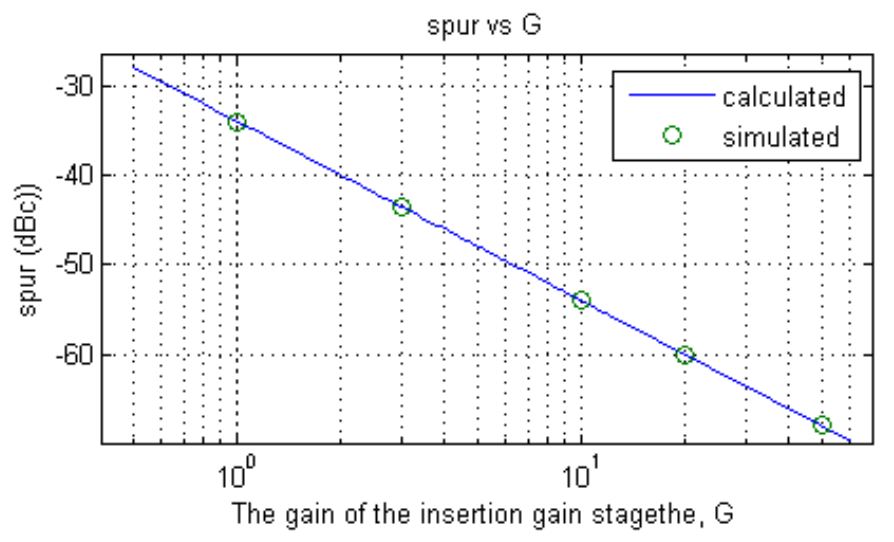

Figure 3.10: spur values vs the gain $(G)$ of the insertion gain stage.

From Table 3.1 and Figure 3.10, one can see that the equivalent SPO is inversely proportional to the gain of the insertion gain stage, which verifies the efficiency of equation (3.5), namely, the proposed SPO reduction technique.

\subsection{Simulation of the Proposed MDLL at the Transistor Level}

The system level of the proposed MDLL is shown in Figure 3.5(a) [20]. 


\subsubsection{Select Logic}

The select logic (SL) shown in Figure 3.11 works as a timing control unit to synchronize the operation of the PD, the multiplexer, the divider and the voltage to current converter $(\mathrm{V} / \mathrm{I})$. DFF1 and DFF2 form a count-to-2 circuit and the output of DFF2, $Q 2$, goes high at the second rising edge of $f_{\text {div }}$. The operation after the second rising edge of $f_{\text {div }}$ is illustrated in Figure 3.12. Signal $\mathrm{Sel}$ is obtained by sampling $Q 2$ at the rising edge of $f_{\mathrm{ob}}$. Reset is generated at the rising edge of $f_{\text {ref. }}$ The Err_sel signal is generated by sampling Q3 at the rising edge of $f_{\mathrm{o}}$; it is a positive pulse with its width as one output period. The signal Pul is generated at the falling edge of $f_{\text {ref }}$ through an $A N D$ gate with a delay buffer.

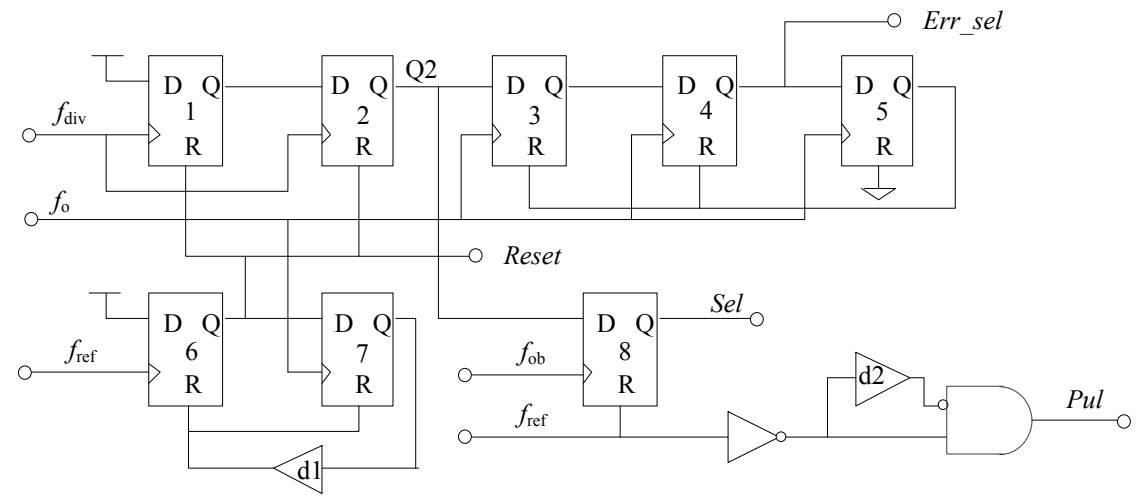

Figure 3.11: Circuit of the SL [20]. 


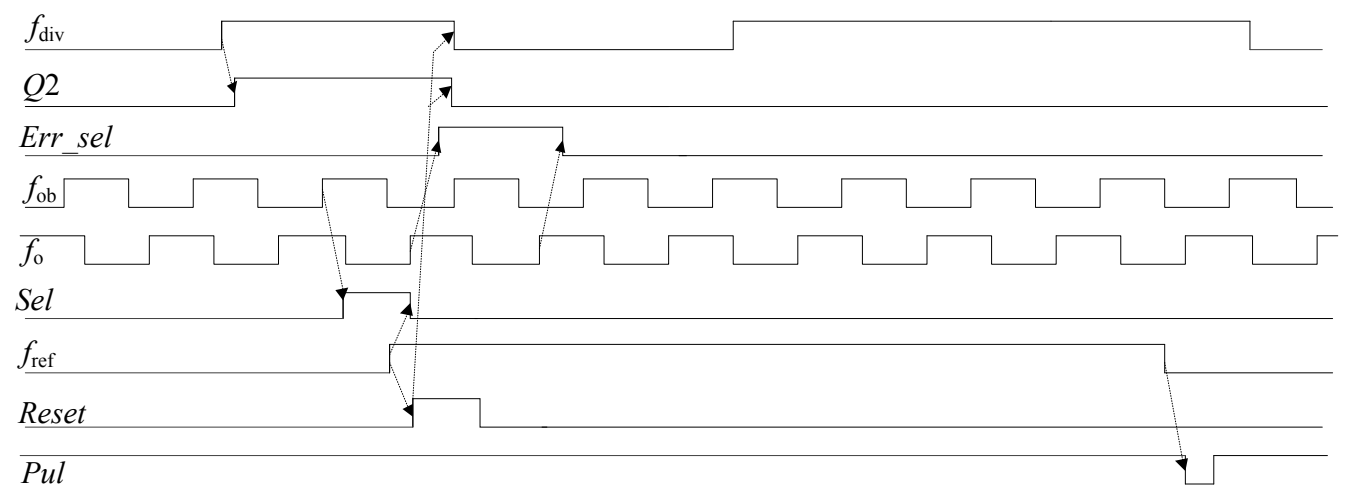

Figure 3.12: Timing diagram of the SL [20].

\subsubsection{Phase Detector}

The block diagram of the PD is shown in Figure 3.5(b). First, the instantaneous period of VCDL output, $f_{\text {ob }}$, is converted into a voltage pulse-train signal $V_{-}$into, and then $V_{-}$into goes to a delta modulator (DM) to generate a voltage signal of the average period of the signal $f_{\mathrm{ob}}$.

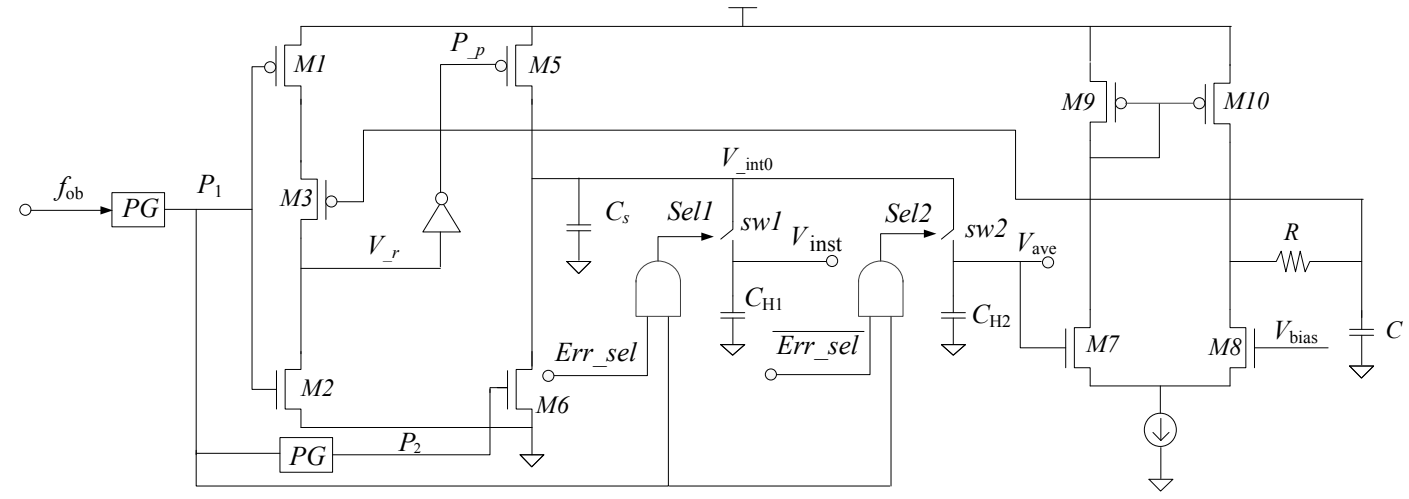

Figure 3.13: Schematic of the PD.

The schematic and timing diagram of the PD are respectively shown in Figure 3.13 and Figure 3.14. Similar to the error detector in [13], a narrow pulse $P_{1}$ is generated by the 
pulse generator (PG) at each falling edge of $f_{\mathrm{ob}}$, and its pulse width increases by an amount corresponding to the average period duration to generate $P \_$p. $P \_p$ is inverted and integrated by a high-gain integrator. The integrated result $V_{\text {into }}$ is sampled by Sell and Sel2 to generate $V_{\text {inst }}$ and $V_{\text {ave }} . V_{\text {int0 }}$ is reset by $P_{2}$ through $M 6$ before the next pulse of $P_{1}$ arrives. $V_{\text {ave }}$ is compared with a predefined value, $V_{\text {bias, }}$ and the comparison result is used to adjust the incremental value of the pulse width of $P_{1}$ accordingly. The bias voltage $V_{\text {bias, }}$, which determines the $V_{\text {ave }}$, is set to be approximately half of the supply voltage $V_{\mathrm{DD}}$ to ensure that the integrator, capacitor $C_{S}$, works in its high-gain region. So a high static phase offset resolution can be achieved. The internal loop can also ensure that the PD operates at the highest speed and the following stage, a differential pair, has a higher input sensitivity. During one cycle of the $P G$ circuit shown in Figure 3.15, when the falling edge of the input signal samples the high level supply voltage, the output $\mathrm{Q}$ turns to a high level .Then the high-level Q feeds back to the reset $\mathrm{R}$ and is reset to a low level. The $P G$ repeats the same operation in another cycle. 


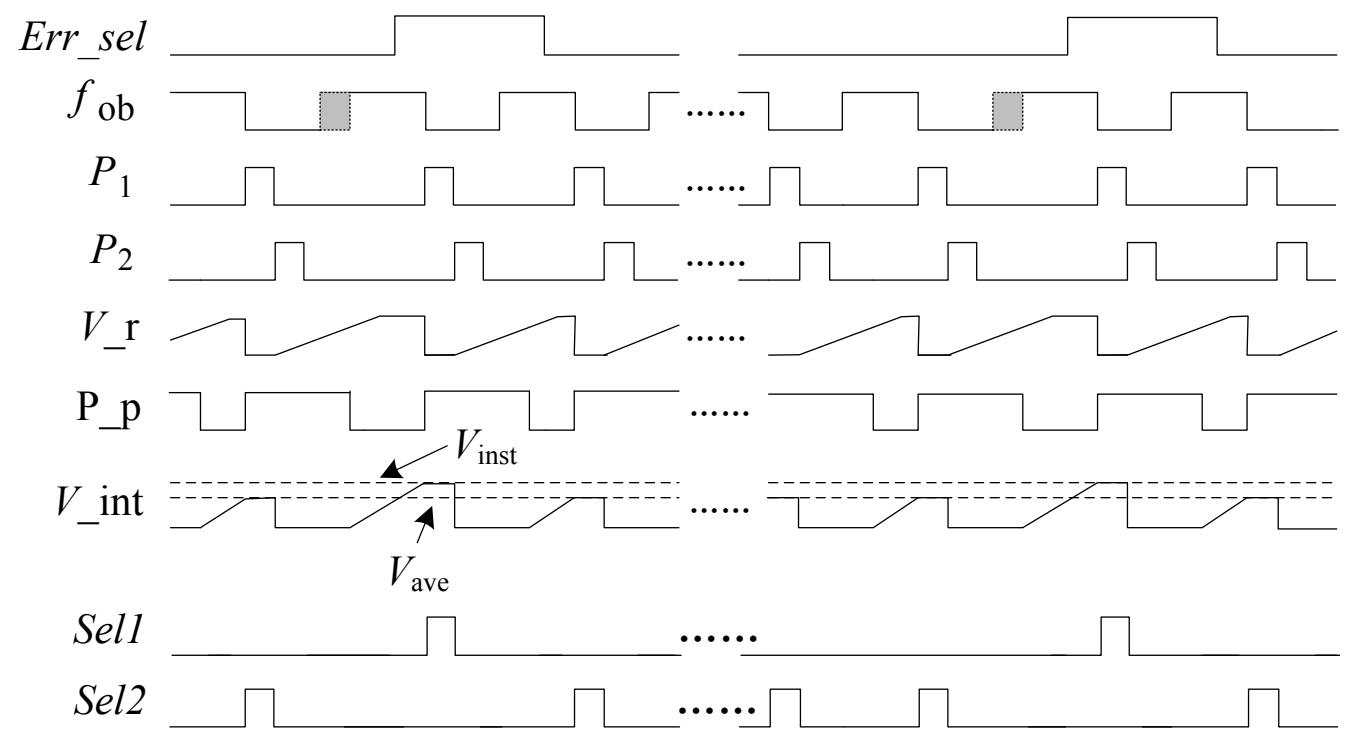

Figure 3.14: Timing diagram of the PD.

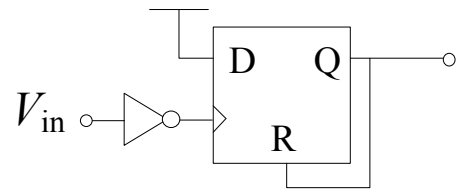

Figure 3.15: The PG circuit.

The transistors $M 5$ and capacitor $C_{\mathrm{S}}$ in Figure 3.13 form an equivalent $\mathrm{RC}$ charging circuit as shown in Figure 3.16. M5 can be modeled as a switch in series with a resistor which is $R_{o n}$ of M5. The charge time is the negative pulse width of each cycle of signal $P_{p}$. So $V_{-}$into can be expressed as

$$
V_{-} \text {int } 0, i=V_{D D}\left(1-e^{-\tau_{i} / R_{o n} C_{S}}\right)
$$


where $i$ is the $i$-th cycle of $P_{p}, \tau_{\mathrm{i}}$ is the negative pulse width of the $i$-th cycle of $P_{p}$ and $R_{o n}=\frac{1}{\mu_{n} C_{o x} \frac{W}{L}\left(V_{G S}-V_{T H}\right)}$ [55]. In the stable locked state, $V_{-i n t 0}=V_{\text {bias }}=V_{\mathrm{DD}} / 2$, so the $i$-th cycle negative pulse width $\tau_{\mathrm{i}}$ can be calculated as,

$$
\tau_{i}=R_{o n} C_{S} \ln 2
$$

From equation (3.9) one can see that in the stable locked state, the negative pulse width $\tau$ of each cycle is equal to a constant. After $\tau_{\mathrm{i}}$ is determined, the size of $M 5$ can be obtained, the ramp-up time of $V_{-}$can be obtained, then the size of $M 3$ can be optimized.
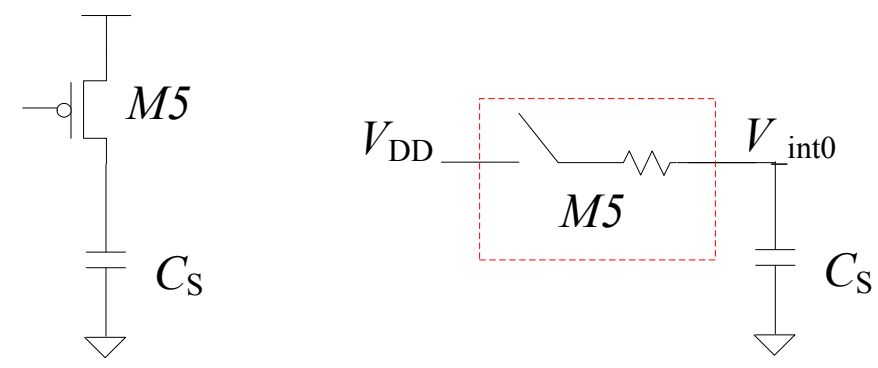

Figure 3.16: The circuit of series M5 and CS and their equivalent RC charging circuit..

$M 6$ and $C_{\mathrm{S}}$ form an equivalent $\mathrm{RC}$ discharging circuit. In order to achieve a shorter discharge time, the size of $M 6$ can be relatively large. Switches sw1 and sw2 are transmission gates with the same size for both PMOS and NMOS transistors to cancel the charge injection effect [56]. Sizes and values of the devices are summarized in Table 3.2. 
Table 3.2 Transistors and capacitors used in the circuit of PD.

\begin{tabular}{|c|c|c|c|}
\hline Device & $\mathrm{W} / \mathrm{L}(\mu \mathrm{m} / \mu \mathrm{m})$ & Device & $\mathrm{W} / \mathrm{L}(\mu \mathrm{m} / \mu \mathrm{m})$ or value \\
\hline M1 & $25 / 0.2$ & PMOS for sw1 & $5 / 0.2$ \\
\hline M2 & $8 / 0.2$ & NMOS for sw1 & $5 / 0.2$ \\
\hline M3 & $20 / 0.2$ & PMOS for sw2 & $5 / 0.2$ \\
\hline M5 & $7 / 0.2$ & NMOS for sw2 & $5 / 0.2$ \\
\hline M6 & $5 / 0.2$ & $\mathrm{C}_{\mathrm{S}}$ & $0.1 \mathrm{pF}$ \\
\hline M7, M8 & $4 / 1$ & $\mathrm{C}_{\mathrm{H} 1}, \mathrm{C}_{\mathrm{H} 2}$ & $1 \mathrm{pF}$ \\
\hline M9,M10 & $5 / 1$ & & \\
\hline
\end{tabular}

\subsubsection{Voltage to Current Converter (V/I)}

The schematic of V/I [57] is shown in Figure 3.17. The voltages $V_{\text {inst }}$ and $V_{\text {ave }}$ are converted into currents by $M 1$ and $M 2$. The currents are then mirrored by $M 5$ and $M 9$ respectively. $M 9$ sources the charge pump. The current mirrored by M5 flows into diode connected M7. M7 and M8 consist of a current mirror and M8 sinks the charge pump. The current mirrored by M9 injects either into the loop filter $C$ or into a dumping node $V_{\text {dum }}$, depending on the state of the signal Pul. The diode connected transistor $M 6$ is added to improve the drain node voltage matching of the current mirror transistor M4 and M5. When $V_{\text {inst }}$ is larger than $V_{\text {ave }}$, M8 sinks more current than M9 sources one, Vctrl will decrease. When $V_{\text {inst }}$ is smaller than $V_{\text {ave, }}$ M8 sinks less current than the current which M9 sources and Vctrl will increase. When $V_{\text {inst }}$ is equal to $V_{\text {ave, }}$, the sink and source currents are the same, Vctrl will maintain the same value. 
$I_{\text {bias }}$ is $100 \mu \mathrm{A}$. The current source switches use near minimum size and the dumping capacitor is set to $1 \mathrm{pF}$, to reduce the effect of clock feed-through and charge injection [57]. The $P u l$ is a pulse signal generated by select logic shown in Figure 3.11. When the Pul is high, $\overline{P u l}$ is low, the current sources $M_{s w p}$ and $M_{s w n}$ are switched on simultaneously, $M 9$ and M8 are connected and the current will flow into or flow out of the loop filter. When the Pul is low, $\overline{P u l}$ is high. The current sources are steered away to $V_{\text {dum }}$ instead of being switched off to alleviate the charge sharing between the loop filter and the current sources [58]. The device sizes and values in Figure 3.17 are summarized in Table 3.3.

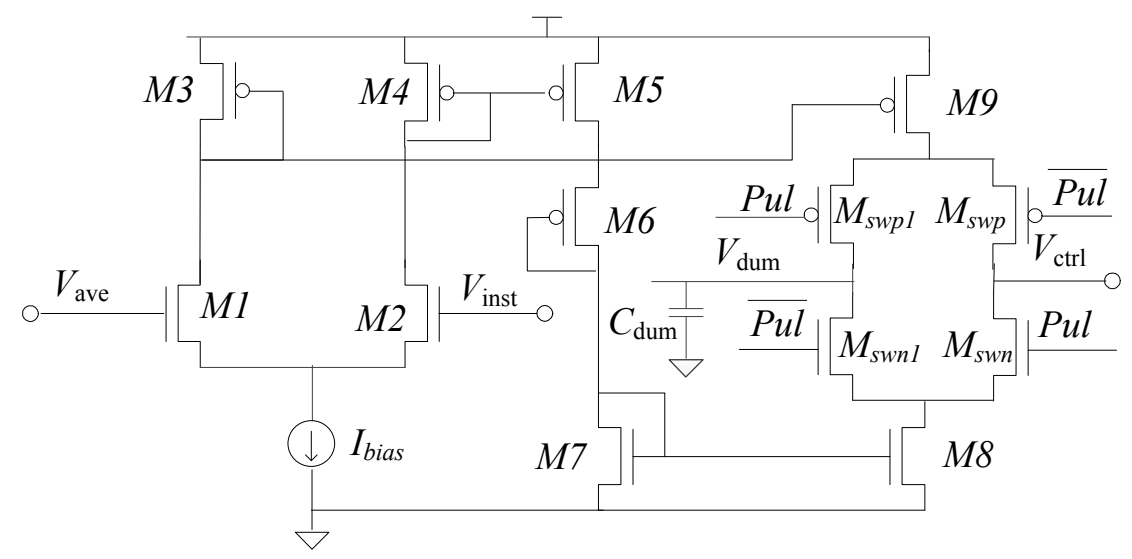

Figure 3.17: Schematic of V/I. 
Table 3.3 Transistors and capacitors used in the circuit of V/I.

\begin{tabular}{|c|c|c|c|}
\hline Device & $\mathrm{W} / \mathrm{L}(\mu \mathrm{m} / \mu \mathrm{m})$ & Device & $\mathrm{W} / \mathrm{L}(\mu \mathrm{m} / \mu \mathrm{m})$ or value \\
\hline$M 1, \mathrm{M} 2$ & $4 / 1$ & $M_{\text {swn, }} M_{\text {swn } 1}$ & $0.3 / 0.2$ \\
\hline$M 3, \mathrm{M} 4, \mathrm{M} 5, \mathrm{M} 9$ & $20 / 1$ & $M_{\text {swp, }} M_{\text {swpl }}$ & $0.3 / 0.2$ \\
\hline$M 6$ & $10 / 1$ & $C_{\text {dum }}$ & $1 \mathrm{pF}$ \\
\hline$M 7, M 8$ & $7.5 / 1$ & & \\
\hline
\end{tabular}

\subsubsection{VCDL and its Bias Generation Circuit}

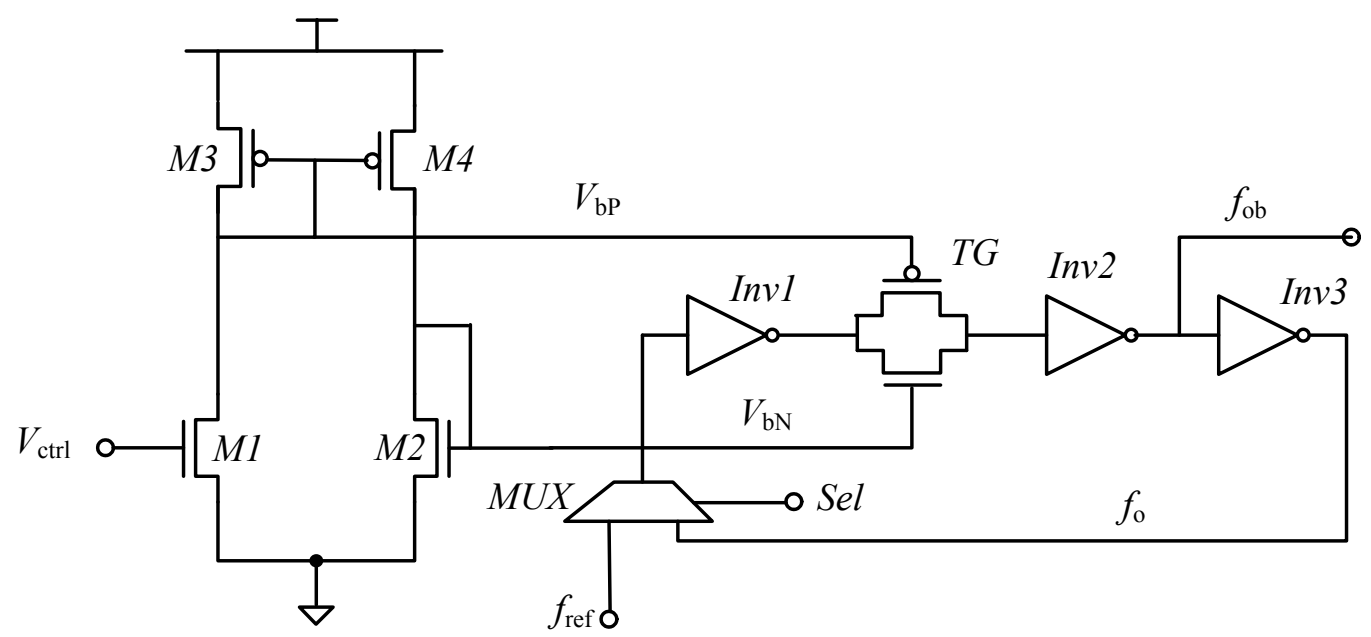

Figure 3.18: The VCDL and its bias generation circuit.

As shown in Figure 3.18, the VCDL and its bias generation circuit consists of a $M U X$, three same sized inverters and a voltage controlled transmission gate. When the MUX selection signal $\mathrm{Sel}$ is 1 , the clean edge of $f_{\text {ref }}$ propagates in and when the $\mathrm{Sel}$ is 0 , the VCDL operates 
as a VCO. The transmission gate $T G$ is controlled by the complementary bias voltages $V_{\mathrm{bP}}$ and $V_{\mathrm{bN}}$. The VCDL control voltage $V_{\mathrm{ctrl}}$ is converted into the current through a common source amplifier $M 1$, then the bias voltage $V_{b P}$ is generated through a diode connected PMOS transistor M3. M3 and M4 form a current mirror. The mirrored current through a diode connected NMOS transistor $M 2$ generates the bias voltage $V_{\mathrm{bN}}$. The VCO-like VCDL employs the architecture similar to a transmission-gate based voltage controlled ring oscillator which has a wide tuning range and fast voltage swing [59].

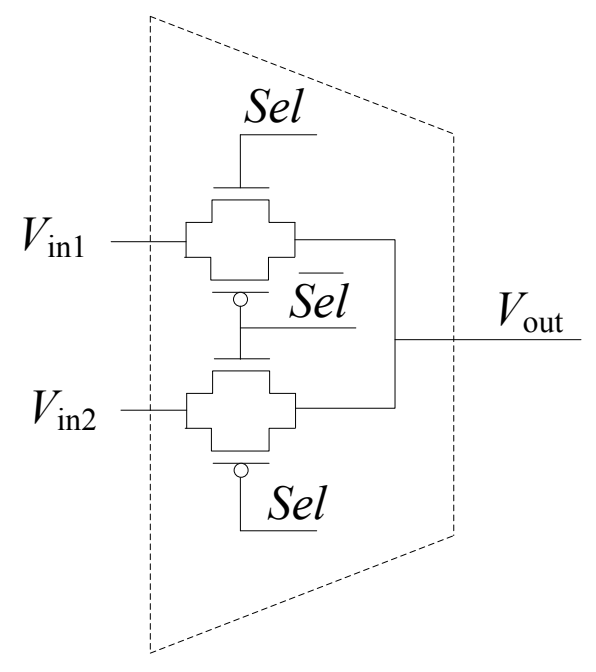

Figure 3.19: The schematic of MUX circuit.

The schematic of the MUX circuit, shown in Figure 3.19, consists of two transmission gates that are controlled by the $\mathrm{Sel}$ and its complementary signal $\overline{\mathrm{Sel}}$. A transmission gate circuit and its equivalent small signal RC model are shown in Figure 3.20. From [60], the effective resistance $\mathrm{R}_{\mathrm{tg}}$ is given in the following equations of (3.10) and (3.11) (these two 
equations appear as (2a) and (2b) in [59]), where the $\alpha$-power law is applied).

$$
\begin{gathered}
R_{t g}=k\left(\frac{\left(1-e^{-1}\right) V_{D D}}{V_{C}-V_{t h}}-0.3\right) \text { for } V_{t h}<V_{C} \leq\left(1-e^{-1}\right) V_{D D}+V_{t h} \\
R_{t g}=k \ln \left(\frac{1}{1-0.5\left(1-e^{-1}\right) \frac{V_{D D}}{V_{C}-V_{t h}}}\right) \text { for }\left(1-e^{-1}\right) V_{D D}+V_{t h}<V_{C}
\end{gathered}
$$

where $k$ is a constant and equal to $\left(V_{D D^{-}} V\right.$ th $) / I_{\mathrm{DO}}, I_{\mathrm{DO}}$ is the current at $\mathrm{VGS}=\mathrm{VDS}=\mathrm{V}_{\mathrm{DD}}, V_{\mathrm{C}}$ is the control voltage at the gate of NMOS transistor of the transmission gate and $V_{\text {th }}$ is the threshold voltage for both transistors.

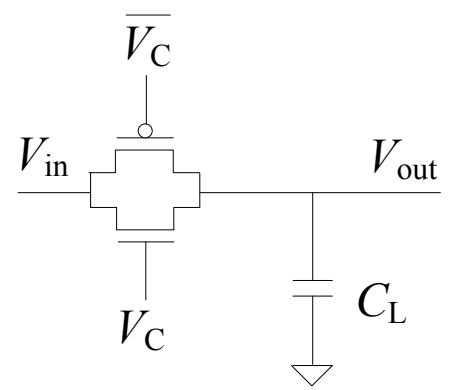

(a)

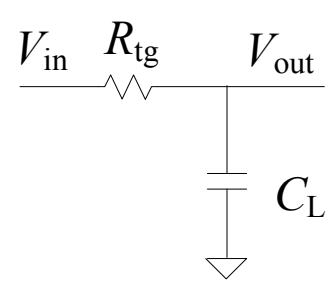

(b)

Figure 3.20: Transmission gate. (a) schematic; (b) equivalent small signal RC model.

From [61], the delay element comprising an inverter and a transmission gate is shown in Figure 3.21(a). Its equivalent RC model is shown in Figure 3.21(b), where $G_{M}$ is the transconductance of a single inverter. Similarly, a single stage inverter delay element can also be modeled with a small signal RC model which is shown in Figure 3.22. 


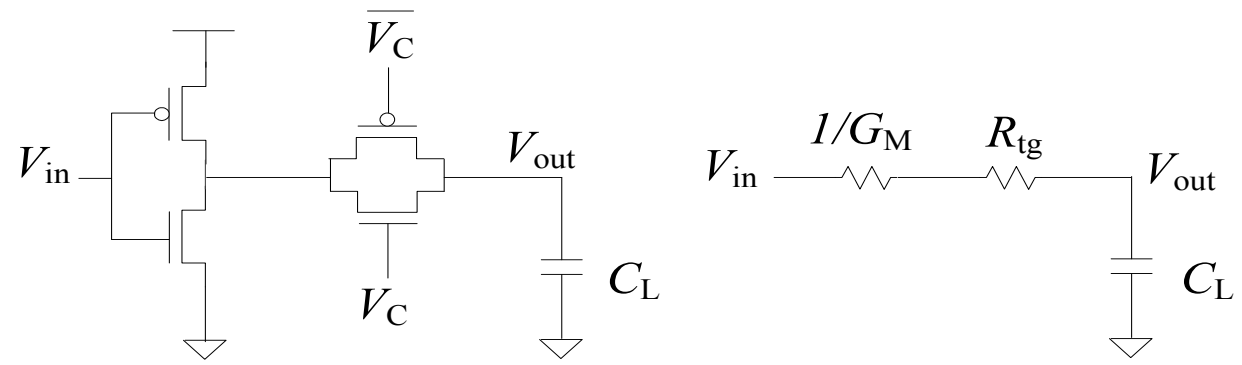

Figure 3.21: The delay element comprising an inverter and a transmission gate. (a) schematic; (b) equivalent RC model.
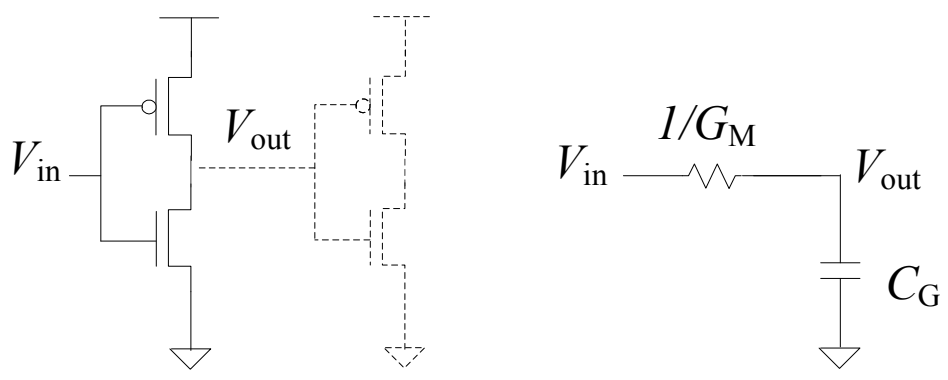

Figure 3.22: Single inverter delay element. (a) schematic; (b) equivalent RC model.

According to the above equivalent RC models of delay elements the MDLL operation frequency can be expressed as,

$$
f_{M D L L}=\frac{1}{2\left(\left(\frac{1}{G_{M}}+R_{t g_{-} m u x}\right) C_{L 1}+\left(\frac{1}{G_{M}}+R_{t g_{-} c t r l}\right) C_{L 2}+\frac{C_{L 3}}{G_{M}}\right)}
$$

where $C_{\mathrm{L} 1}, C_{\mathrm{L} 2}$, and $C_{\mathrm{L} 3}$ are the capacitance at the input nodes of the inverters Inv1, Inv2 and Inv3 respectively. Because the gate-source and gate-drain capacitance of the 
transistors of each inverter accounts for the largest percentage of the total capacitance at that node, in order to simplify the analysis, $C_{\mathrm{L} 1}, C_{\mathrm{L} 2}$, and $C_{\mathrm{L} 3}$ are assumed to be equal and the value is $C_{\mathrm{G}}$. Then equation (3.12) can be simplified to (3.13). $R_{\mathrm{tg} \_ \text {mux }}$ is the equivalent constant resistance of transmission gates in MUX which is controlled by Sel that is either $V D D$ or $V S S . R_{\mathrm{tg} \_ \text {ctrl }}$ is the equivalent resistance of $T G$ which is controlled by a pair of variable bias voltage, $V_{\mathrm{bP}}$ and $V_{\mathrm{bN}}$. So the $R_{\mathrm{tg} \_ \text {ctrl }}$ is an equivalent variable resistance which is used to control the delay of VCDL to make it operate at the destination frequency.

$$
\begin{aligned}
f_{M D L L} & =\frac{1}{2\left(\left(\frac{1}{G_{M}}+R_{t_{g_{-}} \text {mux }}\right) C_{G}+\left(\frac{1}{G_{M}}+R_{t_{-} \_t r l}\right) C_{G}+\frac{C_{G}}{G_{M}}\right)} \\
& =\frac{G_{M}}{2 C_{G}\left(3+R_{t g_{-} m u x} G_{M}+R_{t g_{-} c t r l} G_{M}\right)}
\end{aligned}
$$

When $R_{\mathrm{tg} \_ \text {mux }}$ and $R_{\mathrm{tg} \_ \text {ctrl }}$ are very small and satisfy $\left(R_{t g_{-} m u x}+R_{t g_{-} c t r l}\right) G_{M}<<1$, the VCDL operation frequency will become

$$
f_{M D L L}=\frac{G_{M}}{6 C_{G}}
$$

When $R_{\mathrm{tg} \_ \text {mux }}$ and $R_{\mathrm{tg} \_ \text {ctrl }}$ are very large and satisfy $R_{t g_{-} c t r l} \gg>3+R_{t g_{-} m u x} G_{M}$, equation (3.13) will become,

$$
f_{M D L L}=\frac{1}{2 C_{G} R_{t g_{-} c t r l}}=\left(\frac{1}{2 C_{G} k\left(\frac{\left(1-e^{-1}\right) V_{D D}}{V_{C}-V_{t h}}-0.3\right)}\right)
$$


where $R_{t g \_}$ctrl is replaced by equation (3.10).

A diode connected MOSFET operates as a small signal resistor and the resistance is approximately equal to $1 / \mathrm{g}_{\mathrm{m}}[55]$ when looking into the source of the device, where $\mathrm{g}_{\mathrm{m}}$ is the small signal tranconductance of the MOSFET. So the bias voltage $V_{\mathrm{bP}}$ can be expressed as,

$$
V_{b P}=V_{D D}-g_{m, M 1}\left(V_{c t r l}-V_{t h}\right) / g_{m, M 3}
$$

where $g_{m, M l}$ and $g_{m, M 3}$ are the small signal transconductance of M1 and M3, $V_{D D}$ is the power supply voltage and $V_{\text {ctrl }}$ is the control voltage from the loop filter. Because $M 3$ and $M 4$ consist of a current mirror, the bias voltage $V_{\mathrm{bP}}$ can be approximately expressed as,

$$
V_{b N}=n\left(g_{m, M 1}\left(V_{c t r l}-V_{t h}\right) / g_{m, M 4}\right)
$$

where $n$ is the ratio of current mirror, $g_{m, M 4}$ is the small signal transconductance of $M 4$. If the transistors $M 1, M 2, M 3$ and $M 4$ are sized to satisfy the following condition,

$$
n=g_{m, M 4} / g_{m, M 3}
$$

a pair of complementary bias voltages, $V_{\mathrm{bP}}$ and $V_{\mathrm{bN}}$, can be achieved. If $n=1$ is chosen in equation (3.18) and $V_{\mathrm{C}}$ in equation (3.15) is replaced with $V_{\mathrm{bN}}$ in equation (3.17), the MDLL operation frequency can be expressed as,

$$
f_{M D L L}=\frac{1}{2 C_{G} R_{\text {tg_c } c r l}}=\left(\frac{1}{2 C_{G} k\left(\frac{\left(1-e^{-1}\right) V_{D D}}{g_{m, M 1}\left(V_{c t r l}-V_{t h}\right) / g_{m, M 3}-V_{t h}}-0.3\right)}\right)
$$

Transistor sizes used in the VCDL and its bias generation circuit are summarized in Table 
Table 3.4 Transistors used in the VCDL and the bias generation circuit.

\begin{tabular}{|c|c|c|c|}
\hline Device & $\mathrm{W} / \mathrm{L}(\mu \mathrm{m} / \mu \mathrm{m})$ & Device & $\mathrm{W} / \mathrm{L}(\mu \mathrm{m} / \mu \mathrm{m})$ \\
\hline$M 1$ & $30 / 0.2$ & NMOS in $M U X$ & $3 / 0.2$ \\
\hline$M 2$ & $20 / 0.2$ & PMOS in $\ln v 1,2,3$ & $25 / 0.2$ \\
\hline$M 3$ & $60 / 0.2$ & NMOS in $\operatorname{nn} v 1,2,3$ & $10 / 0.2$ \\
\hline$M 4$ & $60 / 0.2$ & PMOS in $T G$ & $10 / 0.2$ \\
\hline PMOS in MUX & $7.5 / 0.2$ & NMOS in $T G$ & $4 / 0.2$ \\
\hline
\end{tabular}

\subsection{Simulation Results}

The proposed MDLL is designed in the TSMC CMOS $0.18 \mu \mathrm{m}$ process and simulated with Advanced Design System (ADS). Because 3600Mbps DDR4 controller are still in the research stage for most DDR vendors, the operation frequency of the proposed MDLL is set to $1.8 \mathrm{GHz}$ with a $150 \mathrm{MHz}$ reference signal and a division ratio of 12 . The functionality of period to voltage conversion in the PD can be observed from the MDLL output $f_{\mathrm{ob}}$ and the converted voltage pulse-train signal $V_{-}$int0 shown in Figure 3.23. Figure 3.23(a) shows that in the initial locked state, the $12^{\text {th }}$ cycle of $f_{\text {ob }}$ has a smaller period which results in a lower voltage pulse shown in the waveform of $V_{-}$int 0 . The lower pulse is sampled to generate $V_{\text {inst }}$.

Figure 3.23(b) shows that when the MDLL operates in the stable state, the $12^{\text {th }}$ cycle of $f_{\mathrm{ob}}$ 
has a similar period to the rest of the cycles and this also means a similar height voltage pulse generated in $V_{-}$into.

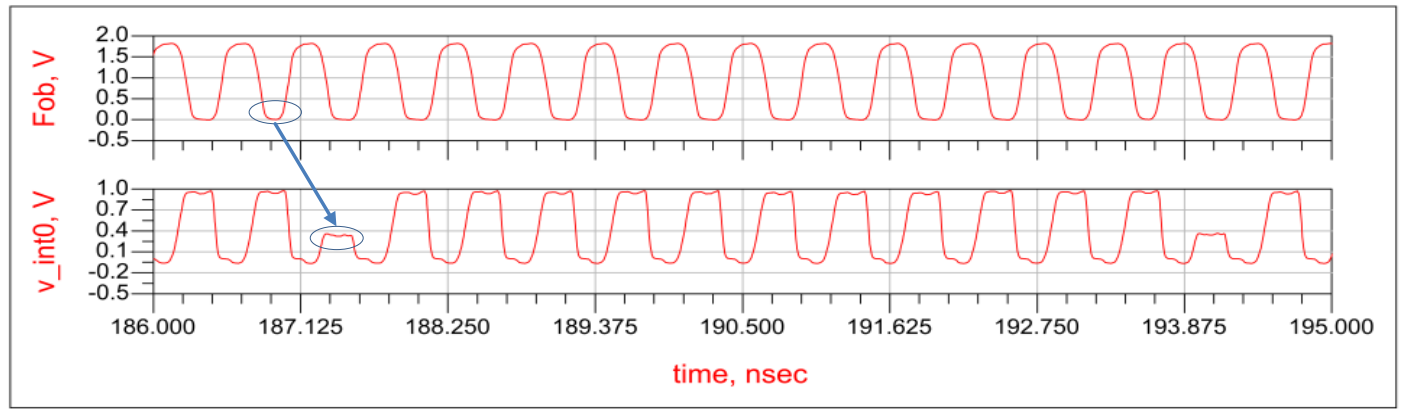

(a)

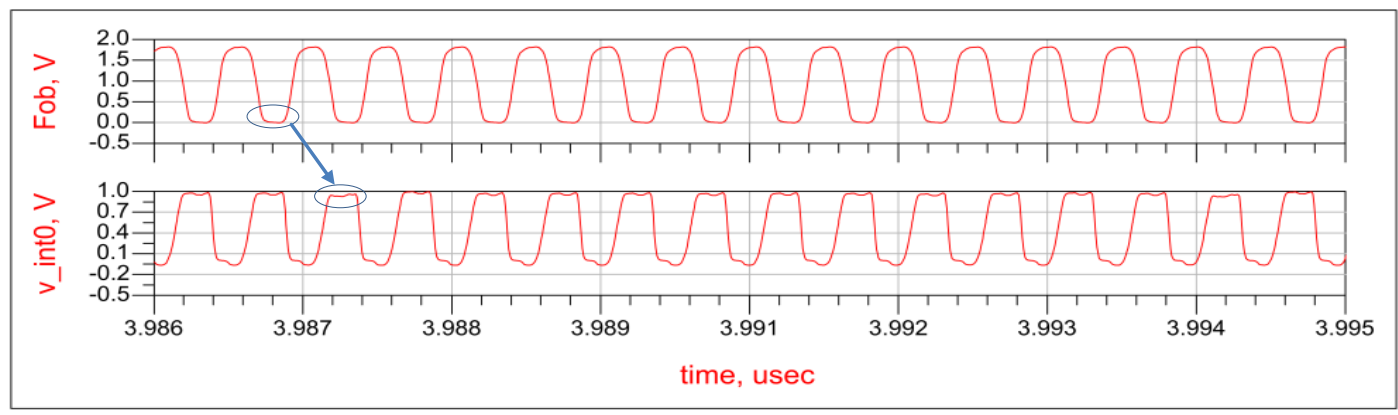

(b)

Figure 3.23: MDLL output $f_{\mathrm{ob}}$ and the converted voltage pulse-train signal $V_{\text {into }}$. (a) In the initial locked state, the $12^{\text {th }}$ cycle has a smaller period than the rest of the cycles; (b) In stable state, the $12^{\text {th }}$ cycle has a similar period to the rest of the cycles.

The spectrum of the MDLL output is shown in Figure 3.24. It is read that the reference spur is $-60.1 \mathrm{dBc}$. The SPO can be calculated by equation (3.6),

$$
\tau \approx T_{\text {out }} \times 10^{\text {spur }(B B c) / 20}=1 / 1.8 G H z \times 10^{(-60.1 / 20)}=0.55 p s\left(p k_{-} p k\right)
$$


A conventional MDLL has been designed by replacing the PD and V/I blocks with the PFD and charge pump in [13]. Simulated with the same division ratio of 12 , and the same reference frequency of $150 \mathrm{MHz}$, the spectrum of the conventional MDLL output is shown in Figure 3.25. The reference spur is $-30.1 \mathrm{dBc}$. Thus the deterministic jitter is $17.4 \mathrm{ps}(\mathrm{pk}-\mathrm{pk})$. Compared with the conventional MDLL, the proposed one achieves a $30 \mathrm{~dB}$ reference spur reduction. The reference spur reduction performance comparison is shown in Figure 3.5. Because the unit $\mathrm{dBc}$ is a relative value and does not reflect the absolute value. The reference spur has been converted to deterministic jitter according to the output frequency. It can be seen that the proposed method achieved the smallest deterministic jitter compared with the published works.

A type II PLL with the same division ratio of 12 and the same division circuit used in the MDLL has been designed. The loop filter is a $550 \Omega$ resistor in series with a $25 \mathrm{pF}$ capacitor and in parallel with a $1.25 \mathrm{pF}$ capacitor. The charge pump is the same as the one used in [13]. In order to check the high frequency power supply induced jitter performance, a $1.8 \mathrm{~V}$ DC power supply voltage with a $1 \mathrm{GHz} 60 \mathrm{mV}$ peak-to-peak amplitude sawtooth-shaped noise signal is added to both the PLL and the MDLL as the power supply. A sawtooth-shaped noise signal provides both even and odd harmonic components. The simulation results of the output jitter sequences of the PLL and the MDLL are plotted in Figure 3.26. It can be seen 
that the peak-to-peak jitters are 7.81ps and 11.3ps for the MDLL and the PLL respectively.

Compared to the PLL, the MDLL has a better PSIJ performance when both have the same $1 \mathrm{GHz} 60 \mathrm{mV}$ peak-to-peak amplitude sawtooth-shaped noise signal on the supply voltage.

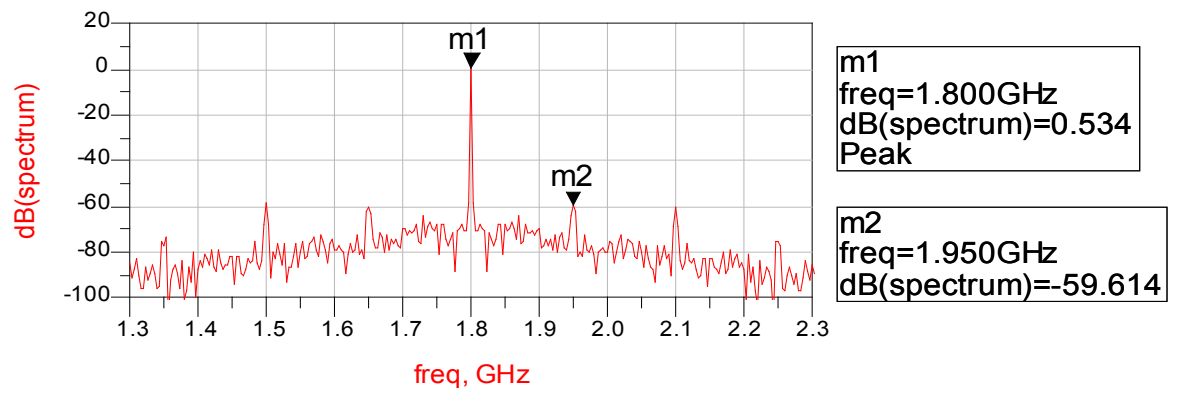

Figure 3.24: Spectrum of the proposed MDLL output.

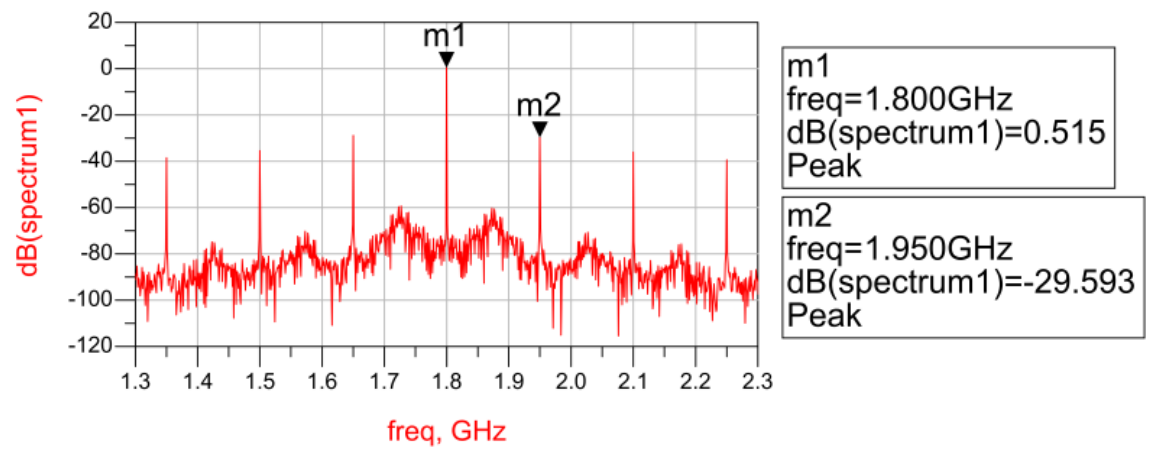

Figure 3.25: Spectrum of the conventional MDLL output. 


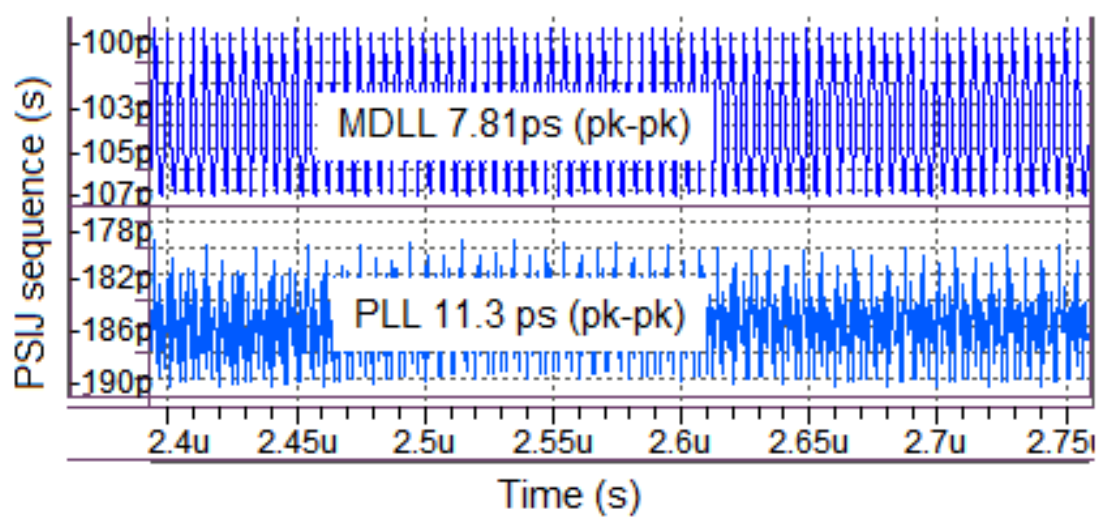

Figure 3.26: Jitter sequence plots for MDLL and PLL with $60 \mathrm{mV}$ peak-to-peak sawtooth shaped supply noise

Table 3.5 Deterministic jitter comparison with published works.

\begin{tabular}{|c|c|c|c|c|c|}
\hline & {$[13]$} & {$[15]$} & {$[16]$} & {$[51]$} & This work \\
\hline Technology & $0.18 \mu \mathrm{m}$ & $0.13 \mu \mathrm{m}$ & $65 \mathrm{~nm}$ & $0.13 \mu \mathrm{m}$ & $0.18 \mu \mathrm{m}$ \\
\hline $\begin{array}{c}\text { Output Frequency } \\
(\mathrm{GHz})\end{array}$ & 1.216 & 1.6 & 1.6 & 1.5 & 1.8 \\
\hline $\begin{array}{c}\text { Ref. frequency } \\
(\mathrm{MHz})\end{array}$ & 64 & 50 & 50 & 375 & 150 \\
\hline Division ratio & 19 & 32 & 32 & 4 & 12 \\
\hline Ref. spur (dBc) & -46.5 & -58.3 & -47 & -55.6 & -60.1 \\
\hline $\begin{array}{c}\text { Deterministic jitter } \\
(\mathrm{ps} \text { pp) }\end{array}$ & 3.89 & 0.76 & 2.79 & 1.11 & 0.55 \\
\hline
\end{tabular}




\subsection{Summary and discussion}

\subsubsection{Summary}

A static phase offset reduction technique for MDLL has been proposed. The technique has been verified by a behavioral Simulink model. The reduced equivalent SPO is measured by the power of reference spur level.

A design technique of reducing MDLL reference spurs has been proposed. With a high gain stage inserted between the PD/PFD and charge pump, the equivalent SPO has been decreased by a factor equal to the gain of the gain stage. The gain stage is achieved through a specific phase detector which employs a period to voltage converter. A high-gain integrator converts the instantaneous period of the MDLL output into a voltage pulse train signal. From the pulse-train signal, the phase error is extracted and used to reduce the SPO through a feedback loop. The proposed technique is simulated in the TSMC CMOS $0.18 \mu \mathrm{m}$ process. Compared with a conventional MDLL, the proposed one achieves a $30 \mathrm{~dB}$ reference spur reduction. The simulation results verify the effectiveness and feasibility of the proposed design technique.

\subsubsection{Discussion}

Theoretically from Figure 3.3 (c) to (d) only the equivalent SPO has been reduced and the 
loop characteristic has not been changed. Any noise from the phase detector has been amplified by the gain stage with a factor of $G$ and also has been attenuated by the same value $G$ from charge pump. However, in the proposed MDLL as shown in Figure 3.5(a), the gain stage is achieved by a high-gain period-to-voltage converter as shown in Figure 3.5(b). Any noise shown at the input of the high-gain period-to-voltage converter will be amplified and shown as the increased phase noise at the output of the MDLL. In order to achieve a low phase noise, the gain of the V/I stage shown in Figure 3.17 should be much smaller to attenuate the amplified noise, which means that a relatively narrower bandwidth is necessary for the proposed MDLL. A narrower bandwidth leads to a longer locking time. As a result, a longer simulation time is needed. Because this design is to verify the concept of inserting gain stage leading to reduced equivalent SPO, the bandwidth has been traded off with simulation time. Consequently a relatively large phase noise has been seen in Figure 3.24. Compared with the clock spectrum shown in Figure 3.24, Figure 3.25 shows a relatively clean clock spectrum which is generated from a conventional MDLL without an inserting gain stage. 


\section{Chapter 4}

\section{Power Supply Induced Jitter Sensitivity of a Buffer Chain}

\subsection{Introduction}

To achieve a high jitter performance DDR controller, a reduced reference spur MDLL is proposed and verified in the previous chapter. However, after a low jitter clock is generated, it has to be delivered to the registers distributed over a very large physical area which means that large clock buffer chains have to be used for clock distribution. Clock buffer chains essentially can be modeled as a voltage controlled delay line and the control voltage is the power supply [22], which means that any variation on the power supply will be directly translated into jitter. As a result, the power supply induced jitter of the large clock buffer chains is significant.

As transmission speed surpasses multi-gigabits per second for I/O interfaces, to meet the stringent timing budget, power supply induced jitter has attracted many research efforts [4-10] in the past few years. For power supply induced jitter (PSIJ) prediction, the conventional alpha method [21] is too pessimistic; the most accurate method is proposed in [23] where 
circuit simulation extracts the power supply induced jitter sensitivity (PSIJS) $S(f)$, then the jitter spectrum of the circuit can be obtained by $J(f)=S(f) V(f)$, where $V(f)$ is the power supply noise spectrum. In the following section, the alpha method and PSIJS analysis method will be discussed.

\subsection{PSIJ Estimation Method}

\subsubsection{Alpha Factor Method}

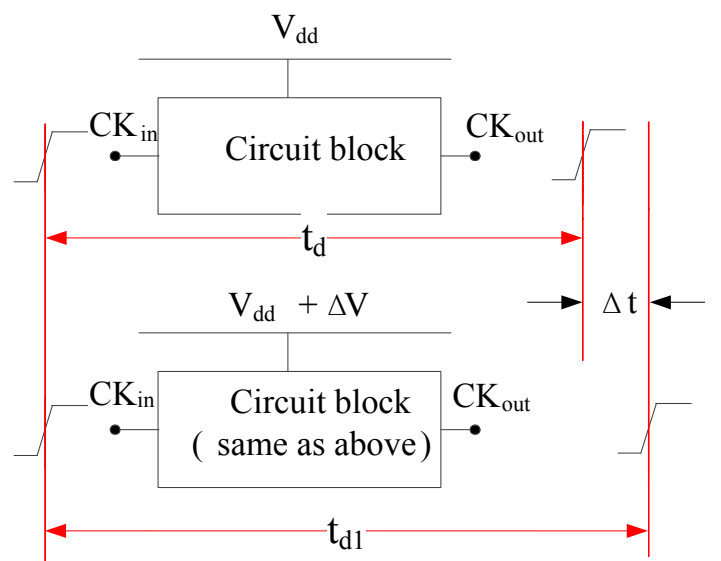

Figure 4.1: Method of measuring $\alpha$ factor.

The alpha factor method has been widely used in industry for calculating jitter in buffer chains caused by power supply variation [62]. The method of measuring alpha factor is shown in Figure 4.1. Firstly, the supply voltage of a buffer chain is set to be the nominal value, Vdd, and then the propagation delay, td, is obtained after running a simulation. Next a small DC voltage $\Delta \mathrm{V}$ (e.g $20 \mathrm{mV}$ ) is added to the nominal supply voltage and the second 
propagation delay $t_{\mathrm{d} 1}$ is obtained after running another simulation. The time difference between $t_{d 1}$ and $t_{d}$ is $\Delta t=t_{d 1}-t_{d}$. Then the $\alpha$ factor is defined as

$$
\alpha=\frac{\Delta t / t_{d}}{\Delta V / V_{d d}}
$$

During the circuit operation, if $\mathrm{V}_{\mathrm{dd}}$ and its ground have a differential peak-to-peak noise $\Delta \mathrm{V}$ then the PSIJ of the system can be estimated as

$$
P S I J=\alpha \cdot \Delta V \cdot\left(t_{d} / V_{d d}\right)[p s]
$$

Because the supply variation $\Delta \mathrm{V}$ is assumed to be a $\mathrm{DC}$ value, this method is too pessimistic for the higher frequency noise. It has become more difficult to accept the pessimism in jitter budgets for high speed data links.

\subsubsection{Power Supply Induced Jitter Sensitivity (PSIJS)}

To accurately calculate jitter budgets, Schmitt et al. [63] in 2007 proposed a new method for interface systems. The core of the method is the concept of power supply induced jitter sensitivity (PSIJS) whose definition is the ratio of the jitter generated by a single-frequency supply noise signal divided by the amplitude of this noise signal. The unit of jitter sensitivity is $[\mathrm{ps} / \mathrm{mV}]$ and it is a function of the noise frequency. The definition is expressed in (4.3) (this definition appears as (2) in [23]),

$$
\operatorname{PSIJS}(f)=\frac{\operatorname{Jitter}(f)}{\operatorname{Noise}(f)}[\mathrm{ps} / \mathrm{mV}]
$$


PSIJS can be obtained by measurements on silicon or by simulation of pre/post-layout of the transistor level circuit. To measure the noise sensitivity, a single-tone small signal sine wave is added to the DC power supply and then the resulting peak-to-peak jitter at this frequency is measured. By sweeping the noise frequency over the frequency range of interest the noise sensitivity profile can be plotted.

\subsubsection{PSIJ Analysis Methodology}

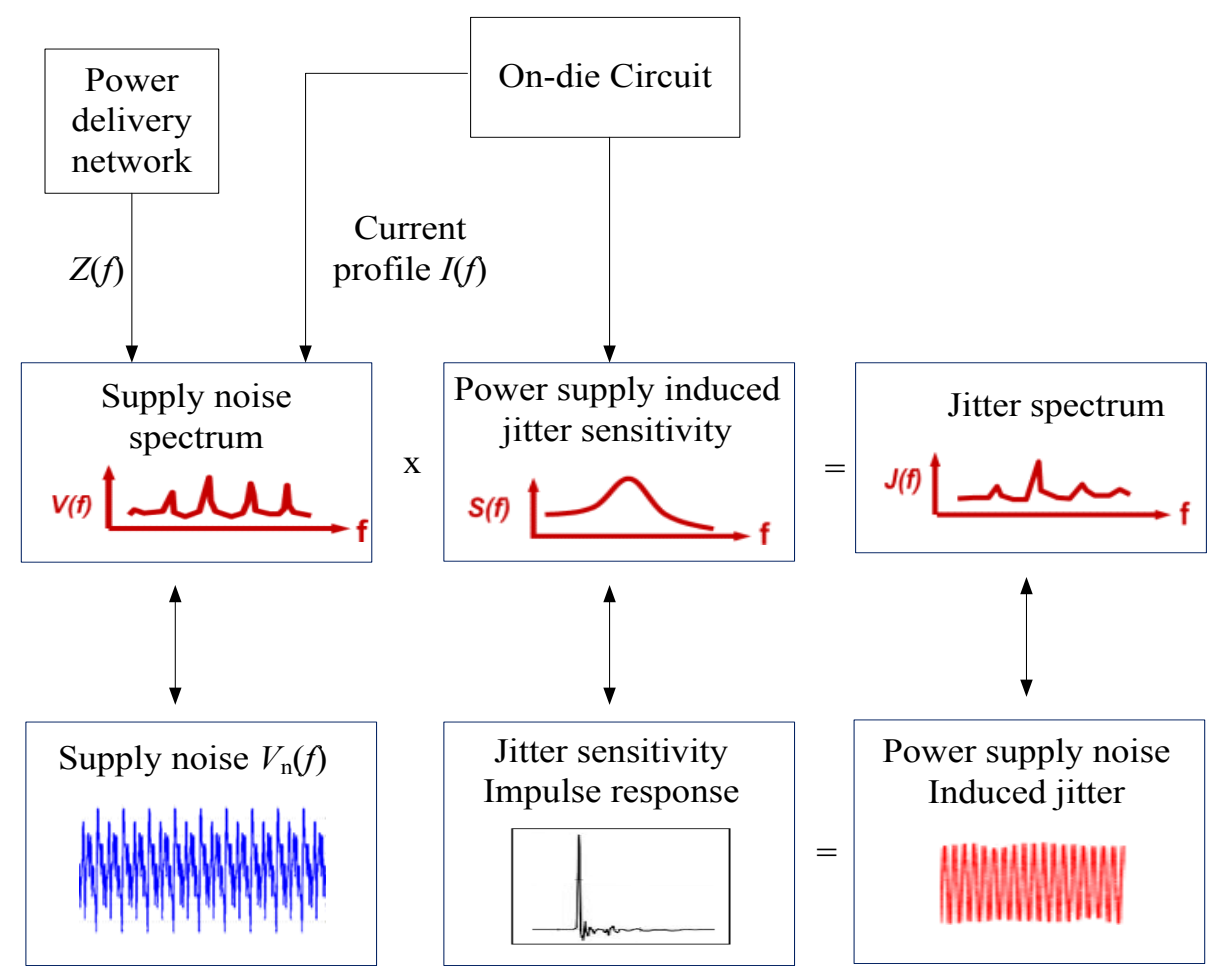

Figure 4.2: Methodology to predict power supply induced jitter.

The PSIJ prediction methodology is proposed in [23]. The methodology block diagram is 
shown in Figure 4.2. The two key components required are the circuit implementation and the power delivery network. The core is jitter sensitivity profile $S(f)$ which is directly characterized from the circuit implementation and is not related to the PDN. This brings the system design engineers a significant design technique. The conventional approach that only improves PDN is not cost effective [2]. Therefore, PSIJ must be controlled at the architecture level including both PDN and the circuit. A design technique proposed in [64] dramatically reduces PSIJ with minimal power increase and area over head by shaping the current signature, which can avoid the noise contents hitting PDN resonance which has high impedance.

In Figure 4.2, the supply noise profile $V_{\mathrm{n}}(f)$ is determined by the interaction between the current profile $I(f)$ and the power delivery network (PDN). It can be calculated either by multiplying the current spectrum $I(f)$ with the PDN impedance profile $Z(f)$ or by performing fast Fourier transform (FFT) on the time domain supply voltage noise waveform generated by transient simulation. In general, the supply noise spectrum is circuit activity and PDN implementation dependent. In addition to this, the system jitter sensitivity to supply noise is only determined by the circuit implementation. The jitter sensitivity is a system transfer function, mapping the supply noise as stimulus to the PSIJ impact as a response. It is independent of data activity or PDN realization. This allows jitter sensitivity extraction to 
analyze the clocking circuit alone. The next step is to multiply the supply noise spectrum and the jitter sensitivity profile to obtain the PSIJ spectrum $J(f)$ which provides significant insight into the system PSIJ in revealing the major jitter contributors in the frequency domain. The $J(f)$ can be further used to derive the time domain jitter sequence by performing the inverse fast Fourier transform (IFFT) on it.

\subsection{Overview of Power Supply Induced Jitter Sensitivity of a Buffer Chain}

Due to a large amount of buffer insertion in today's designs, especially in clock and timing circuits, the buffer delays account for a large percentage of all critical timing nets in a design. Jitter, variation of the buffer delay, is one of the most important challenges in these designs. In addition to this, scaling of power supply levels and improving transconductance of devices have increased the sensitivity of buffers to power supply induced delays [65].

Since the PSIJS analysis method provides a significant technique to estimate PSIJ, the PSIJS of clock buffers has been investigated in recent years. PSIJ analysis methodology is proposed in [23] but PSIJS of buffer chains is not studied. In [26,27], PSIJS of a single inverter is provided. The PSIJS is analytically derived in closed form expressions for a single-ended buffer. The analytic transfer functions are derived from a linear differential equation obtained from asymptotic linear inverter I-V curves. Figure 4.3 shows an inverter 
with a load capacitor, $\mathrm{C}$, and the voltage expressions for both noisy power supply and ground. The illustration of input and output waveforms for the low-to-high output transition is also shown in Figure 4.3.
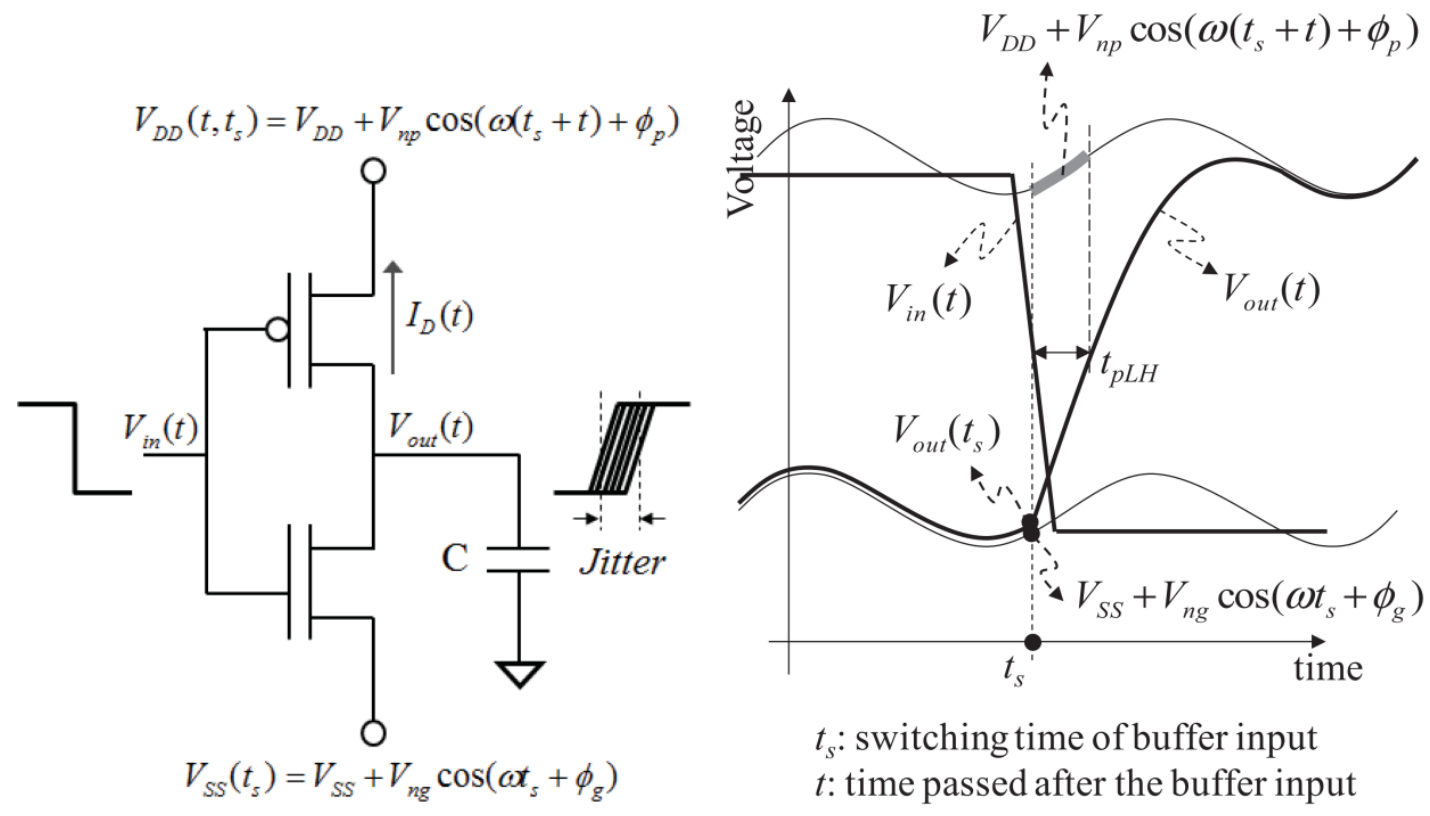

Figure 4.3: Effect of power and ground voltage fluctuations on the propagation delay for the low-to-high output transition and the representation of each voltage waveform [26] (C) 2013 IEEE.

When the magnitude of the $V_{\mathrm{DS}}$ is sufficiently large during the initial output transition, the drain current in the saturation region can be approximately represented in a linear function of the gate-source and drain-source voltages as (4.4) (this equation appears as (5) in [26]),

$$
\begin{aligned}
& \quad I_{\mathrm{D}}(t)=f\left(V_{\mathrm{GS}}(t), V_{\mathrm{DS}}(t)\right)=-G_{\mathrm{mp}} V_{\mathrm{DD}}-g_{\mathrm{mp}} V_{\mathrm{np}} \cos \left(\omega\left(t+t_{\mathrm{s}}\right)+\varphi_{\mathrm{p}}\right)+\lambda_{\mathrm{p}}\left(V_{\mathrm{out}}(t)-\right. \\
& \left.V_{\mathrm{DD}}-V_{\mathrm{np}} \cos \left(\omega\left(t+t_{\mathrm{S}}\right)+\varphi_{\mathrm{p}}\right)\right)
\end{aligned}
$$


where the $G_{\mathrm{mp}}$ and $g_{\mathrm{mp}}$ are the large signal and the small signal gains from the pMOS gate-source voltage, respectively, and $\lambda_{\mathrm{p}}$ is the transconductance of the pMOS relative to the drain-source voltage. When the drain current is written as a linear function of the gate-source and drain-source voltages, the effect of threshold voltage can be neglected.

Also, the drain current charging the loading capacitor, $\mathrm{C}$, is a function of the output voltage as (4.5) (this equation appears as (6) in [26]),

$$
I_{D}(t)=-C \frac{d V_{\text {out }}(t)}{d t}
$$

From the above equation (4.4) and (4.5), the PSIJS functions for low-to-high and high-to-low output transitions can be expressed as (4.6) (this equation appears as (28-31) in [26]), $\left\{\begin{array}{l}H_{L H}^{\text {ground }}(\omega)=\frac{C}{G_{m p}+\lambda_{p}} \frac{1}{\sqrt{C^{2} r_{o n n}^{2} \omega^{2}+1}} \frac{1}{V D D} \times \angle\left(\pi-a \tan 2\left(C r_{\text {onn }} \omega, 1\right)\right) \\ H_{L H}^{\text {power }}(\omega)=\frac{C}{0.5 \lambda_{p}+G_{m p}} \frac{\lambda_{p}+g_{m p}}{\lambda_{p}^{2}+C^{2} \omega^{2}} \frac{1}{V D D} \times \sqrt{A(\omega)^{2}+B(\omega)^{2}} \angle(\pi-a \tan 2(B(\omega), A(\omega))) \\ H_{H L}^{\text {ground }}(\omega)=\frac{C}{G_{m n}+\lambda_{n}} \frac{1}{\sqrt{C^{2} r_{o n p}^{2} \omega^{2}+1}} \frac{1}{V D D} \times \angle\left(-a \tan 2\left(C r_{\text {onp }} \omega, 1\right)\right) \\ H_{H L}^{\text {power }}(\omega)=\frac{C}{0.5 \lambda_{n}+G_{m n}} \frac{\lambda_{n}+g_{m n}}{\lambda_{n}^{2}+C^{2} \omega^{2}} \frac{1}{V D D} \times \sqrt{A(\omega)^{2}+B(\omega)^{2}} \angle(-a \tan 2(B(\omega), A(\omega)))\end{array}\right.$ 


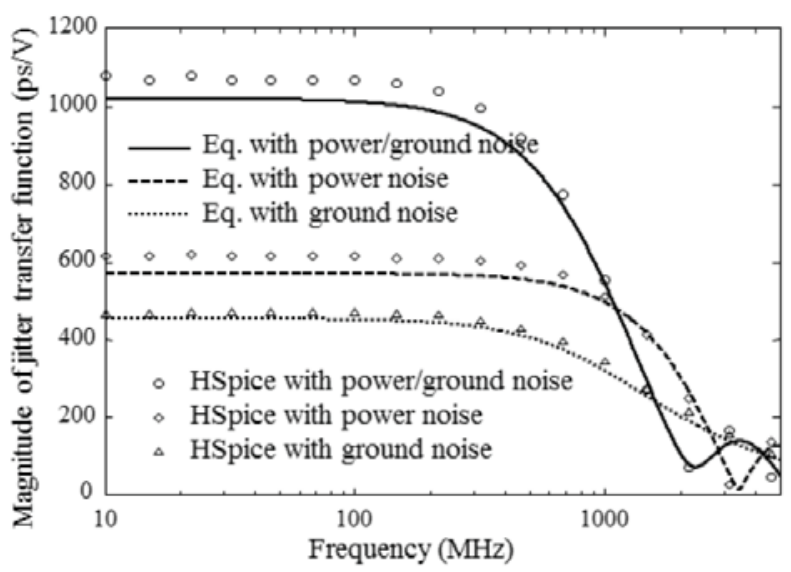

Figure 4.4: Transfer functions of supply voltage fluctuation to jitter obtained from HSPICE simulation and analytical expression [26] (C) 2013 IEEE.

The above PSIJS expression of (4.6) is effective for a single inverter. The plots of analytical expression (4.6) and Hspice sim results are shown in Figure 4.4.

In [67], a method similar to [26,27] has been used to analyze a two-stage buffer. The analytical expressions of the output waveforms have been obtained by solving the differential equations with each frequency component of the supply voltage fluctuations. The output waveforms in the time domain are then obtained by combining every contribution of all the frequency components, but this could be too complicated and impractical for engineering design. In [66], the output voltage waveforms of the multistage buffers are calculated but PSIJS of multistage buffers are not derived. PSIJ of a voltage controlled delay line shown in Figure 4.5 is plotted in [28] but an explicit expression of PSIJS is not provided. 


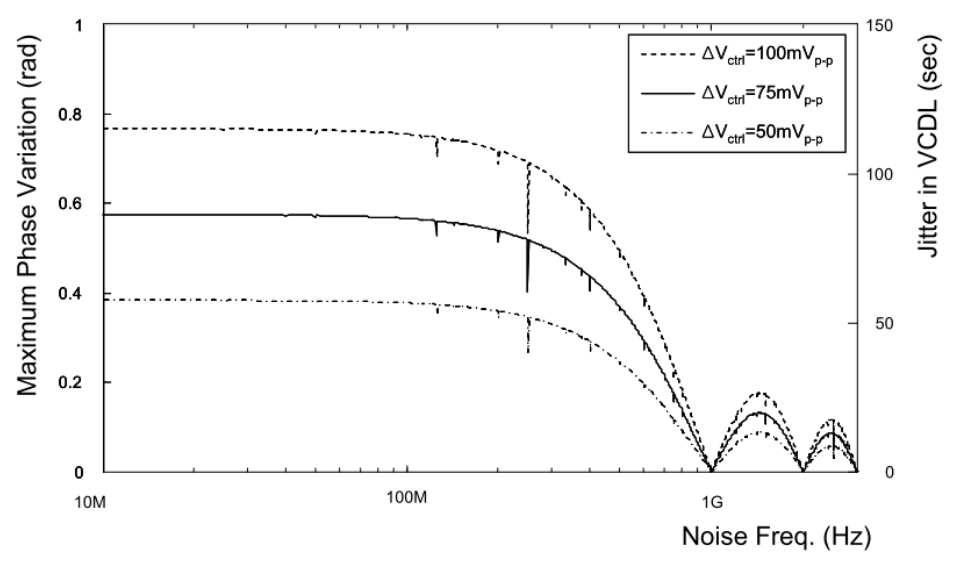

Figure 4.5: Phase variation and jitter in the VCDL depending on the variation of the control voltage. The dashed line implies the jitter with $100 \mathrm{mVp}$-p of the variation of the control voltage. The solid line and the wavy line show the jitter with $75 \mathrm{mVp}-\mathrm{p}$ and $50 \mathrm{mVp}-\mathrm{p}$ of the control voltage, respectively [28] (C) 2014 IEEE.

In the dissertation, an analytical expression of power supply induced jitter sensitivity of a buffer chain is derived, analyzed and compared with simulation results. The transfer function is mainly a function of the maximum and minimum propagation delay of the buffer chain. The function can be easily obtained and used in jitter budget calculation.

\subsection{Derivation of PSIJS of a buffer chain}

A buffer chain with a noisy power supply is shown in Figure 4.6. The noisy power supply is assumed to be a DC power supply with a single tone small signal AC noise. To the first-order approximation, the propagation delay change of a buffer chain due to small signal supply noise can be expressed as [62],

$$
\Delta T_{P}=K_{b u f} \cdot \Delta V_{D D}
$$


where $\Delta T_{P}$ is the delay change, $\Delta V_{D D}$ the supply variation and $K_{b u f}$ a static coefficient which is related to the buffer chain device size and technology. Analogous to the delay gain of a VCDL, here $K_{b u f}$ is the small signal delay gain of a buffer chain. Therefore, the total delay of the buffer chain can be expressed as,

$$
T_{P}=T_{P 0}+\Delta T_{P}=T_{P 0}+K_{b u f} \cdot \Delta V_{D D}
$$

where $T_{P 0}$ is the nominal propagation delay when power supply is at DC nominal voltage, $V_{D D 0}$, shown in Figure 4.6. So essentially a buffer chain is a VCDL and the control voltage is the supply voltage. Under the small signal noise condition, $T_{P 0} \gg \Delta T_{P}$.

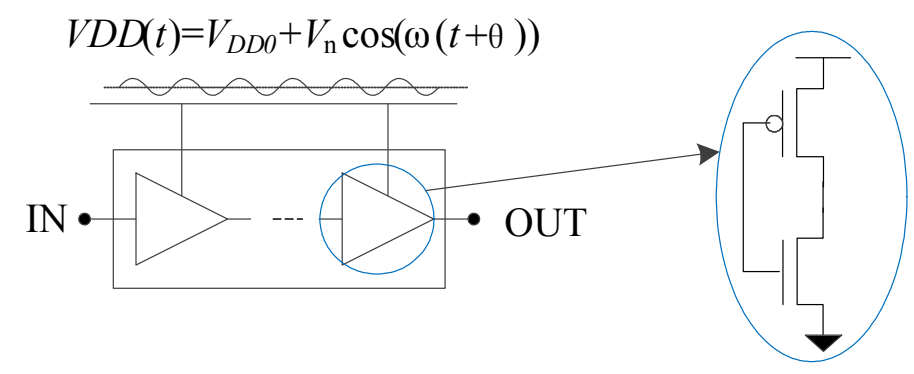

Figure 4.6: A buffer chain with a noisy power supply.

\subsubsection{Equivalent RC Model of a Buffer Chain}

The nominal propagation delay of a buffer chain can be obtained through an equivalent RC circuit model. The equivalent $\mathrm{RC}$ circuit models for $\mathrm{nMOS}$, pMOS transistors and an inverter [68] are shown in Figure 4.7. For the inverter, either nMOS or pMOS is on. In 
Figure $4.7, k, k_{1}$ and $k_{2}$ are the gate widths respectively. A unit nMOS transistor is defined to have effective resistance $R$. A unit pMOS transistor has greater resistance, generally in the range between $2 R$ and $3 R$ due to its lower mobility. For simplicity, here we assume that the resistance of a unit pMOS transistor is $2 R$. For Fig. 2(b), if $k_{1}=k_{2}$, the propagation delay from $X$ to $Y$ is,

$$
T_{P(X, Y)}=\left(R / k_{1}\right) \cdot 3\left(k_{1}+k_{2}\right) C \cdot \ln 2=6 R C \ln 2
$$

If $k_{1} \neq k_{2}$, the propagation delay from $X$ to $Y$ is,

$$
T_{P(X, Y)}=\left(R / k_{1}\right) \cdot 3\left(k_{1}+k_{2}\right) C \cdot \ln 2=3 R C\left(1+k_{2} / k_{1}\right) \ln 2
$$

From (4.10) one can see that if the buffer sizes of each stage are not exactly the same, the large signal propagation delay is not homogeneous.

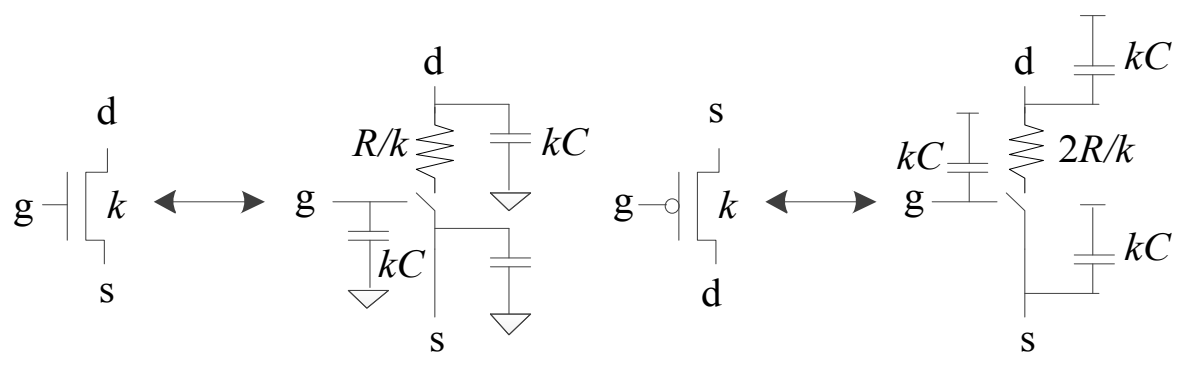

(a)

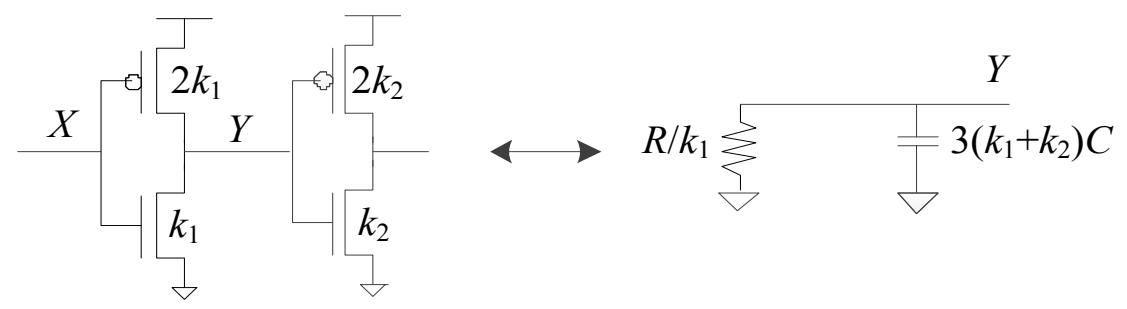

(b)

Figure 4.7: Equivalent RC circuit model. (a) nMOS and pMOS transistor models; (b) An inverter model [68]. 


\subsubsection{Small Signal Equant Delay Gain Model of a Buffer Chain}

The total delay of a buffer chain is the sum of the delays of each stage [68]. So (4.8) can be expressed with the delays of each stage,

$$
T_{P}=\sum_{i=1}^{M} T_{P 0, i}+K_{b u f, i} \cdot \Delta V_{D D, i}
$$

where $T_{P 0, I}$ is the $i$-th stage nominal propagation delay, $K_{b u f, I}$ the $i$-th stage delay gain, $\Delta V_{D D, i}$ the supply variation of $i$-th stage and $M$ the total stage number.

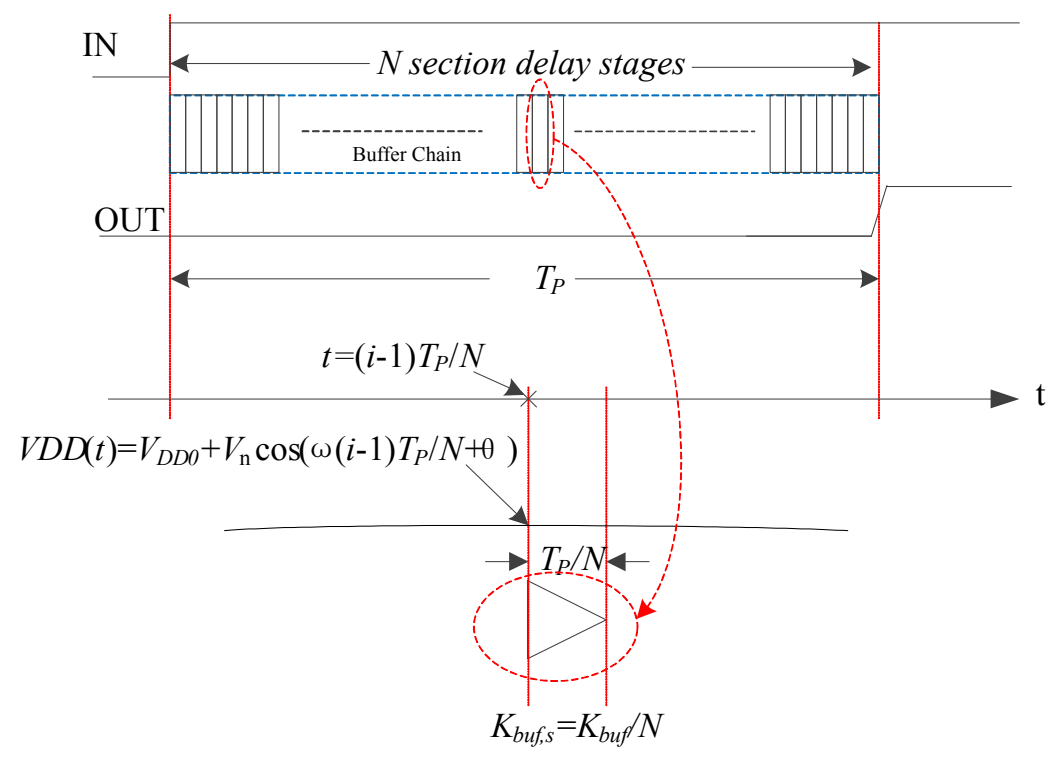

Figure 4.8: Buffer chain equivalent $N$ sections of small delay stages and the $i$-th section of delay stage.

Since the delay gain of each stage is a static coefficient, for the $i$-th stage, delay gain $K_{\text {buf,I }}$ can be equally divided into $N_{i}$ segments, where $N_{i}$ is an arbitrarily large integer number. 
If the following condition is satisfied,

$$
\frac{K_{b u f, 1}}{N_{1}}=\frac{K_{b u f, 2}}{N_{2}}=\mathrm{L}=\frac{K_{b u f, M}}{N_{M}}
$$

a buffer chain with $M$ stages can be modeled as composing $N$ small sections and the delay gain of each section, $K_{b u f, s}$, is equal and can be expressed as,

$$
K_{b u f, s}=\frac{K_{b u f, i}}{N_{i}}
$$

where $N=\sum_{i=1}^{M} N_{i}$. Figure 4.8 shows the equivalent $\mathrm{N}$-section delay stages.

\subsubsection{Derivation of PSIJS of a Buffer Chain}

Because the noise on the supply voltage $V D D(t)$ is small signal and the delay gain of each small section (shown in Figure 4.6) is equal, the propagation delay of each small section can be approximated as,

$$
T_{P, i} \approx\left(T_{P 0}+\Delta T_{P}\right) / N \approx T_{P 0} / N
$$

thus $T_{P}$ can be expressed as,

$$
\begin{gathered}
T_{P} \approx \sum_{i=1}^{N} T_{P 0, i}+\sum_{i=1}^{N} \frac{K_{b u f}}{N}\left(V_{n} \cos \left(\omega(i-1) \frac{T_{P 0}}{N}+\theta\right)\right)=T_{P 0}+ \\
\frac{K_{b u f}}{N} \frac{\sin \theta-\sin \left(N \frac{T_{P 0}}{N} \omega+\theta\right)}{2}+\frac{K_{b u f}}{N} \frac{\cos \theta-\cos \left(N \frac{T_{P 0}}{N} \omega+\theta\right)}{2-2 \cos \left(\omega \frac{T_{P 0}}{N}\right)} \sin \left(\omega \frac{T_{P 0}}{N}\right)
\end{gathered}
$$

where $V_{\mathrm{n}}$ is the supply noise amplitude, $\omega$ the noise angular frequency, and $\theta$ the phase of 
noise at the rising edge of input signal. $\Theta$ can be of any value between 0 and $2 \pi$. The expression of power supply with a small signal AC noise can be found in Figure 4.6.

Since $N$ is arbitrarily large, one can take the number of $\mathrm{N}$ large enough to satisfy the following conditions: $\quad \frac{K_{b u f}}{N} \frac{\sin \theta-\sin \left(N \frac{T_{P 0}}{N} \omega+\theta\right)}{2} \rightarrow 0 \quad, \quad \sin \left(\omega \frac{T_{P 0}}{N}\right) \approx \omega \frac{T_{P 0}}{N} \quad$ and $\cos \left(\omega \frac{T_{P 0}}{N}\right) \approx 1-\left(\omega \frac{T_{P 0}}{N}\right)^{2} / 2$ !, thus (4.15) can be simplified to,

$$
T_{P}=T_{P 0}+K_{b u f} V_{n} \operatorname{sinc}\left(\pi f T_{P 0}\right) \sin \left(\pi f T_{P 0}+\theta\right)
$$

Since only the maximum jitter is of interest, we take $\sin \left(\pi f T_{P 0}+\theta\right)= \pm 1$. From (4.16), the maximum and minimum propagation delays can be approximated as,

$$
\begin{aligned}
& T_{P, \text { max }}=T_{P 0}+V_{n}\left|K_{b u f} \operatorname{sinc}\left(\pi f T_{P 0}\right)\right| \\
& T_{P, \text { min }}=T_{P 0}-V_{n}\left|K_{b u f} \operatorname{sinc}\left(\pi f T_{P 0}\right)\right|
\end{aligned}
$$

If the power supply noise is static with a value of $V_{\mathrm{n}}$, in other words, $V D D=V_{D D 0} \pm V_{\mathrm{n}}$, from (4.17) and (4.18) one can get

$$
\begin{aligned}
& T_{P, \text { max }, D C}=\left.T_{P, \text { max }}(f=0)\right|_{V D D=V_{D D 0}-V_{n}=V D D_{\min }}=T_{P 0}+V_{n}\left|K_{b u f}\right| \\
& T_{P, \text { min }, D C}=\left.T_{P, \text { min }}(f=0)\right|_{V D D=V_{D D 0}+V_{n}=V D D_{\max }}=T_{P 0}-V_{n}\left|K_{b u f}\right|
\end{aligned}
$$

where $T_{P, \max , D C}$ and $T_{P, \min , D C}$ are the propagation delays with $\mathrm{DC}$ supply voltage of $V D D_{\min }=V_{D D 0}-V_{\mathrm{n}}$ and $V D D_{\max }=V_{D D 0}+V_{\mathrm{n}}$

Jitter sensitivity is defined as the ratio between the jitter generated by a single-frequency supply noise signal divided by the amplitude of this noise signal [23]. So 
the magnitude of PSIJS is,

$$
\mid \text { Jitter_sensitivity }(f) \mid=\frac{T_{P, \max }(f)-T_{P, \min }(f)}{V_{n}-\left(-V_{n}\right)}
$$

From (4.17), (4.18), (4.19), (4.20) and (4.21) one can get,

Jitter_sensitivity $(f)=\frac{T_{P, \max , D C}-T_{P, \min , D C}}{V D D_{\max }-V D D_{\min }}\left|\operatorname{sinc}\left(\pi f \frac{T_{P, \max , D C}+T_{P, \min , D C}}{2}\right)\right|$

When $f=\mathrm{N}^{*} f_{\mathrm{IN}}(\mathrm{N}=0,1,2, \ldots)$, Jitter_sensitivity $(f)=0$, where $f_{\mathrm{IN}}$ is the frequency of buffer chain input signal, $I N$. This means that during each period of $I N$, the buffer chain sees exactly the same power supply waveform because at any input rising edge the supply noise has the same phase.

\subsubsection{Validation of the Derivation}

To validate (4.22), four buffer chains have been designed with TSMC CMOS processes. The transistor sizes, supply voltages and input signals for all the buffer chains are summarized in Table 4.1. $C_{\text {load }}$ is used to describe the capacitance which is connected to the output node of each single buffer stage, e.g. wire parasitic capacitance.

By plugging the supply voltage and propagation delay parameters (summarized in Table 4.1) into (4.22) and plotting the PSIJS, the results are shown in Figure 4.9 marked as "Theoretical result".

Based on the jitter sensitivity definition, Hspice simulation results are obtained through the following method: a single-tone small signal sine wave with an amplitude of $25 \mathrm{mV}$ (to 
satisfy the small signal noise assumption) is added to the DC power supply, $0.855 \mathrm{~V}$ (typical power supply voltage for TSMC28HPM18 process) for Buffer chain 1 and 2, and $1.8 \mathrm{~V}$ (typical power supply voltage for TSMC CMOS $0.18 \mu \mathrm{m}$ process) for Buffer chain 3 and 4 , then the resulting peak-to-peak jitter at this frequency is measured. Through sweeping the noise frequency over the frequency range of interest, the PSIJS's for all the buffer chains, marked as "Hspice sim result", are plotted and shown in Figure 4.9. 
Table 4.1 Transistor sizes, supply voltages and input signals summary of all buffer chains.

\begin{tabular}{|c|c|c|c|c|}
\hline \multirow{2}{*}{$\begin{array}{l}\text { Buffer } \\
\text { chain }\end{array}$} & 1 & 2 & 3 & 4 \\
\hline & \multicolumn{2}{|c|}{$\left(L_{\mathrm{N}}=L_{\mathrm{P}}=30 \mathrm{~nm}\right)$} & \multicolumn{2}{|c|}{$\left(L_{\mathrm{N}}=L_{\mathrm{P}}=0.2 \mu \mathrm{m}\right)$} \\
\hline Process & \multicolumn{2}{|c|}{ TSMC28HPM18 } & \multicolumn{2}{|c|}{ TSMC CMOS $0.18 \mu \mathrm{m}$} \\
\hline \multirow[b]{2}{*}{$\begin{array}{l}\text { Size-1 } \\
\text { Inverter }\end{array}$} & $\#^{*}=6$ & $\#=4$ & $\#=12$ & $\#=12$ \\
\hline & \multicolumn{2}{|c|}{$\begin{array}{l}W_{N}=0.5 \mu \mathrm{m} \\
W_{P}=1.0 \mu \mathrm{m} \\
C_{\text {load }}=1.2 \mathrm{fF}\end{array}$} & \multicolumn{2}{|c|}{$\begin{array}{c}W_{N}=0.3 \mu \mathrm{m} \\
W_{P}=0.8 \mu \mathrm{m} \\
C_{\text {load }}=5 \mathrm{fF}\end{array}$} \\
\hline \multirow[b]{2}{*}{$\begin{array}{l}\text { Size-2 } \\
\text { Inverter }\end{array}$} & $\#=0$ & $\#=4$ & $\#=4$ & $\#=4$ \\
\hline & \multicolumn{2}{|c|}{$\begin{array}{l}W_{N}=1.0 \mu \mathrm{m} \\
W_{P}=2.0 \mu \mathrm{m} \\
C_{\text {load }}=2.4 \mathrm{fF}\end{array}$} & \multicolumn{2}{|c|}{$\begin{array}{c}W_{N}=0.6 \mu \mathrm{m} \\
W_{P}=1.6 \mu \mathrm{m} \\
C_{\text {load }}=10 \mathrm{fF}\end{array}$} \\
\hline \multirow[b]{2}{*}{$\begin{array}{l}\text { Size-3 } \\
\text { Inverter }\end{array}$} & $\#=0$ & $\#=2$ & $\#=2$ & $\#=2$ \\
\hline & & $\begin{array}{l}2.0 \mu \mathrm{m} \\
4.0 \mu \mathrm{m} \\
=4.8 \mathrm{fF}\end{array}$ & & $\begin{array}{l}2 \mu \mathrm{m} \\
2 \mu \mathrm{m} \\
20 \mathrm{fF}\end{array}$ \\
\hline $\begin{array}{l}\text { Supply } \\
\text { voltage } \\
\text { (V) }\end{array}$ & \multicolumn{2}{|c|}{$\begin{array}{l}V D D_{\min }=0.83 \\
V D D_{\max }=0.88 \\
V_{\mathrm{DD} 0}=0.855\end{array}$} & \multicolumn{2}{|c|}{$\begin{array}{l}V D D_{\min }=1.775 \\
V D D_{\max }=1.825 \\
V_{\mathrm{DD} 0}=1.8\end{array}$} \\
\hline Load & $\begin{array}{l}1 \text { Size-1 } \\
\text { inverter }\end{array}$ & $\begin{array}{l}1 \text { Size-3 } \\
\text { inverter }\end{array}$ & $\begin{array}{l}1 \text { Size-3 } \\
\text { inverter }\end{array}$ & $\begin{array}{l}4 \text { Size-3 } \\
\text { inverters }\end{array}$ \\
\hline $\begin{array}{l}\text { Input } \\
\text { signal }\end{array}$ & \multicolumn{4}{|c|}{$\begin{array}{c}f=1.2 \mathrm{GHz} \quad \operatorname{Tr}(\text { rising edge })=0.1 \mathrm{ps} \\
\operatorname{Tf}(\text { falling edge })=0.1 \mathrm{ps}\end{array}$} \\
\hline Simulator & \multicolumn{4}{|c|}{ Hspice } \\
\hline $\begin{array}{l}T_{\mathrm{P}, \min , \mathrm{DC}} \\
T_{\mathrm{P}, \mathrm{max}, \mathrm{DC}}\end{array}$ & $\begin{array}{l}52.9 \mathrm{ps} \\
61.3 \mathrm{ps}\end{array}$ & $\begin{array}{l}95.5 \mathrm{ps} \\
112.7 \mathrm{ps}\end{array}$ & $\begin{array}{l}1328.6 \mathrm{ps} \\
1364.7 \mathrm{ps}\end{array}$ & $\begin{array}{l}1391 \mathrm{ps} \\
1431 \mathrm{ps}\end{array}$ \\
\hline
\end{tabular}

*\# --- Inverter numbers 


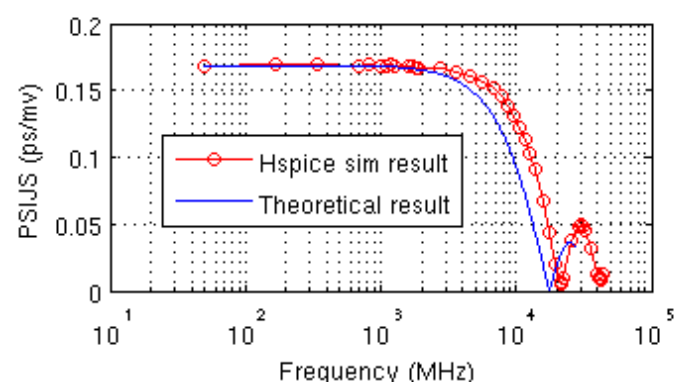

(a)

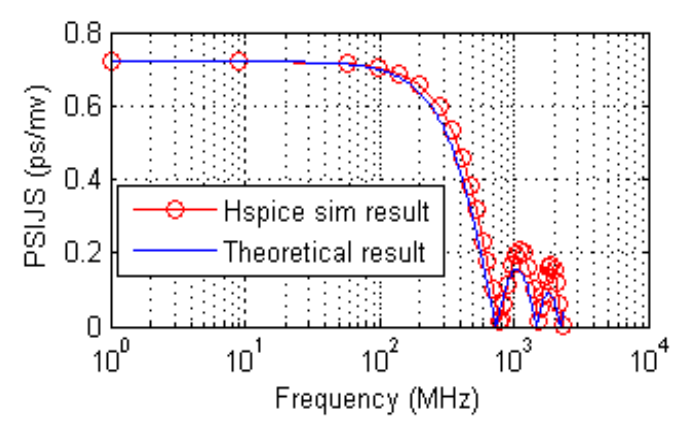

(c)

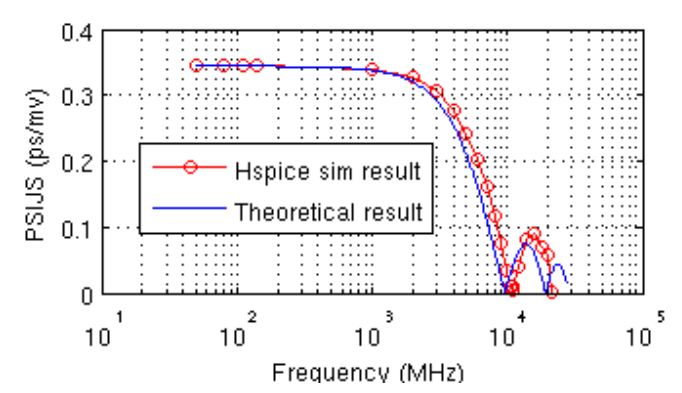

(b)

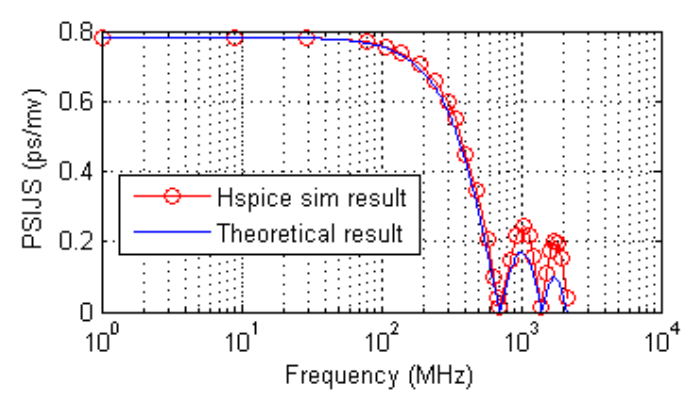

(d)

Figure 4.9: Plots of PSIJS from Hspice simulation results and expression (4.18). (a) Buffer chain 1; (b) Buffer chain 2; (c) Buffer chain 3; (d) Buffer chain 4.

From Figure 4.9 one can see that the calculated results from (4.22) match Hspice simulation results in a very wide range. For Buffer chain1, the jitter sensitivity is $0.169 \mathrm{ps} / \mathrm{mV}$ at low frequency and the first zero-crossing point of PSIJS is about $20 \mathrm{GHz}$. For Buffer chain2, the jitter sensitivity is $0.345 \mathrm{ps} / \mathrm{mV}$ at low frequency and the first zero-crossing point of PSIJS is about $10 \mathrm{GHz}$. For Buffer chain 3, the low frequency jitter sensitivity is $0.72 \mathrm{ps} / \mathrm{mV}$ and the first zero-crossing point is about $800 \mathrm{MHz}$. Buffer chain 4 is exactly the same as Buffer chain 3 but with a load 4 times larger. The low frequency jitter sensitivity for Buffer chain 4 is $0.782 \mathrm{ps} / \mathrm{mv}$ and the first zero-crossing point is about 
$700 \mathrm{MHz}$.

From the PSIJS plots of Buffer chain 1 and 2 one can see that the product of the PSIJS at low frequency and the zero-crossing frequency approximates a constant. This can also be concluded from (4.22). The zero crossing frequency of (4.22) is

$$
f_{0, x}=2 N /\left(T_{P, \max , D C}+T_{P, \min , D C}\right) \text {, where } N \text { can be any positive integer. For simplicity, only the }
$$

first zero-crossing point is considered. For any buffer chain, the zero crossing frequency will be,

$$
f_{0, x}=2 /\left(\alpha\left(T_{P, \max , D C}+T_{P, \min , D C}\right)\right)=2 /\left(\alpha T_{P, \max , D C}+\alpha T_{P, \min , D C}\right)
$$

where $\alpha$ is an arbitrary positive real number. So the maximum and minimum propagation delays become $\alpha T_{P, \max , D C}$ and $\alpha T_{P, \min , D C}$ respectively. The low frequency PSIJS will become

$$
P S I J=\frac{\alpha T_{P, \max , D C}-\alpha T_{P, \min , D C}}{V D D_{\max }-V D D_{\min }}
$$

The product of (4.23) and (4.24) becomes constant,

$$
\frac{2\left(T_{P, \max , D C}-T_{P, \min , \mathrm{DC}}\right)}{\left(T_{P, \max , D C}+T_{P, \min , D C}\right)\left(V D D_{\max }-V D D_{\min }\right)}=\text { constant }
$$

Because (4.25) is a constant, one can draw a commonsense conclusion that for a buffer chain the larger the propagation delay is, the larger jitter will be induced by the same power supply noise. The condition which must be satisfied for (4.25) is that the delay gains of each stage of the buffer chains to be compared with are approximately equal. This can be easily achieved with the same gate length for all the transistors in all buffer chains with the same 
CMOS process.

The phase of PSIJS at low frequency is $\pi$ or $-\pi$. This is because the maximum propagation delay always happens at $V D D_{\min }$ and the minimum propagation delay always happens at $V D D_{\max }$ if the noise frequency is relatively low compared with the first zero-crossing frequency of PSIJS which is inversely proportional to the averaged propagation delay.

\subsection{Comparison of PSIJ Estimation Methods}

In this section, the PSIJ calculation results from the conventional alpha factor method and PSIJS method are compared. The Buffer chain 4 in TABLE 4.1 is chosen. Power supply noise is $60 \mathrm{mVpp}$ sawtooth shape shown in Figure 4.10 and Figure 4.12 panel of "VDD". The noise signal period is $1 \mathrm{GHz}$.

According to (4.1) alpha factor is obtained,

$$
\alpha=\frac{\Delta t / t_{d}}{\Delta V / V_{d d}}=\frac{39.1 p s / 1411 p s}{50 m V p p / 1.8 V}=0.9976
$$

The PSIJ is calculated through (4.2) and (4.26),

$P S I J=\alpha \cdot \Delta V \cdot\left(t_{d} / V_{d d}\right)[p s]=0.9976 \times 60 m V_{p p}(1411 p s / 1.8 V)=46.9 p s$

According to the method in section 4.2, taking advantage of PSIJS plotted in Figure 4.9(d), the PSIJ is calculated through Matlab and the PSIJS sequences are plotted in Figure 
4.11. In Figure 4.11(a), the maximum peak to peak PSIJ is $8.3 \mathrm{ps}$ and in Figure 4.11(b), the maximum peak to peak PSIJ is $8.1 \mathrm{ps}$.

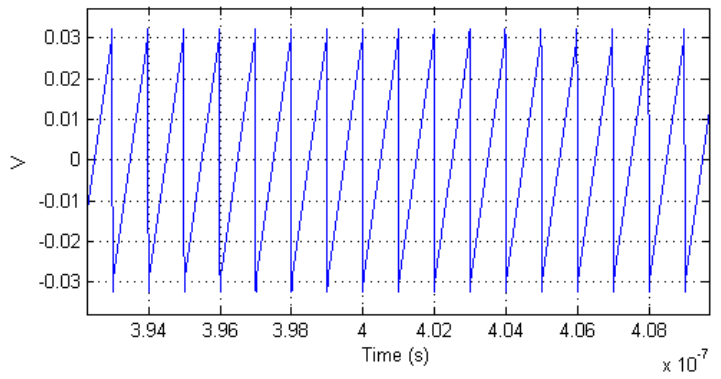

Figure 4.10: Buffer chain power supply sawtooth noise signal.

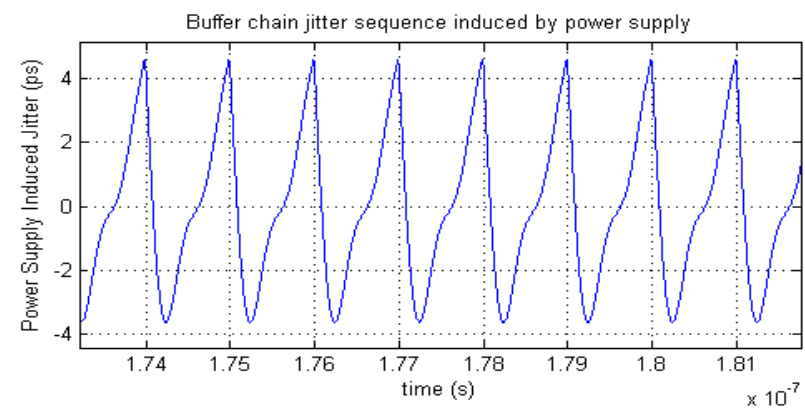

(a)

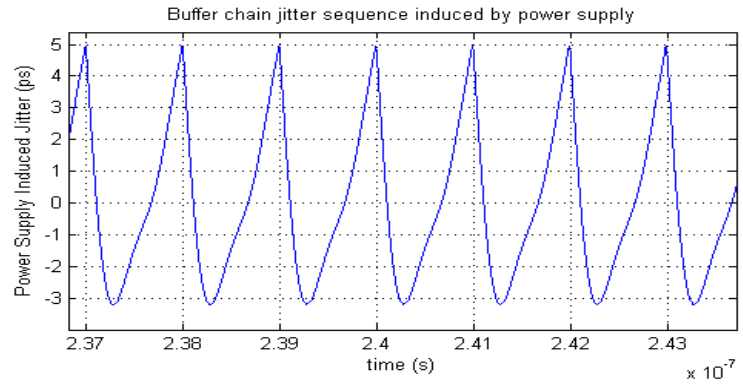

(b)

Figure 4.11: PSIJ sequences calculated through PSIJS of (a) Hspice result; (b) theoretical result.

In order to compare the calculated PSIJ results from alpha factor and PSIJS methods, circuit level simulation is carried on through Hspice simulator. The power supply noise is the 
same as that used in Matlab calculation, which is shown in Figure 4.12 panel "VDD". The PSIJ of the output waveform is plotted and shown in Figure 4.12 panel "JVST" and the peak to peak PSIJ is $8.7 \mathrm{ps}$. The PSIJ obtained from different methods are summarized in Table 4.2.

Table 4.2 Summary of the PSIJ obtained from different methods.

\begin{tabular}{|c|c|c|c|}
\hline & $\alpha$ factor method & PSIJS method & Hspice simulation \\
\hline PSIJ (ps, pk-pk) & 46.9 & 8.1 & 8.7 \\
\hline $\begin{array}{c}\text { Power supply } \\
\text { noise }\end{array}$ & \multicolumn{3}{|c|}{$60 \mathrm{mVpp}$ sawtooth-shaped with $1 \mathrm{GHz}$} \\
\hline
\end{tabular}

Comparing the PSIJ results obtained from different methods shown in Table 4.2, one can see that alpha factor method is too pessimistic and not accurate for PSIJ estimation when high frequency components are dominant in the power supply noise. The PSIJ obtained from PSIJS method is very close to that from circuit simulation. The PSIJS expression of (4.22) proposed in this dissertation leads to very high accuracy and quick PSIJ estimation. 


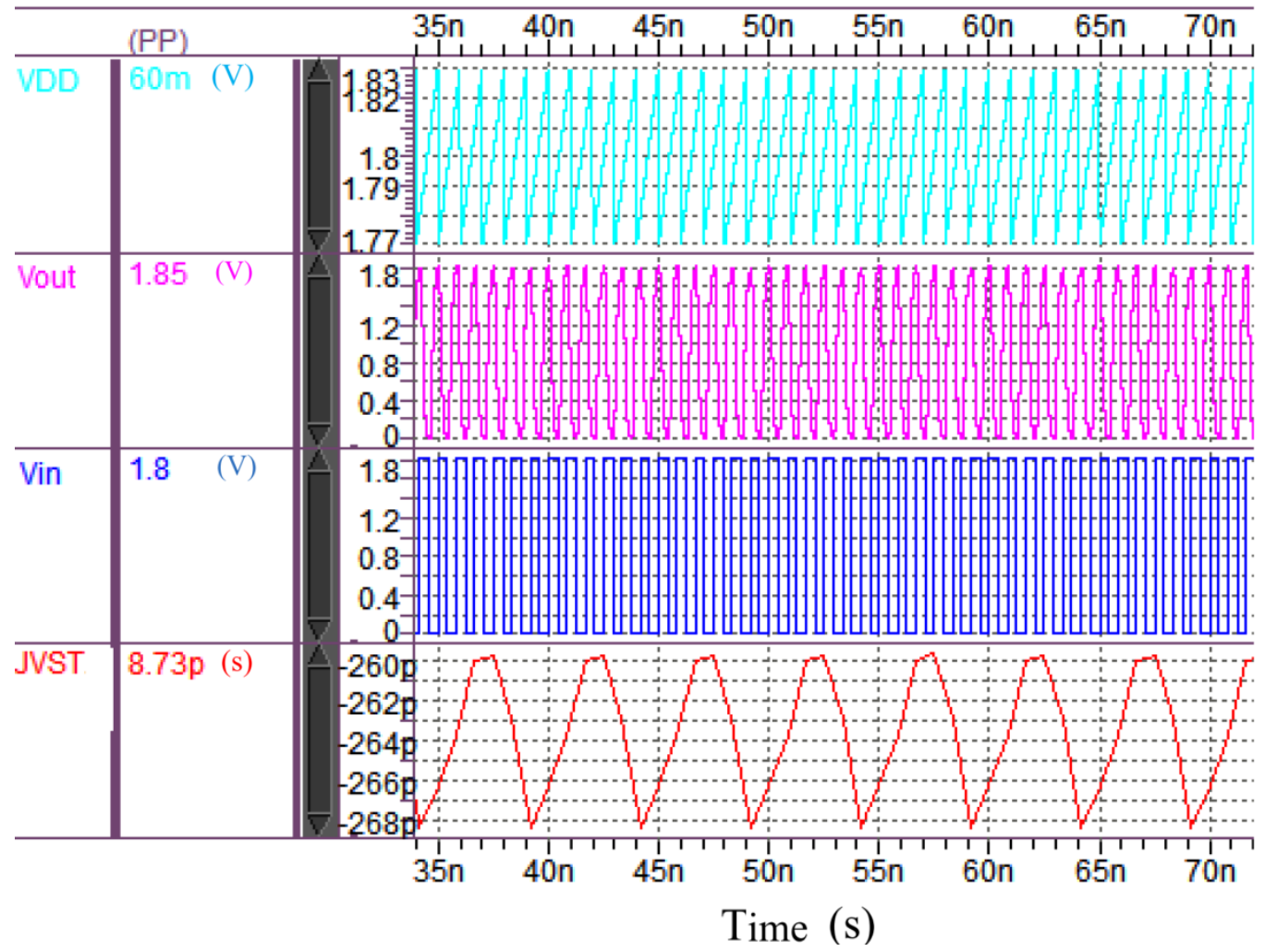

Figure 4.12: Hspice simulation results of PSIJ of buffer chain 4.

\subsection{Summary}

In this chapter, PSIJ estimation methods are briefly covered. The research on PSIJS of single buffer and multistage buffers has been reviewed. A simple and accurate expression of PSIJS for a CMOS buffer chain is presented. It is not necessary to know the buffer chain architecture and electrical parameters. Since the expression does not require the buffer chain architecture and electrical parameters, it can be used to quickly and accurately predict the amount of jitter induced by the supply noise of the long buffer chains in DDR controller 
application. 


\section{Chapter 5}

\section{Method of Extracting Rdie and Cdie}

\subsection{Introduction}

As mentioned in chapter 1, large buffer chains are used in DDR controllers due to large physical size, which leads to large PSIJ. The mechanism of power supply noise is covered in Section 1.1.1 and a simplified PDN for an on-die circuit is shown in Figure 1.1 (redrawn as Figure 5.1 for convenience of reading). In today's PDN design, the PDN impedance is required to be less than the target impedance over the frequency range of interest to hold the noise voltage below the allowed ripple on the power supply. Therefore, low PDN impedance can prevent excessive voltage fluctuations and reduce power supply noise. The target impedance of a PDN to limit the voltage ripple on the power supply is given by [69],

$$
Z_{P D N}<\frac{V D D \times \% \text { Vripple }}{I_{\max }}
$$

where $V D D$ is the supply voltage, \%Vripple is the target percentage of the voltage ripple, and $I_{\max }$ is the maximum current of $I_{\text {switch }}$ in Figure 5.1. To achieve an optimized performance and cost PDN design, adding appropriate amount of on-die decoupling capacitors (ODC) is an 
effective and essential method. Over-design of ODC will improve PSIJ performance but it also leads to cost increase.

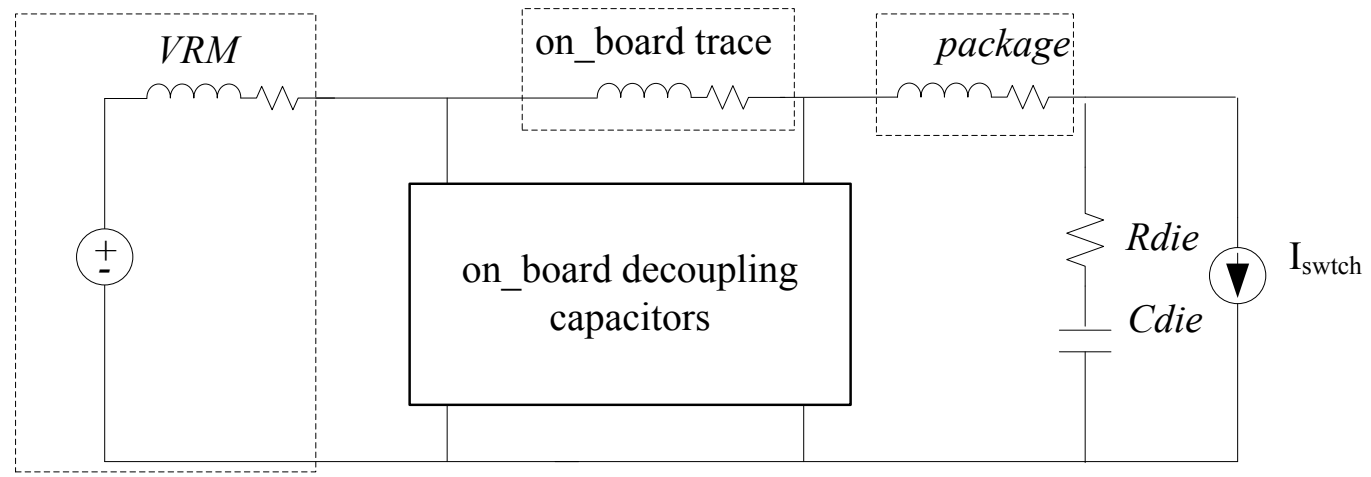

Figure 5.1: A simplified PDN for on-die circuits.

ODC comprises two components, the on-die power grid equivalent resistance (Rdie [31]) and the on-die power grid equivalent capacitance (Cdie [31]). In order to meet the jitter specification and keep the design cost as low as possible, Rdie and Cdie have become necessary parameters for memory controller IP vendors and customers.

Intrinsic on-die capacitance is mainly contributed by the metal power net, the non-switching gates and p-n diode junction diffusion (between the drain and substrate, the drain and well, and the well and substrate). The dominant contributor of intrinsic on-die capacitance is non-switching gate capacitance. The source of Rdie mainly includes power net resistance, transistor channel resistance, transistor gate resistance and the contact resistance of N-well and P-substrate. Figure 5.2 shows the physical location of the three types of 
intrinsic Cdie with an example of a cross section of a CMOS inverter [70].

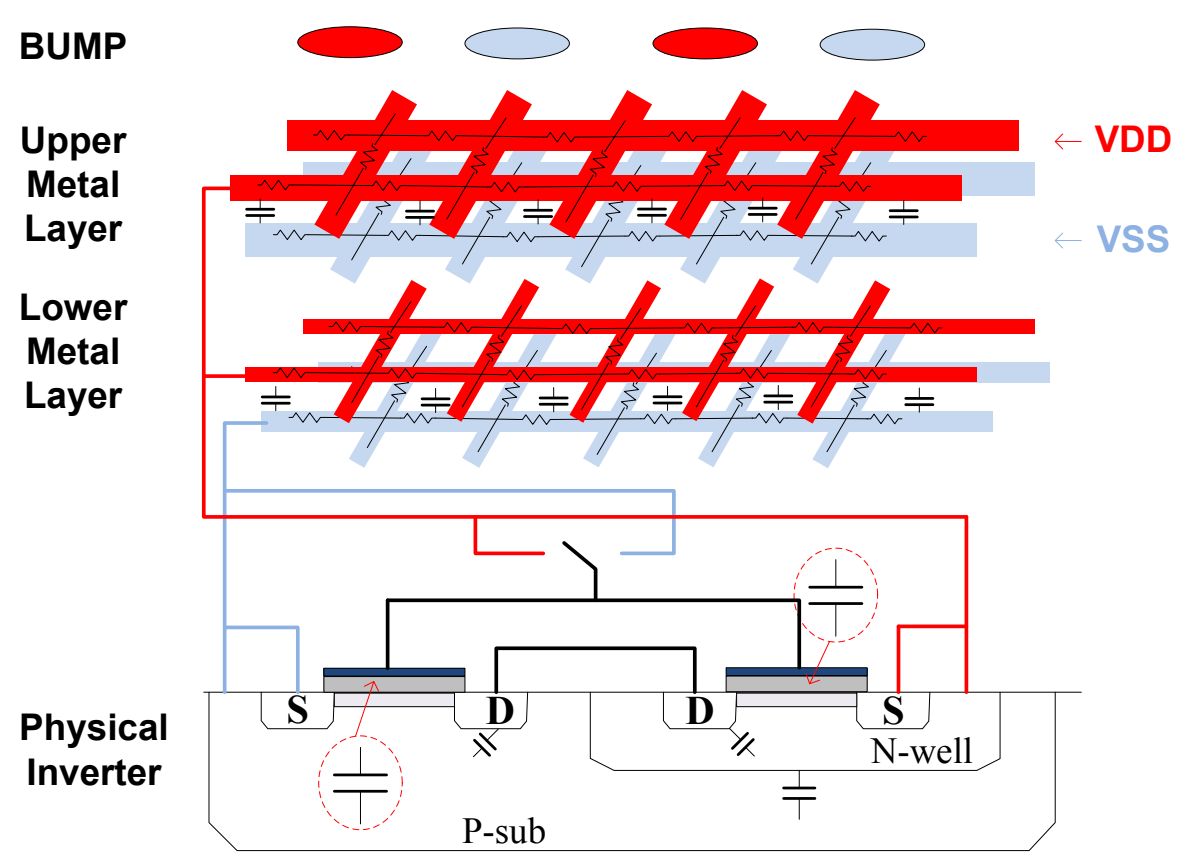

Figure 5.2: Cross section of a CMOS inverter and the physical locations of intrinsic ODC [71].

The dominant contributor of intrinsic on-die capacitance is non-switching gate capacitance distributed over the die. Millions or even billions of inverters can be easily found on many chips [72]. A transistor level CMOS inverter is shown in Figure 5.3(a). At any one time, one of the gates is on and the other is off. For example, when $V_{\text {in }}=0 V$, (a) is equivalent to (b) and when $V_{\text {in }}=V D D$, (a) is equivalent to (c). Obviously (b) and (c) function as the on-die decoupling capacitors. 


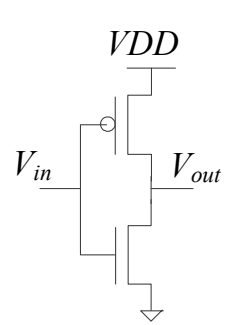

(a)

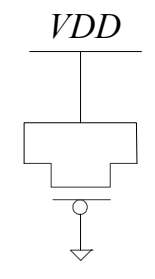

(b)

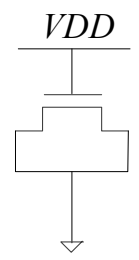

(c)

Figure 5.3: An inverter and its equivalent circuits. (a) Inverter; (b) Equivalent circuit of (a) as $V_{\text {in }}=0 V$; (c) Equivalent circuit of (a) as $V_{\text {in }}=V D D$.

Rdie and Cdie have been challenging in pre-silicon extraction and post-silicon measurement [71]. To extract Rdie and Cdie, commercial tools are available (e.g. Apache RedHawk [21], ANSYS Totem [73]). However, the expensive license fee prohibits wide application of these tools. In addition, all designers have SPICE-like simulators (e.g. Hspice, Spectre) which can be used to extract Rdie/Cdie for small circuit blocks with the highest accuracy (the accuracy is dependent on the accuracy of the model file which is from semiconductor foundries).

A method of extracting Rdie/Cdie for a large design is proposed in [31]. First, Rdie/Cdie for the subblocks is extracted without higher power/ground metal nets; second, the extracted Rdie/Cdie of the subblocks is connected at their corresponding locations to the higher power/ground metal nets, which is plotted in Figure 5.4. By shorting all VDD bumps together, shorting all VSS bumps together in a functional block, and then running AC simulation, a frequency domain Rdie/Cdie for the whole design is obtained. 


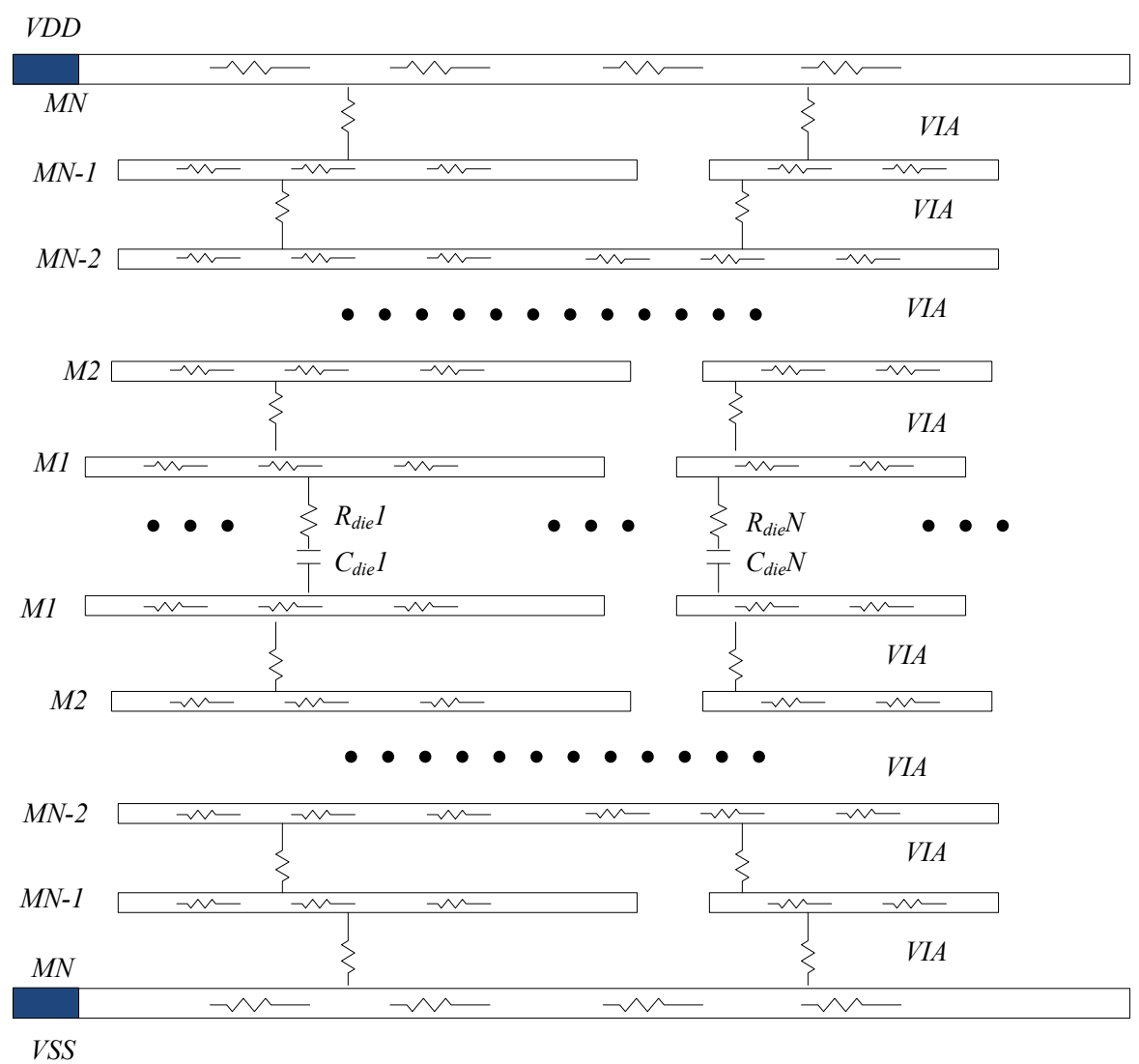

Figure 5.4: The entire extracted on-die power delivery network.

\subsection{The Effect of Rdie and Cdie}

The ODC and package lead inductance resonance generates the largest PDN impedance which typically is in the range between $50 \mathrm{MHz}$ and $500 \mathrm{MHz}$ [74]. The PDN impedance dominant peak can be lowered by decreasing the package inductance. The most simplified PDN system is shown in Figure 5.5, in which the board is simplified as a dead short. The relationship between PDN impedance and package inductance is shown in Figure 5.6. The 
relationship between PDN impedance and Cdie capacitance is shown in Figure 5.7. The relationship between PDN impedance and Rdie resistance is shown in Figure 5.8.

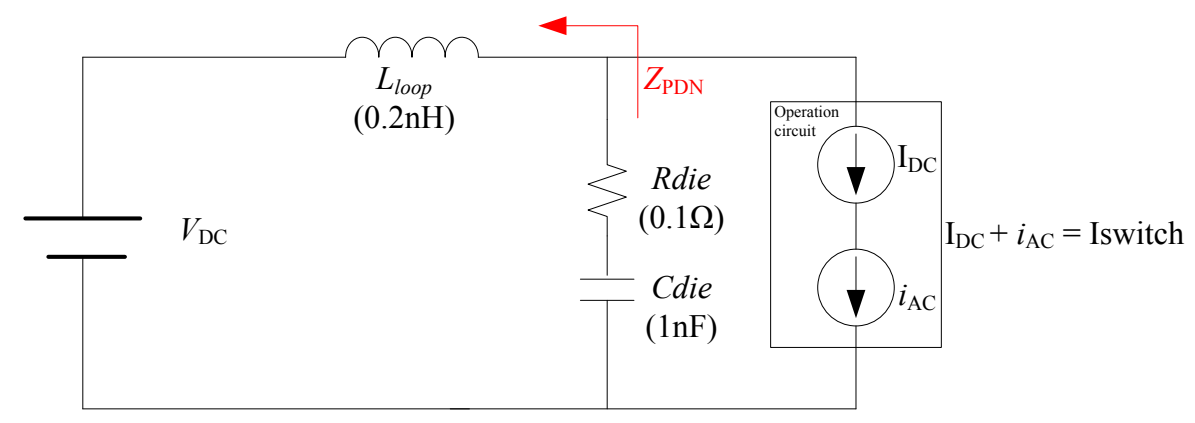

Figure 5.5: Simplified PDN system with dead short board assumption.

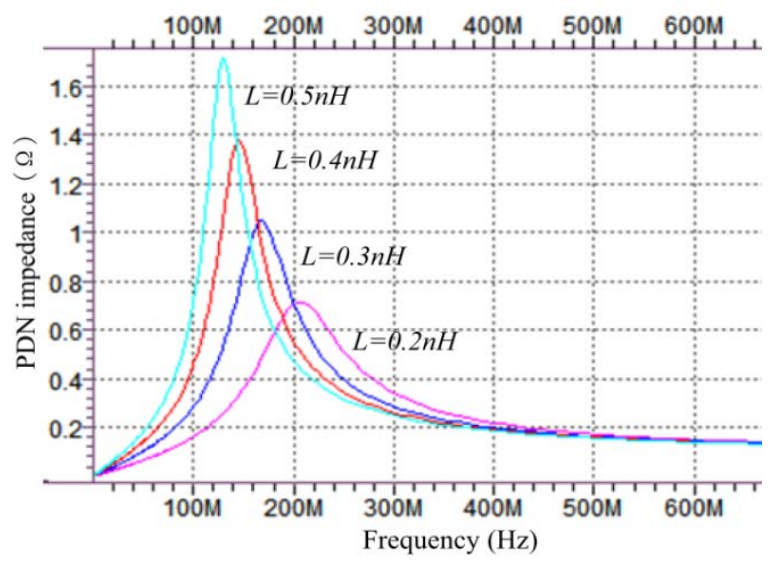

Figure 5.6: PDN impedance vs package inductance. 


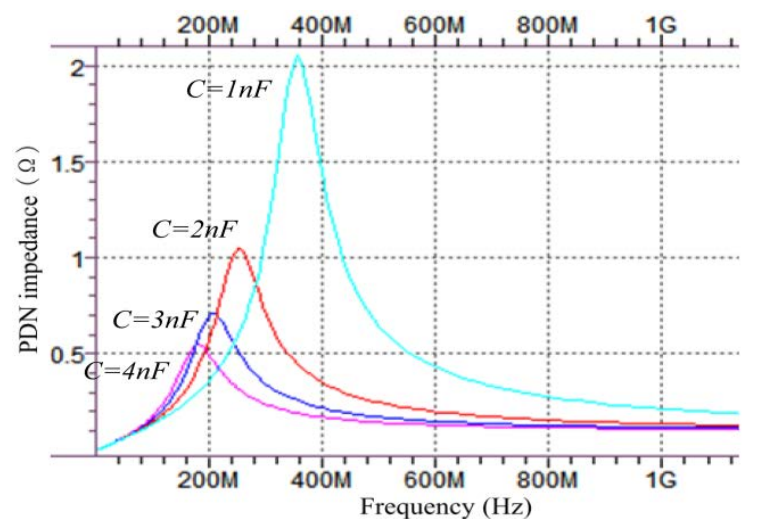

Figure 5.7: PDN impedance vs Cdie.

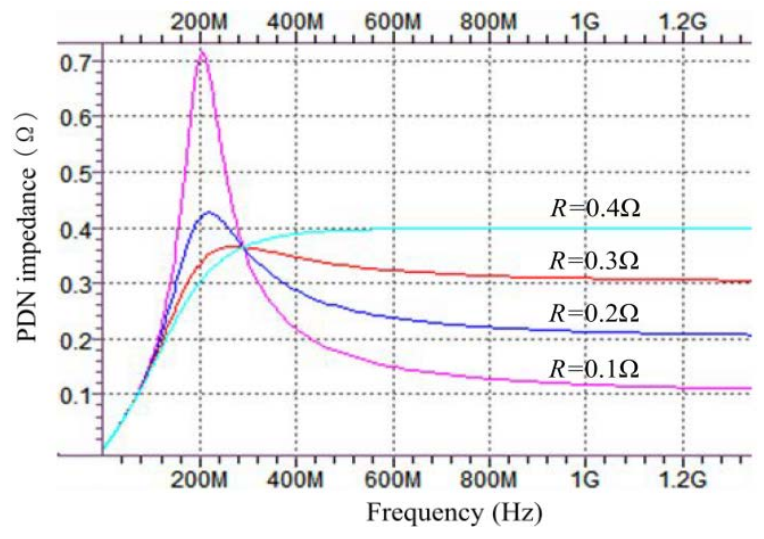

Figure 5.8: PDN impedance vs Rdie.

From Figure 5.6, 5.7 and 5.8, one can see that smaller package loop inductance and larger Cdie are essential for smaller resonant peak impedance. But Rdie value will be dependent on specific conditions. Larger Rdie will decrease the resonant peak but at the same time it will increase the PDN impedance at high frequency (compared to the peak), causing the increase of high frequency noise component. 


\subsection{Parasitic Models for IC (integrated circuit) Layout Extraction}

To extract Rdie and Cdie with a SPICE-like simulator, the IC layout extracted netlist is necessary. A parasitic extraction tool (e.g., Calibre xRC, StarRC) will be used to calculate parasitic resistance and capacitance in an IC layout and produce a simulatable netlist which is the extracted netlist.

For layout extractions, several main areas are evaluated and analyzed [75]. These are designed device extraction, connectivity extraction, parasitic in-line device (resistance) extraction, parasitic cross-coupled device (capacitance and inductance) extraction, and network reduction.

\subsubsection{Layout Extraction Evaluation Areas}

\subsubsection{Designed device extraction}

The tool will find the designed devices and determine their parameters, then remove them from the underlying artwork, if needed, so that what remains is the interconnect.

\subsubsection{Connectivity extraction}

The tool will find which combinations of the remaining geometry form nets, then assign a name to each net, after this, determine which nets are connected to which pins of the devices 
and which are externally visible.

\subsubsection{Resistance extraction}

Optionally, each net may be divided into one or more sub-nodes to account for the parasitic resistance of the net. This is a $2 \mathrm{D}$ problem. Along similar lines, the substrate may need to be subdivided as well. This is a 3D problem over a large area, and different techniques are used.

\subsubsection{Capacitance and inductance estimation}

Each piece of each net must have its capacitance and inductance estimated. Both capacitance and inductance may require both self and mutual terms.

\subsubsection{Reduction}

A straightforward extraction gives netlists that are too big for most practical uses. Reduction generates smaller netlists with very similar properties.

\subsubsection{Extraction Parasitic Model Options}

All the extraction tools provide extraction results with parasitic model options. The most basic parasitic models include lumped capacitance (C-only), distributed resistance only (R-only), lumped capacitance with coupled capacitors $(\mathrm{Cc})$, distributed resistance and capacitance $(\mathrm{RC})$ and distributed resistance and capacitance with coupling capacitor (RCc). 


\subsubsection{Lumped Capacitance (C-only) and lumped capacitance with coupled capacitors $(\mathbf{C c})$}

With lumped capacitance extraction, all capacitance for a net is modeled as one parasitic capacitor between the net and the substrate. Depending on the options (to select C-only or $\mathrm{Cc})$, the parasitic capacitor may include the effects of coupled capacitance to another net with the effect lumped to the ground. Resistance is not modeled [76]. Figure 5.9 shows a simplified layout of two instances with two nets. C-only and Cc extraction are shown in Figure 5.10 and Figure 5.11 respectively.

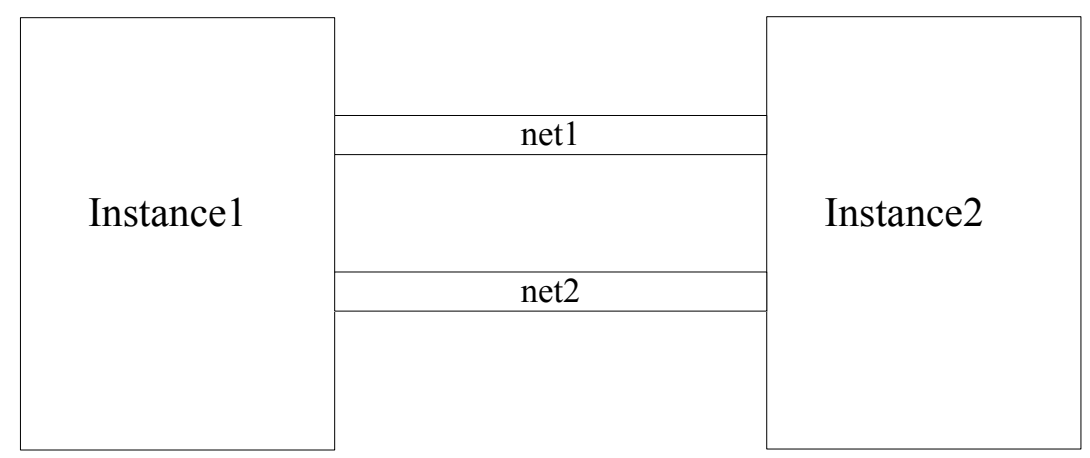

Figure 5.9: A simplified layout of two instances with two nets. 


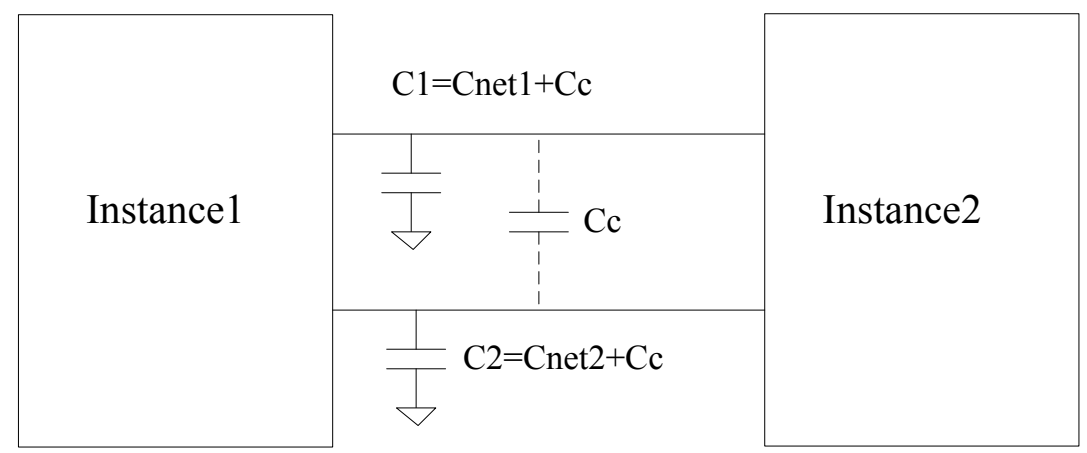

Figure 5.10: C-only extraction.

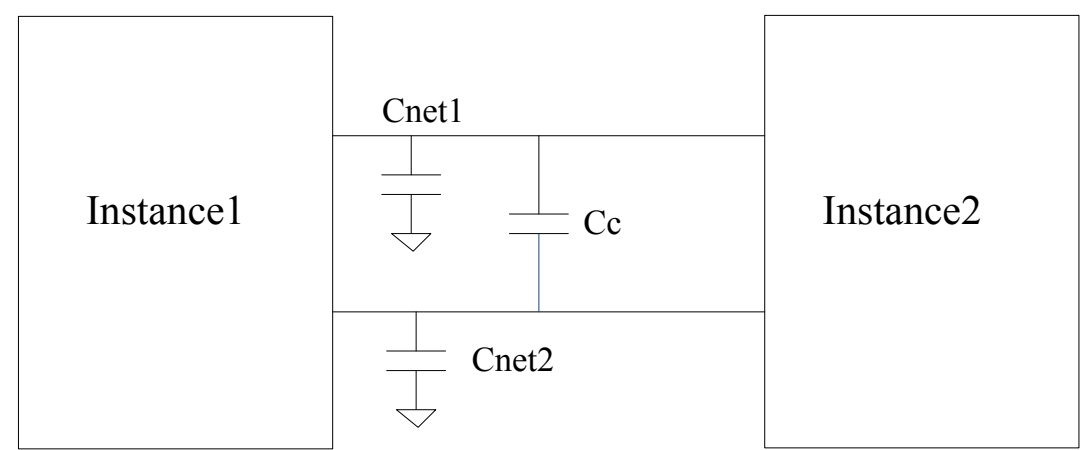

Figure 5.11: Cc extraction.

\subsubsection{Distributed resistance only (R-only)}

With a distributed resistance extraction, the parasitic resistance of the net is divided into segments representing geometric regions [76]. Capacitance is not modeled. Figure 5.12 shows a simplified layout example with the equivalent distributed resistance extraction model. The net 1 and net 2 are respectively separated into 3 segments and the extracted resistance is shown in Figure 5.13. 


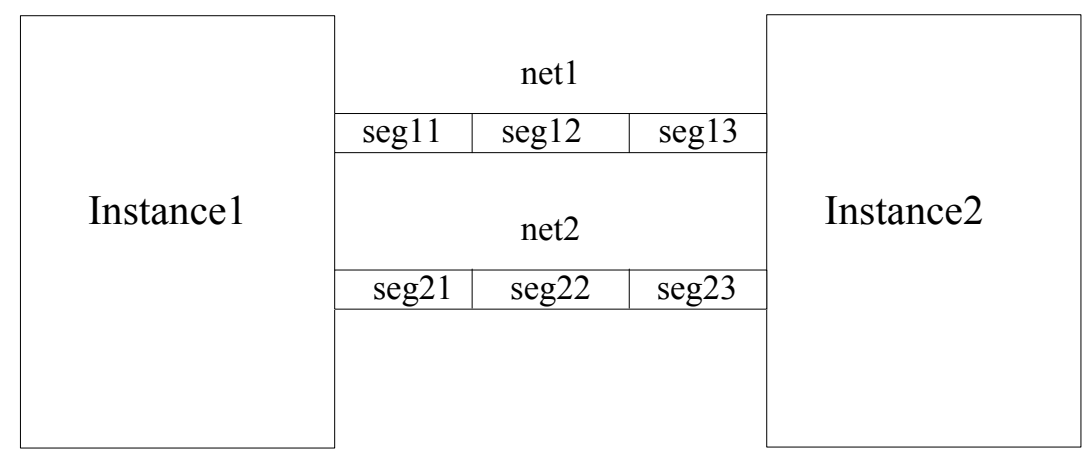

Figure 5.12: Net has been divided into $\mathrm{N}$ segments for parasitic resistance extraction.

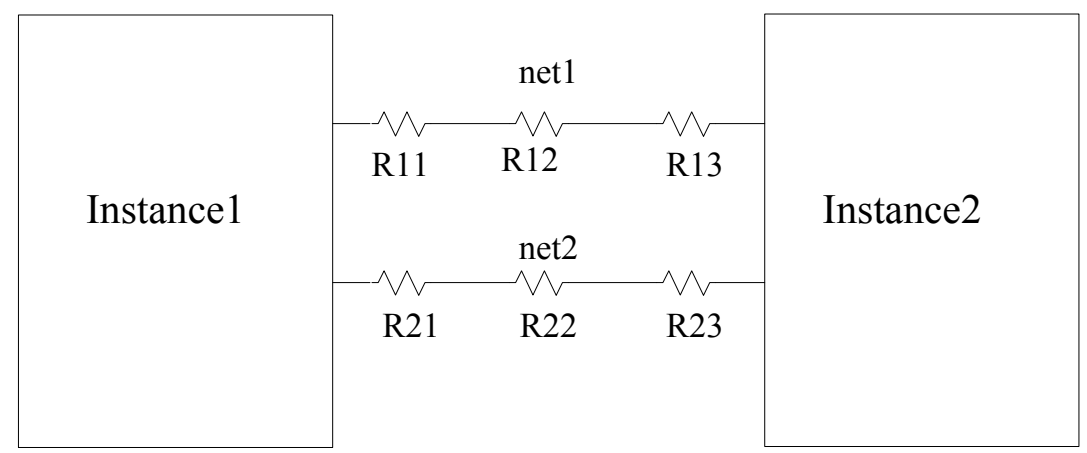

Figure 5.13: R-only extraction.

\subsubsection{Distributed resistance and capacitance (RC) and distributed resistance and capacitance with coupling capacitor $(\mathrm{RCc})$}

With a distributed resistance and capacitance extraction, the parasitic resistance of the net is broken into segments representing geometric regions. The parasitic capacitance is likewise divided into "local" segments going to the substrate, including the effect of coupled capacitance [76]. Figure 5.14 shows an example of RC extraction. The RCc extraction is 
shown in Figure 5.15.

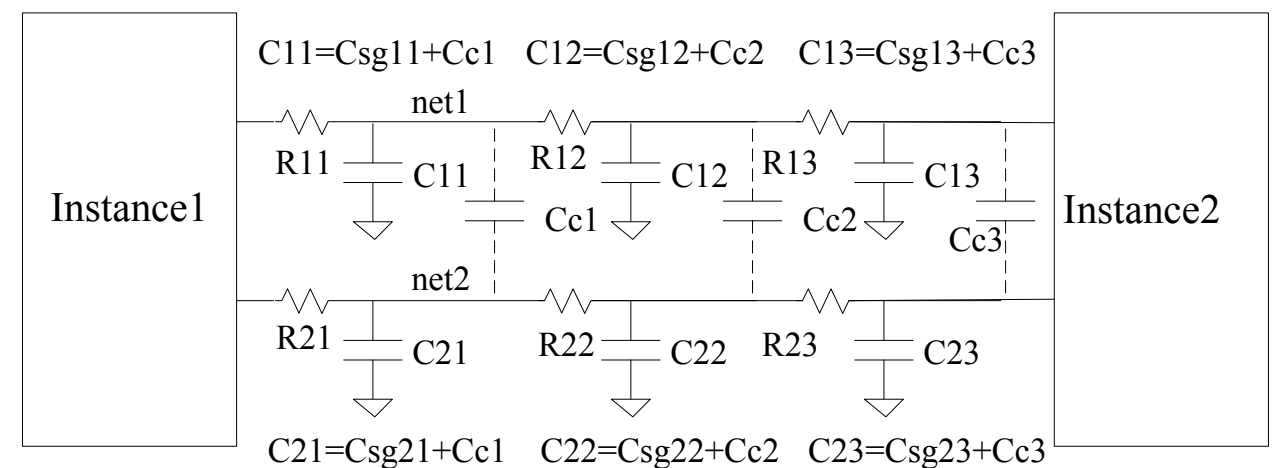

Figure 5.14: RC extraction.

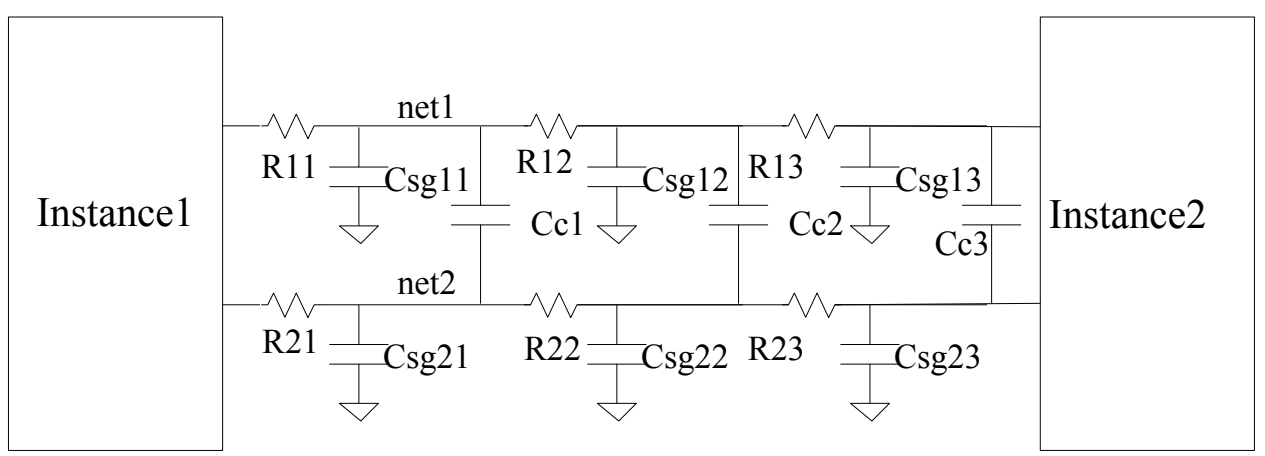

Figure 5.15: RCc extraction.

\subsection{Methodologies of Extracting Rdie and Cdie}

In the early days, the supply voltage was relatively high and the DDR interface speed was relatively low, only the capacitance of ODC was considered for the PDN design. The capacitance of ODC is extracted with time domain simulation from C-only or Cc layout 
extraction netlist. As the power supply voltage scales down to lower than 1 volt, and data rate goes up to $3.2 \mathrm{Gbps}$ and higher, both Rdie and Cdie need to be considered because C-only method causes overestimation of on-die decoupling capacitor. A SPICE-like simulator (Hspice or Spectre) avoids extra costs on commercial tools and thus is the best choice for most IP vendors and circuit designers to extract Rdie and Cdie.

It is necessary to have a layout extracted netlist when a SPICE-like simulator is used to extract Rdie/Cdie. The RC option is a relatively better choice when compared with other extraction options (C-only, Cc, R-only, and RCc). The C-only, Cc and R-only operations provide relatively low accurate models for Rdie/Cdie extraction. The RCc operation provides a more accurate model than the RC operation. However, the dominant contributor of intrinsic on-die capacitance is non-switching gate capacitance in the RCc operation; the coupling capacitance between the nets is so small that it can be ignored. In addition, the RC extracted netlist is much smaller than the RCc netlist. When extracting Rdie/Cdie with the smaller RC extracted netlist, the simulation time can be greatly reduced.

To accurately extract Rdie and Cdie, an AC analysis method is the best choice. Two AC analysis methods have been widely used in industry: unit voltage method and unit current method, which is discussed in the section of 5.4.2. 


\subsubsection{Methodology of Extracting Cdie-only}

During the days of DDR2 and the early stage of DDR3, SI engineers only cared about the capacitance value of Cdie. The capacitance of Cdie of a whole system is calculated as the sum of the capacitance of each subcircuit block. The capacitance of each subcircuit block is extracted from the $\mathrm{Cc}$ extracted netlist. A typical methodology can be found in Figure 5.16.

In the red box of Figure 5.16(a) is the extracted $\mathrm{Cc}$ netlist. Because the parasitic resistance is not modeled, all the capacitance is in parallel between power supply VDD and ground net VSS. Resistor $R_{\text {leak }}$ is used to model the leakage current. If there is no leakage current, the value of $R_{\text {leak }}$ will be infinity. To extract the total capacitance, a ramp supply voltage between VDD- $\Delta \mathrm{V} / 2$ and $\mathrm{VDD}+\Delta \mathrm{V} / 2$ with time duration of $\Delta \mathrm{t}$ is provided. The total current flowing into or out of the power supply node is measured. A typical waveform of the current can be found in Figure 5.16(c). As the supply voltage changes from VDD- $\Delta$ V/2 to $\mathrm{VDD}+\Delta \mathrm{V} / 2$, the current flowing into the circuit presents a step waveform, this is because capacitors block the DC signals, so the DC current only flows through resistor $\mathrm{R}_{\text {leak. }}$. A ramp voltage is an AC signal, so there will be current flowing through both the capacitors and $\mathrm{R}_{\text {leak }}$. The value of the total current can be calculated with this expression,

$$
I_{\text {total }} \approx C_{\text {total }} \frac{d V D D}{d t}+\frac{V D D}{R_{\text {leak }}}=C_{\text {total }} \frac{\Delta V}{\Delta t}+\frac{V D D}{R_{\text {leak }}}=\Delta I+\frac{V D D}{R_{\text {leak }}}
$$

where $\mathrm{C}_{\text {total }}$ is Cdie and can be obtained as, 


$$
C_{\text {die }}=C_{\text {total }}=\Delta I /\left(\frac{\Delta V}{\Delta t}\right)
$$

Normally $\Delta \mathrm{V}$ is much less than VDD. This is because supply voltage variation normally is very small to ensure that the circuit operates correctly.

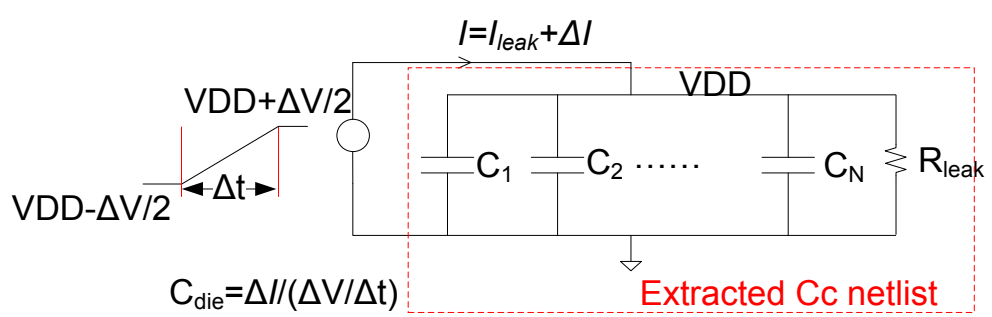

(a)

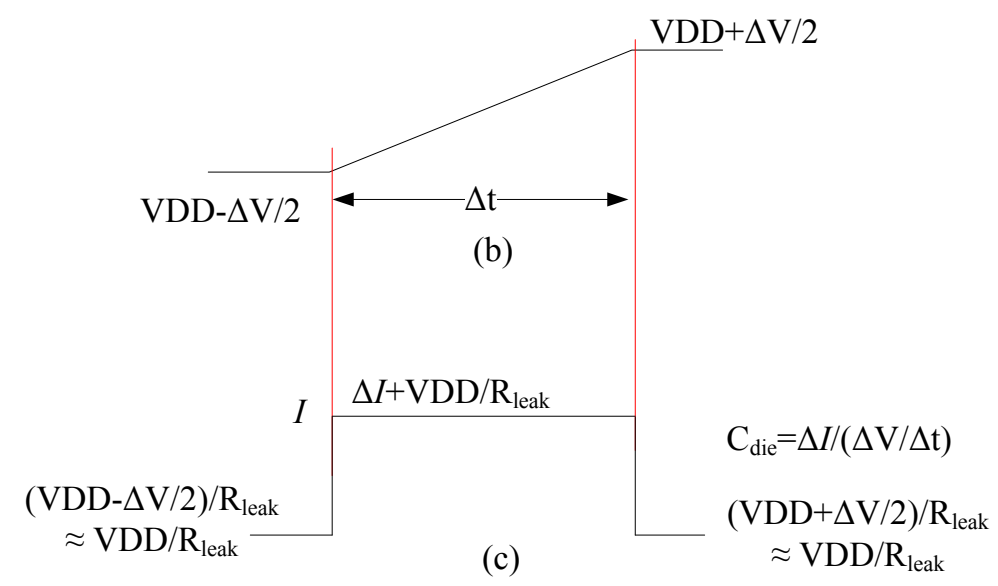

Figure 5.16: Cdie-only extraction method. (a) Testbench of Cdie-only extraction; (b) Ramp voltage added to power supply; and (c) Current flowing into node VDD due to the added ramp voltage shown in (b).

\subsubsection{Methodologies of Extracting Both Rdie and Cdie}

Two main AC analysis methods have been used in industry: (A) the unit voltage method, and (B) the unit current method. 


\subsubsection{Method A: Unit voltage AC analysis method}

The principle is shown in Figure 5.17. The extracted netlist and equivalent Rdie and Cdie are shown in the dashed line boxes. A IV AC source provides the stimulus. As a result, an AC current $\boldsymbol{I}$ is generated and the real and imaginary components of $\boldsymbol{I}, \boldsymbol{i}_{r}$ and $\boldsymbol{i}_{\boldsymbol{i}}$ respectively, can be found.
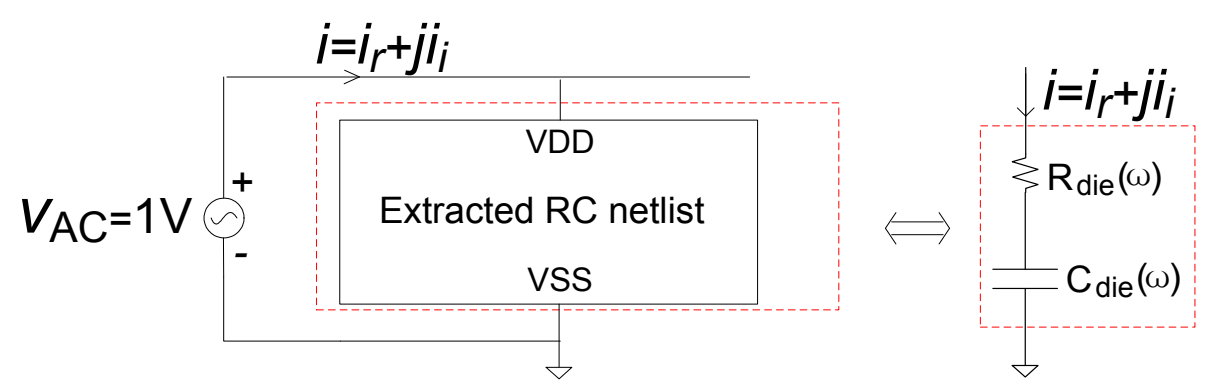

Figure 5.17: Method A: Unit voltage AC analysis method.

According to Ohm's law, one can obtain the following equation,

$$
\frac{1}{R_{d i e}+\frac{1}{j \omega C_{d i e}}}=i_{r}+j i_{i}
$$

From the real part and imaginary part in (5.4), one can get

$$
\begin{aligned}
& i_{r}=\frac{R_{d i e}}{R_{d i e}^{2}+\frac{1}{\omega^{2} C_{d i e}^{2}}} \\
& i_{i}=\frac{\frac{1}{\omega C_{d i e}}}{R_{d i e}^{2}+\frac{1}{\omega^{2} C_{d i e}^{2}}}
\end{aligned}
$$


Rdie and Cdie can be derived from (5.5) and (5.6)

$$
\begin{gathered}
R_{d i e}=\frac{1}{i_{r}\left(1+\frac{i_{i}^{2}}{i_{r}^{2}}\right)} \\
C_{d i e}=\frac{1}{2 \pi f i_{i}}\left(i_{r}^{2}+i_{i}^{2}\right)
\end{gathered}
$$

\subsubsection{Method B: Unit current AC analysis method}

The principle is shown in Figure 5.18. The stimulus is a $1 \mathrm{~A}$ AC current. The real and imaginary components of the simulated $\mathrm{AC}$ voltage $\boldsymbol{v}, \boldsymbol{v}_{\boldsymbol{r}}$ and $\boldsymbol{v}_{\boldsymbol{i}}$, respectively, can be determined from the simulation.

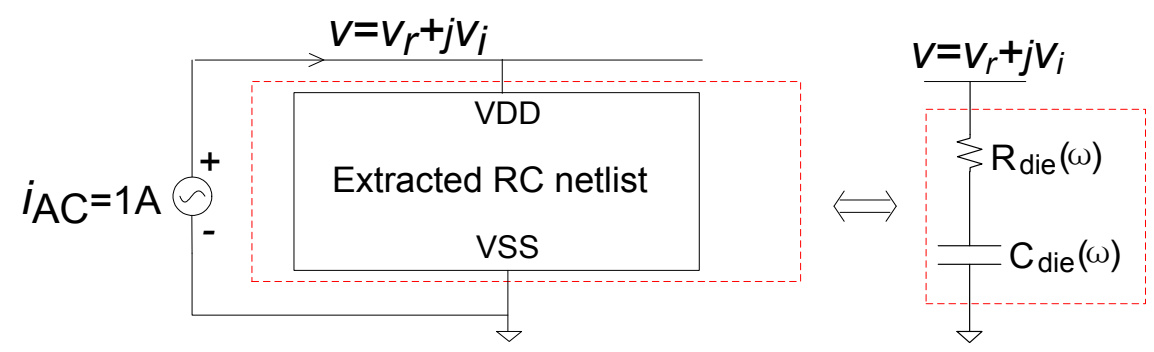

Figure 5.18: Method B: Unit current AC analysis method.

Similar to method A, according to Ohm's law, one can obtain the following equation,

$$
1 \cdot\left(R_{d i e}+\frac{1}{j \omega C_{d i e}}\right)=v_{r}-j v_{i}
$$

The negative sign can be seen in the equation above because the voltage applied to Cdie lags the current by $90^{\circ}$. From the real part and imaginary part in (5.9), one can get 


$$
\begin{gathered}
R_{d i e}=v_{r} \\
\frac{1}{j \omega C_{d i e}}=-j v_{i} \Rightarrow C_{d i e}=\frac{1}{2 \pi f v_{i}}
\end{gathered}
$$

As an example, through method $\mathrm{B}$, the extracted Rdie and Cdie from a $\mathrm{RC}$ extraction netlist of a circuit named chipA were computed for various source frequencies and are shown in Table 5.1. The values of Rdie and Cdie change with the source frequency and consequently the simple lumped frequency domain narrowband R-C model in Figures 5.17 and 5.18 cannot be directly used for time domain transient simulation to plot eye-diagrams for the jitter estimation (which is widely used by SI engineers).

Table 5.1 Rdie and Cdie vs. frequency.

\begin{tabular}{|c|c|c|c|}
\hline Num, $i$ & Frequency $f_{\mathrm{i}}(\mathrm{Hz})$ & Rdie $(\Omega)$ & Cdie $(\mathrm{F})$ \\
\hline 1 & $3.00 \mathrm{e} 7$ & $5.83 \mathrm{e}-01$ & $1.26 \mathrm{e}-09$ \\
\hline 2 & $2.50 \mathrm{e} 8$ & $4.95 \mathrm{e}-01$ & $9.44 \mathrm{e}-10$ \\
\hline 3 & $1.26 \mathrm{e} 9$ & $2.12 \mathrm{e}-01$ & $4.40 \mathrm{e}-10$ \\
\hline 4 & $3.75 \mathrm{e} 9$ & $1.08 \mathrm{e}-01$ & $2.95 \mathrm{e}-10$ \\
\hline
\end{tabular}

\subsection{Proposed Broadband Equivalent Circuit of Frequency}

\section{Domain Rdie and Cdie}

To overcome the limitation of the lumped frequency domain Rdie and Cdie model for time domain transient simulation for jitter analysis, an approximate broadband equivalent circuit, 
which comprises $\mathrm{N}$ series-RC subcircuits placed in parallel, is proposed in this dissertation. The principle is shown in Figure 5.19.

Once the Rdie and Cdie vs. frequency table (e.g. Table 5.1) is obtained, the resulting $\mathrm{N}$ narrowband lumped circuits can be used to construct a broadband approximate model corresponding to the original extracted netlist. The broadband model is intended to match the narrowband lumped models at each of the target frequencies. The following cases show how the broadband equivalent circuit works.

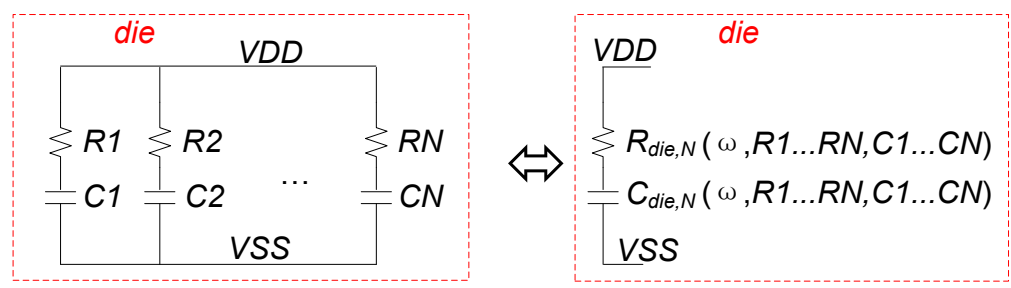

Figure 5.19: Parallel N series-RC-pairs circuit.

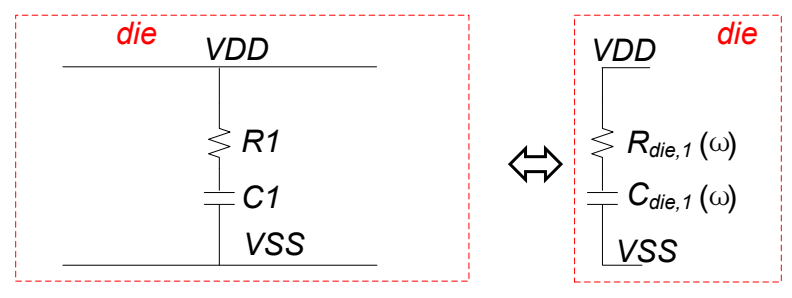

Figure 5.20: Parallel N=1 series-RC-pairs circuit.

Case 1: $N=1$ is chosen. The parallel $\mathrm{N}$ series-RC-pairs circuit is simplified as shown in

Figure 5.20. Then $R_{\mathrm{die}}(\omega)=R_{\mathrm{die}, 1}(\omega)=\mathrm{R} 1$ and $C_{\mathrm{die}}(\omega)=C_{\mathrm{die}, 1}(\omega)=\mathrm{C} 1$, where $\mathrm{R} 1$ and $\mathrm{C} 1$ can 
be any pair of Rdie/Cdie in Table 5.1. If $i=3$ is chosen, $R_{\mathrm{die}}(\omega)=R_{\mathrm{die}, 1}(\omega)=2.12 \mathrm{e}-01 \Omega$ and $C_{\mathrm{die}}(\omega)=C_{\mathrm{die}, 1}(\omega)=4.40 \mathrm{e}-10 \mathrm{~F}$, which means that only at one frequency point, $1.26 \mathrm{e} 9 \mathrm{~Hz}$, the equivalent broadband circuit is accurate.

Case 2: $N=2$ is chosen. The parallel N series-RC-pairs circuit is simplified as shown in Figure 5.21. Then $R_{\mathrm{die}, 2}(\omega)$ and $C_{\mathrm{die}, 2}(\omega)$ can be strictly derived from R1/C1 and $\mathrm{R} 2 / \mathrm{C} 2$,

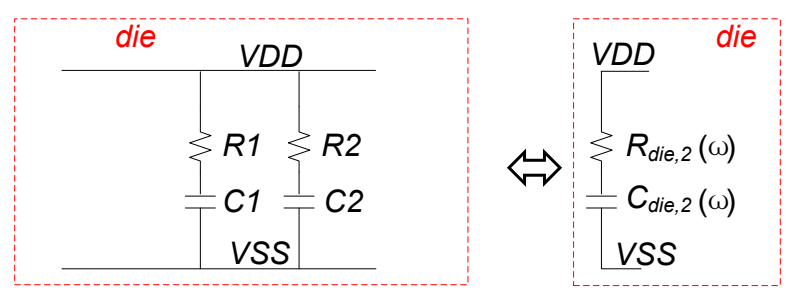

Figure 5.21: Parallel N=2 series-RC-pairs circuit.

$$
\begin{aligned}
& Z_{d i e, 2}(\omega)=R_{d i e, 2}(\omega)+\frac{1}{s C_{d i e, 2}(\omega)}=\left(R_{1}+\frac{1}{s C_{1}}\right) \|\left(R_{2}+\frac{1}{s C_{2}}\right) \\
& =\frac{\left[\left(R_{1} R_{2}+\frac{1}{s^{2} C_{1} C_{2}}\right)\left(R_{1}+R_{2}\right)-\frac{1}{s^{2}}\left(\frac{R_{1}}{C_{2}}+\frac{R_{2}}{C_{1}}\right)\left(\frac{1}{C_{1}}+\frac{1}{C_{2}}\right)\right]+\frac{1}{s}\left[\left(\frac{R_{1}}{C_{2}}+\frac{R_{2}}{C_{1}}\right)\left(R_{1}+R_{2}\right)-\left(R_{1} R_{2}+\frac{1}{s^{2} C_{1} C_{2}}\right)\left(\frac{1}{C_{1}}+\frac{1}{C_{2}}\right)\right]}{\left(R_{1}+R_{2}\right)^{2}-\frac{1}{s^{2}}\left(\frac{1}{C_{1}}+\frac{1}{C_{2}}\right)^{2}} \\
& R_{d i e, 2}(\omega)=\operatorname{Re}\left\{Z_{d i e, 2}(\omega)\right\}=\frac{\left(R_{1} R_{2} C_{1}^{2} C_{2}^{2} \omega^{2}-C_{1} C_{2}\right)\left(R_{1}+R_{2}\right)+\left(R_{1} C_{1}+R_{2} C_{2}\right)\left(C_{1}+C_{2}\right)}{\omega^{2}\left(R_{1}+R_{2}\right)^{2} C_{1}^{2} C_{2}^{2}+\left(C_{1}+C_{2}\right)^{2}} \\
& \operatorname{Im}\left\{Z_{d i e, 2}(\omega)\right\}=\frac{1}{s C_{d i e, 2}(\omega)} \\
& C_{d i e, 2}(\omega)=\frac{\omega^{2}\left(R_{1}+R_{2}\right)^{2} C_{1}^{2} C_{2}^{2}+\left(C_{1}+C_{2}\right)^{2}}{\omega^{2} C_{1} C_{2}\left(R_{1} C_{1}+R_{2} C_{2}\right)\left(R_{1}+R_{2}\right)-\left(\omega^{2} R_{1} R_{2} C_{1} C_{2}-1\right)\left(C_{1}+C_{2}\right)}
\end{aligned}
$$

After choosing any two pairs of Rdie/Cdie in Table 5.1, one can plug the chosen 
Rdie/Cdie value into (5.12) and (5.13) and solve the four simultaneous equations to obtain Rdie1/Cdie1 and Rdie2/Cdie2. The parallel 2 series-RC-pairs equivalent circuit shown in Figure 5.21 is only accurate at two chosen frequency points.

Case 3: $N=N$ is chosen. Taking advantage of $\mathrm{N}=2$ results, (5.12) and (5.13), one can recursively get $\mathrm{N}=3, R_{\mathrm{die}, 3}(\omega)$ and $C_{\mathrm{die}, 3}(\omega)$ expressed as,

$$
\left\{\begin{array}{l}
R_{d i e, 3}(\omega)=\frac{\left(R_{d i e, 2} R_{3} C_{d i e, 2}^{2} C_{3}^{2} \omega^{2}-C_{d i e, 2} C_{3}\right)\left(R_{d i e, 2}+R_{3}\right)+\left(R_{d i e, 2} C_{3}+R_{3} C_{3}\right)\left(C_{d i e, 2}+C_{3}\right)}{\omega^{2}\left(R_{d i e, 2}+R_{3}\right)^{2} C_{d i e, 2}^{2} C_{3}^{2}+\left(C_{d i e, 2}+C_{3}\right)^{2}} \\
C_{d i e, 3}=\frac{\omega^{2}\left(R_{d i e, 2}+R_{3}\right)^{2} C_{d i e, 2}^{2} C_{3}^{2}+\left(C_{d i e, 2}+C_{3}\right)^{2}}{\omega^{2} C_{d i e, 2} C_{3}\left(R_{d i e, 2} C_{d i e, 2}+R_{3} C_{3}\right)\left(R_{d i e, 2}+R_{3}\right)-\left(\omega^{2} R_{d i e, 2} R_{3} C_{d i e, 2} C_{3}-1\right)\left(C_{d i e, 2}+C_{3}\right)}
\end{array}\right.
$$

By the same means, $R_{\mathrm{die}, \mathrm{N}}(\omega)$ and $C_{\mathrm{die}, \mathrm{N}}(\omega)$ can be given as,

$$
\left\{\begin{array}{l}
R_{d i e, N}(\omega)=\frac{\left(R_{d i e, N-1} R_{N} C_{d i e, N-1}^{2} C_{N}^{2} \omega^{2}-C_{d i e, N-1} C_{N}\right)\left(R_{d i e, N-1}+R_{N}\right)+\left(R_{d i e, N-1} C_{N}+R_{N} C_{N}\right)\left(C_{d i e, N-1}+C_{N}\right)}{\omega^{2}\left(R_{d i e e}+R_{3}\right)^{2} C_{d i e 2}^{2} C_{3}^{2}+\left(C_{d i e 2}+C_{3}\right)^{2}} \\
C_{d i e, N}=\frac{\omega^{2}\left(R_{d i e, N-1}+R_{N}\right)^{2} C_{d i e, N-1}^{2} C_{N}^{2}+\left(C_{d i e, N-1}+C_{N}\right)^{2}}{\omega^{2} C_{d i e, N-1} C_{N}\left(R_{d i e, N-1} C_{d i e, N-1}+R_{N} C_{N}\right)\left(R_{d i e, N-1}+R_{N}\right)-\left(\omega^{2} R_{d i e, N-1} R_{N} C_{d i e, N-1} C_{N}-1\right)\left(C_{d i e, N-1}+C_{N}\right)}
\end{array}\right.
$$

The collection of frequency-specific narrowband lumped circuits is modeled by using the N-branch broadband circuit shown in Figure 5.19. Knowing that the equivalent models shown in Figure 5.19 correspond at the $\mathrm{N}$ modeled frequencies, one can create $2 \mathrm{~N}$ simultaneous equations of Rdie and Cdie at the $\mathrm{N}$ frequency points, and then solve the equation set. The $2 \mathrm{~N}$ equations can be built recursively with (5.15).

The left-hand side of (5.15) can be replaced with the N Rdie and N Cdie values in Table 5.1 and the $\omega$ in the right-hand side of (5.15) can be replaced with the frequency 
corresponding to the value of $\mathrm{N}$.

Optimization algorithms may be used to find the broadband circuit component values.

Here the weighted least square optimization algorithm is chosen. The objective function is expressed as,

$$
F(e(i))=\sum_{i=1}^{N}\left(\begin{array}{l}
\left(R_{d i e, N_{-} \omega i}-\frac{X+Y}{Z}\right)^{2} / R_{d i e, N_{-} \omega i}^{2}+ \\
\left(C_{\text {dieN_ } \_i}-\frac{U}{V-W}\right)^{2} / C_{d i e, N_{-} \omega i}^{2}
\end{array}\right)
$$

where

$$
\left\{\begin{array}{l}
X=\left(R_{d i e, N-1} R_{N} C_{d i e, N-1}^{2} C_{N}^{2} \omega_{i}^{2}-C_{d i e, N-1} C_{N}\right)\left(R_{d i e, N-1}+R_{N}\right) \\
Y=\left(R_{d i e, N-1} C_{N}+R_{N} C_{N}\right)\left(C_{d i e, N-1}+C_{N}\right) \\
Z=U=\omega_{i}^{2}\left(R_{d i e}+R_{3}\right)^{2} C_{d i e}^{2} C_{3}^{2}+\left(C_{d i e}+C_{3}\right)^{2} \\
V=\omega_{i}^{2} C_{d i e, N-1} C_{N}\left(R_{d i e, N-1} C_{d i e, N-1}+R_{N} C_{N}\right)\left(R_{d i e, N-1}+R_{N}\right) \\
W=\left(\omega_{i}^{2} R_{d i e, N-1} R_{N} C_{d i e, N-1} C_{N}-1\right)\left(C_{d i e, N-1}+C_{N}\right)
\end{array},\right.
$$

in which $R_{\mathrm{die}, \mathrm{N} \_\omega \mathrm{i}}$ and $C_{\mathrm{die}, \mathrm{N} \_\omega \mathrm{i}}$ are the Rdie and Cdie values in Table 1 at frequency $f_{\mathrm{i}}$ $\left(\omega_{\mathrm{i}}=2 \pi f_{\mathrm{i}}\right)$. The residual items with $R_{\mathrm{die}, \mathrm{N} \_\omega \mathrm{i}}$ and $C_{\mathrm{die}, \mathrm{N} \_\omega \mathrm{i}}$ in $(5.16)$ are weighted with $1 / R_{\mathrm{die}, \mathrm{N} \_\omega \mathrm{i}}$ and $1 / C_{\text {die, } \_\_i \mathrm{i}}$ (or normalized by the values of $R_{\mathrm{die}, \mathrm{N} \_\omega \mathrm{i}}$ and $C_{\mathrm{die}, \mathrm{N} \_\omega \mathrm{i}}$ ) respectively, which makes the contribution to the objective function equal for any squared residual item with $R_{\mathrm{die}, \mathrm{N} \_\omega i}$ or $C_{\mathrm{die}, \mathrm{N} \_\omega i}$. Without the weights, the contribution of residual items with $C_{\mathrm{die}, \mathrm{N} \_\omega i}$ will be totally negligible because $R_{\mathrm{die}}(\omega)$ is normally larger than $10^{-3} \Omega$ and $\mathrm{C}_{\mathrm{die}}(\omega)$ normally is between the range of tens of $\mathrm{nF}$ and several $\mathrm{pF}$. 


\subsection{Validation of the Proposed Broadband Equivalent Circuit}

To validate the proposed method and equation (5.16), one can take $\mathrm{N}=4$ and create eight simultaneous equations to convert the narrowband lumped Rdie and Cdie in Table 5.1 into an approximate broadband equivalent circuit with four series_RC subcircuits placed in parallel. One can choose the frequencies reported in Table 5.1, along with the corresponding lumped

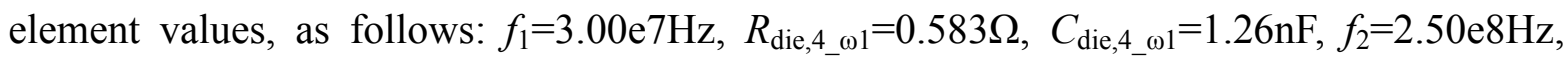
$R_{\mathrm{die}, 4 \_\omega 2}=0.495 \Omega, \quad C_{\mathrm{die}, 4 \_\omega 2}=944 \mathrm{pF}, \quad f_{3}=1.26 \mathrm{e} 9 \mathrm{~Hz}, \quad R_{\mathrm{die}, 4 \_\omega 3}=0.212 \Omega, \quad C_{\mathrm{die}, 4 \_\omega 3}=440 \mathrm{pF}$, $f_{4}=3.75 \mathrm{e} 9 \mathrm{~Hz}, R_{\text {die, } 4 \_\omega 4}=0.108 \Omega$ and $C_{\text {die, } 4 \_\omega 4}=295 \mathrm{pF}$. These element values are plugged into (5.16) to find the approximate broadband equivalent circuit model as $\mathrm{R} 1=0.089 \Omega, \mathrm{C} 1=188 \mathrm{pF}$, $\mathrm{R} 2=2.55 \Omega$ and $\mathrm{C} 2=466 \mathrm{pF}, \mathrm{R} 3=0.785 \Omega, \mathrm{C} 3=100 \mathrm{pF}, \mathrm{R} 4=1.40 \Omega$ and $\mathrm{C} 4=495 \mathrm{pF}$. Figure 5.22 shows the approximate broadband equivalent circuit.

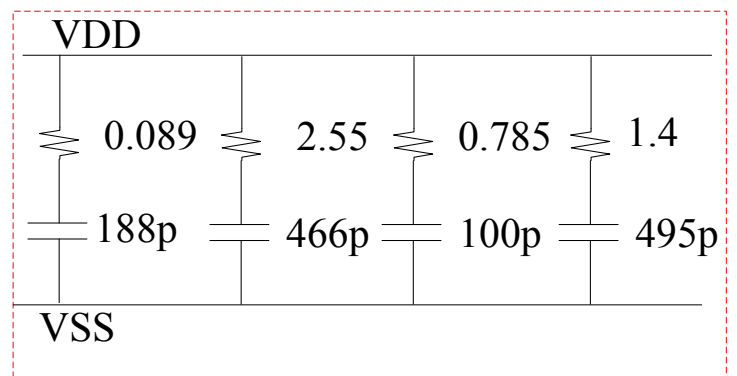

Figure 5.22: The approximate broadband equivalent circuit of the frequency domain Rdie/Cdie in Table 5.1. 
The simulation results using a full RC extracted netlist and the approximate broadband equivalent circuit are shown in Figure 5.23. The curves with " $\mathrm{x}$ " marks contain the results from the extracted netlist and the curves with "o" marks are from the approximate broadband equivalent circuit. Method B is used to derive $\operatorname{Rdie}(\omega)$ and $\operatorname{Cdie}(\omega)$ from each simulation. Figure 5.23(a) shows the results of Rdie plotted based on (5.10). Cdie results are shown in Figure 5.23(b), which is based on (5.11). The total impedance $\mathrm{Z}$ of equivalent series Rdie and Cdie is plotted in Figure 5.23(c). The Rdie, Cdie and total impedance Z error percentage of the approximate broadband equivalent circuit to the extracted results are shown in Figure 5.24. Figure 5.24(a) shows the Rdie error percentage and the maximum number is 5.23 during the frequency range of $30 \mathrm{MHz}$ to $3.75 \mathrm{GHz}$. The maximum Cdie error percentage is 5.82 shown in Figure 5.24(b) and the maximum total impedance $\mathrm{Z}$ error percentage is 2.973 plotted in Figure 5.24(c). It can be seen that all the Rdie, Cdie and total impedance of the approximate model match the original results very well from the extracted netlist over the frequency range of interest. To further improve the accuracy and frequency range, one can use more RC branches in the broadband model, and include further values of Rdie $(\omega)$ and Cdie $(\omega)$ corresponding to a wider frequency range. 


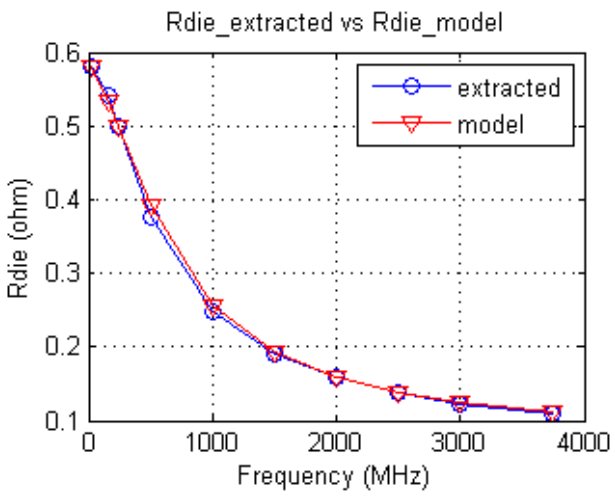

(a)

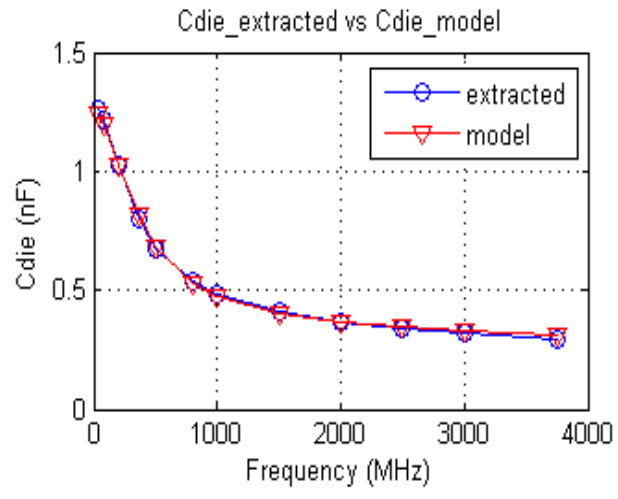

(b)

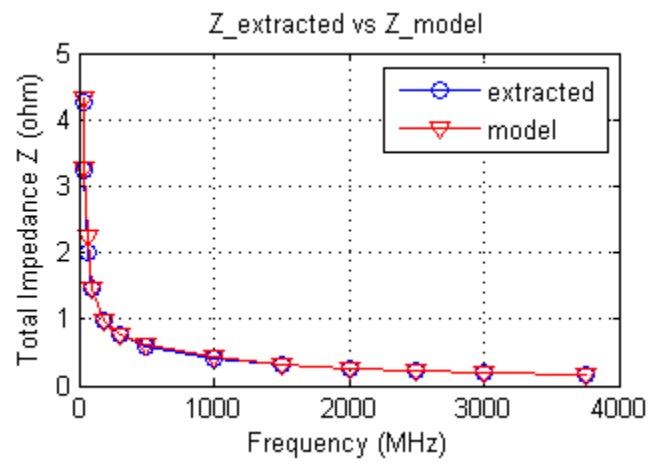

(c)

Figure 5.23: Comparison of the simulation results from extracted netlist and the approximate broadband equivalent circuit. (a) Rdie; (b) Cdie; (c) Impedance of series Rdie and Cdie. 


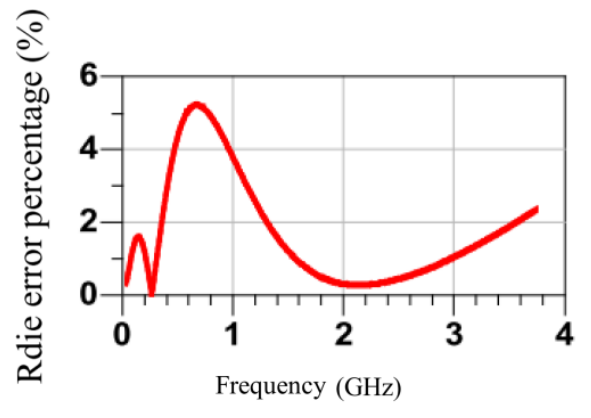

(a)

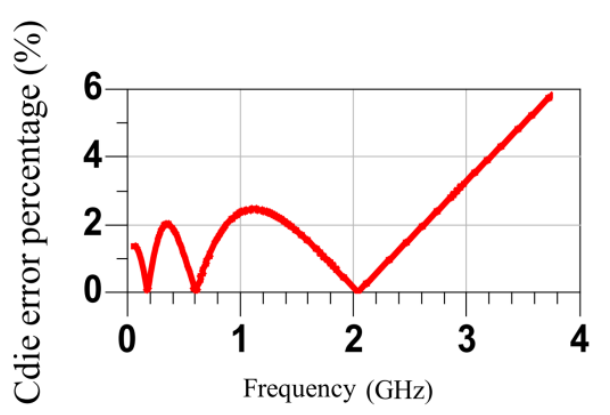

(b)

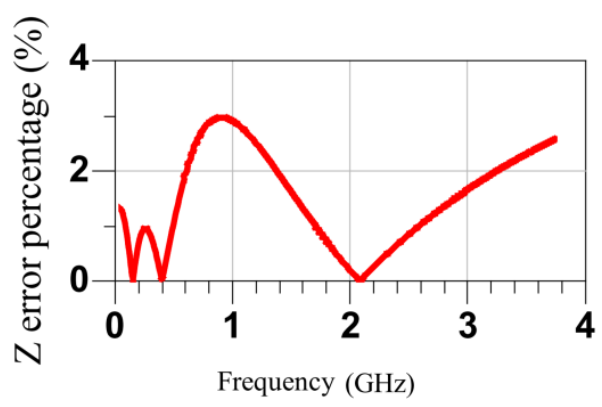

(c)

Figure 5.24: Rdie, Cdie and total impedance $Z$ error percentage of the approximate broadband equivalent circuit to the extracted results. (a) Rdie error percentage; (b) Cdie error percentage; (c) Total impedance error percentage.

\subsection{Summary}

Methods of extracting Rdie and Cdie using SPICE-like simulators have been introduced and discussed. A method of converting narrowband frequency domain Rdie and Cdie to a broadband equivalent circuit is proposed. This proposed method provides a means for SI engineers to perform time domain transient simulations to plot eye-diagrams for jitter estimation using available narrowband data without extra costs on commercial tools. 


\section{Chapter 6}

\section{Measurement of Rdie and Cdie}

\subsection{Introduction}

A low-cost method of pre-silicon Rdie/Cdie extraction by using a SPICE-like simulator has been proposed in Chapter 5. In this chapter, first the method of measuring low impedance at high frequency is covered and then a correlation measurement of Rdie/Cdie is performed.

\subsection{Measuring Impedance at the Milliohm Level}

Measurement of low impedance at high frequency can be especially challenging [77-80]. Two-port VNA connections greatly reduce the error due to the series connection impedance,

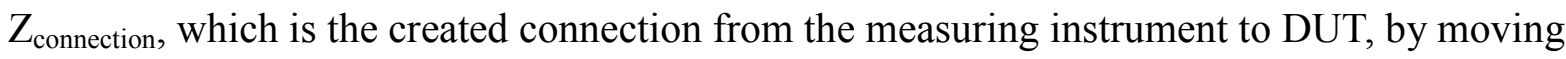
them into the 50-ohm VNA ports [71]. The method of using a VNA 2-port to measure low impedance is shown in Figure 6.1. Since $Z_{\text {connection } 1} \approx Z_{\text {connection2 }}<<Z_{\mathrm{SRC}}=Z_{R C V R}=50 \Omega$, the setup in Figure 6.1 can be approximated to that in Figure 6.2, and then the following equations can be obtained, 


$$
\begin{aligned}
& \mathrm{Z} 11=\mathrm{Z} 22=\mathrm{Z} 12=\mathrm{Z} 21=\mathrm{Zdut} \\
& \mathrm{S} 21=2 * \mathrm{Z} 12 * \mathrm{Z} 0 / \Delta \mathrm{Z} \\
& \Delta \mathrm{Z}=(\mathrm{Z} 11+\mathrm{Z} 0)(\mathrm{Z} 22+\mathrm{Z} 0)-\mathrm{Z} 12 \mathrm{Z} 21 \\
& \mathrm{Zdut}=25 * \mathrm{~S} 21 /(1-\mathrm{S} 21)
\end{aligned}
$$

where $\mathrm{Z} 0,50 \Omega$, is the characteristic impedance of the VNA port.

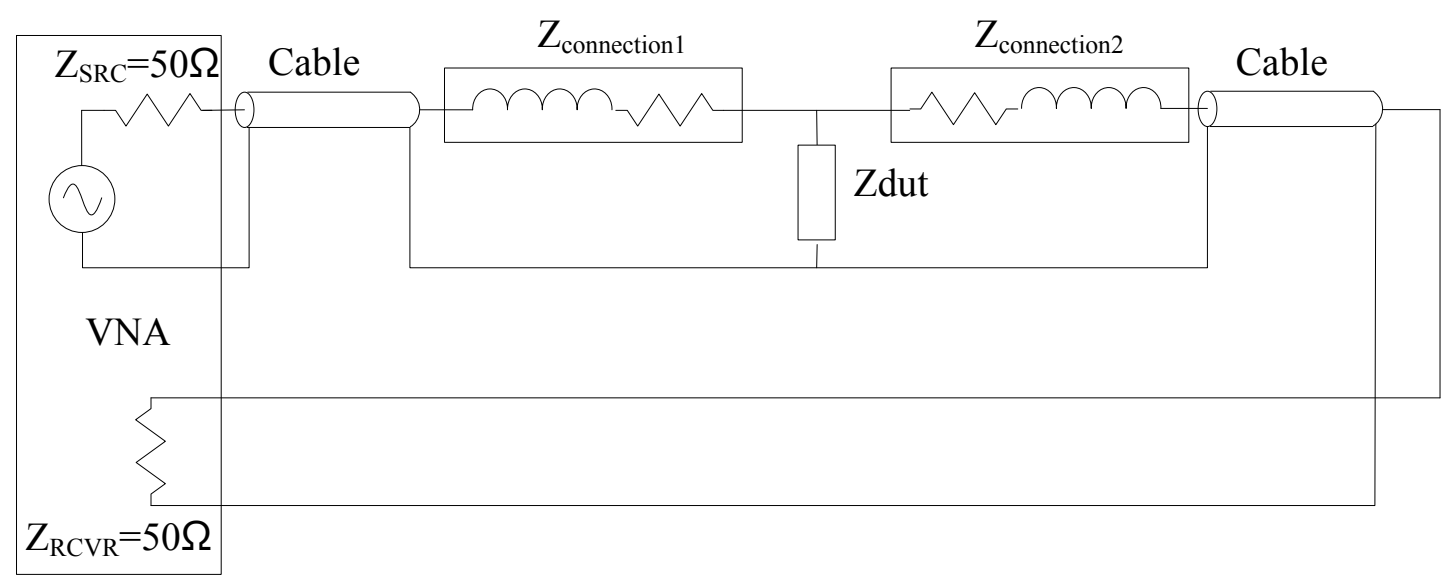

Figure 6.1: A method of using a VNA 2-port for measurement of low impedance 


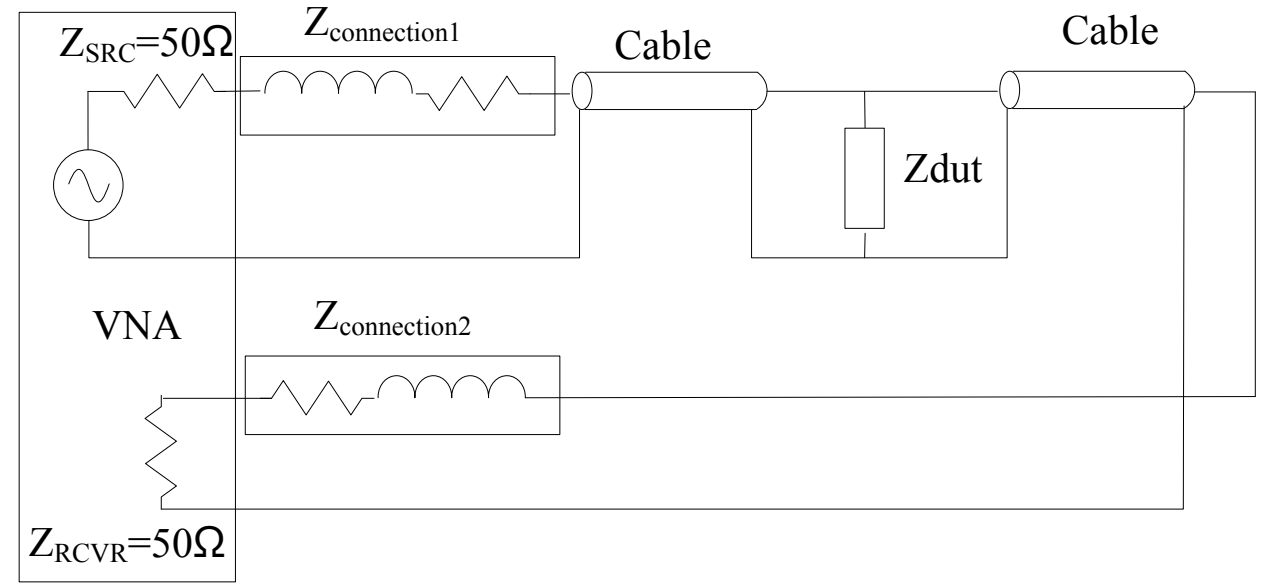

Figure $6.2: \mathrm{Z}_{\text {connection } 1}$ and $\mathrm{Z}_{\text {connection2 }}$ approximately absorbed by $\mathrm{Z}_{\mathrm{SRC}}$ and $\mathrm{Z}_{\mathrm{RCVR}}$ respectively.

Zdut is a complex number, the real part of Zdut is equivalent to the series resistance which is Rdie and the imaginary part of Zdut is contributed by the equivalent capacitance which is Cdie and inductance which is mainly from the package. At low frequency, the imaginary part of Zdut is dominated by Cdie and at high frequency the imaginary part of Zdut is dominated by the package inductance. So Rdie and Cdie can be expressed as,

$$
\begin{gathered}
\text { Rdie }=\operatorname{real}(\text { Zdut }) \\
\text { Cdie }=1 /(2 \pi * \text { freq } * \text { abs }(\text { imag }(\text { Zdut })))
\end{gathered}
$$

Because Cdie can be calculated by equation (6.3) and the resonant frequency can be obtained through the measurement, the package inductance value can be calculated through the following equation,

$$
f_{\text {resonant }}=1 /\left(2 \pi \sqrt{\left(C_{\text {die }} L_{p k g}\right)}\right)
$$


Here, it must be noticed that the Cdie obtained from a measurement (or equation (6.3)) is accurate only at low frequency. This is caused by instrument limitation.

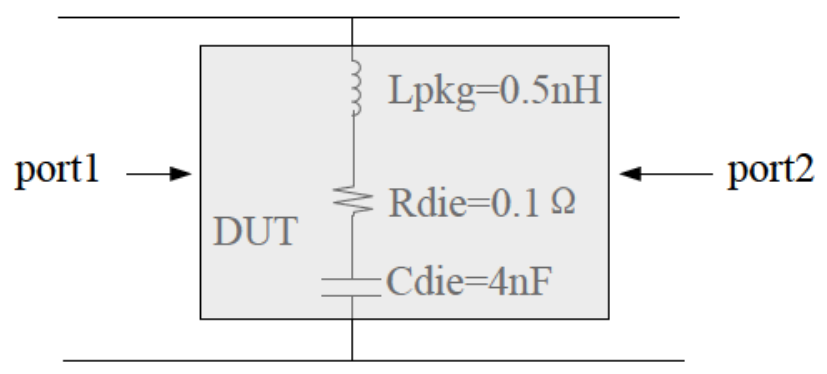

Figure 6.3: A RLC circuit.

A series RLC circuit in Figure 6.3 is used as an example to shown how the equations (6.1) to (6.4) are used to calculate Rdie and Cdie. In Figure 6.3, the ideal inductor, resistor and capacitor have been used and the values are listed in the figure.

The circuit is simulated by the simulator of Advanced Design System (ADS). The S21 of the RLC circuit can be directly read from the simulation results and the impedance Zdut of the RLC circuit can be calculated from equation (6.1). The imaginary part of the impedance Zdut of the RLC circuit is plotted in Figure 6.4. The imaginary part of the impedance curve exhibits a typical capacitive behavior ( $20 \mathrm{~dB}$ per decade drop-off) at low frequencies and a typical inductive ramp up at higher frequencies than the series resonant frequency. In between, the minimum point of the curve is the resonant frequency point. 


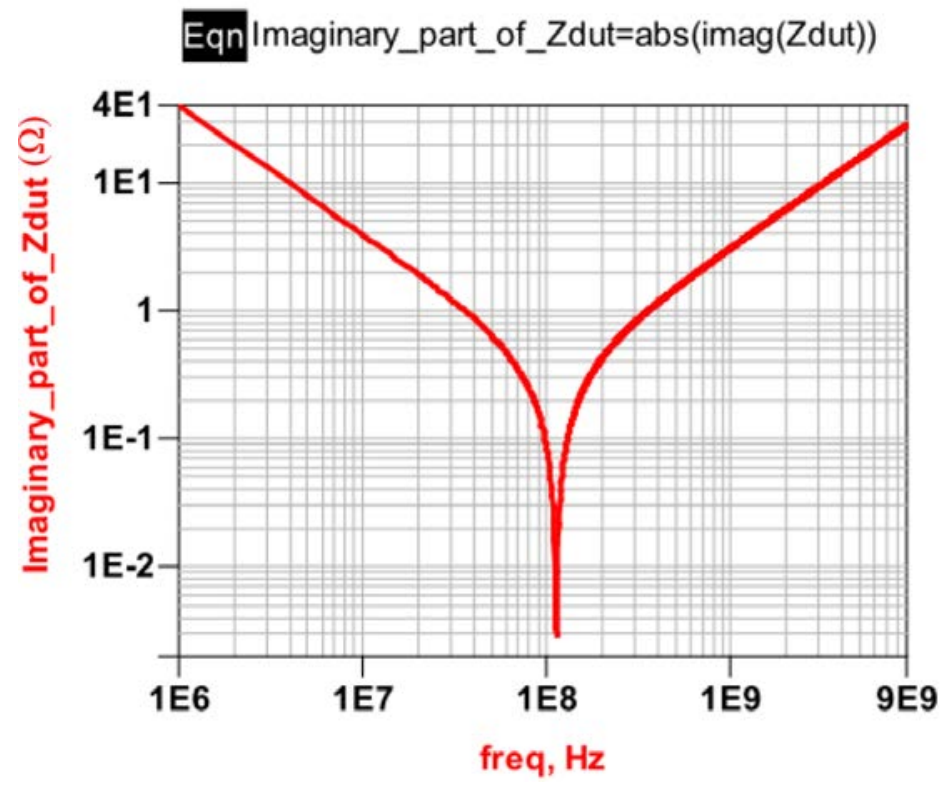

Figure 6.4: The imaginary part of the impedance Zdut measured from the circuit shown in Figure 6.3.

Form the low frequency range of the imaginary part of Zdut shown in Figure 6.4 and equation (6.3), the Cdie can be calculated. Since Zdut is obtained, from equation (6.2), the Rdie can be directly obtained.

\subsection{Rdie/Cdie Measurement of DDR Controller Test Chips}

Because the test chips are mounted on packaging substrate, it is impossible to measure bare die due to instrument limitations. Power (VDD) and ground (VSS) pads/BGA (ball grid array) balls at the packaging substrate are the access points for measurement. When one looks into VDD/VSS of the BGA pin with die attached, the equivalent circuit is shown in Figure 6.5. Test devices used in the measurement are listed in Table 6.1. 


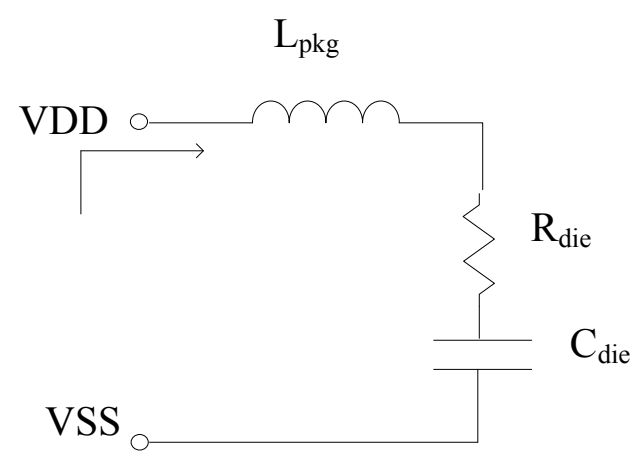

Figure 6.5: Equivalent circuit looking into VDD/VSS.

Table 6.1 Test devices for the measurement

\begin{tabular}{|c|}
\hline Test devices \\
\hline Agilent network Analyzer E5071C $(100 \mathrm{kHz}-8.5 \mathrm{GHz})$ \\
\hline HS probe station and RF/microwave probes (up to $12 \mathrm{GHz})$ \\
\hline TCS50 calibration substrate for HS probe \\
\hline Amscope optical microscope \\
\hline External Agilent DC power supply \\
\hline
\end{tabular}

The top level measurement setup is shown in Figure 6.6 and a detailed equipment arrangement is shown in Figure 6.7. The device under test (DUT) is placed face-down on a substrate probe station. The VNA ports connect to probe tips through coaxial cables. The probe tips are landed on the power/ground balls of the DUT. The VNA forces a signal on one pair of the power/ground pins and measures the DUT response on another pair of the power/ground pins. The E5071C VNA comes with an internal bias T to supply DC biasing 
through the probe tip while blocking DC signals going back to the receiver.

VNA calibration was performed with TCS50 calibration substrate (shown in Figure 6.8) provided by the probe vendor. The two port full calibration SOLT (short, open, load and through) [81] is used.

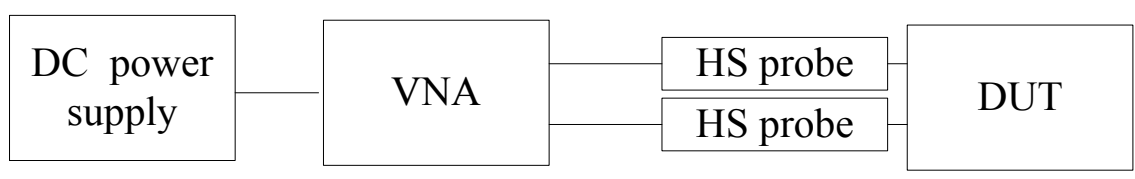

Figure 6.6: Top level measurement setup.

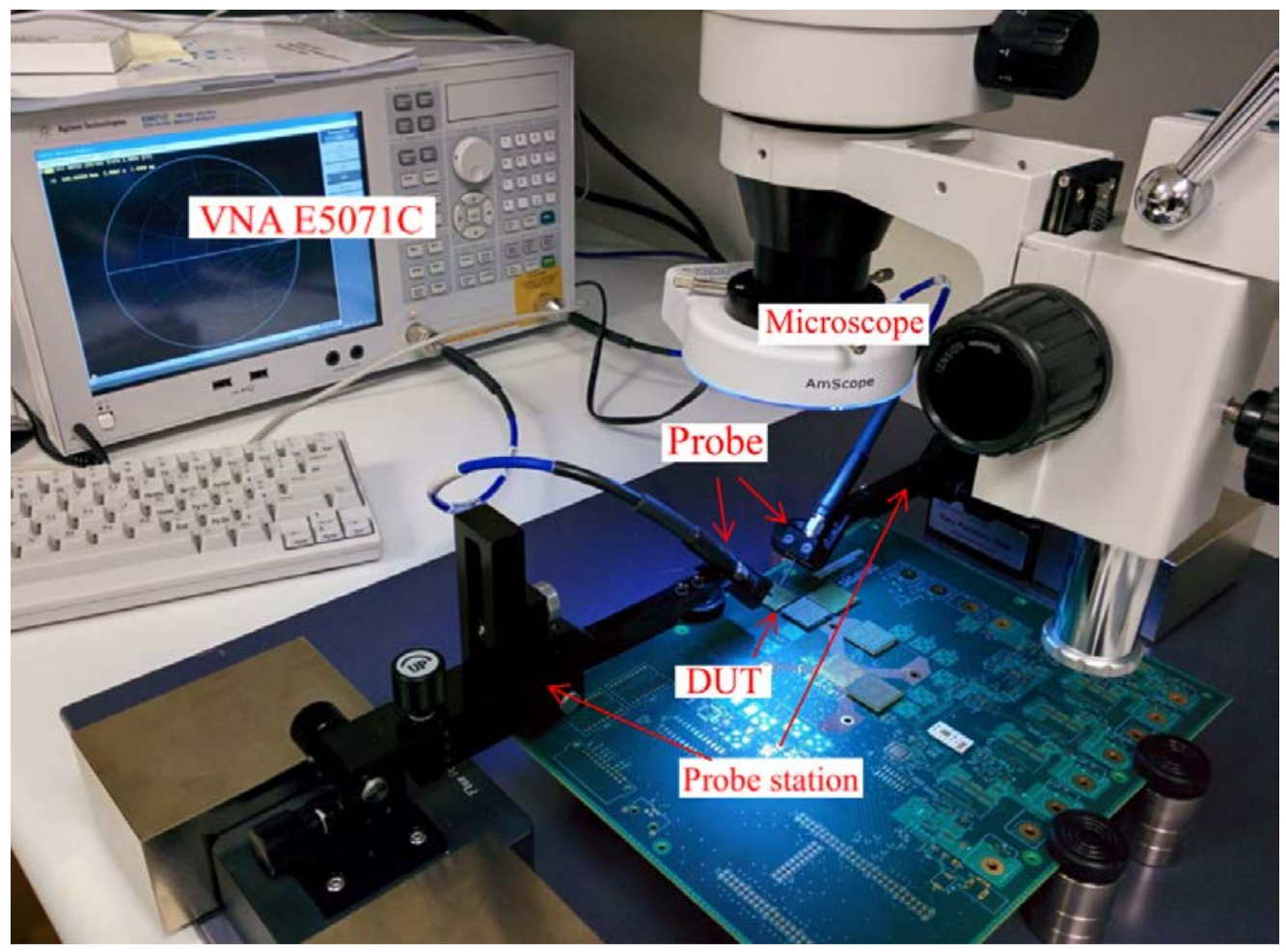

Figure 6.7: The detailed equipment arrangement for the measurement setup. 


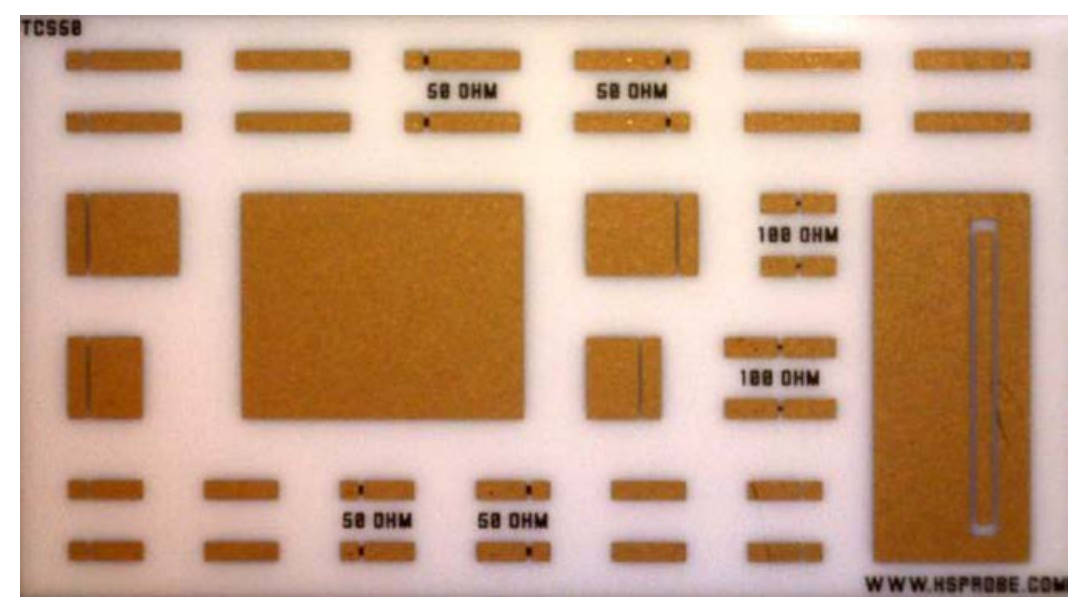

Figure 6.8: TCS50 calibration substrate.
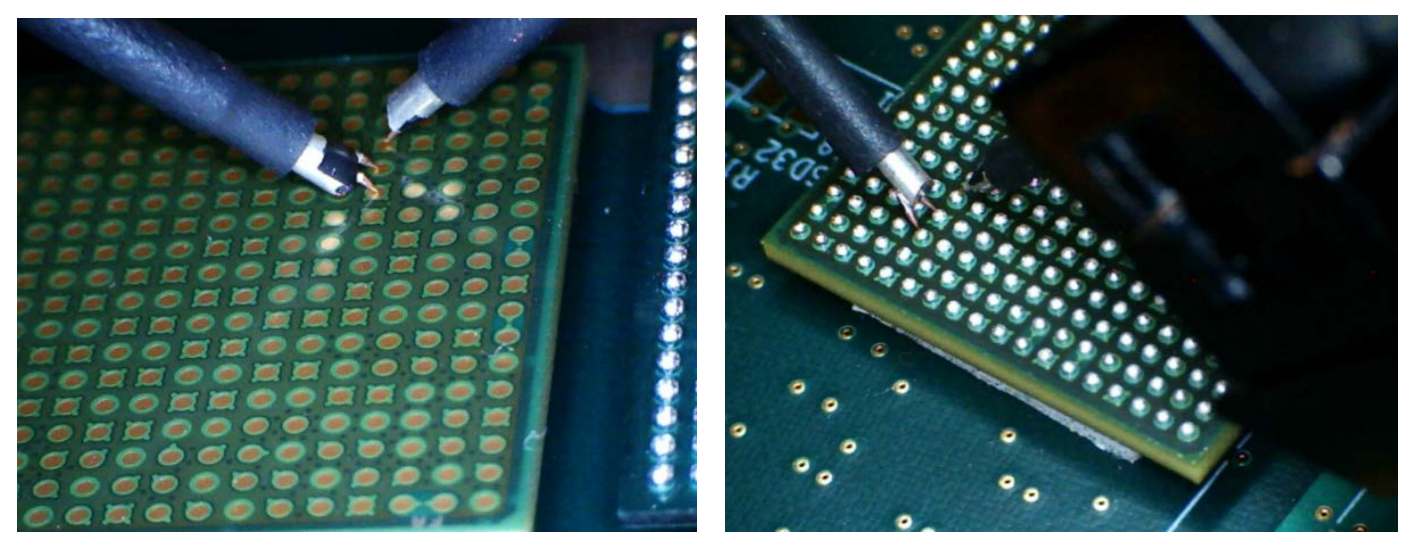

Figure 6.9: Probes on the bare substrate and packaged devices.

Figure 6.9 shows probes on the bare substrate and packaged devices. Two pairs of VDD/VSS pins side by side were chosen as measurement points. They need to be close to avoid spatial error, so that mutual inductive coupling between probes is minimized [70]. 


\subsubsection{Extracting Decoupling Capacitance Cpkg and Inductance Lpkg on a Bare Package Substrate}

The impedance Zdut of bare substrate for both flip chip bonding [82] and wire bonding [83] chip has been measured. The magnitudes of imaginary parts of the impedance Zdut have been plotted in Figure 6.10. The curve for wire bond packing is marked with a red diamond and the curve for the flip chip is marked with a blue triangle. It can be seen that the bare substrate resonant frequency for both types are around $1 \mathrm{GHz}$. This is because the series Lpkg and the Cpkg for the bare substrate are small and the architectures for both are similar. Below the $1 \mathrm{GHz}$ resonant frequency, both curves have a $20 \mathrm{~dB}$ per decade drop-off, which is a typical characteristic of capacitance.

According to (6.1) and (6.4), the impedance Zdut of the bare substrate is calculated; the substrate inductance, Lpkg, and substrate capacitance, Cpkg, are obtained. The calculation results are listed in Table 6.2. The measured ESRs of substrates are plotted in Figure 6.11. The ESR of the bare substrate for the flip chip is marked with blue diamonds and the one for the wire bonding chip is marked with red squares. 
Table 6.2 Measured Cpkg and Lpkg of the flip chip substrate and the wire bonding chip substrate

\begin{tabular}{|c|c|c|}
\hline & Flip chip & Wire bond \\
\hline Cpkg $(\mathrm{pF})$ & 640 & 140 \\
\hline $\operatorname{Lpkg}(\mathrm{pH})$ & 35 & 200 \\
\hline
\end{tabular}

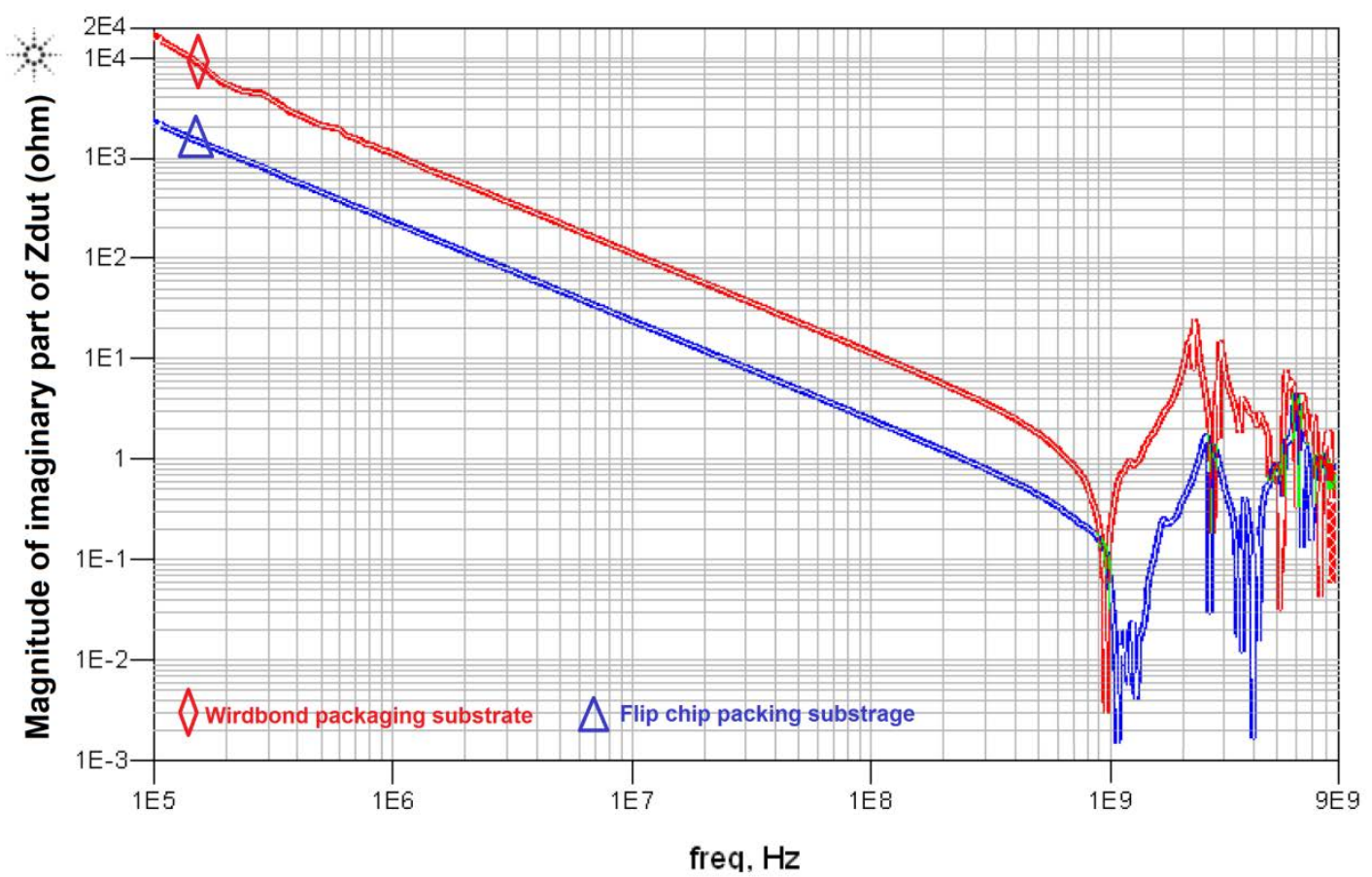

Figure 6.10: The imaginary parts of the bear substrates of the flip chip and the wire bond chip. 


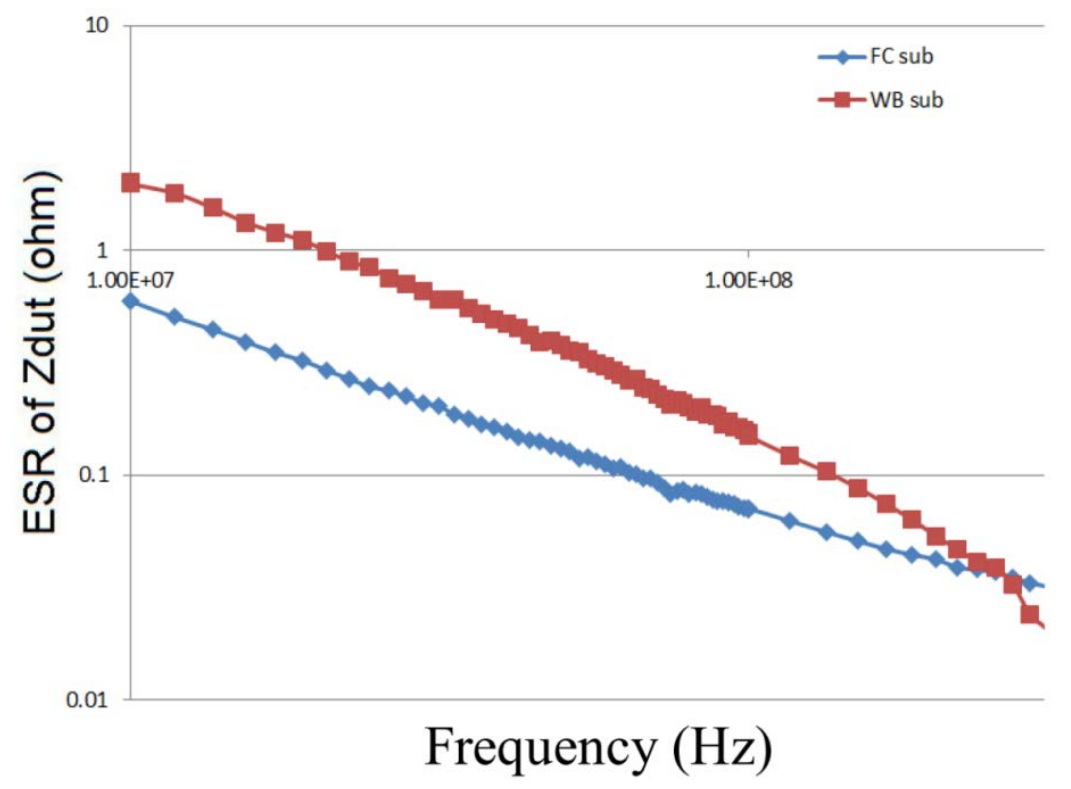

Figure 6.11: Extracted ESR of substrates.

\subsubsection{Extracting Cdie and Lpkg on Packaged Devices}

The PDNs on wire bond packaging [83] and flip chip packaging [82] devices are shown in Figure 6.12 (a) and (b) respectively. Comparing these two architectures, one can easily find that the wire bond packing devices need extra bond wire to connect the PDN on die and the PDN on substrate. The wire bond introduces relatively large inductance. For a flip chip, the on-die PDN and on-substrate PDN are connected through bumps directly. The inductance introduced by bumps can be ignored.

In this test, three test chips which are chipA, chipB and chipC have been measured. The 
magnitudes of the imaginary parts of Zdut have been plotted in Figure 6.12. The plot for chipA is marked with a pink circle, for chipB is marked with a blue triangle and for chipC is marked with a red diamond. ChipA is wire bonding, chipB and chipC are flip chip bonding. It can be seen that all of the resonant frequencies are between $100 \mathrm{MHz}$ and $300 \mathrm{MHz}$. These frequencies are lower than that of the bare substrate, which is around $1 \mathrm{GHz}$. This is because the series Lpkg and Cdie for packaged devices are relatively large. To calculate Cdie by using equation (6.3), one must find the capacitance dominant frequency range which has the characteristic of a $20 \mathrm{~dB}$ per decade drop-off for the magnitudes of imaginary part of Zdut shown in Figure 6.13. For chipB, it can be easily and accurately calculated between $500 \mathrm{KHz}$ and $50 \mathrm{MHz}$. For chipA, it can be calculated during the frequency range between $5 \mathrm{MHz}$ and $30 \mathrm{MHz}$. For chipC, it is difficult to find a wide frequency range with $20 \mathrm{~dB}$ per decade drop-off, so the measured Cdie value may not be highly accurate.

According to (6.1) and (6.4), the impedance $Z_{\mathrm{DUT}}$ for the three test chips has been calculated and the extracted Lpkg and Cdie are calculated and the results are listed in Table 3. The extracted ESRs are plotted in Figure 6.14. 


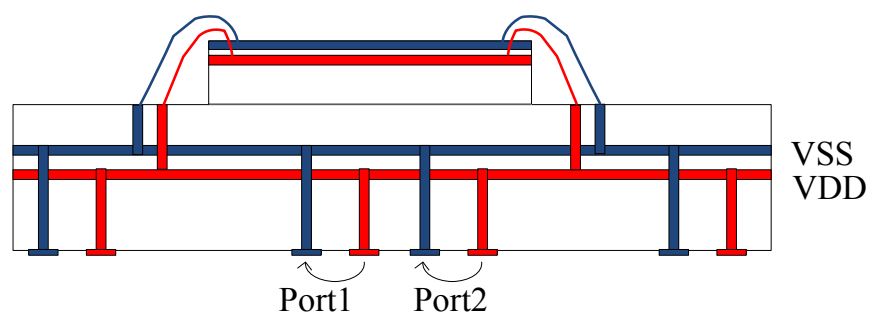

(a)

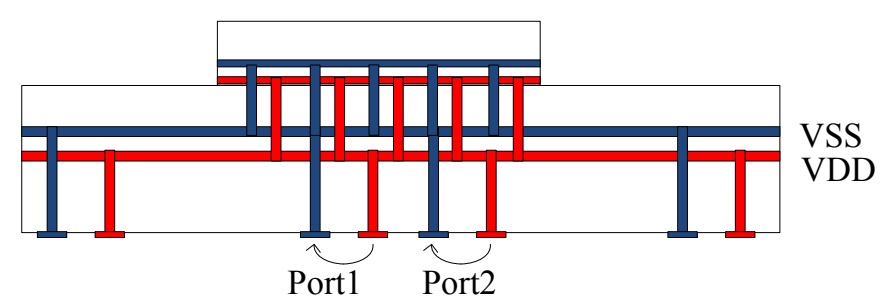

(b)

Figure 6.12: Packaged devices PDN for (a) the wire bond and (b) the flip chip packing. 


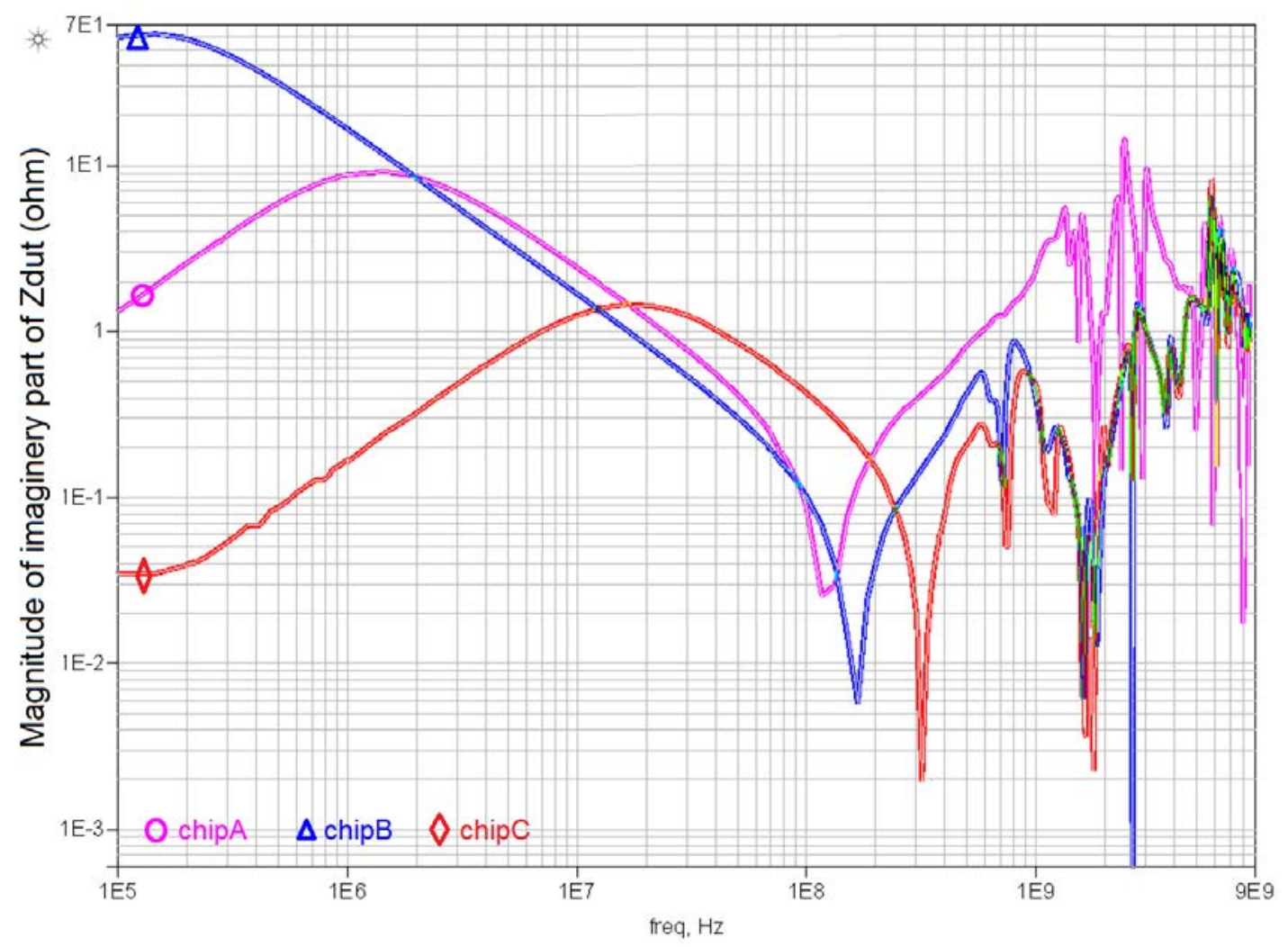

Figure 6.13: The measured imaginary parts of Zdut of devices with the flip chip or the wire bond package. 


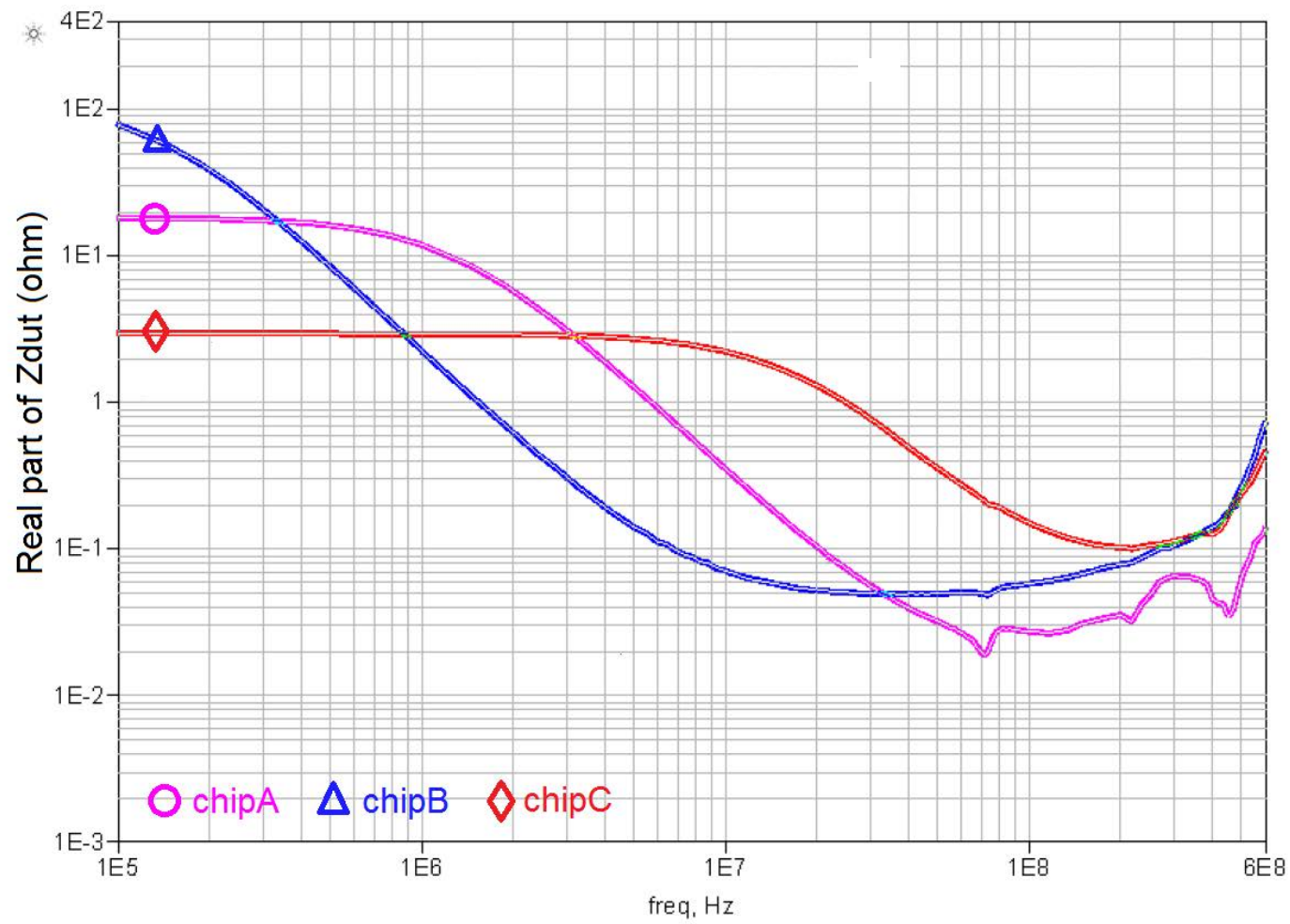

Figure 6.14: Extracted ESR of packaged devices.

Table 6.3 Extracted Cdie and Lpkg of packaged devices

\begin{tabular}{|c|c|c|c|}
\hline & chipA (wire bond) & chipB (flip chip) & chipC (flip chip) \\
\hline Cdie $(\mathrm{nF})$ & 6.6 & 9.2 & 3.7 \\
\hline $\operatorname{Lpkg}(\mathrm{pH})$ & 280 & 96 & 68 \\
\hline
\end{tabular}

In Table 6.3, it can be seen that package inductance of the wire bonding chip is larger than that of the flip chip. And from the measurement setup one can see that the measured Lpkg includes both Lpkg of the substrate and the inductance after package substrate to Cdie because one can only access PDN from the BGA (ball grid array) pads. 


\subsubsection{Improved Extracted Series RLC Model vs. Original Measured S21}

To verify whether the RLC model with measured parameters of Lpkg, Rdie and Cdie is accurate, the S21 of RLC model and the original measured S21 of chipA has been plotted and shown in Figure 6.16. The $\mathrm{S} 21$ of RLC model is $\mathrm{dB}(\mathrm{S}(2,1))$ and the original measured S21 of chipA is dB(chipA..S(2,1)). Figure 6.15 shows the measured Rdie, Cdie and Lpkg. Rdie equals $0.03 \Omega$, which is the value at the resonant frequency read from Figure 6.14. Cdie is $6.6 \mathrm{nF}$ and $\mathrm{Lpkg}$ is $280 \mathrm{pH}$ when one reads from Table 6.3. From Figure 6.16 one can see that the extracted model S21 matches the original measured S21 in a relatively wide range $(5 \mathrm{MHz}$ to $700 \mathrm{MHz})$ around the resonant frequency.

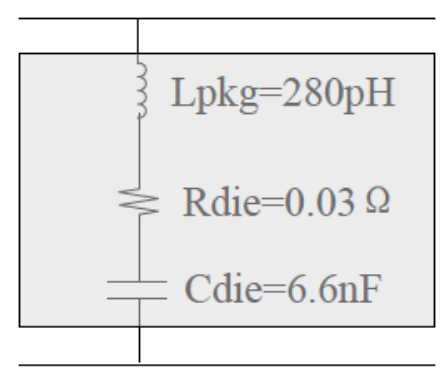

Figure 6.15: Extracted series RLC model of chipA. 


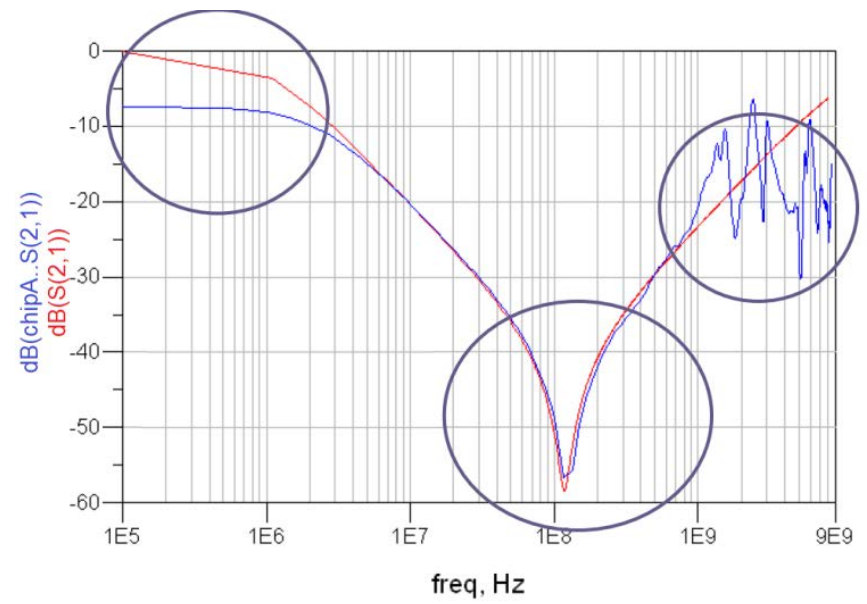

Figure 6.16: Original measured S21 of chipA vs. extracted RLC model S21.

One should notice that Rdie and Cdie are dynamic values. They are highly dependent on the circuit operation state, power supply voltage temperature and process variation. The accuracy of the measured Cdie is also limited in a narrow frequency range.

The extracted model S21 matches the original measured S21 in a relatively wide range but the low frequency response and the secondary resonance are not captured correctly. To improve the low frequency response, a shunt resistor in parallel with Rdie is added. The value of the resistor is $18 \Omega$, which is the Rdie at the lowest frequency as shown in Figure 6.17.

It has been mentioned earlier that the extracted Lpkg includes two parts. One can separate these two parts into Lpkg_sub and Lpkg_die. Lpkg_sub is the inductance on the bear substrate, and Lpkg_die is the total extracted inductance Lpkg subtracting Lpkg_sub.

In the case of chipA, the package inductance, Lpkg_sub $=200 \mathrm{pH}$, 
Lpkg_die $=280 \mathrm{pH}-200 \mathrm{pH}=80 \mathrm{pH}$, Rleak $=18 \Omega$, the ESR on substrate Rsub $=0.1 \Omega$ and ESR on die $\mathrm{Rdie}=0.03 \Omega, \mathrm{Cpkg}=140 \mathrm{p}$ and $\mathrm{Cdie}=6.6 \mathrm{nF}$. A relatively more accurate $\mathrm{RLC}$ model is created and shown in Figure 6.17.

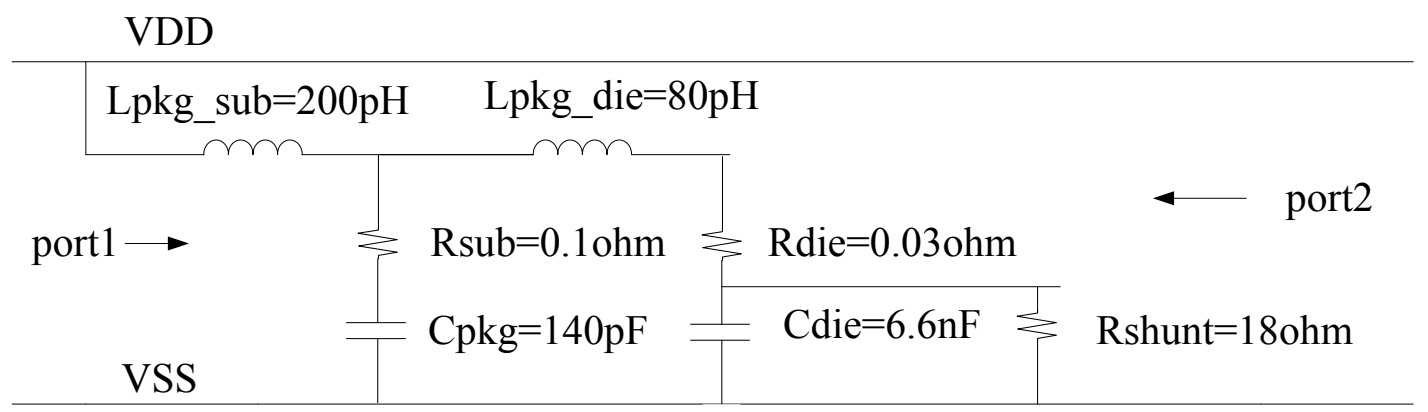

Figure 6.17: Improved extracted RLC model of chipA.

The S21 of the improved extracted equivalent model and the original measured S21 of chipA have been plotted and shown in Figure 6.18 It can be seen that the S21 of the improved extracted model matches the original S21 from low frequency to secondary resonance frequency $(100 \mathrm{KHz}$ to $1 \mathrm{GHz})$. 


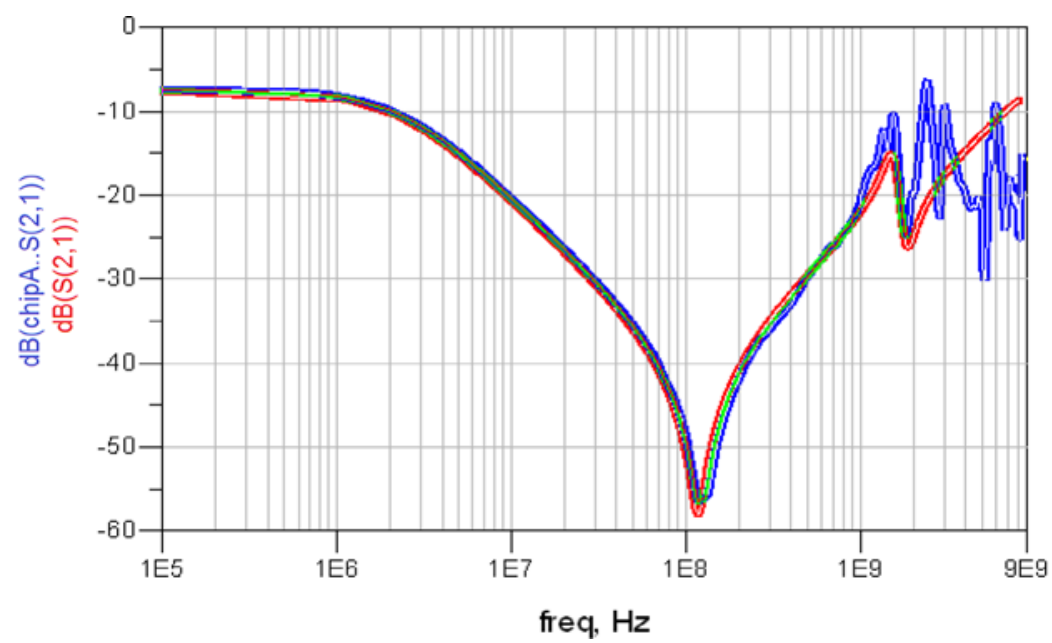

Figure 6.18: Original measured S21 of chipA vs improved extracted RLC model S21.

With the same method applied to chipB and chipC, the results are shown in Figure 6.19, Figure 6.20, Figure 6.21 and Figure 6.22 respectively. For chipB, the quiescent current is very small that it can be ignored. As mentioned in Section 6.3.2, that the Cdie value of chipC may not be accurately measured because it is difficult to find a wide frequency range with $20 \mathrm{~dB}$ per decade drop-off for the magnitude of the imaginary part of the Zdut shown in Figure 6.15. From Figure 6.22 one can see that the S21 of the extracted RLC does not match the original measured S21 of chipC in the frequency range between $10 \mathrm{MHz}$ and $500 \mathrm{MHz}$. This is caused by the inaccurate Cdie measurement. 


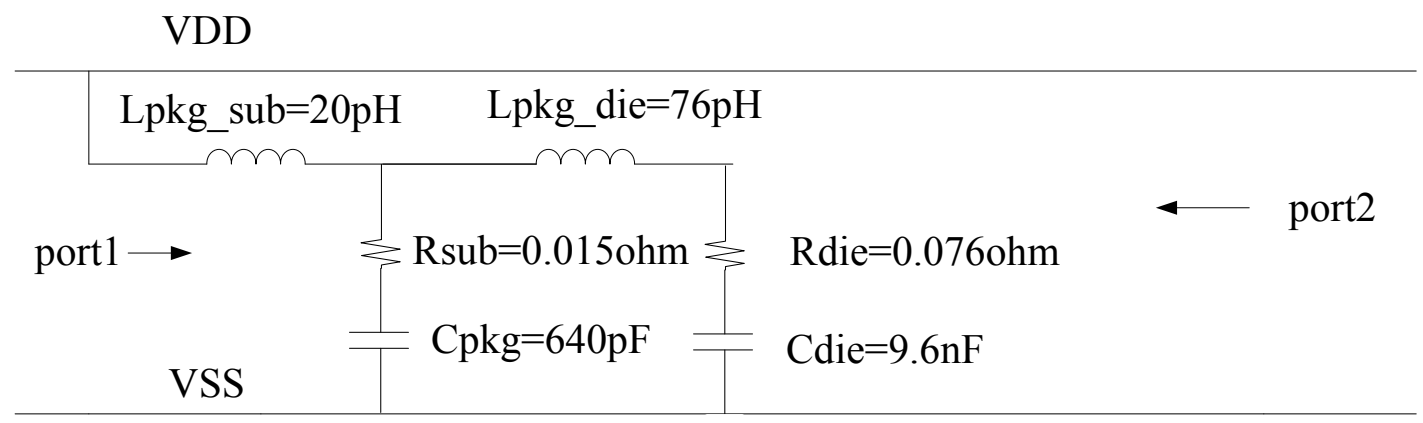

Figure 6.19: Improved extracted series RLC model of chipB.

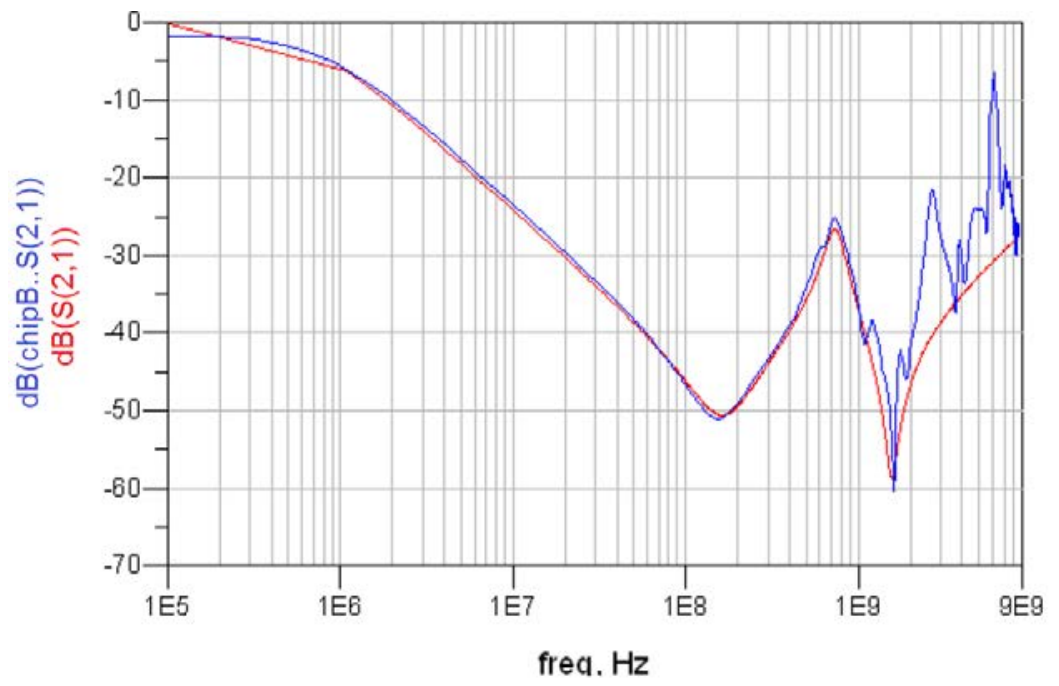

Figure 6.20: Original measured S21 of chipB vs. improved extracted RLC model S21. 


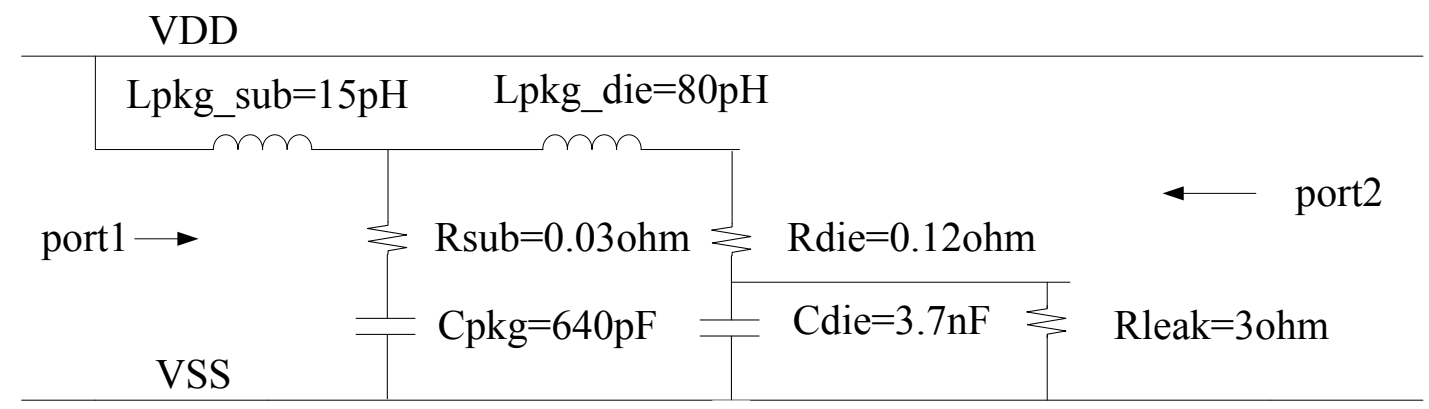

Figure 6.21: Improved extracted series RLC model of chipC

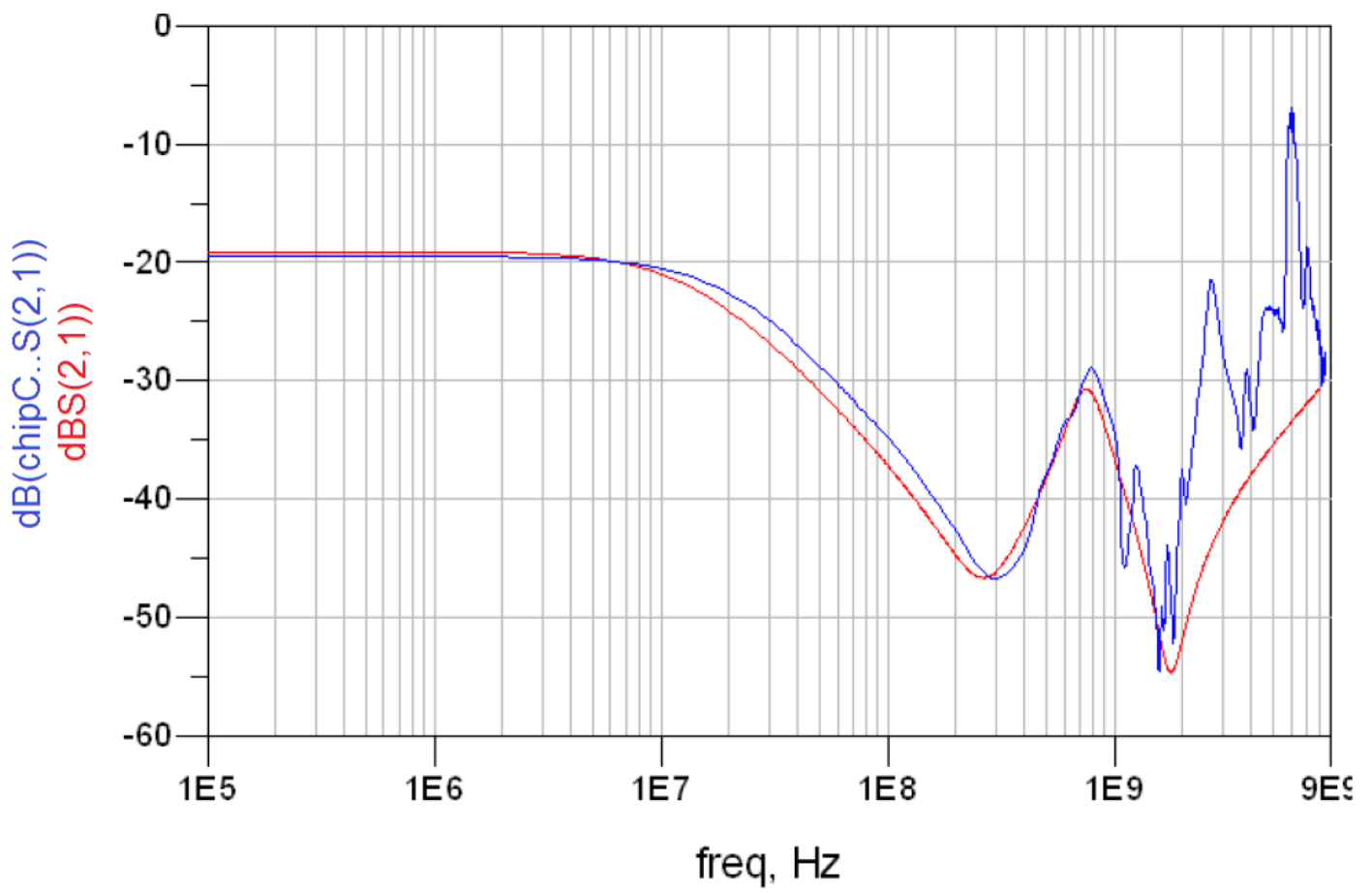

Figure 6.22: Original measured S21 of chipC vs. improved extracted RLC model S21.

In order to compare the accuracy of the improved RLC model with the original test chip, the error percentage is defined as follows, 


$$
\text { Error_percentage }=\left|\frac{Z_{\text {model }}-Z_{\text {chip }}}{Z_{\text {chip }}}\right| \times 100 \%
$$

where $Z_{\text {model }}$ is the total impedance of the improved RLC model for the three test chips as shown in Figure 6.17, Figure 6.19 and Figure 6.21 respectively, $Z_{\text {chip }}$ is the measured test chip impedance from equation (6.1).

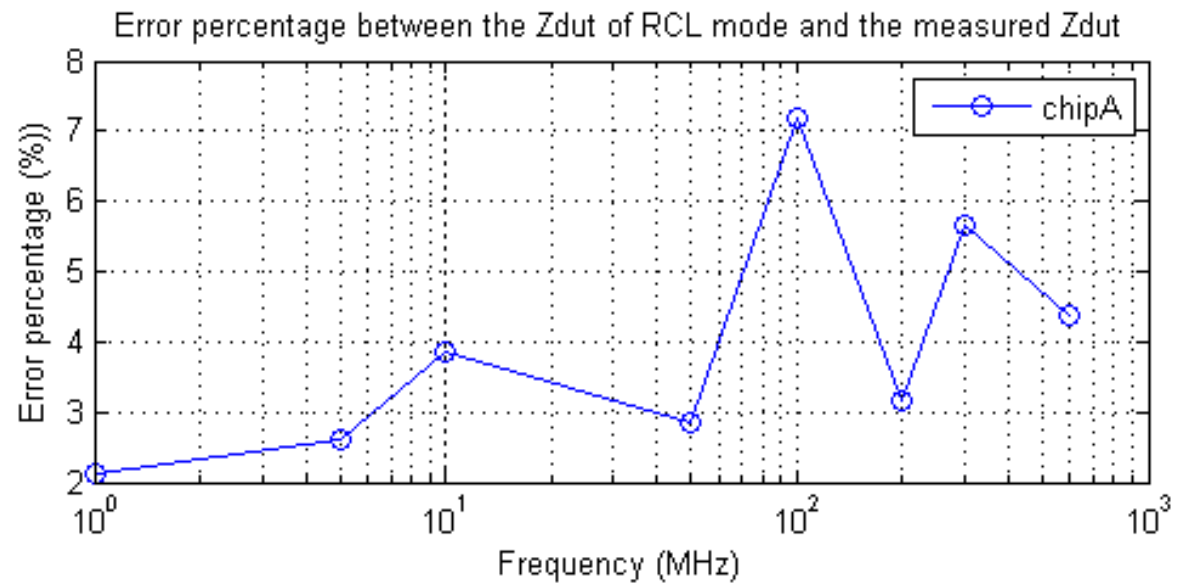

Figure 6.23: Error percentage for chipA.

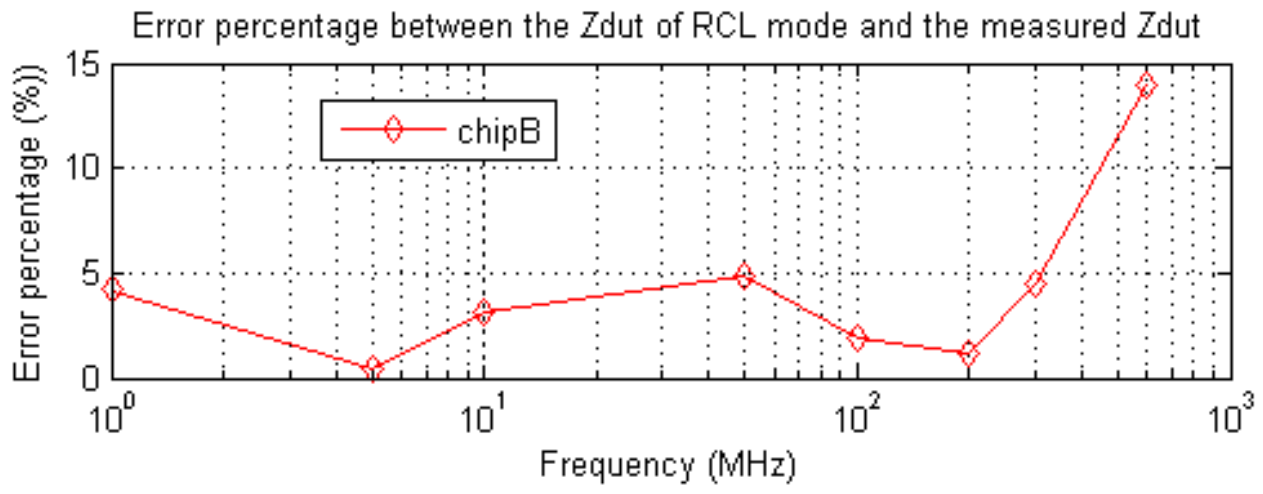

Figure 6.24: Error percentage for chipB. 


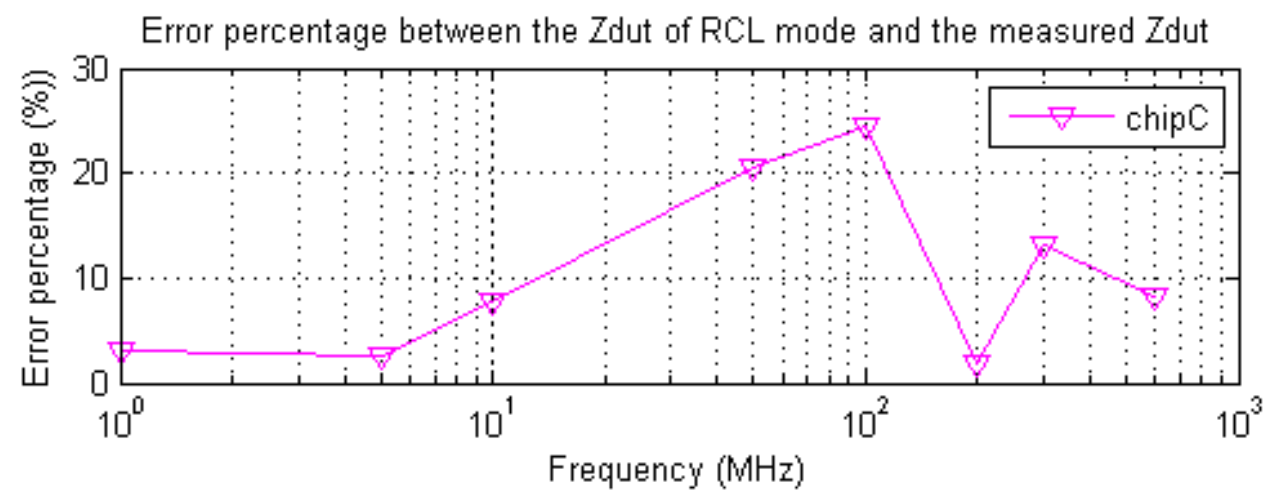

Figure 6.25: Error percentage for chipC.

The calculated error percentage for chipA is plotted in Figure 6.23. The largest error value occurs at $100 \mathrm{MHz}$ which is close to the resonant frequency (shown in Figure 6.18). During that frequency range the impedance is very close to Rdie which is about $30 \mathrm{~m} \Omega$ in this case. $7 \%$ error means a $2.1 \mathrm{~m} \Omega$ difference from the $30 \mathrm{~m} \Omega$ Rdie. The difference can be ignored due to its small value.

Figure 6.24 shows the error percentage plot for chipB. The maximum error happens at $600 \mathrm{MHz}$. In Figure 6.13 one can see that there is a small resonance which happens in that frequency range. Because the improved model only captures the main resonance and the first secondary resonance, other resonances cannot be captured. This limitation is caused by the measurement equipment and method. An on-die measurement can absolutely improve the accuracy, but the cost and feasibility of the measurement needs further exploration.

The error percentage plot for chipC is plotted in Figure 6.25. One can see that the error 
is around $20 \%$ to $25 \%$ in the frequency range between $50 \mathrm{MHz}$ and $100 \mathrm{MHz}$. The measurement method is based on the assumption that the imaginary part of the impedance Zdut curve exhibits a typical $20 \mathrm{~dB}$ per decade drop-off at low frequencies and Cdie can be accurately calculated by using the curve. But it is difficult to find a wide frequency range with $20 \mathrm{~dB}$ per decade drop-off for chipC. Consequently, the estimated Cdie value is not very accurate. This means that if the $25 \%$ error cannot satisfy the design requirement, other methods need to be found to improve the measurement accuracy for the case of ChipC.

\subsubsection{Comparison between Simulated Results and Measured Results}

The chipC consists of four subblocks. The four subblocks have exactly the same circuits which are the Circuit-A but different signal definitions. The Rdie/Cdie of the Circuit-A has been extracted with the extraction method covered in chapter 5 and the approximate broadband equivalent circuit is shown in Figure 5.23.

Because the four subblocks have the same circuits, one can assume that they have the same Rdie/Cdie. So the Rdie of chipC should be equal to four Rdie of each subblock in parallel and Cdie of chipC should be equal to four Cdie of each subblock in parallel. Four copies of the equivalent circuits in Figure 5.23 are placed in parallel to replace the Rdie/Cdie value in Figure 6.21. The new equivalent RLC model is shown in Figure 6.26. The S21 of the new RLC model shown in Figure 6.26 and the original measured S21 of chipC have been 
plotted and shown in Figure 6.27. It can be seen that the S21 of the new model matches the original S21 more accurately than the single Rdie/Cdie model shown in Figure 6.21. The deviation of the resonant frequency from the measurement result might be caused by the measurement temperature and process variation [73].

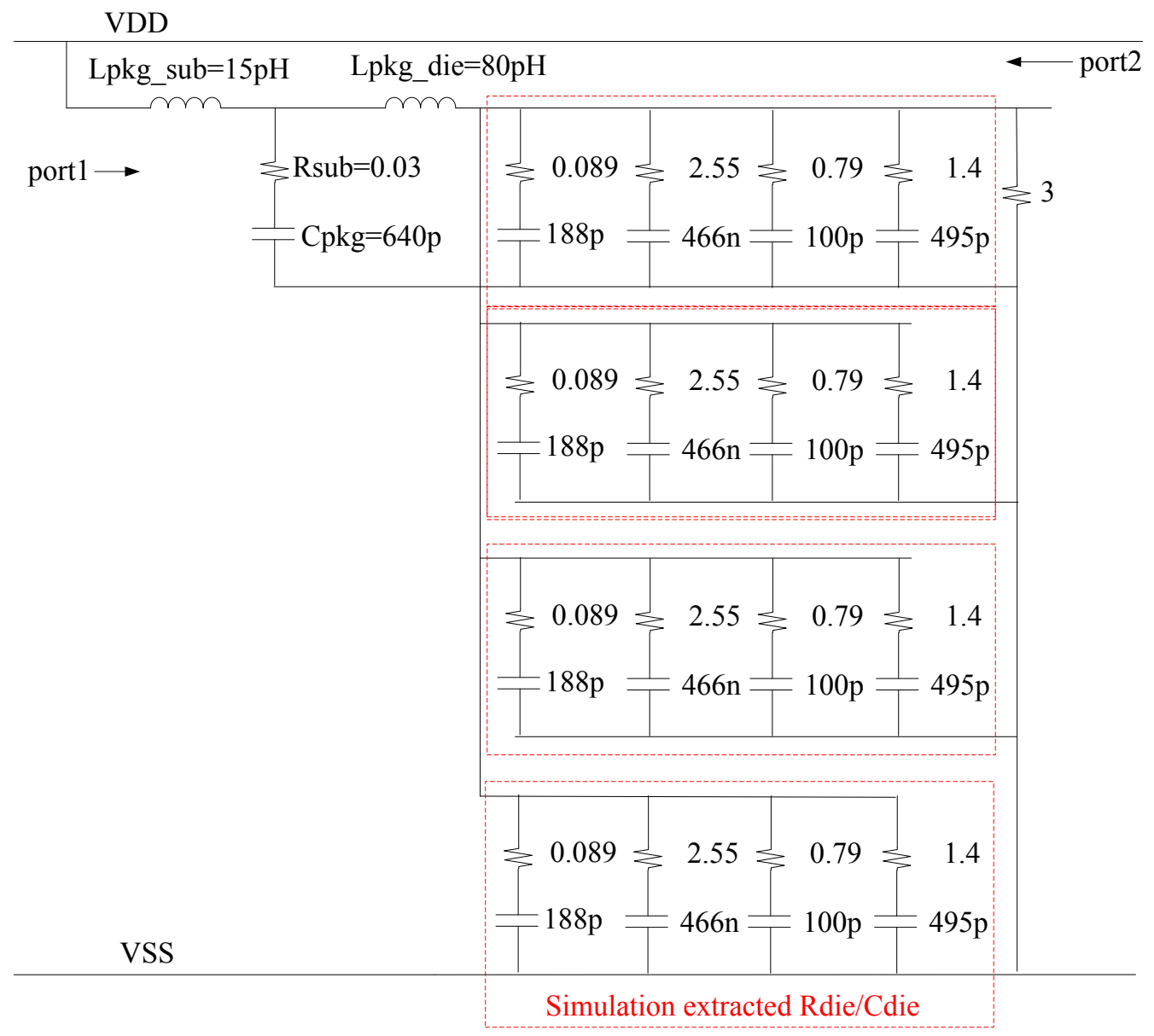

Figure 6.26: New extracted RLC model of chipC 


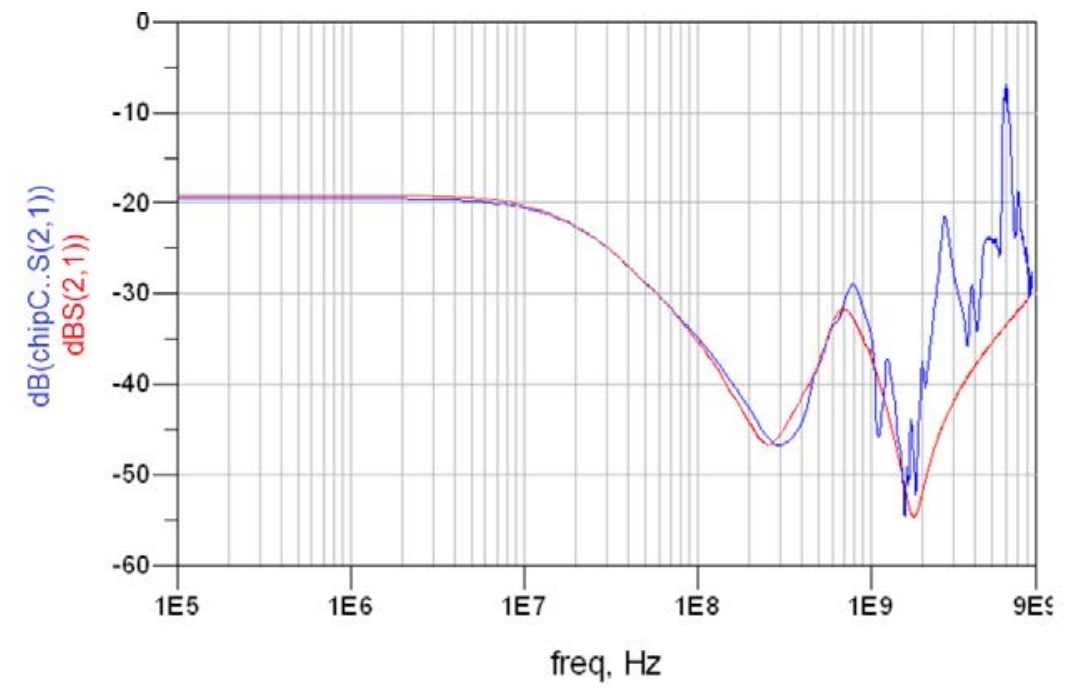

Figure 6.27: Original measured S21 of chipC vs. new extracted RLC model S21.

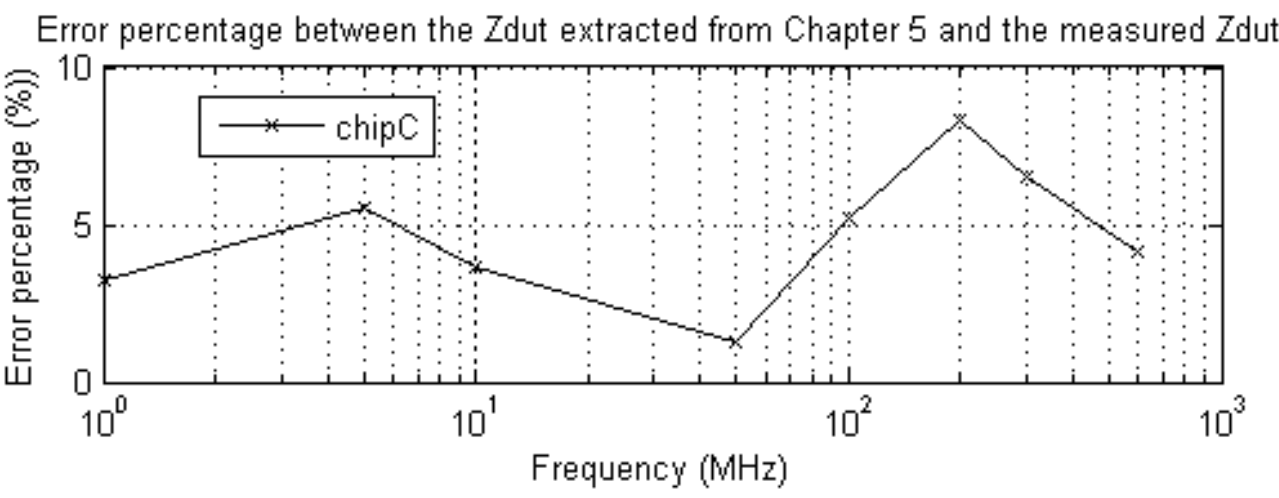

Figure 6.28: Error percentage for chipC.

Figure 6.28 shows the error percentage plot for the new RLC extracted model of chipC as shown in Figure 6.26 . The largest error happens during the $200 \mathrm{MHz}$ and $300 \mathrm{MHz}$ range which is in the resonance frequency range. Similar to the case of chipA, the $8 \%$ error is caused by about $10 \mathrm{~m} \Omega$ which is very small and can be ignored in most cases. 


\subsection{Summary}

In this chapter, the method of measuring low impedance at high frequency and the Rdie/Cdie measurement of DDR controller test chips are presented. The measured results have verified the effectiveness of the method of extracting Rdie/Cdie proposed in chapter 5. From the improved RLC models and their S21 plots as shown in Figure 6.15, 6.16, 6.17 and 6.18, one can see that the main resonance is dominated by Lpkg_sub+Lpkb_die and Cdiep; the secondary resonance is generated by $\mathrm{Lpkg} \_$sub and $\mathrm{Cpkg}$. One interesting observation is that at low frequencies, the PDN impedance may be affected by the quiescent current. 


\section{Chapter 7}

\section{A Case Study and Conclusion}

\subsection{A Design Case Study}

In order to demonstrate and compare how the traditional PLL based clock distribution circuit and the proposed MDLL based clock distribution circuit can affect the performance of the clock distribution circuits of a DDR controller, two simplified clock distribution circuits have been created. The circuits are shown in Figure 7.1.

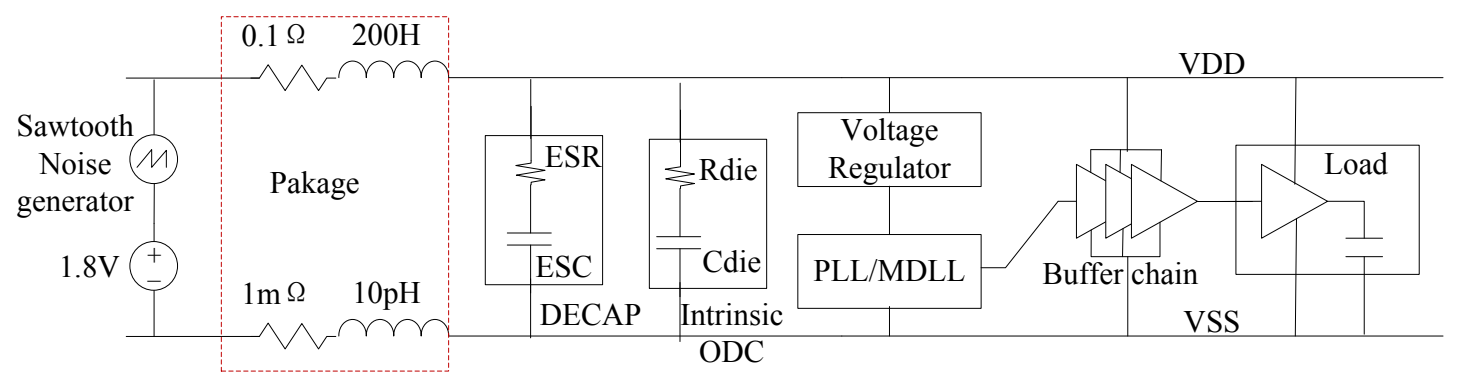

Figure 7.1: Simplified clock distribution circuits for a DDR controller based on a PLL or an MDLL.

The clock distribution circuits have either a PLL or an MDLL, a voltage regulator with a PSRR larger than $26 \mathrm{~dB}$ over $2 \mathrm{GHz}$, a long delay clock buffer chain, the Intrinsic ODC, and DECAP cell. The Intrinsic ODC is the original on-die decoupling capacitor. In order to simplify the test bench and reduce simulation time, it is assumed that the frequency domain Rdie and Cdie are the same as those shown in Table 5.1, which means that the on-die circuits 
in Figure 7.1 are similar to that of Circuit-A. This assumption is reasonable because there is a PLL on Circuit-A as well; in addition, the PLL/MDLL and the Buffer chain are much smaller circuits compared with Circuit-A. The DECAP is added only when the power supply noise amplitude is larger than the design specification. The time constant of ESR and ESC of the DECAP satisfies the condition of $\mathrm{ESR} * \mathrm{ESC}=1 \Omega * 360 \mathrm{pF}$. The PLL and MDLL are the same as the PLL and the MDLL designed in Chapter 3. The clock buffer chain is Buffer chain 4 in Table 4.1. The package inductance and resistance for the supply net are $200 \mathrm{pH}$ and $0.1 \Omega$, for the ground net are $10 \mathrm{pH}$ and $1 \mathrm{~m} \Omega$. The noisy supply voltage is modeled as a DC supply voltage whose value is $1.8 \mathrm{~V}$ and a sawtooth-shaped signal generator. The supply noise is modeled as a sawtooth-shaped signal which has the peak-to-peak amplitude of $200 \mathrm{mV}$ and $500 \mathrm{MHz}$ frequency. The noise is assumed to be generated from other on-die circuits whose power supply is connected with that of the clock distribution circuit on the BGA ball.

The design procedure to limit power supply induced jitter is presented in the following sections. Traditional design methods are used for the design of PLL based clock distribution circuit and the techniques proposed in this dissertation are used for the design of the MDLL based clock distribution circuit. 


\subsubsection{Sizing the DECAP to Satisfy the Supply Noise Specification}

It is one of the most important steps in system SI design to add DECAP to lower the supply noise. The on-die PDN must satisfy the supply noise specification which typically is less than $\pm 5 \%$ nominal supply voltage. The testbench of sizing the DECAP to satisfy the supply noise specification is shown in Figure 7.2. The $I_{\text {on-die }}$ is the current file of on-die circuits and is generated with the nominal supply voltage.

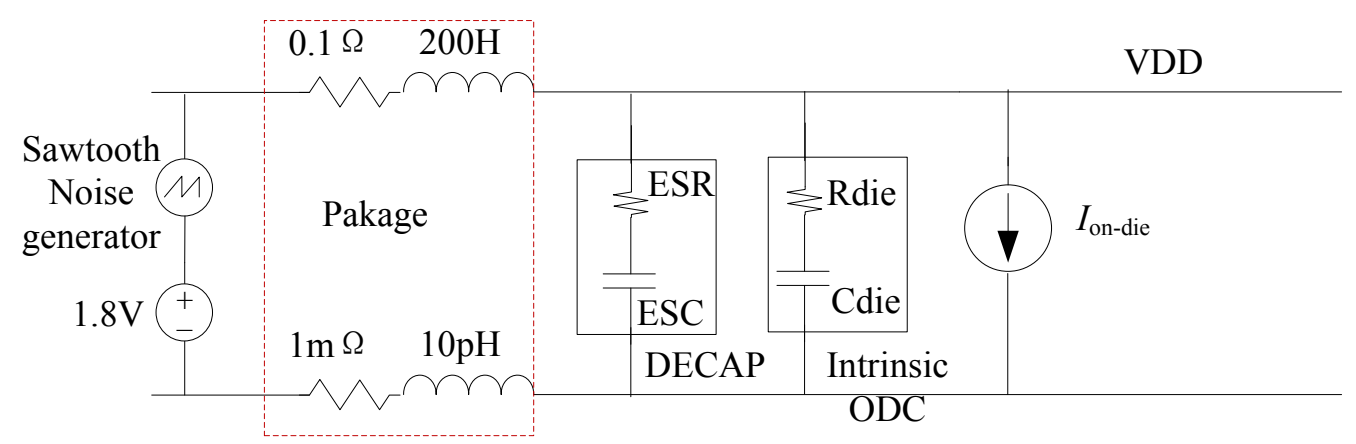

Figure 7.2: The testbench of sizing DECAP to satisfy the supply noise specification.

To size the DECAP, the Intrinsic ODC value should be extracted first, and then a time domain transient simulation is performed by sweeping the ESR and ESC combination and checking the power supply noise amplitude. The chosen ESC and ESR make the peak-to-peak supply noise amplitude equal to $10 \%$ of nominal supply voltage.

\subsubsection{Sizing the DECAP for the PLL based clock distribution circuit}

Traditionally the on-die decoupling capacitor is treated as a pure capacitor, so the Cdie-only 
of Intrinsic ODC is extracted with the method covered in Section 5.4.1. It is assumed that Intrinsic ODC is the same as that of Circuit-A. But with Cdie-only, a Cc extraction netlist of circuit has been used and the value of the extracted Cdie-only is $298.8 \mathrm{pF}$. With the Cdie-only value, the ESR and $\mathrm{ESC}$ of the DECAP are obtained as $\mathrm{ESR}=0.52 \Omega$ and $\mathrm{ESC}=690 \mathrm{pF}$. The supply noise amplitude has been decreased to $180 \mathrm{mVpp}$. The input supply voltage with $200 \mathrm{mVpp}$ amplitude sawtooth-shaped noise and the on-die supply voltage are shown in Figure 7.3.

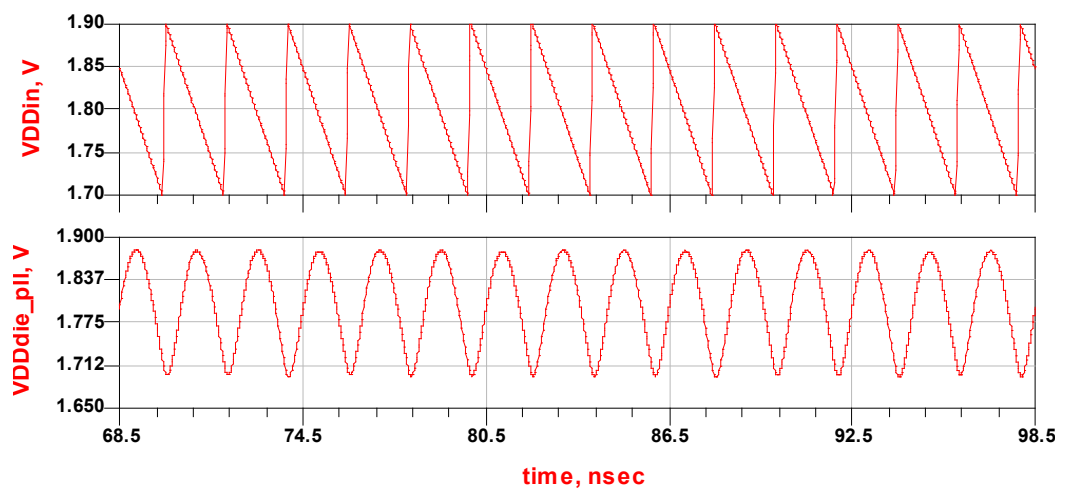

Figure 7.3: Input supply voltage VDDin and on-die supply voltage.

\subsubsection{Sizing the DECAP for the MDLL based clock distribution circuit}

The frequency domain Rdie and Cdie of the Intrinsic ODC are the same as those in Table 5.1. With the proposed method in Section 5.5, the approximate equivalent broadband circuit can be obtained and should be the same as that shown in Figure 5.23. With the Rdie/Cdie equivalent circuit and the testbench shown in Figure 7.2, the ESR and ESC for the DECAP 
are obtained as $\mathrm{ESR}=4.5 \Omega$ and the $\mathrm{ESC}=80 \mathrm{pF}$. The input supply voltage with a sawtooth-shaped noise and the on-die supply voltage reshaped by DECAP and Intrinsic ODC are shown in Figure 7.4.

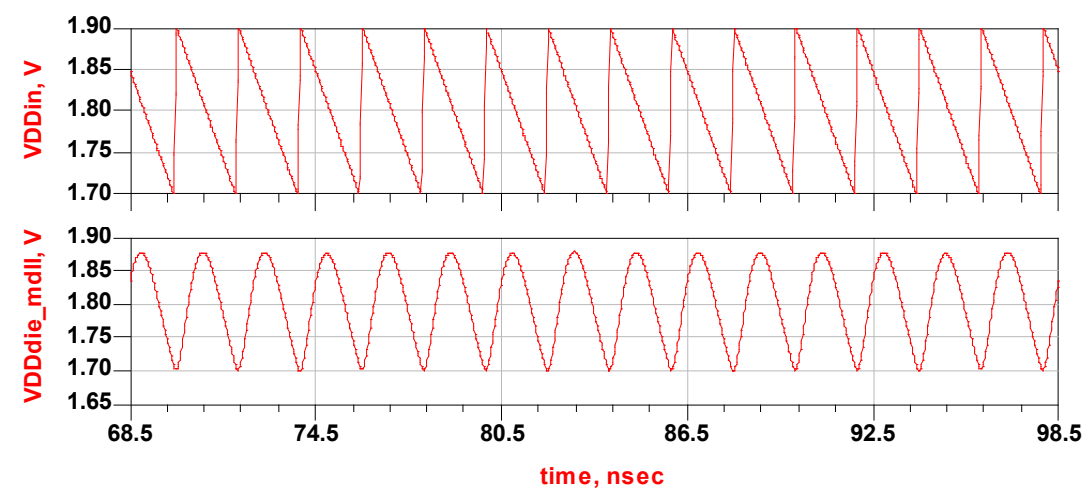

Figure 7.4: Input supply voltage VDDin and on-die supply voltage.

\subsubsection{Calculating the PSIJ}

The design specification in [84] requires that the jitter contributed from the clock distribution circuit should be smaller than 72ps (peak to peak) for a 2400Mbps DDR4 controller. The design specification of the peak-to-peak amplitude of the power supply noise is less than $\pm 5 \%$ of nominal supply voltage, which equals $180 \mathrm{mV}$ (peak-to-peak) in this design. The jitter contributed from the clock multiplier should be smaller than 40ps (peak to peak).

\subsubsection{Estimating the PSIJ with $\alpha$ factor method.}

The $\alpha$ factor for Buffer chain 4 is 0.9976 . The PSIJ is calculated with $\alpha$ factor method by 
equation (4.26),

$$
P S I J=\alpha \cdot \Delta V \cdot\left(t_{d} / V_{d d}\right)[p s]=0.9976 \times 180 m V_{p p}(1411 p s / 1.8 V)=140.8 p s
$$

The $140.76 \mathrm{ps}$ is much larger than the design specification of $72 \mathrm{ps}$. To satisfy the jitter specification with $\alpha$ factor method, one needs to either design a new buffer chain with a smaller $\alpha$ factor or lower the supply noise. Normally, a buffer chain is difficult to be improved since it is already optimized. To lower the supply noise, one can add more DECAPs or a voltage regulator, however, both methods will increase the cost.

\subsubsection{Estimating the PSIJ with PSIJS method}

To calculate the PSIJ, one can use the PSIJS expression of equation (4.22) proposed in Section 4.4.3. Because the PSIJS for Buffer chain 4 is obtained and shown in Figure 4.9(d), the power supply profile, VDDdie_mdll, is obtained and shown in Figure 7.4. The PSIJ is calculated through Matlab and the jitter sequence is plotted as shown in Figure 7.5. The peak-to-peak PSIJ is 55.11 ps which meets the design specification of 72 ps. So the buffer chain satisfies the design requirement and there is no need for further improvement. 


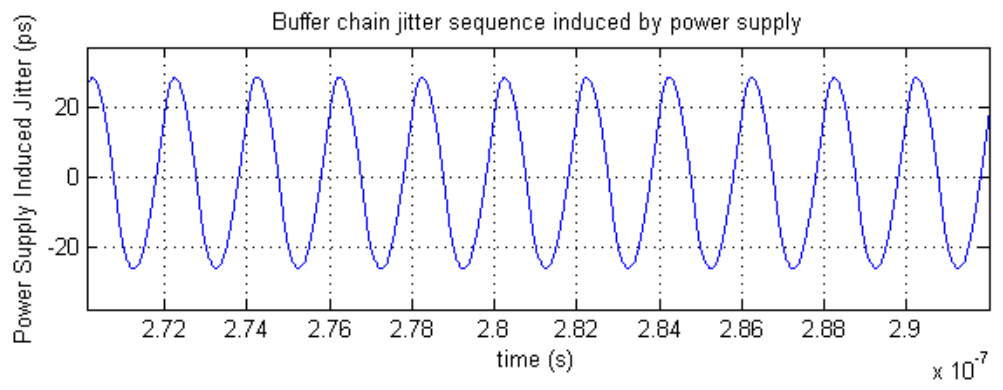

Figure 7.5: PSIJ sequences calculated through PSIJS of Buffer chain 4 calculated with equation (4.22).

\subsubsection{Performance Comparison between the Two Clock Distribution Circuits}

The Intrinsic ODC should be very similar no matter whether PLL or MDLL is used in Figure

7.1 because the physical sizes of PLL and the MDLL are much smaller than that of Circuit-A. Based on the measurement of Ride/Cdie of chipC in Chapter 6 and the correlation between simulation results in Section 5.6 and measurement results in Section 6.3.4, as a model of Intrinsic ODC, the approximate broadband equivalent circuit shown in Figure 5.23 should be much more accurate than the extracted Cdie-only capacitor. So the circuit in Figure 5.23 is used as the Intrinsic ODC in Figure 7.1 and with the DECAP sized in Section 7.1.1.1 and Section 7.1.1.2, the jitter sequences of the PLL based clock distribution circuit have been plotted in Figure 7.6 and the jitter sequences of the MDLL based clock distribution circuit have been plotted in Figure 7.7. 
The simulation results for the PLL based clock distribution circuit and the MDLL based one are summarized in Table 7.1. Based on the data from Table 7.1, one can see that both designs satisfy the design requirements. However, the clock distribution circuit with PLL has a problematic performance caused by over design. This is because the DECAP has been overestimated which is caused by the Intrinsic ODC model which is only a pure capacitor, the extracted Cdie-only capacitor. When comparing the two DECAPs respectively in the PLL-based and MDLL based circuit, one can see that the one sized with Cdie-only has $\mathrm{ESC} / \mathrm{ESR}=690 \mathrm{pF} / 0.52 \Omega$, the one sized with approximate broadband equivalent circuit has $\mathrm{ESC} / \mathrm{ESR}=80 \mathrm{pF} / 4.5 \Omega$, so the former has been overestimated 7.6 times in size.

The on-die supply voltage has been plotted in Figure 7.8 with the $\mathrm{ESC} / \mathrm{ESR}=690 \mathrm{pF} / 0.52 \Omega$ and with the equivalent broadband equivalent circuit as the model of Intrinsic ODC. It can be seen that peak-to-peak noise amplitude $=94 \mathrm{mVpp}$ which is $44 \%$ less than the design specification. With this noise profile, the jitter sequence of the Buffer chain has been obtained and plotted in Figure 7.9 by running a circuit simulation. It can be seen that the peak-to-peak jitter is $28.8 \mathrm{ps}$ (peak-to-peak) which is much smaller than the design specification of $72 \mathrm{ps}$. The jitter sequence of the Buffer chain has also been plotted in Figure 7.10 with the supply voltage profile, VDDdie_mdll, which is shown in Figure 7.4. The peak-to-peak jitter is $53.7 \mathrm{ps}$. It is very close to the value of $55.1 \mathrm{ps}$, which is calculated 
by using equation (4.22) in Section 7.1.2.2. This result further verifies the effectiveness of equation (4.22).

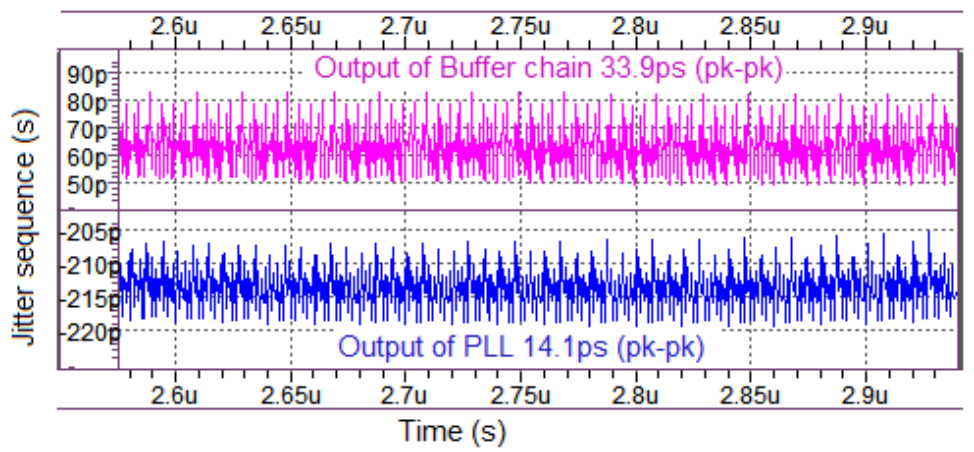

Figure 7.6: Jitter sequences plots at the output of Buffer chain and at the output of PLL.

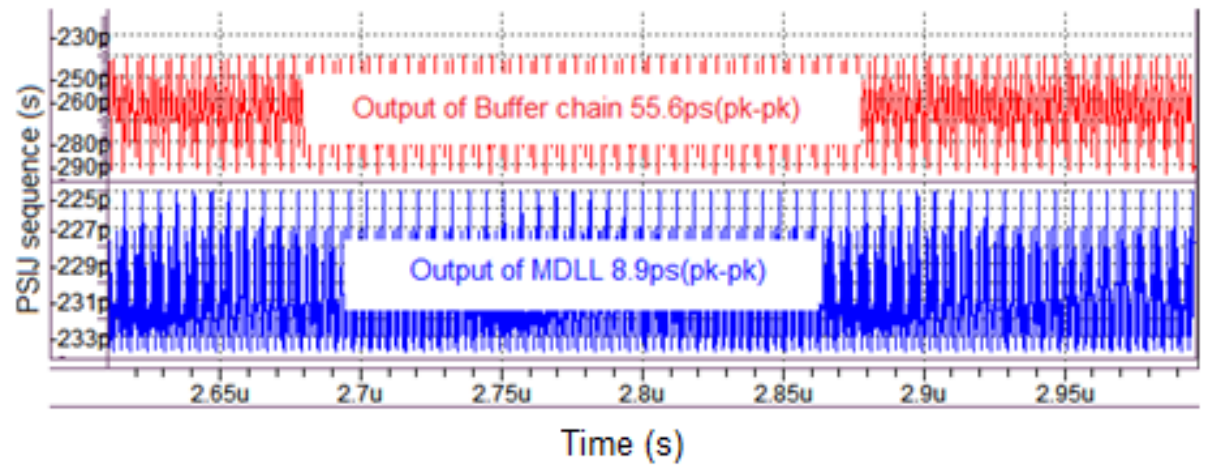

Figure 7.7: Jitter sequences plots at the output of Buffer chain and at the output of MDLL. 


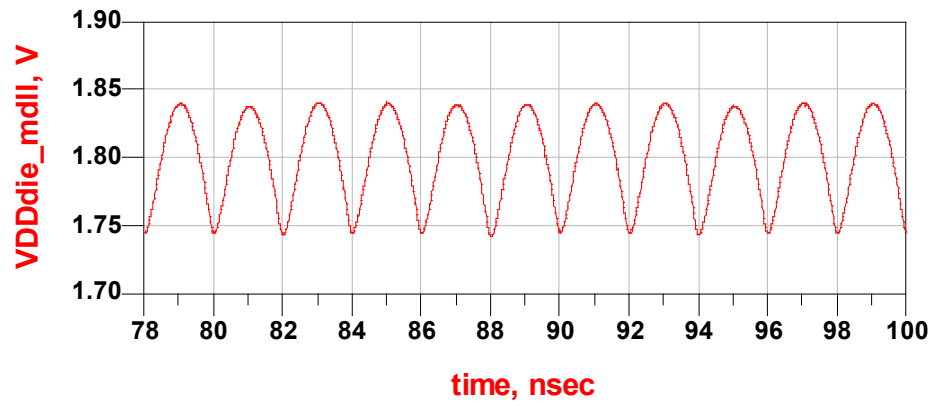

Figure 7.8: The on-die supply voltage with the $\mathrm{ESC} / \mathrm{ESR}=690 \mathrm{pF} / 0.52 \Omega$ and with the equivalent broadband equivalent circuit as the Intrinsic ODC model.

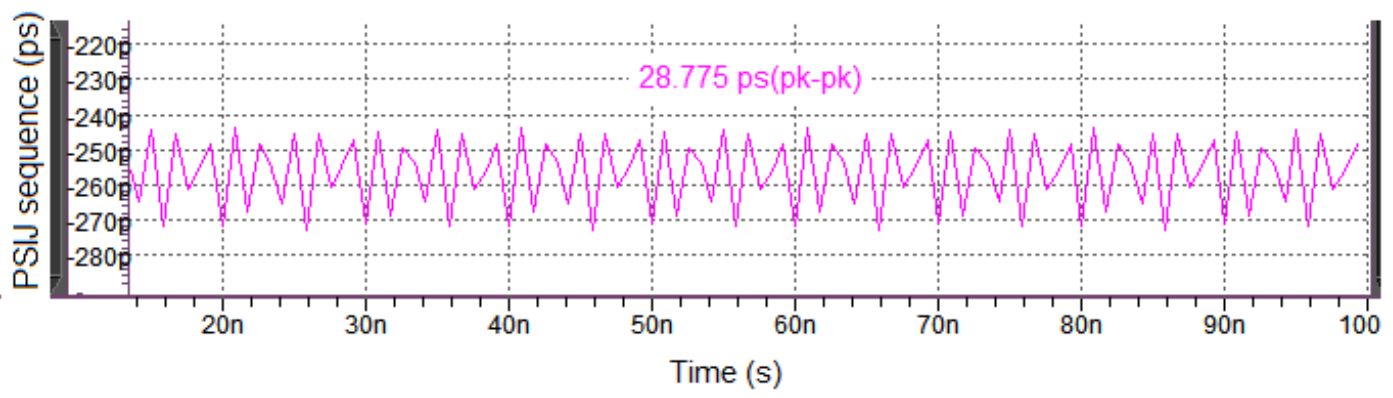

Figure 7.9: Buffer chain PSIJ sequence simulated with $\mathrm{ESC} / \mathrm{ESR}=690 \mathrm{pF} / 0.52 \Omega$ and the equivalent broadband equivalent circuit as the Intrinsic ODC model.

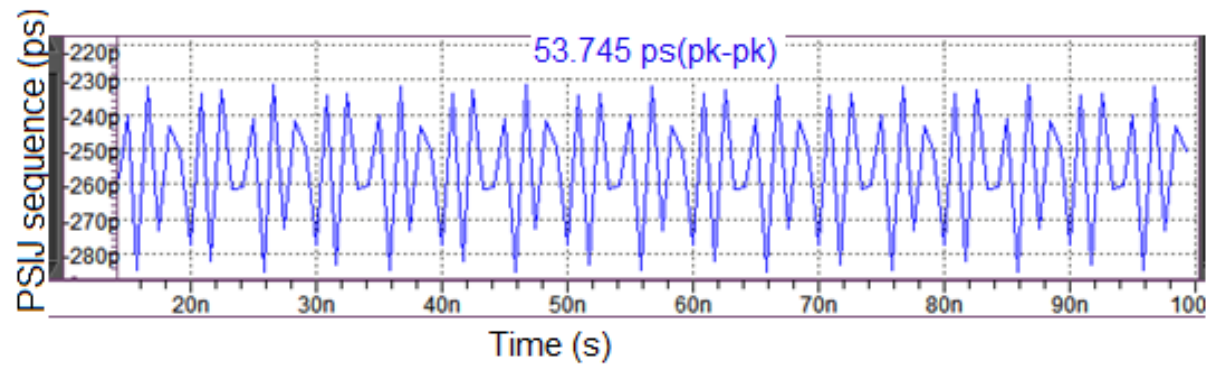

Figure 7.10: Buffer chain PSIJ sequence simulated with the supply voltage profile VDDdie_mdll as shown in Figure 7.4. 
Table 7.1 Summary of the simulation results from the clock distribution circuits with PLL/MDLL.

\begin{tabular}{|c|c|c|}
\hline & $\begin{array}{c}\text { PLL based clock } \\
\text { distribution circuit }\end{array}$ & $\begin{array}{c}\text { MDLL based clock } \\
\text { distribution circuit }\end{array}$ \\
\hline Intrinsic ODC model & Cdie-only & $\begin{array}{c}\text { Broadband equivalent } \\
\text { circuit }\end{array}$ \\
\hline DECAP & ESC/ESR $=690 \mathrm{pF} / 0.52 \Omega$ & $\mathrm{ESC} / \mathrm{ESR}=80 \mathrm{pF} / 4.5 \Omega$ \\
\hline $\begin{array}{c}\text { On-die supply noise } \\
\text { amplitude (Vpp) }\end{array}$ & $100 \mathrm{~m}$ & $180 \mathrm{~m}$ \\
\hline $\begin{array}{c}\text { Jitter measured at output of } \\
\text { Buffer chain ps (pk-pk) }\end{array}$ & 33.9 & 55.6 \\
\hline $\begin{array}{c}\text { Jitter measured at output of } \\
\text { clock multiplier ps (pk-pk) }\end{array}$ & 14.1 & 8.8 \\
\hline $\begin{array}{c}\text { Jitter simulated with Buffer } \\
\text { chain only ps (pk-pk) }\end{array}$ & 28.8 & 53.7 \\
\hline
\end{tabular}

From the item, Jitter measured at output of clock multiplier, in Table7.1, compared with PLL, MDLL has better jitter performance. With the proposed techniques in this dissertation, the clock distribution circuit for a DDR controller achieves a better optimization of tradeoff between performance and cost.

\subsection{Conclusion}

This dissertation focuses on the methods of improving power supply induced jitter with an application of DDR controllers. The application is vulnerable to power supply noise because the DDR interface takes single-ended signaling.

DLL-based clock multipliers are preferred since any jitter created by the on-chip noise is completely corrected when a clean reference clock edge arrives at the input of the DLL. In 
this dissertation, a reference-spur reduced MDLL is proposed and verified at both behavioral and the transistor level. The SPO between the reference edge and its counterpart of MDLL output is the dominant mechanism causing reference spurs in the spectrum of the MDLL output. With a high-gain stage inserted between phase detector/phase frequency detector and charge pump, the equivalent SPO has been decreased by a factor equal to the gain of the gain stage.

A large amount of buffer insertion in the memory interface clock path causes large power supply induced jitter. So it is necessary to use the PSIJS to quickly and accurately predict the jitter budget. However, an analytical or practical expression of PSIJS of buffer chain has not yet been available in published work. This dissertation presents a very simple and highly accurate expression of power supply-induced jitter sensitivity transfer function for a CMOS buffer chain. The transfer function is mainly a function of the maximum and minimum propagation delay of the buffer chain. The function can be easily obtained and used in jitter budget calculation.

To maintain low PSIJ, adding an on-die decoupling capacitor is an effective method. To prevent circuit overdesign and unnecessary on-die decoupling capacitance that results in larger silicon footprint, a method of converting the frequency domain equivalent Rdie and Cdie to an approximate broadband equivalent circuit is proposed, which can be readily used 
in time domain transient simulation. The proposed method, combined with a SPICE-like simulator which can extract frequency domain Rdie/Cdie, achieves an effective low-cost and highly accurate tool to extract Rdie/Cdie; this benefits designers without access to expensive commercial tools.

\subsection{Directions for Future Research}

Since power supply noise and induced jitter cover a wide research scope of both PDN and circuit design, this will continue to be a hot research area until CMOS technology stops scaling down. Considering this research, potential study in future could include:

1. The proposed reference spur reduced MDLL can be implemented.

2. The effectiveness of the proposed method, which converts the narrowband Rdie/Cdie to an approximate wideband equivalent circuit consisting of $\mathrm{N}$ series-RC subcircuits placed in parallel, has been verified in chapter 6 with practical measurements. However, the quiescent current is not modeled; in future the quiescent current can be modeled with a resistor in parallel with the proposed RC network (e.g. the $3 \Omega$ resistor in Figure 6.21). 


\subsection{Publications that Arose from the Research}

1. Wang, X.J. and Kwasniewski, T. (2015) A Static Phase Offset Reduction Technique for Multiplying Delay-Locked Loop. Circuits and Systems, 6, 13-19. http://dx.doi.org/10.4236/cs.2015.61002

2. Wang, X. J., and Kwasniewski, T. (2015) A reduced reference spur multiplying delay-locked loop. Int. J. Circ. Theor. Appl., doi: $\underline{10.1002 / c t a .2176}$

3. Xin Jie Wang and Tad Kwasniewski, "Propagation Delay Based Expression of Power Supply Induced Jitter Sensitivity for CMOS Buffer Chain”, IEEE Trans on Electromagnetic Compatibility, vol. 58, issue 2, pp. 627-630, January 2016

4. Xin Jie Wang, Leonard MacEachern and Tad Kwasniewski, "A Method of Obtaining an Approximate Broadband Equivalent Circuit for On-Die Power Grid Equivalent Resistance and Capacitance," (To be prepared for publication) 


\section{References}

[1] J. Monthie, V. Sreekumar, and R. Yashwante, "Impact of Power Supply Noise on Clock Jitter in High-Speed DDR Memory Interfaces," Proceedings of the $201326^{\text {th }}$ International Conference on VLSI Design: Held concurrently with the $12^{\text {th }}$ International Conference on Embedded Systems, pp. 262-266, January 2013.

[2] K. S. Oh and X. Yuan, High Speed Signaling: Jitter Modeling, Analysis, and Budgeting. Boston, MA: Pearson Education, 2012.

[3] R. Jakushokas, M. Popovich, A. V. Mezhiba, S. Kose, and E. G. Friedman, Power Distribution Networks With On-Chip Decoupling Capacitors, $2^{\text {nd }}$ ed.. New York: Springer, 2011.

[4] J. N. Tripathi, V. K. Sharma, H. Advani, P. N. Singh, H. Shrimali and R. Malik, "An Analysis of Power Supply Induced Jitter for a Voltage Mode Driver in High Speed Serial Links," 2016 IEEE $20^{\text {th }}$ Workshop on Signal and Power Integrity (SPI). Proceedings, pp. 4, 2016.

[5] E. Park, J. Kim, J. Lee and Y. Park, "Calculation of Power-supply-induced Jitter at a 3-D

IC Channel Including ESD Protection Circuits,” 2105 IEEE International Symposium on Electromagnetic Compatibility (EMC), Proceedings, pp. 1310-1314, 2015.

[6] D. Chan and M. R. Guthaus, "Analysis of Power Supply Induced Jitter in Actively 
De-skewed Multi-core Systems," Proceedings of the Eleventh International Symposium on Quality of Electronic Design, pp. 785-790, 2010.

[7] J. Sun, P. Wang and H. Zhang, "Reducing Power-supply and Ground Noise Induced Timing Jitter in Short Pulse Generation Circuits," 2015 IEEE Symposium on Electromagnetic Compatibility and Signal Integrity, pp. 17-21, 2015.

[8] J. Kim, J. Lee, E. Park and Y. Park, “An Enhanced Statistical Analysis Method for I/O Links Considering Supply Voltage Fluctuations and Intersymbol Interference," IEEE Transactions on Components, Packaging and Manufacturing Technology, vol. 5, no. 8, pp. 1129-1141, August 2015.

[9] D. Oh, Y. Shim and G. Chen, "Supply Noise Induced Jitter Modeling and Optimization for High-speed Interfaces,” 2015 IEEE Custom Integrated Circuits Conference (CICC), Proceedings, pp. 42, September 2015.

[10] Y. Shim, D. Oh, T. Hoang and Y. Ke, “A Jitter Equalization Technique for Minimizing Supply Noise Induced Jitter in High Speed Serial Links," 2014 IEEE International Symposium on Electromagnetic Compatibility (EMC), pp. 827-832, August 2014.

[11] Microsemi Corporation (2012 September). Simultaneous Switching Noise and Signal Integrity [Online]. Available:

http://www.microsemi.com/document-portal/doc_view/130042-ac263-simultaneous-switchin 


\section{g-noise-and-signal-integrity-app-note}

[12] D. J. Foley and M. P. Flynn, "CMOS DLL-based 2-V 3.2-ps Jitter 1-GHz Clock Synthesizer and Temperature-compensated Tunable Oscillator," IEEE Journal of Solid-State Circuits, vol. 36, no. 3, pp. 417-423, Mar. 2001.

[13] Q. J. Du, J. C. Zhuang, and T. Kwasniewski, “A Low-phase Noise, Anti-harmonic Programmable DLL Frequency Multiplier with Period Error Compensation for Spur Reduction," IEEE Trans. Circuits Syst. II 2006, vol. 53, no. 11, pp. 1205-1209, November 2006.

[14] T. A. Ali, A. Hafez, R. Drost, R. Ho and C.-K. K. Yang, "A 4.6GHz MDLL with -46dBc

Reference Spur and Aperture Position Tuning," 2011 IEEE International Solid-State Circuits Conference (ISSCC 2011), pp. 466-468, February 2011.

[15] B. M. M. Helal, Z. Straayer, G.-Y. Wei and M. H. Perrott, "A Highly Digital MDLL-based Frequency Multiplier that Leverages a Self-scrambling Time-to-digital Converter to Achieve Subpicosecond Jitter Performance," IEEE Journal of Solid-State Circuits 2008, vol. 43, no. 4, pp. 855-863, April 2008.

[16] G. Marzin, S. Levantino, C. Samori and A. L. Lacaita , “A 1.7GHz MDLL-Based Fractional-N Frequency Synthesizer with 1.4ps RMS Integrated Jitter and 3mW Power Using a 1b TDC," 2014 IEEE International Solid-State Circuits Conference Digest of 
Technical Papers (ISSCC), pp. 360-361, February 2014.

[17] S. Kundu, B. Kim, and C.H. Kim, "A 0.2-to-1.45GHz Subsampling Fractional-N All-digital MDLL with Zero-offset Aperture PD-based Spur Cancellation and In-situ timing Mismatch Detection," 2016 IEEE International Solid-State Circuits Conference (ISSCC), pp. 326-327, 2016.

[18] M.-J.E. Lee, W.J. Dally, T. Greer, Hiok-Tiaq Ng, Farjad-Rad, R.,Poulton, J., Senthinathan, R., "Jitter Transfer Characteristics of Delay-locked Loops - Theories and Design Techniques", IEEE Journal of Solid-State Circuits, vol. 38, no. 4, pp. 614 - 621, April 2003 .

[19] X. J. Wang and T. Kwasniewski. "A Static Phase Offset Reduction Technique for Multiplying Delay-Locked Loop," Circuits and Systems, vol. 6, pp. 13-19, 2015.

[20] Wang, X. J., and Kwasniewski, T., "A Reduced Reference Spur Multiplying Delay-locked Loop," International Journal of Circuit Theory and Applications, vol. 44, no. 8, pp. 1620-1627, Aug. 2016.

[21] Y.-F. Liu, B. Wang, M. Xu, X. Liu, J. Z. Chen, and M. Desmith, "Correlation of On-Die Capacitance for Power Delivery Network", 2008 IEEE $17^{\text {th }}$ Conference on Electrical Performance of Electronic Packaging (EPEP), pp. 123-126, 2008.

[22] F. Herzel and B. Razavi, "A Study of Oscillator Jitter due to Supply and Substrate 
Noise," IEEE Transactions on Circuits and Systems II: Analog and Digital Signal Processing, vol. 46, no. 1, pp. 56-62, January 1999.

[23] R. Schmitt, L. Hai, C. Madden, and C. Yuan, "Investigating the Impact of Supply Noise on the Jitter in Gigabit I/O Interfaces," IEEE $16^{\text {th }}$ Topical Meeting on Electrical Performance of Electronic Packaging, pp. 189-192, October 2007.

[24] Y. Shim, D. Oh, "System Level Modeling of Timing Margin Loss Due to Dynamic Supply Noise for High-Speed Clock Forwarding Interface." IEEE Transactions on Electromagnetic Compatibility. 2016 Aug;58(4): pp.1349-1358.

[25] W. T. Beyene, A. Amirkhany, C. Madden, H. Lan, L. Yang, K. Kaviani, S. Mukherjee, D. Secker and R. Schmitt, "Design and analysis of $12.8 \mathrm{~Gb} / \mathrm{s}$ single-ended signaling for memory interface." In2011 IEEE 20th Conference on Electrical Performance of Electronic Packaging and Systems, 2011 Oct 23, pp. 135-138.

[26] C. Hwang, J. Kim, B. Achkir, and J. Fan, “Analytical Transfer Functions Relating Power and Ground Voltage Fluctuations to Jitter at a Single-ended Full-swing Buffer," IEEE Transactions on Components, Packaging and Manufacturing Technology, vol. 3, no. 1, pp. 113-125, January 2013.

[27] J. Kim, S. De, K. Shringarpure, S. Pan, B. Achkir, J. Fan and J. L. Drewniak, "Analytical Expressions for Transfer Function of Supply Voltage Fluctuation to Jitter at a 
Single-ended Buffer," in 2011 IEEE International Symposium on Electromagnetic Compatibility - EMC 201, pp. 422-427, August 2011.

[28] E. Park, H. Kim, K. Shon, and J. Kim, "Analytical Jitter Estimation of Two-stage Output Buffers with Supply Voltage Fluctuations," 2014 IEEE International Symposium on Electromagnetic Compatibility (EMC), pp. 69-74, August 2014.

[29] E. Park, H. Kim, J. Shim, Y.-J. Kim, Y.-S. Kim and J. Kim, “Analytical Calculation of Jitter Probability Density at Multistage Output Buffers Due to Supply Voltage Fluctuations," IEEE Transactions on Electromagnetic Compatibility, vol. 57, no. 4, pp. 796 - 806, Aug. 2015.

[30] X. J. Wang and T. Kwasniewski, "Propagation Delay Based Expression of Power Supply Induced Jitter Sensitivity for CMOS Buffer Chain”, IEEE Transactions on Electromagnetic Compatibility, vol. 58, no. 2, pp. 627-630, April 2016.

[31] X. Liu and Y.-F. Liu, "The Extraction and Measurement of On-die Impedance for Power Delivery Analysis," 2009 IEEE $18^{\text {th }}$ Conference on Electrical Performance of Electronic Packaging and Systems, pp. 195-198, 2009.

[32] M. Shin, C. Yoon, J. Cho, J. Shim, Y. Shim and J. Kim, “Analysis of DLL Jitter Affected by Power Supply Noise on Power Distribution Network," International Symposium on Electromagnetic Compatibility, pp. 421-424, July 2009. 
[33] D. H. Baek, B. Kim, H.-J. Park and J.-Y. Sim, "A 5.67mW 9Gb/s DLL-based Reference-less CDR with Pattern-dependent Clock-embedded Signaling for Intra-panel Interface," 2014 IEEE International Solid-State Circuits Conference Digest of Technical Papers (ISSCC), pp. 48-49, February 2014.

[34] K. Ryu, J. Jung, D-H. Jung, J. H. Kim and S.-O. Jung, "High-Speed, Low-Power, and Highly Reliable Frequency Multiplier for DLL-Based Clock Generator,' IEEE Transactions on Very Large Scale Integration (VLSI) Systems, vol. 24, no. 4, pp. 1484 - 1492, April 2016.

[35] Y.-H. Tu, H.-H. Chang, C.-L. Hung and K.-H. Cheng, "A 3 GHz DLL-based Clock Generator with Stuck Locking Protection," IEEE International Conference on Electronics, Circuits, and Systems (ICECS), pp. 106-109, 2010.

[36] R.-M. Weng, C.-Y. Liu, M.-H. Liang and Y.-F. Kuo, "A 19 2MHz to $1.946 \mathrm{GHz}$ Programmable DLL-Based Frequency Multiplier for RF Applications," 2007 Digest of Technical Papers. International Conference on Consumer Electronics, pp. 1-2, January 2007.

[37] M. Kim, S. Choi, T. Seong and J. Choi, "A Low-jitter and Fractional-resolution Injection-locked Clock Multiplier Using a DLL-based Real-time PVT Calibrator with Replica-delay Cells," IEEE Journal of Solid-State Circuits, vol. 51, no. 2, pp. 401-411, February 2016.

[38] B. Kim, T. Weigandt, and P. Gray, "PLL/DLL System Noise Analysis for Low Jitter 
Clock Synthesizer Design," 1994 IEEE International Symposium on Circuits and Systems, vol. 4, pp. 31-34, May 1994.

[39] T. Weigandt, B. Kim, and P. Gray, "Analysis of Timing Jitter in CMOS ring Oscillators," 1994 IEEE International Symposium on Circuits and Systems, vol. 4, pp. 27-30, June 1994.

[40] C. Kim, I. C. Hwang, and S.-M. Kang, “A Low-power Small-area \pm 7.28-ps-jitter 1-GHz DLL-based Clock Generator," IEEE Journal of Solid-StateCircuits, vol. 37, no. 11, pp. 1414-1420, November 2002.

[41] S. Revathi and R.Radhika, "Signal Integrity Modeling for High-speed DDRx Using Chip Package Board analysis," International Journal of Power Control Signal and Computation (IJPCSC), vol.3, no.1, pp. 105-109, January - March 2012.

[42] A. Elshazly, R. Inti, B. Young and P. K. Hanumolu, "Clock Multiplication Techniques Using Digital Multiplying Delay-Locked Loops," IEEE Journal of Solid-State Circuits 2013, Vol. 48, No. 6, pp. 1416-1428, June 2013.

[43] R. Farjad-Rad and et al., "A Low-power Multiplying DLL for low-jitter multigigahertz Clock Generation in Highly-integrated Digital Chips," IEEE Journal of Solid-State Circuits, vol. 37, no. 12, pp. 1804-1812, December 2002.

[44] Q. J. Du, "A CDR with a Digital Threshold Decision Technique and a Cyclic Reference 
Injected DLL Frequency Multiplier with a Period Error Compensation Loop," Ph.D. dissertation, Carleton University, December 2007.

[45] G. Chien and P. R. Gray, "900-MHz Local Oscillator Using a DLL-based Frequency Multiplier Technique for PCS Applications," IEEE Journal of Solid-State Circuits, vol. 35, no.12, pp. 1996 -1999, December 2000.

[46] H. Huung and J. Shen, "A DLL-Based Programmable Clock Generator Using Threshold-Trigger Delay Element and Circular Edge Combiner," IEEE Asia-Pacific Conference on Advanced System Integrated Circuits (APASIC2004), pp. 76-79, August 2004.

[47] J.-H. Kim, Y.-H. Kwak, M. Kim, S.-W. Kim, C. Kim, “A 120-MHz-1.8-GHz MOS DLL-based Clock Generator for Dynamic Frequency Scaling," IEEE Journal of Solid-State Circuits, vol. 41, no. 9, pp. 2077-2082, September 2006.

[48] C.-C. Wang, H.-C. She and R. Hu, "A 1.2 GHz Programmable DLL-based Frequency Multiplier for Wireless Application," IEEE Transaction on VLSI Systems, vol. 12, no. 12, pp. 1377-1381, 2004.

[49] G.-Y. Wei, J.T. Stonick, D. Weinlader and et al., “A 500MHz MP/DLL Clock Generator for $5 \mathrm{~Gb} / \mathrm{s}$ Backplane Transceiver in $0.25 \mu \mathrm{m}$ CMOS," 2003 IEEE International Solid-State Circuits Conference. Digest of Technical Papers (Cat. No.03CH37414), vol.1, pp. 464-465, 
February 2003.

[50] S. Ye, L. Jansson and I. Galton, “Techniques for In-band Phase Noise Suppression in Re-Circulating DLLs", Proceedings of the IEEE 2003 Custom Integrated Circuits Conference, pp. 297-300, September 2003.

[51] Synopsys (2016). Design Ware DDR3/2 SDRAM PHY IP [Online]. Available: https://www.synopsys.com/dw/ipdir.php?ds=dwc ddr3 phy

[52] K. Shu and E. Sanchez-Sinencio, CMOS PLL Synthesizers: Analysis and Design. New York: Springer, 2005.

[53] W. Rhee, "Design of High Performance CMOS Charge Pumps in Phase Locked Loop". Proceedings of the 1999 IEEE International Symposium on Circuits and Systems VLSI, vol. 1, pp. 545-548, June 1999.

[54] D. Fischette. (2009). First Time, Every Time Practical Tips for Phase Locked Loop Design [Online]. Available:http://www.delroy.com/PLL_dir/tutorial/PLL_tutorial_slides. [55] B. Razavi, Design of Analog CMOS Integrated Circuits. New York: McGraw-Hill, 2001.

[56] D. A. Johns and K. Martin, Analog Integrated Circuit Design. New York: Wiley, 1997.

[57] X. Gao, E. Klumperink, G. Socci, M. Bohsali, and B. Nauta, "Spur reduction techniques for phase-locked loops exploiting a sub-sampling phase detector," IEEE J. Solid-State 
Circuits, vol. 45, no. 9, pp. 1809-1821, Sep. 2010.

[58] X. Gao, E. Klumperink, M. Bohsali, and B. Nauta, "A low-noise sub-sampling PLL in which divider noise is eliminated and PD/CP noise is not multiplied by $\mathrm{N}^{2}$," IEEE J. Solid-State Circuits, vol. 44, no. 12, pp. 3253-3263, Dec. 2009.

[59] Meng-Lieh Sheu, Ta-Wei Lin, Wei-Hung Hsu, "Wide Frequency Range Voltage Controlled Ring Oscillator based on Transmission Gates," IEEE International Symposium on Circuits and Systems (ISCAS), vol. 3, pp. 2731-2734, May 2005.

[60] In-Chul Hwang, Chulwoo Kim, Sung-Mo Kang, "A CMOS self-regulating VCO with low supply sensitivity”, IEEE J. Solid-State Circuits, vol. 39, pp.42-48, January 2004.

[61] N. Retdian, S. Takagi, and N. Fujii, "Voltage controlled ring oscillator with wide tuning range and fast voltage swing," in Proc. IEEE AsiaPacific Conf. Advanced Syst. Integr. Circuits, pp. 201-204, Aug. 2002.

[62] X. Wang and A. Martin, "On-die Supply-induced Jitter Behavioral Modeling”, 2013 IEEE $22^{\text {nd }}$ Conference on Electrical Performance of Electronic Packaging and Systems (EPEPS 2013), pp. 147-150, 2013.

[63] R. Schmitt, L. Hai, C. Madden, and C. Yuan, “Analysis of Supply Noise Induced Jitter in Gigabit I/O Interfaces," International Engineering Consortium - DesignCon 2007, vol. 3, pp. 1716-1738, January 2007. 
[64] D. Ho and Y. Shim, "A Simple and Innovative Circuit Technique to Tackle Power Supply Induced Jitter in High Speed Serial Links for 25Gbps and beyond,” DesignCon 2015, January, 2015.

[65] L. H. Chen, M. M. Sadowska, and F. Brewer, "Buffer Delay Change in the Presence of Power and Ground Noise," IEEE Transactions on Very Large Scale Integration (VLSI) Systems, vol. 11, no. 3, pp. 461-473, June 2003.

[66] E. Park, H. Kim, J. Shim, Y. Kim, Y. Kim, J. Kim, “Analytical Calculation of Jitter Probability Density at Multistage Output Buffers due to Supply Voltage Fluctuations," IEEE Transactions on Electromagnetic Compatibility, vol. 57, no.4, pp.796-806, August 2015.

[67] Y. Shim, J. Lee J. and Kim, "Fast, Precise and Broadband Modelling of Induced Clock Jitter by SSN Coupling," DesignCon 2012: Where Chipheads Connect, vol. 3, pp. 1631-1675, January 2012.

[68] H. Neil, E. Weste, and D. M. Harris, CMOS VLSI Design: A Circuits and Systems Perspective, Boston: Pearson/Addison-Wesley, $4^{\text {th }}$ Edition, 2010.

[69] M. Swaminathan and A. E. Engin, Power Integrity Modeling and Design for Semiconductor and Systems, Prentice Hall, 2007.

[70] L. Smith, S. Sun, M. Sarmiento, Z. Li, and K. Chandrasekar, "On-Die Capacitance Measurements in the Frequency and Time Domains," DesignCON 2011, vol.2, pp. 
1249-1273, 2011.

[71] I. Novak, "Frequency-Domain Power-Distribution Measurements - An Overview," HP-TF1. TecForum, DesignCon East, June 23, 2003, Boston, MA.

[72] E. Bogatin, Signal and Power Integrity Simplified, $2^{\text {nd }}$ ed.. Upper Saddle River, N.J: Prentice Hall, 2010.

[73] C. Q. Li, B. E. Cheah, W. L. Lee and W. C. Sam, "Intrinsic Capacitance Extraction and Estimation for System-On-Chip Power Delivery Development," 2012 IEEE Asia Pacific Conference on Circuits and Systems (APCCAS 2012), pp. 388-391, December 2012.

[74] E. Bogatin, "ESPI: Essential Principles of Signal Integrity - Build your Engineering Intuition to Solve SI Problems," Bogatin Enterprises 2013.

[75] L. Scheffer, L. Lavagno and G. Martin, EDA for IC Implementation, Circuit Design, and Process Technology. Boca Raton, FL: CRC Taylor \& Francis, 2006.

[76] Calibre ${ }^{\circledR}$ xRC TM User’s Manual, Software Version 2009.1.

[77] L. D. Smith, R. Anderson, T. Roy, "Chip-Package Resonance in Core Power Supply Structures for a High Power Microprocessor," Advances in Electronic Packaging, vol. 1, pp. 63-68, July 2001.

[78] I. Novak (2000). Measuring Milliohms and PicoHenrys in Power Distribution Networks [Online]. Available: 
http://electrical-integrity.com/Paper_download_files/DC00_MeasuringMiliohms_slides.pdf.

[79] F. Guo and S. Catherine, "IO Power Delivery Network Impedance Measurement at Chip Level," 2014 IEEE $23^{\text {rd }}$ Conference on Electrical Performance of Electronic Packaging and Systems (EPEPS). Proceedings, pp. 109-112, October 2014.

[80] W.-W. Wong, S.-M. Low and B. Beker, "Low Impedance Characterization of Power

Delivery Network on Substrate Level for High Speed Digital Applications," 2013 IEEE $15^{\text {th }}$ Electronics Packaging Technology Conference (EPTC 2013), pp. 726-730, December 2013.

[81] Agilent Technologies, Advanced Calibration Techniques for Vector Network Analyzers, Agilent Technologies, Inc. 2006.

[82] Mantra VLSI. (2014, October 14). Flip-chip and wire bonding [Online]. Available: http://mantravlsi.blogspot.ca/2014/10/flip-chip-and-wire-bonding.html

[83] Preeti S., Chauhan, Anupam Choubey, ZhaoWei Zhong and Michael G. Pecht, Copper Wire Bonding, Springer 2014.

[84] Synopsys (2013). Guidelines for Implementing Signaling Environments for DDRn Interfaces: PCB, Package, Power, and Timing Budgets. DWC App Note, Revision 4.1, June 14,2013

[85] C. Hwang, T.-L. Chu, and W.-C. Chen, "A Clock Generator Based on Multiplying Delay-locked Loop," 2014 27 th IEEE International System-on-Chip Conference (ISOCC), 
pp. 98-102, 2014.

[86] C.-K. K. Yang, "Delay-locked loops-An overview," in Phase-Locking in High Performance Systems, B. Razavi, Ed. New York: IEEE Press, March 2003. 